UNITED STATES

DEPARTMENT OF THE INTERIOR

GEOLOGICAL SURVEY

COMPILATION OF WELL AND GROUND-WATER QUALITY DATA, GROVELAND-COLLINS AND SURROUNDING AREAS NEAR BLACKFOOT, BINGHAM COUNTY, IDAHO

By D. J. Parliman

Open-File Report 86-127

Prepared in cooperation with the

IDAHO DEPARTMENT OF HEALTH AND WELFARE

Boise, Idaho 
UNITED STATES DEPARTMENT OF THE INTERIOR

DONALD PAUL HODEL, Secretary

GEOLOGICAL SURVEY

Dallas L. Peck, Director

For additional information write to:

Idaho Office Chief U.S. Geological Survey, WRD 230 Collins Road Boise, ID 83702 (208) 334-1750
Copies of this report can be purchased from:

U.S. Geological Survey Western Distribution Branch Open-File Services Section Box 25425, Federal Center Denver, CO 80225 (303) 236-7476 


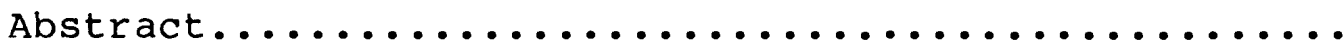

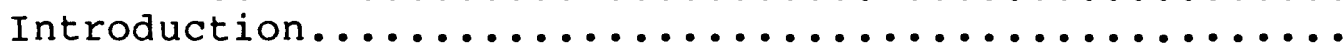

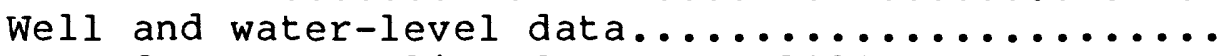

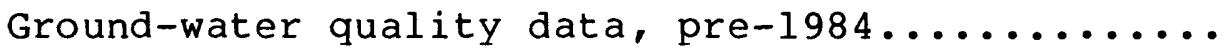

Ground-water quality data, 1984 and $1985 \ldots \ldots \ldots \ldots$

References cited.

\section{ILLUSTRATIONS}

Figure 1. Diagram showing well-location system...... ii

2. Map showing location of Groveland-Collins

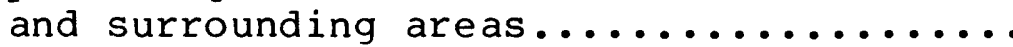

3. Map showing locations of wells selected for use during 1984-85 study.

4. Map showing locations of wells with selected pre-1984 and 1984-85 waterquality data.

TABLES

Table 1. Well and water-level data............... 8

2. Ground-water quality data, pre-1984........ 51

3. Ground-water quality data, 1984 and $1985 \ldots . .86$

4. Selected trace-element data, $1984 \ldots . . . . . .112$

5. Statistical summary of selected water-

quality data, 1984 and $1985 \ldots \ldots \ldots \ldots \ldots . . . .113$ 


\section{CONVERSION FACTORS}

\section{Multiply}

acre

foot ( $f t)$

micromho per

centimeter at $25^{\circ} \mathrm{C}$

( $\mu \mathrm{mho} / \mathrm{cm}$ )

mile (mi)
$\underline{B y}$

4,047

0.3048

1.000

1.609
To obtain

square meter

meter

microsiemens per

centimeter at

$25^{\circ} \mathrm{C}$

kilometer

Temperature in ${ }^{\circ} \mathrm{C}$ (degrees Celsius) can be converted to

${ }^{\circ} \mathrm{F}$ (degrees Fahrenheit) as follows:

$$
{ }^{\circ} \mathrm{F}=(1.8)\left({ }^{\circ} \mathrm{C}\right)+32
$$

All water temperatures are reported to the nearest $0.5^{\circ} \mathrm{C}$.

\section{WELL-LOCATION SYSTEM}

The well-location system (fig. 1) indicates the position of wells within the official rectangular subdivision of public lands, with reference to the Boise base line and meridian. The first two segments of the number designate township (north or south) and range (east or west). The third segment gives the section number; three letters, which indicate $\frac{1}{4}$ section (160-acre tract), $\frac{1}{4}-\frac{1}{4}$ section (40-acre tract), and $\frac{1}{4}-\frac{1}{4}-\frac{1}{4}$ section (10-acre tract); and the serial number of the well within the tract. In areas where well locations are tightly clustered, a fourth letter is added to indicate $\frac{1}{4}-\frac{1}{4}-\frac{1}{4}-\frac{1}{4}$ section (2.5-acre tract).

The U.S. Geological Survey in Idaho indicates quarter sections by the letters $A, B, C$, and D in counterclockwise order from the northeast quarter of each section. Within quarter sections, 40-acre, 10-acre, and 2.5-acre tracts are lettered in the same manner. For example, well 3S-35E$6 \mathrm{CDAl}$ is the first well inventoried in the $\mathrm{NE}^{\frac{1}{4}}$ of the $\mathrm{SE} \frac{1}{4}$ of the SW $\frac{1}{4}$, section 6 , township 3 south, range 35 east. 


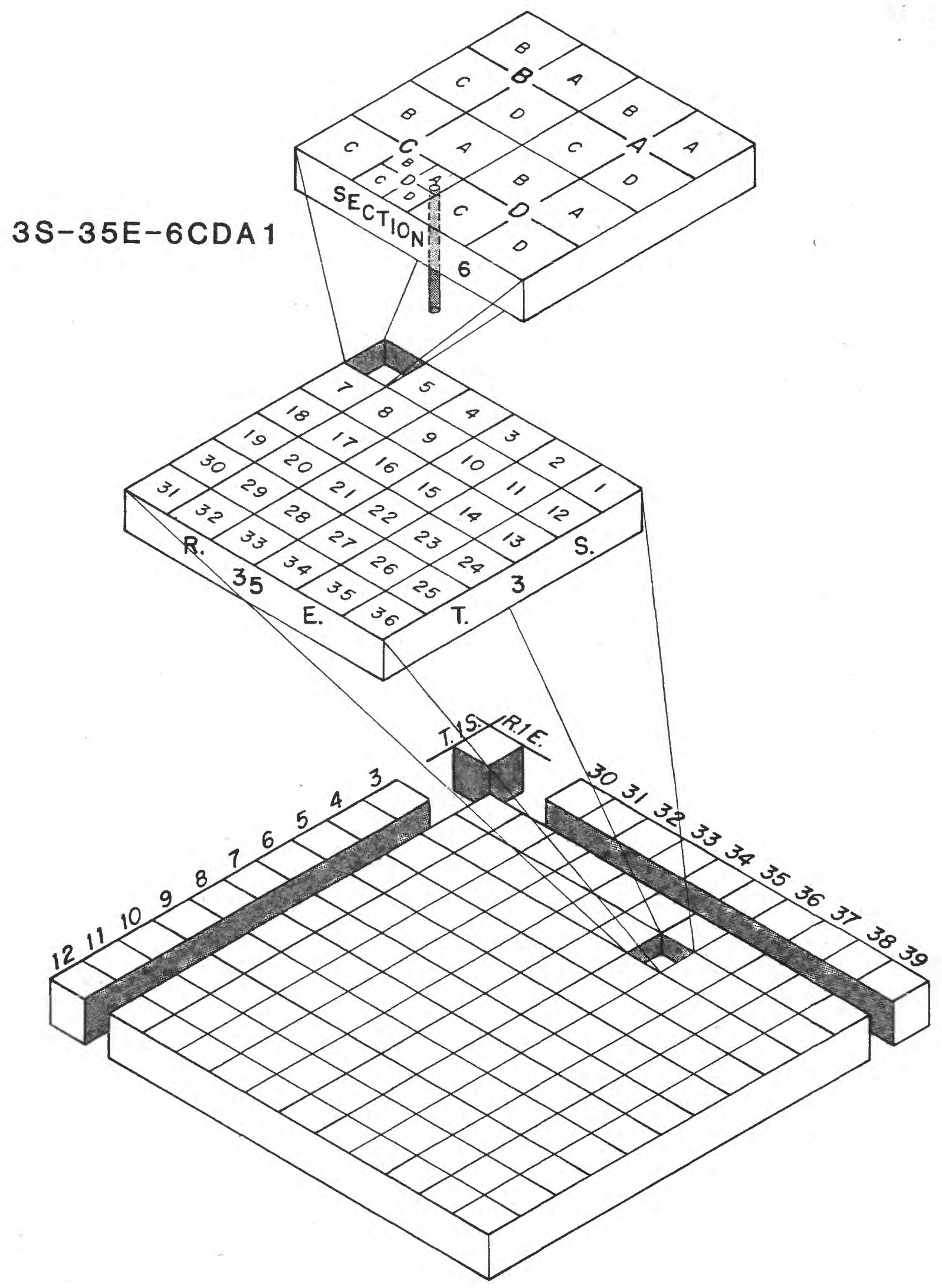

Figure 1.--Well location system. 


\title{
COMPILATION OF WELL AND GROUND-WATER QUALITY DATA, GROVELAND-COLLINS AND SURROUNDING AREAS NEAR BLACKFOOT, BINGHAM COUNTY, IDAHO
}

By

D. J. Parliman

\begin{abstract}
Well-construction, geologic, and water-level data from 1978 to 1985 were compiled for 163 sites near Blackfoot, Idaho. Ground-water quality data were compiled for 51 sites for the period 1961-83 and for 54 sites for the period 1984-85. Data were collected in support of the hydrologic and water-quality components of the U.S. Geological Survey's ground-water contamination investigation near Blackfoot.
\end{abstract}

\section{INTRODUCTION}

The purpose of this report is to present wel1construction, geologic, water-level, and water-quality data compiled to determine ground-water hydrology and water chemistry in the Groveland-Collins and surrounding areas near Blackfoot, Idaho (fig. 2). These data were compiled as part of the hydrologic and water-quality components of a ground-water contamination investigation by the U.S. Geological survey, conducted in cooperation with the Idaho Department of Health and Welfare, Division of Environment. A companion interpretive study is currently in progress (D. J. Parliman, U.S. Geological Survey, unpublished material, 1986).

Water-level and water-quality data in this report were collected by personnel from the Idaho Department of Water Resources; Bingham County; Idaho Department of Health and Welfare, Southeastern District Health Department; Idaho Department of Health and Welfare, Division of Environment; and U.S. Geological Survey. Sources of data are explained in following sections. 


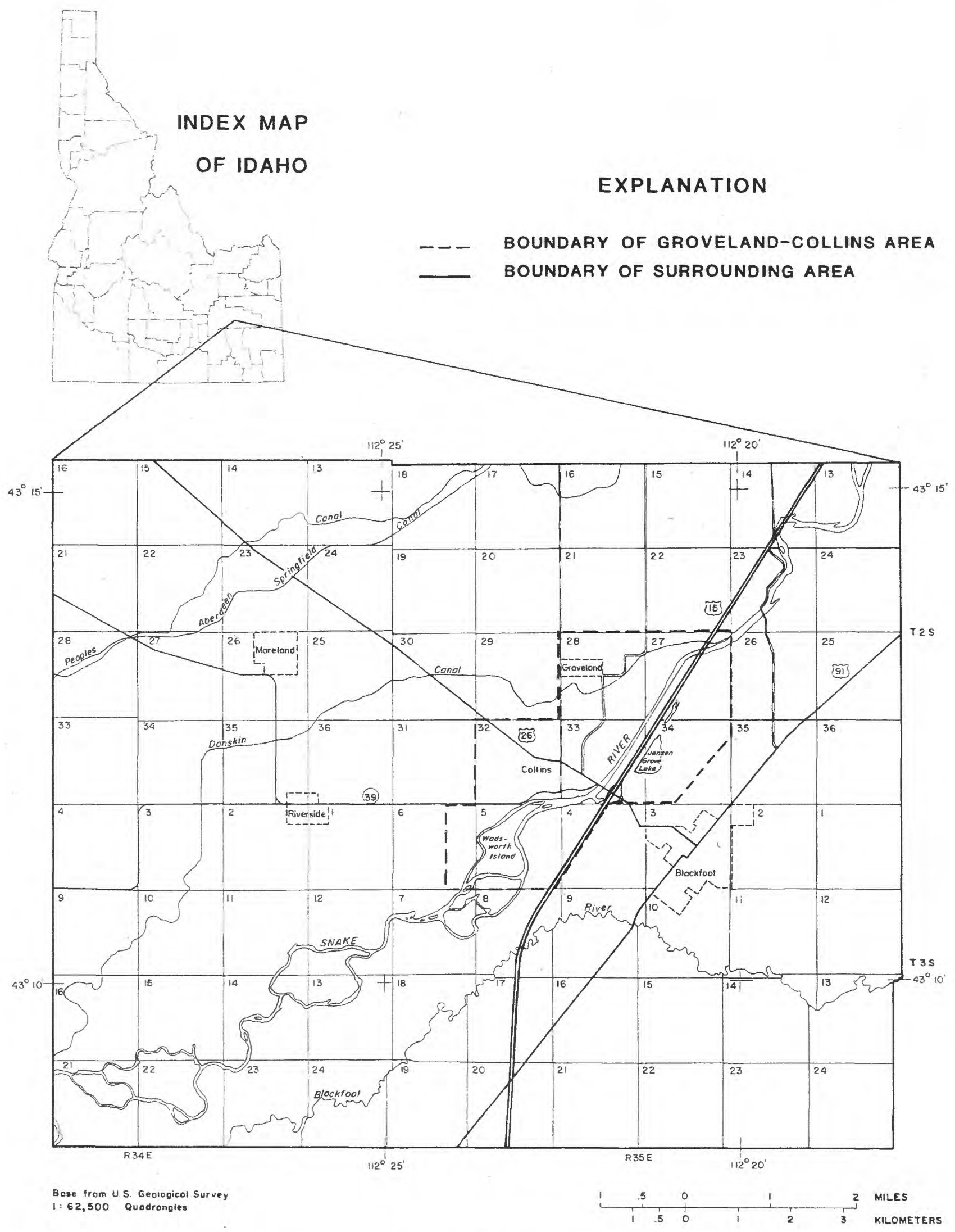

Figure 2.--Location of Groveland-Collins and surrounding areas. 
Selected well-construction, geologic, and water-level data for 163 wells in the study area (fig. 3) are shown in table 1. Wells are listed sequentially by township, range, and section location. Identification of well locations is simplified by use of assigned well numbers.

Well-construction, geologic, and well-use data generally are based on drillers' logs or information obtained from well owners. Water-level data include onsite measurements made by Idaho Department of Water Resources personnel for the period $1978-80$ and by U.S. Geological Survey personnel for the period 1981-85. Selected water-level data from 1978 to 1980 originally were published by Bingham County (1981).

\section{Ground-Water Quality Data, Pre-1984}

Selected pre-1984 water-quality data for 51 wells (fig. 4 ) are listed in table 2. Wells are listed sequentially by township, range, and section location. Identification of well locations is simplified by use of assigned well numbers.

Most pre-1984 ground-water samples were collected by personnel from the Idaho Department of Health and Welfare, Southeastern District Health Department (Blackfoot) or Division of Environment (Pocatello), and were analyzed at the Idaho Department of Health and Welfare Bureau of Laboratories (Boise). Bacterial analyses were provided by the Idaho Department of Health and Welfare Branch Laboratory (Pocatello). Ground-water samples collected by Bingham County personnel were analyzed at various Idaho Department of Health and Welfare laboratories. An asterisk (*) after the sampling date in table 2 denotes an Idaho Department of Health and Welfare laboratory analysis made before the laboratory certification program was instituted (1976-77). U.S. Geological Survey samples were analyzed at the U.S. Geological Survey Central Laboratory (Denver). Unless noted otherwise, reported values are dissolved constituents and laboratory analyses, not onsite analyses.

Additional bacterial analyses for selected water samples are available in a report by Forsgren-Perkins Engineering (1982) but are not included in table 2 . 


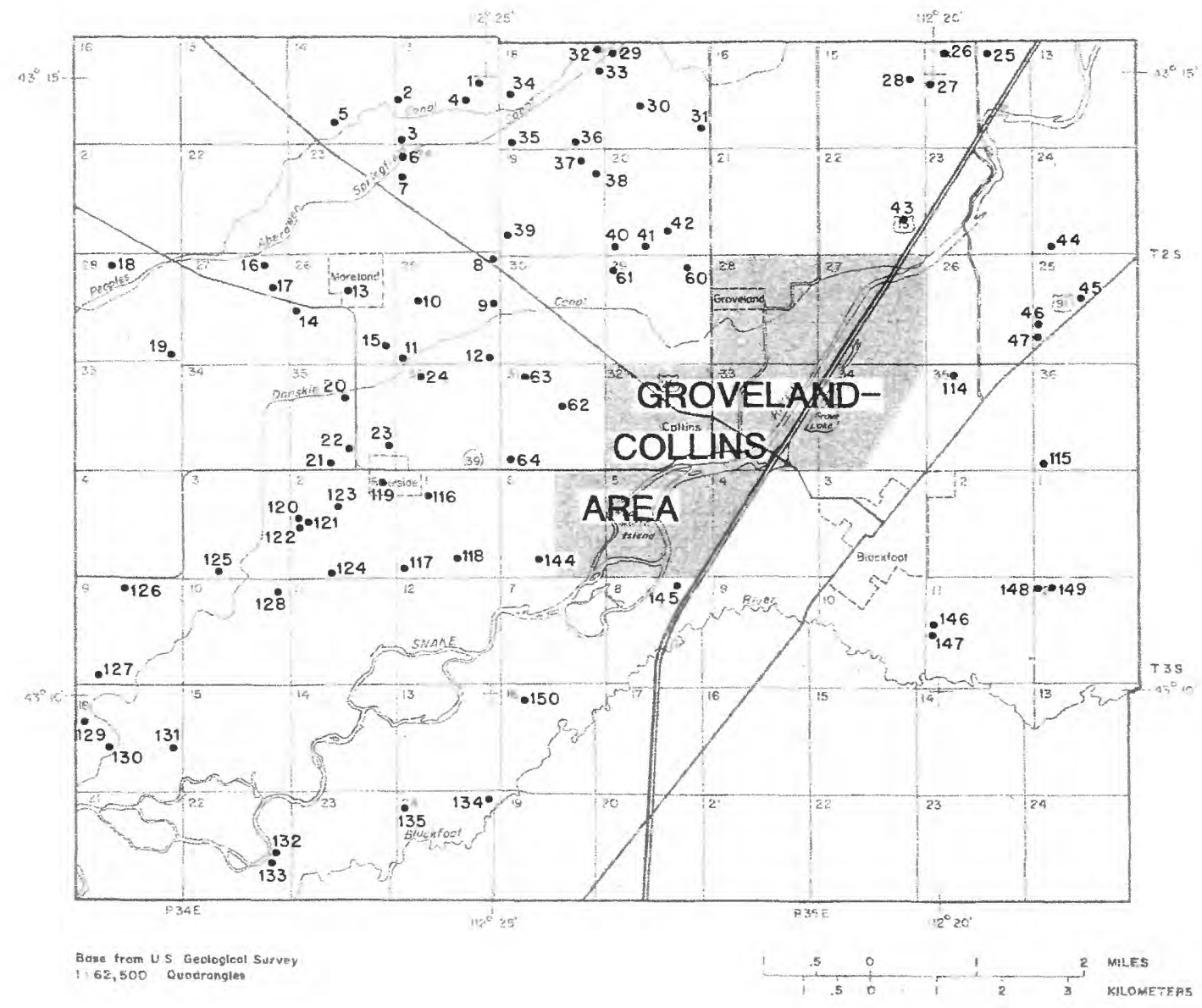

DETAIL OF GROVELANDCOLLINS AREA

(shaded area above)
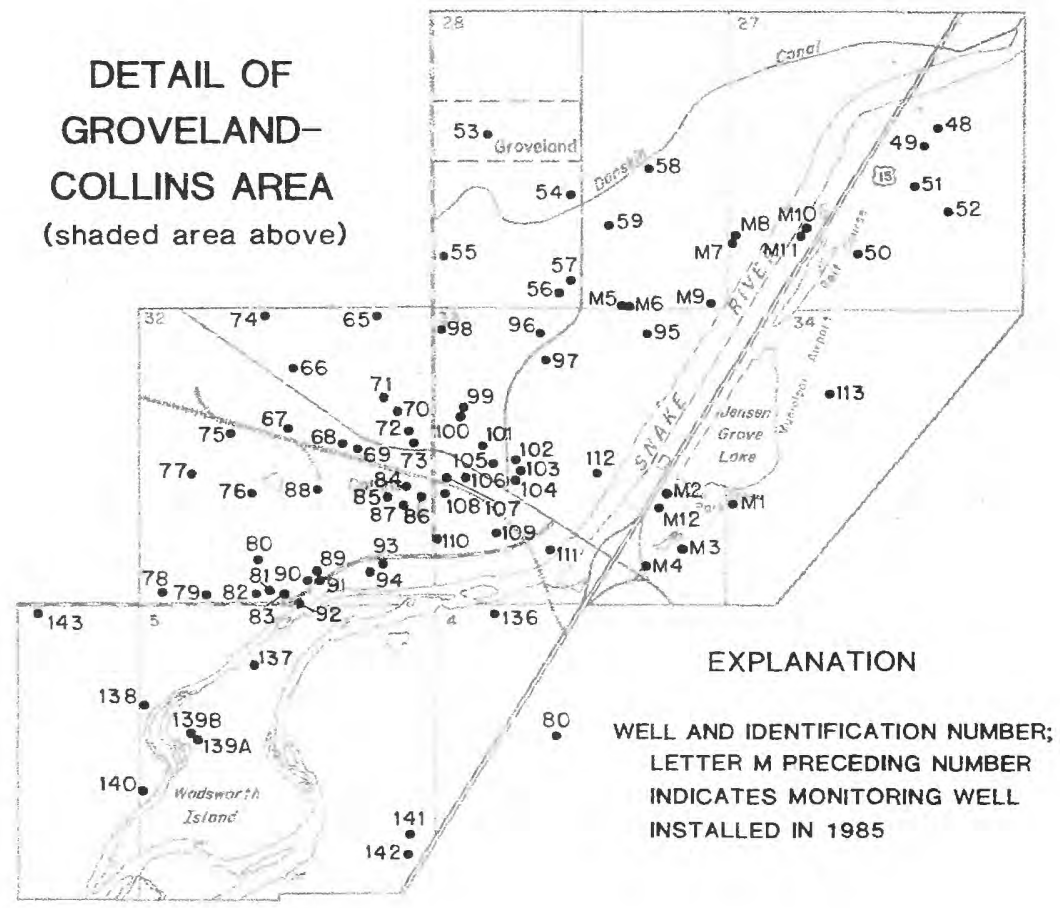

Figure 3.--Locations of wells selected for use during 1984-85 study. 
During 1984 and 1985, water-quality samples were collected from 54 wells (fig. 4) by U.S. Geological Survey (Boise) personnel. Onsite and laboratory analyses of these samples are shown in table 3 . Wells are listed sequentially by township, range, and section location. Identification of well locations is simplified by use of assigned well numbers.

Because certain water-quality characteristics may change between time of sample collection and laboratory analysis, onsite determinations of the following characteristics were made: water temperature, $\mathrm{pH}$, specific conductance, bicarbonate and carbonate concentrations (endpoint titration method), and dissolved-oxygen concentration (membrane electrode, onsite calibration method).

Methods used for collection and preservation of samples and onsite water-quality determinations are described in reports by U.S. Geological Survey (1977), Feltz and others (1983), and Feltz and Anthony (1984). Onsite equipment included Sybron/Barnstead ${ }^{1}$ conductivity bridge, Sargentwelch $\mathrm{pH}$ meter with Sensorex sealed $\mathrm{pH}$ probe, Yellow Springs Instrument oxygen meter and probe, Millipore 0.45-micrometer average pore-diameter cellulose nitrate membrane filter, and Flotronics 0.45-micrometer silver filter. Laboratory analyses were completed by the U.S. Geological Survey Central Laboratory (Denver). Data collected onsite are noted by an asterisk in table 3 column headings; calculated constituents are defined in table 3 headnotes.

\footnotetext{
${ }^{1}$ Use of brand and trade names in this report is for identification purposes only and does not constitute endorsement by the U.S. Geological Survey.
} 

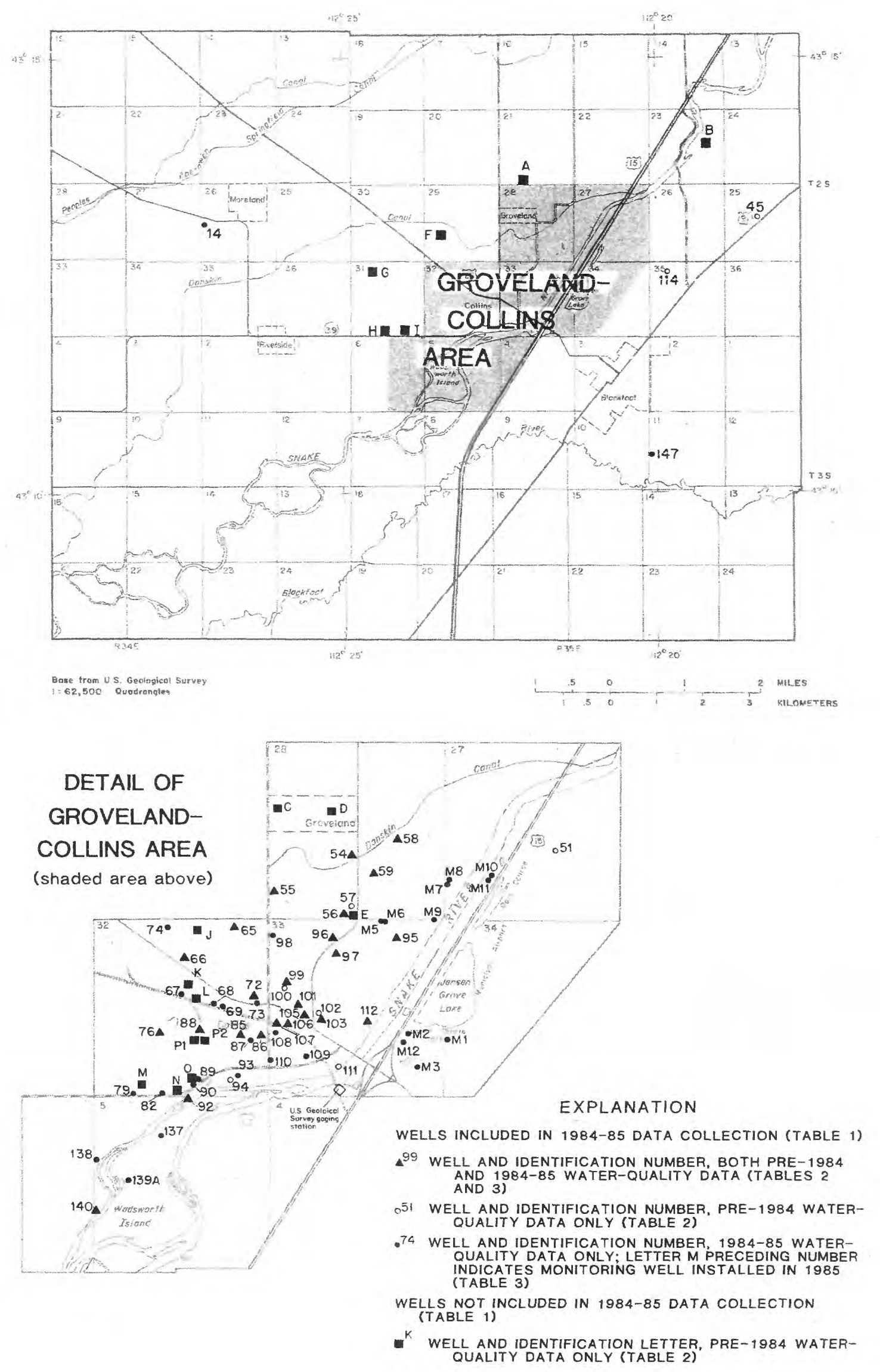

Figure 4.--Locations of wells with selected pre-1984 and 1984-85 water-quality data. 
Bingham County, contractor, 1981, Bingham County groundwater project: Blackfoot, Idaho, U.S. Environmental Protection Agency Grant, Water Quality Program, $414 \mathrm{p}$.

Feltz, H. R., and Anthony, E. R., eds., 1984, 1985 Water quality laboratory services catalog: U.S. Geological Survey Open-File Report 84-171.

Feltz, H. R., Anthony, E. R., and Sadler, P., eds., 1983, 1984 Water quality laboratory services catalog: U.S. Geological Survey Open-File Report 83-758.

Forsgren-Perkins Engineering, 1982, Bingham County westside study for the communities of Collins, Groveland, Moreland, and Riverside: Rexburg, Idaho, a portion of U.S. Environmental Protection Agency Project no. C-16 0371-01, 120 p., 6 apps.

Price, W. E., and Baker, C. H., 1974, Catalogue of aquifer names and geologic codes used by the water Resources Division: U.S. Geological Survey, $306 \mathrm{p}$.

U.S. Geological Survey, 1977, National handbook of recommended methods for water data acquisition: Reston, Va., Office of Water Data Coordination, Chap. 1-12.

1983, WATSTORE user's guide: Reston, Va., v. 3 (Water-quality File), Chap. VI-A, 26 p. 
Table 1.--Well and water-level data

Headnotes

MAJOR AQUIFER:

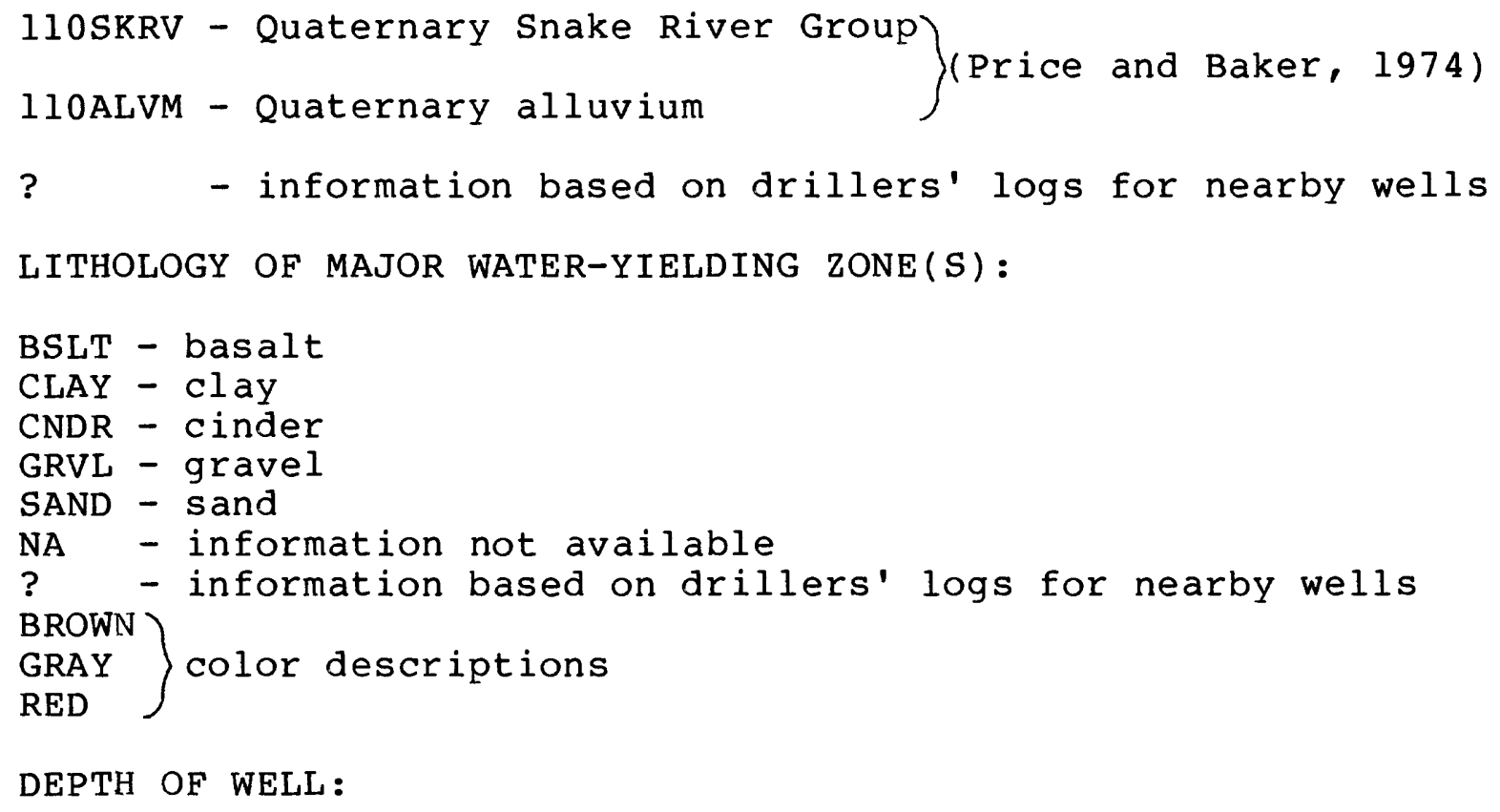


ALTITUDE OF LAND SURFACE: Based on land level survey or interpolated from topographic maps

DATE WATER LEVEL MEASURED: year-month-day

DEPTH BELOW LAND SURFACE (WATER LEVEL):

no symbol - static (no recent nearby pumping)

$P$ - pumping

$R$ - recently pumped

S - nearby pumping

$T$ - nearby recently pumped

$\mathrm{V}$ - foreign substance, generally oil

ALTITUDE OF TOP OF BASALT:

NA - information not available

? - information based on drillers' logs for nearby wells 


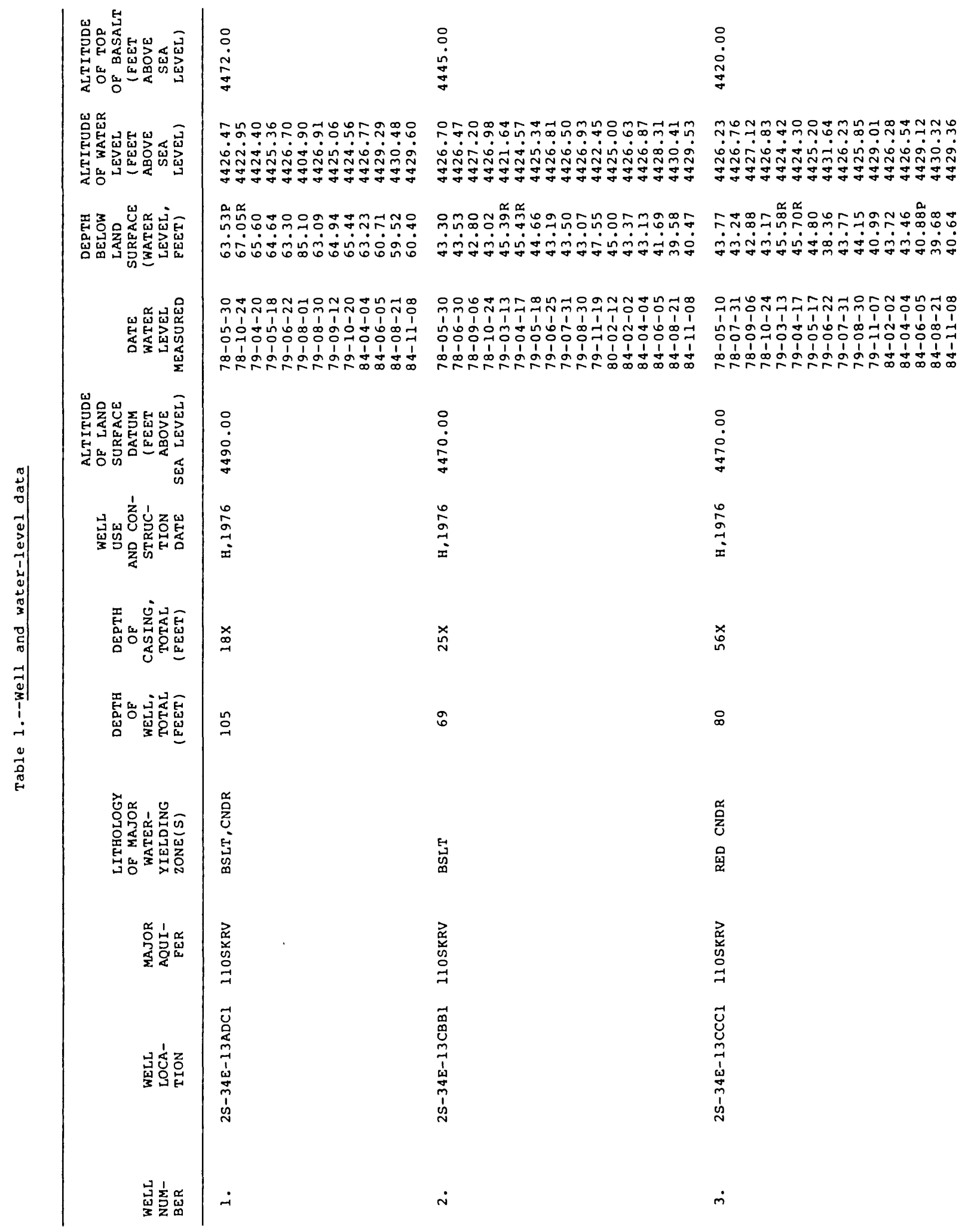




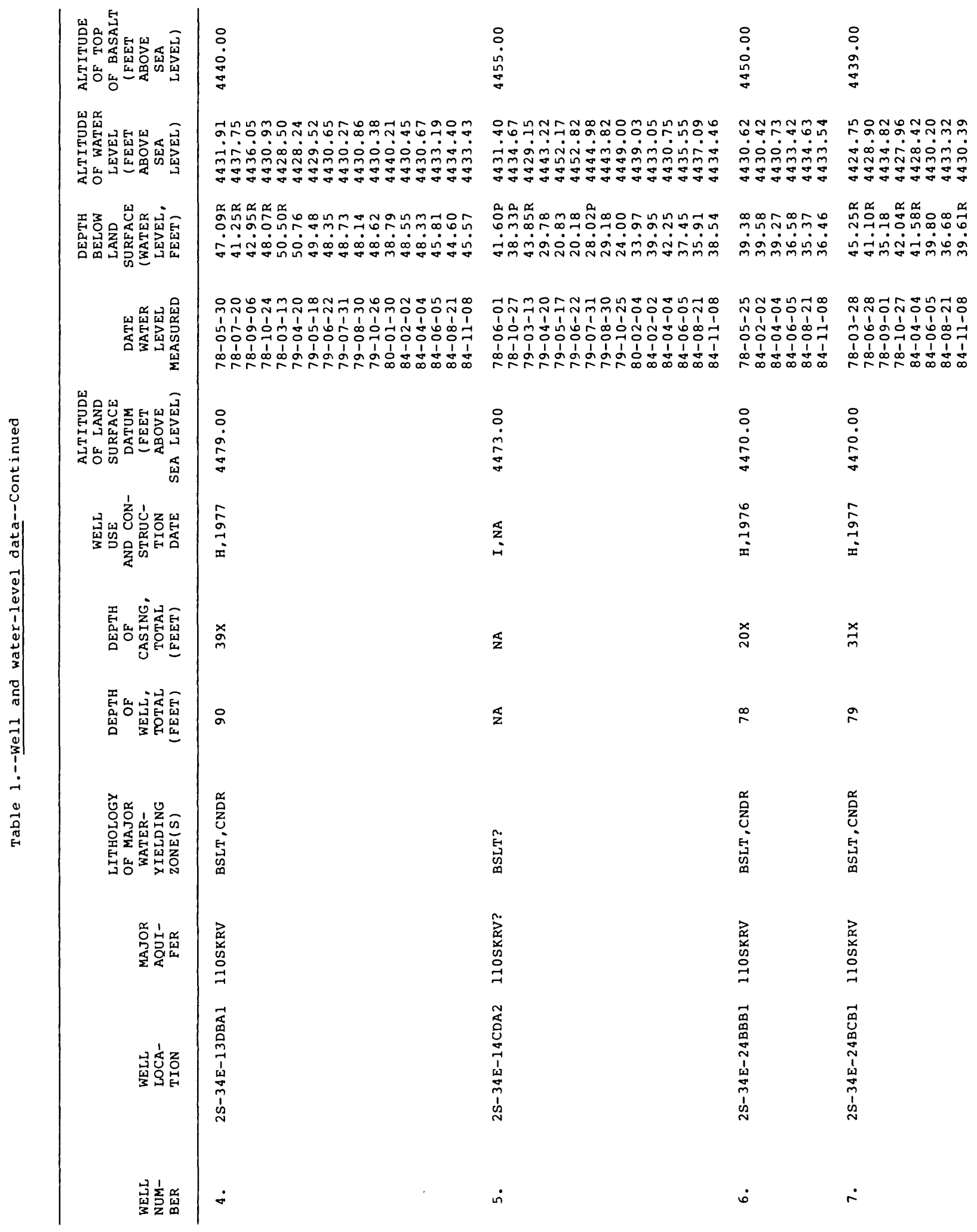




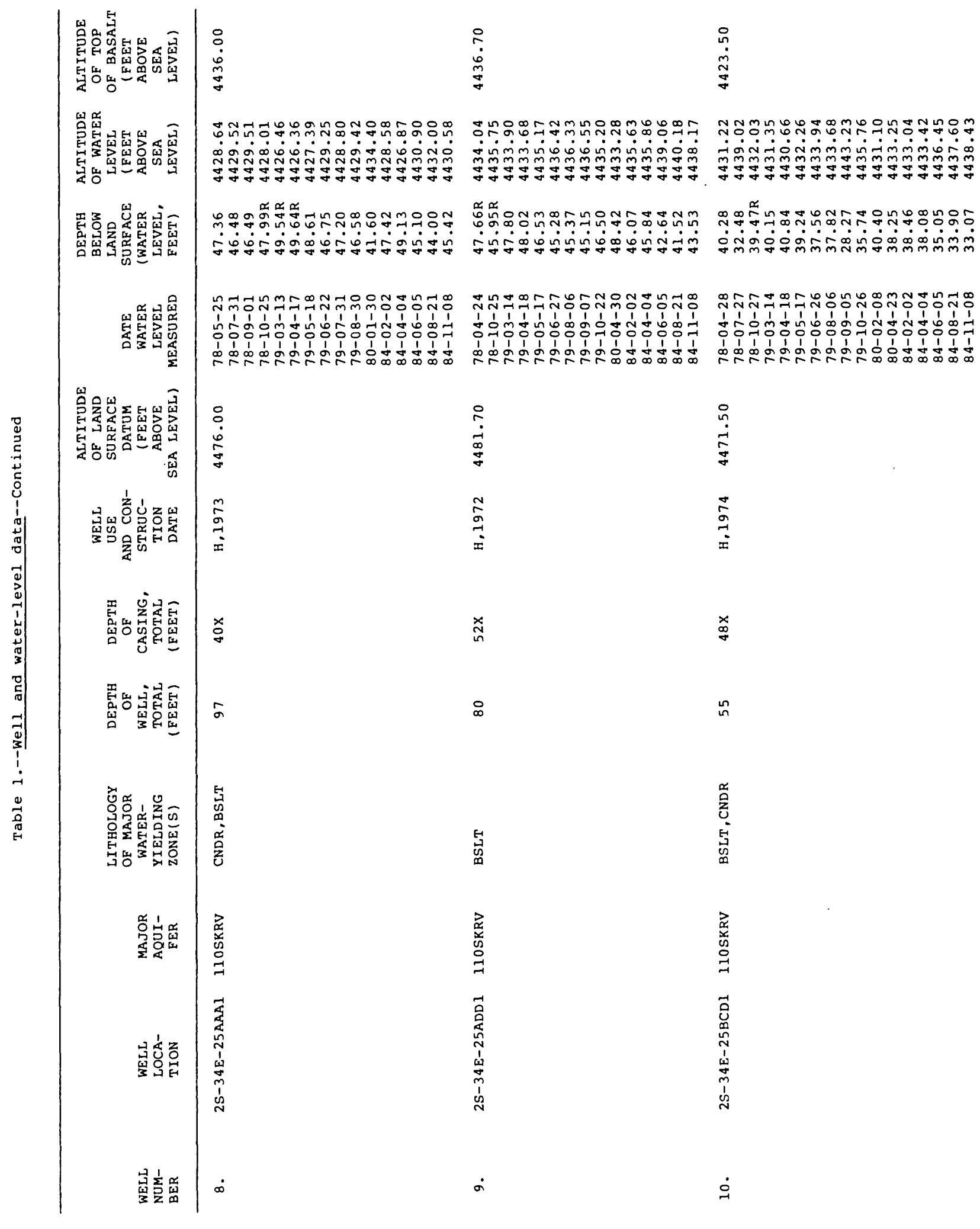




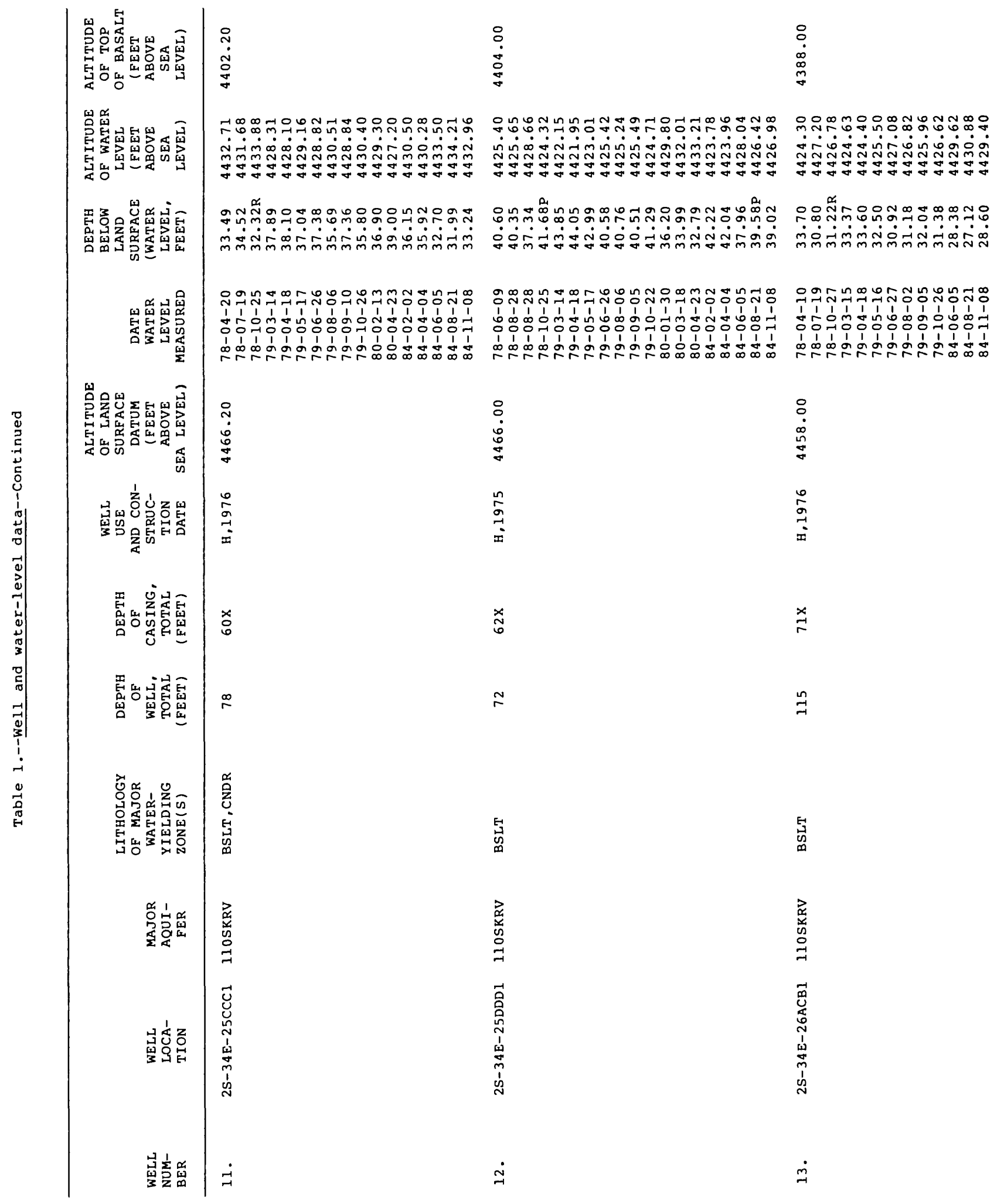




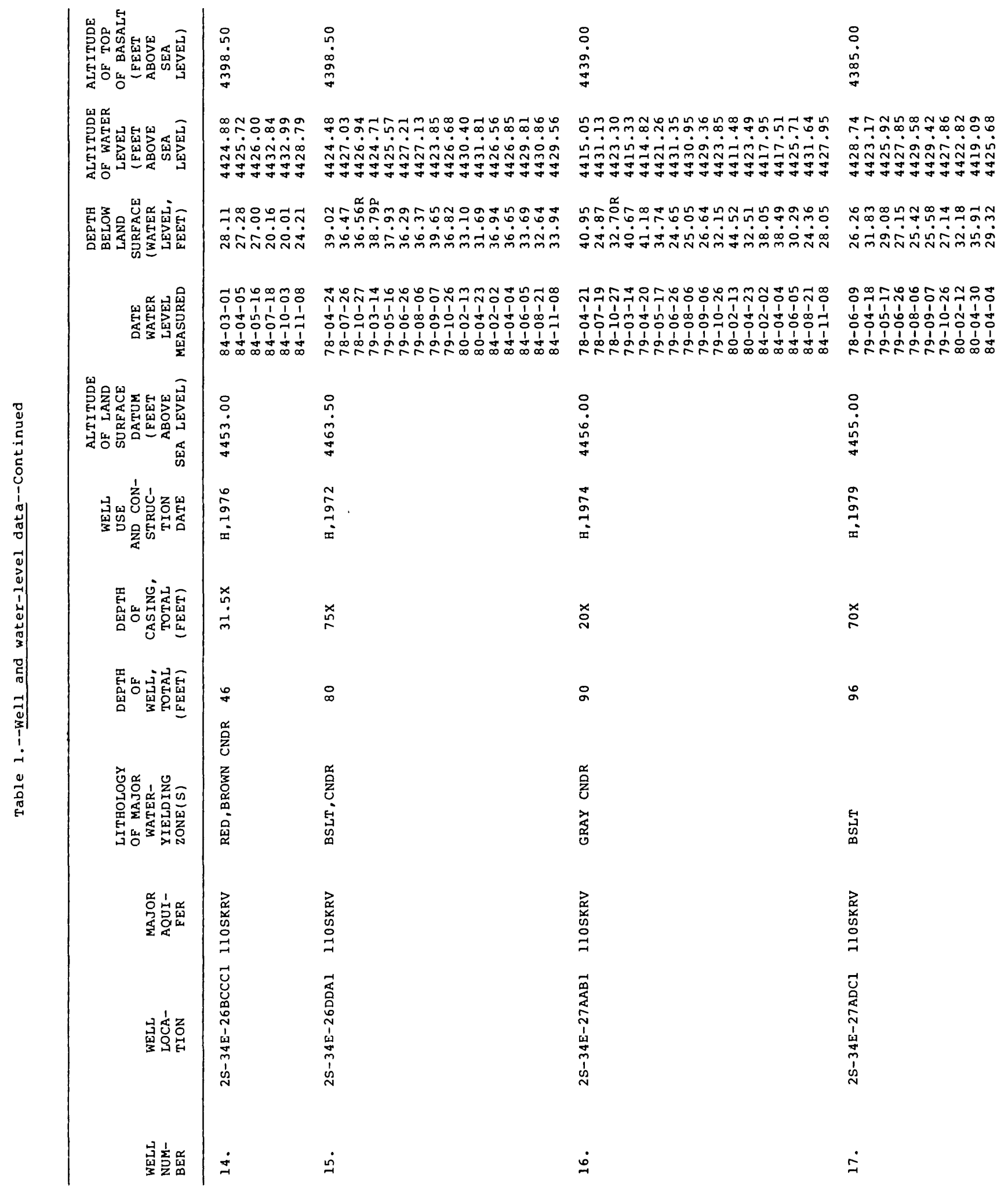




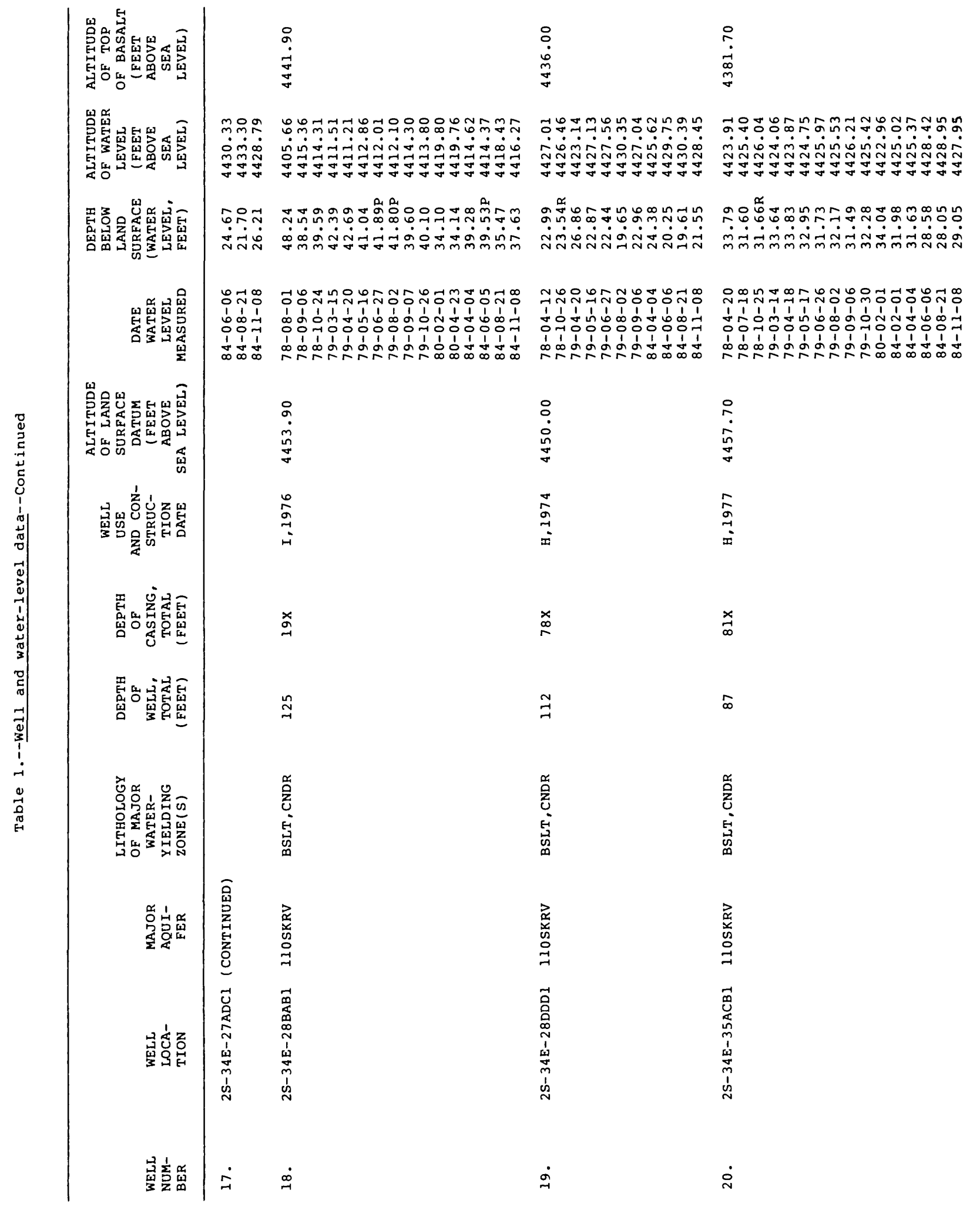




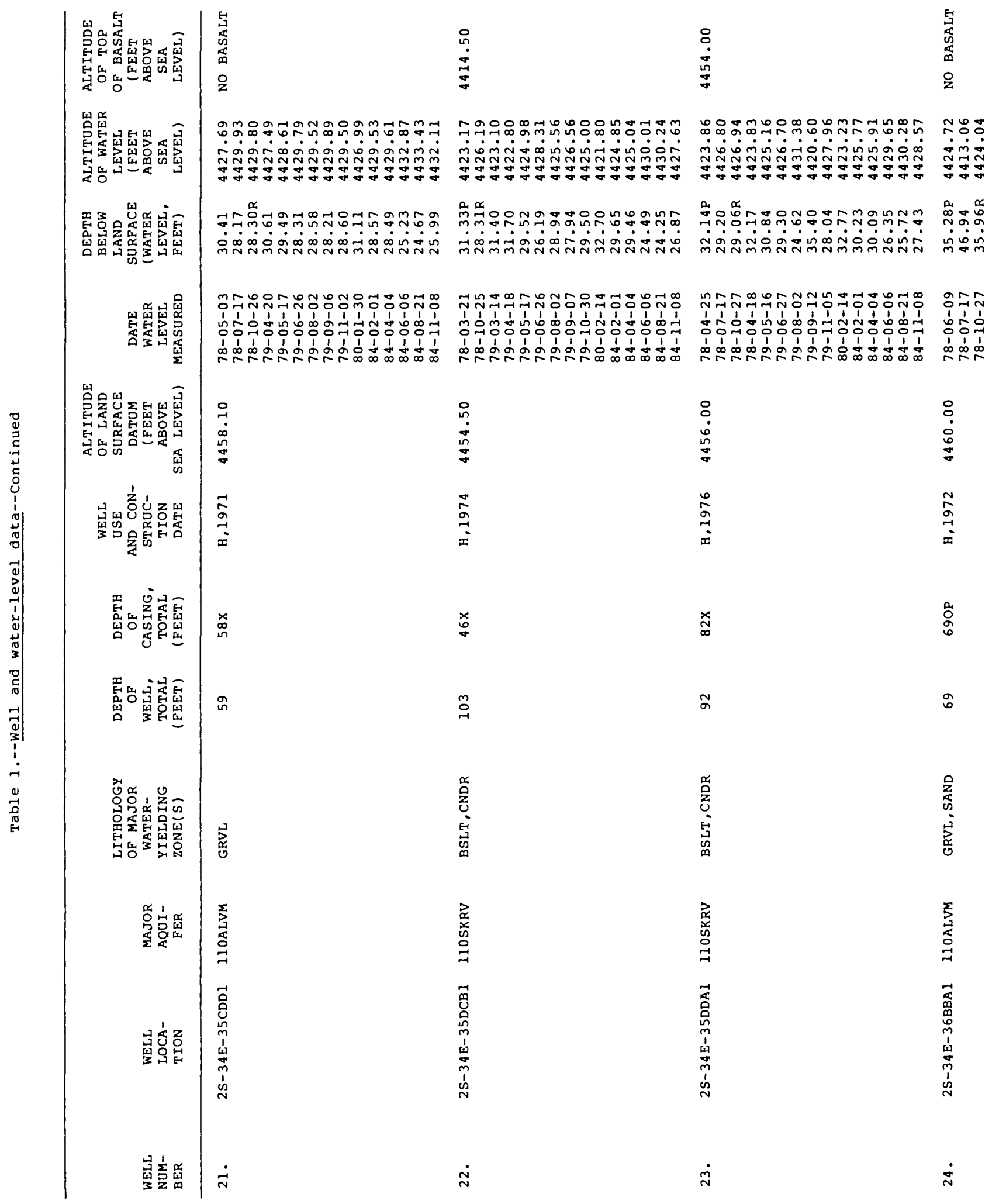



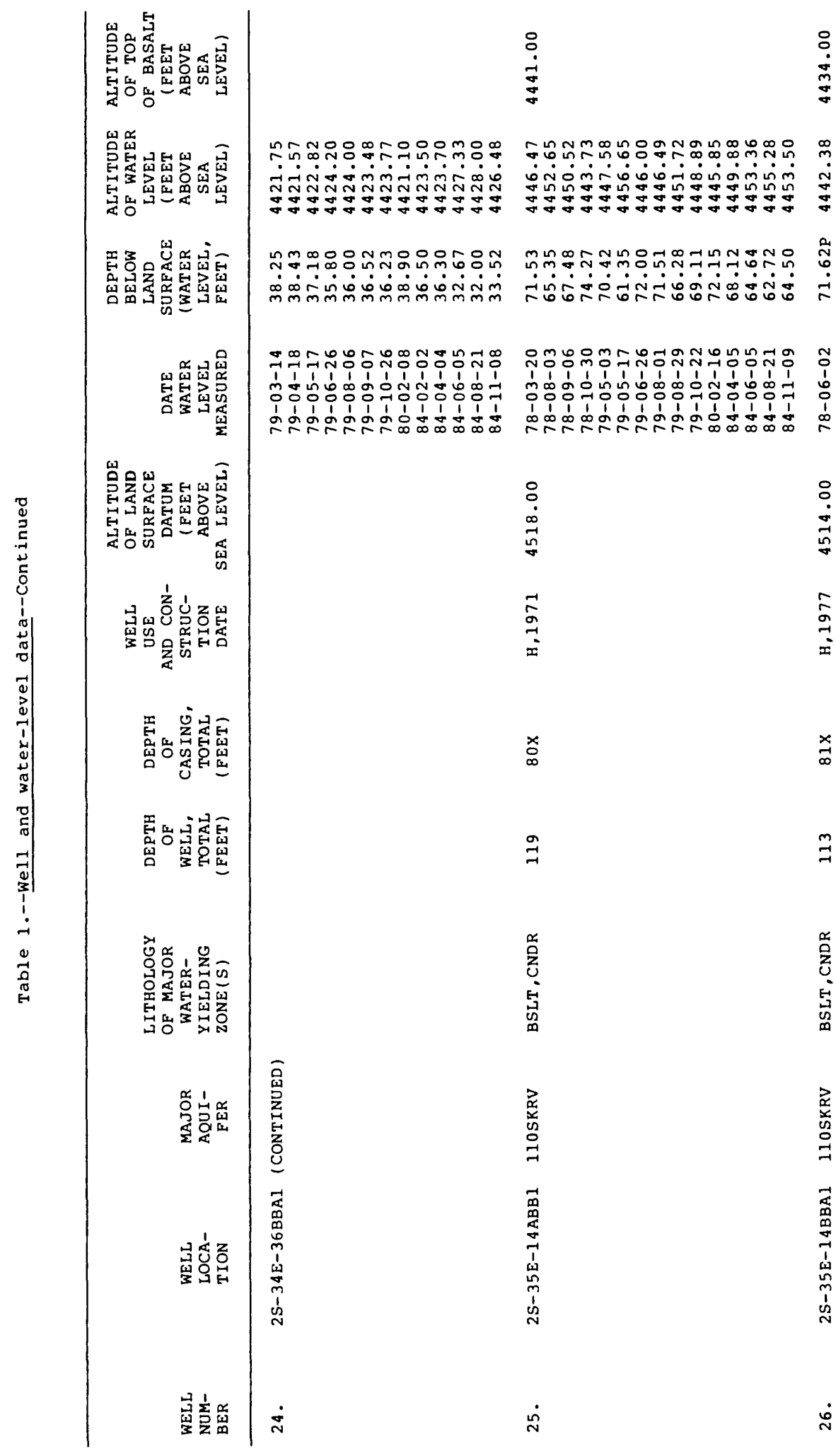

$\stackrel{0}{0}$
$\dot{\alpha}$
$\frac{\sigma}{\sigma}$

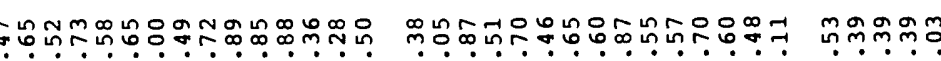

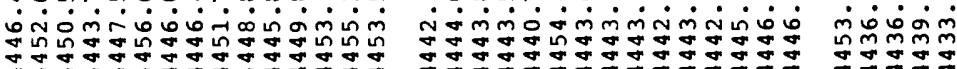
กิ๊ -

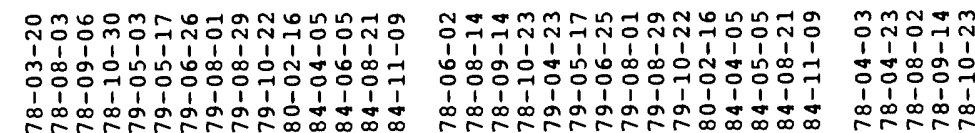

$\circ$
0
$\infty$
01

:

8
$\dot{m}$
$\stackrel{n}{*}$

$\underset{2}{a}$

$\stackrel{2}{a}$

$\underset{n}{N}$

$\underset{\infty}{\infty}$

$\underset{\infty}{\stackrel{x}{1}}$

$\stackrel{\times}{a}$

$\Xi$

$\stackrel{m}{=}$

$\infty$

号

虽

哭

$\overrightarrow{1}$
0
0
0
0
$=$

章

3
0
0
0
0
-1
-1

票

舀

要

$\dot{n}$

$\dot{\sim}$

$\dot{N}$ 


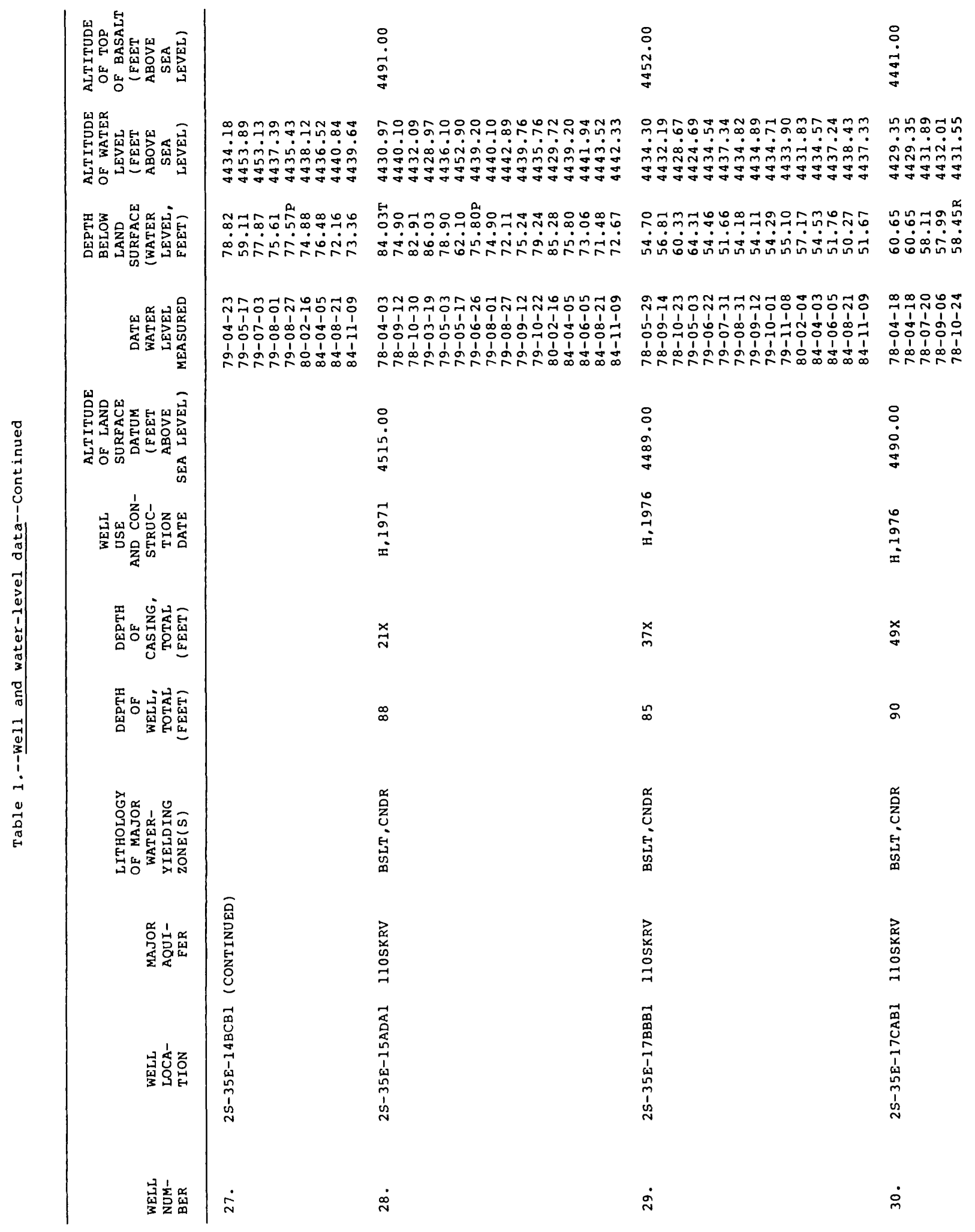




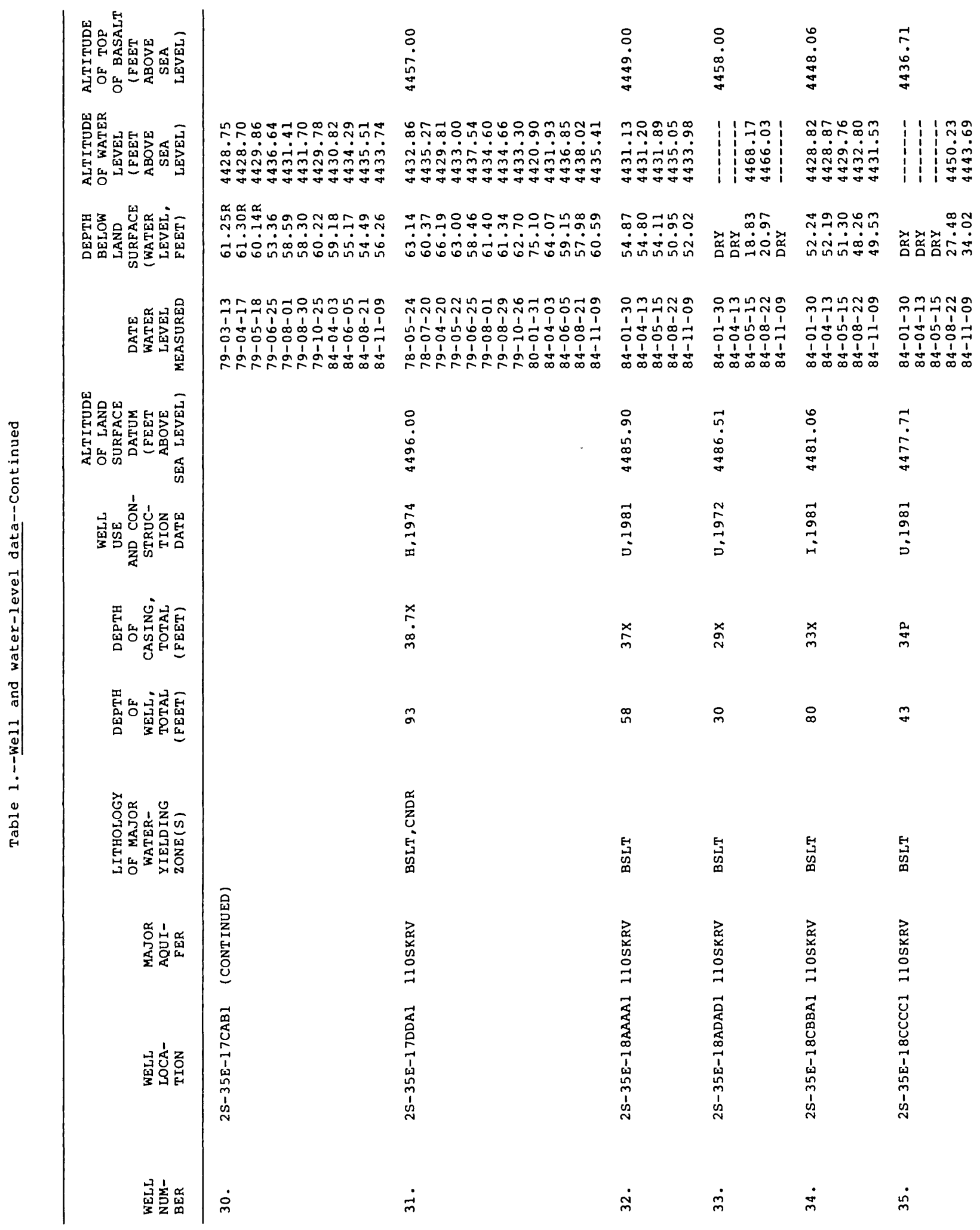




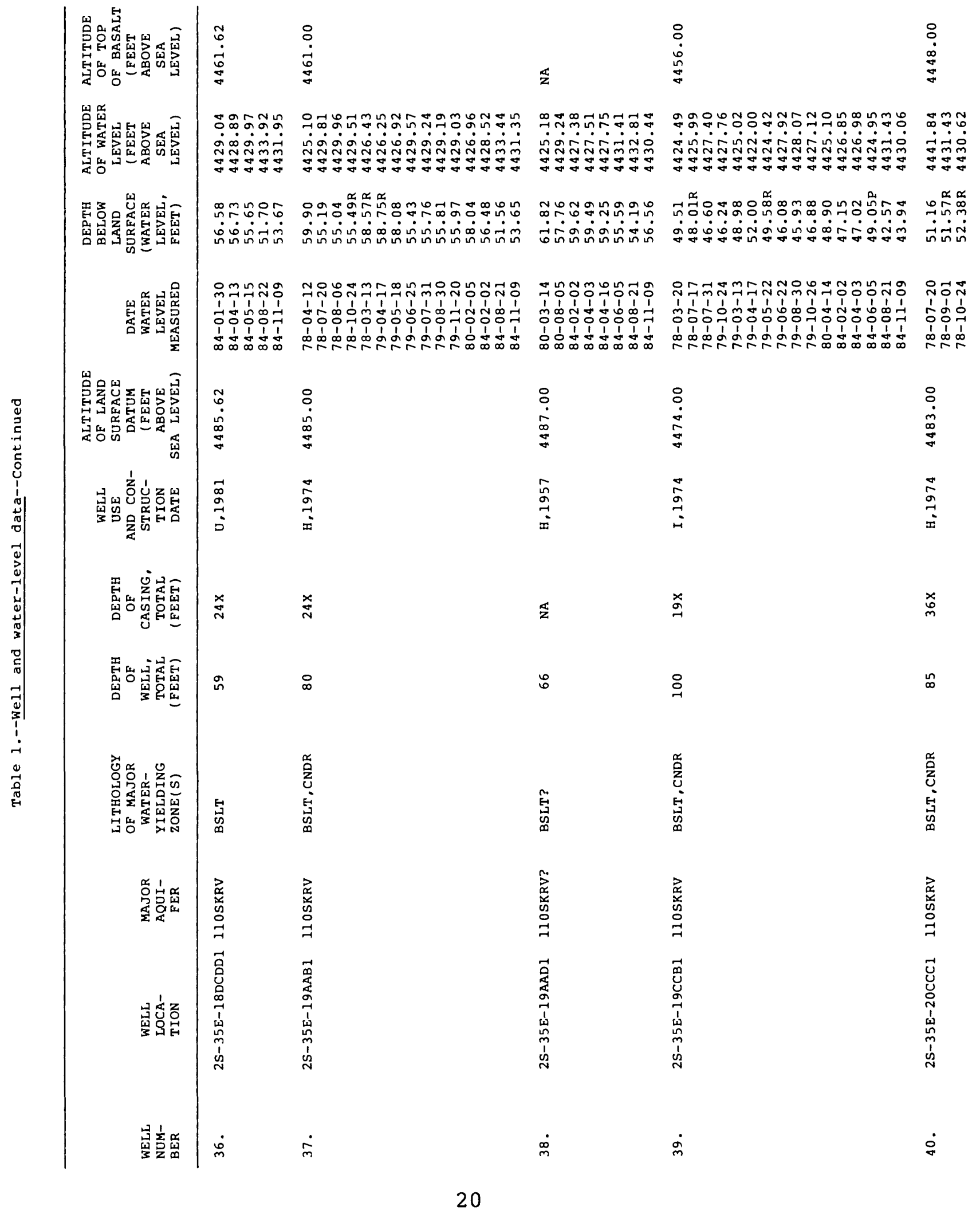




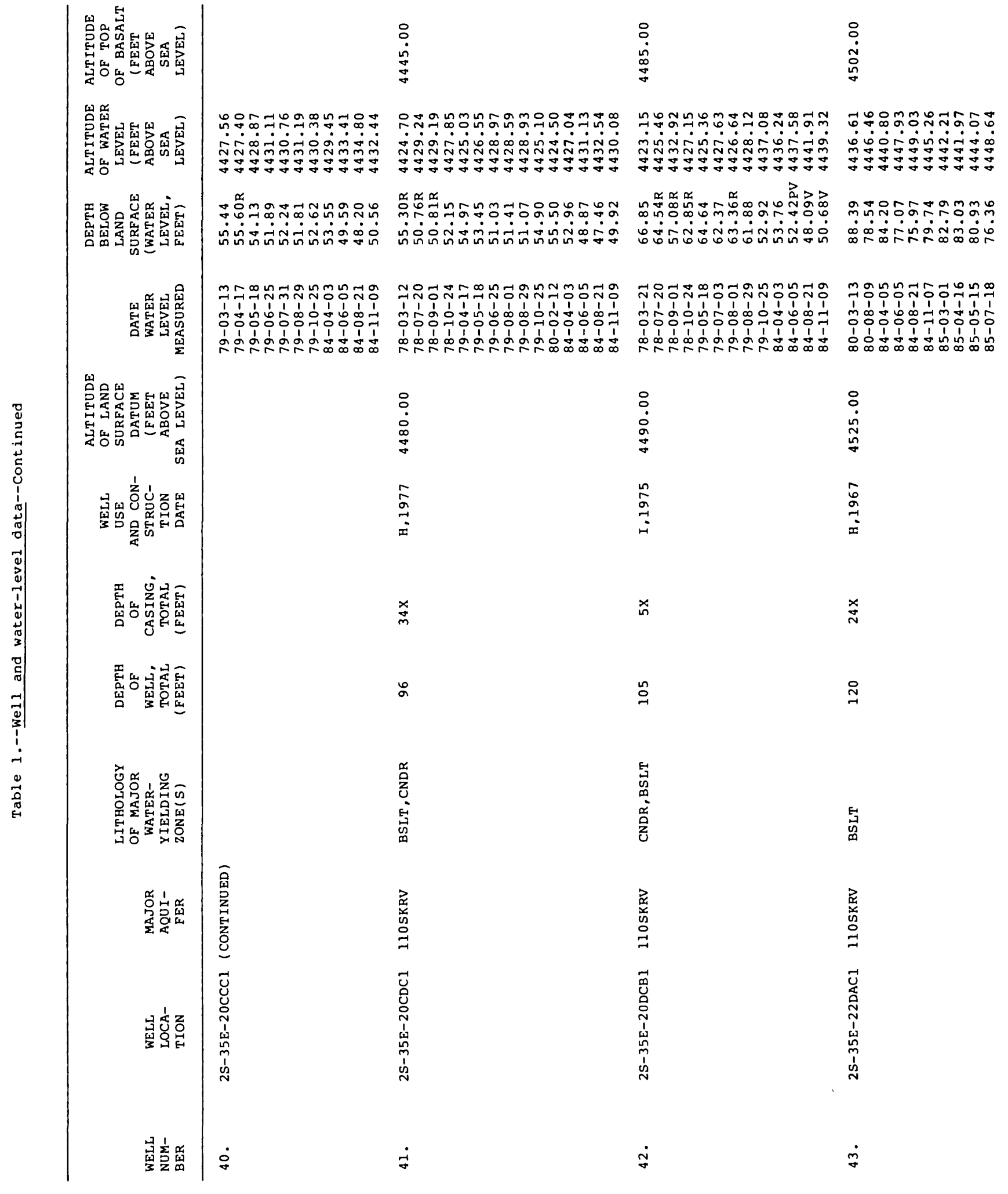




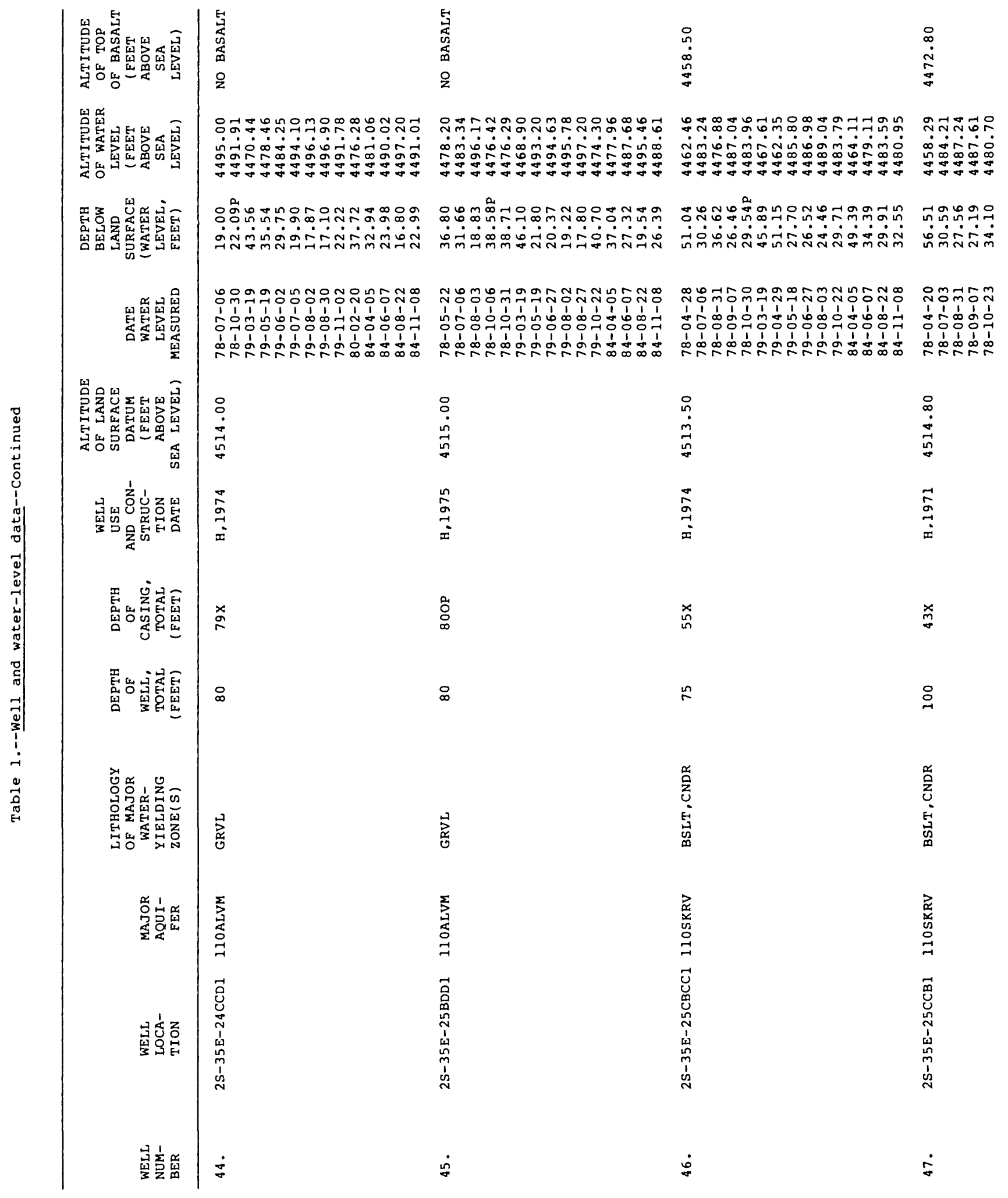




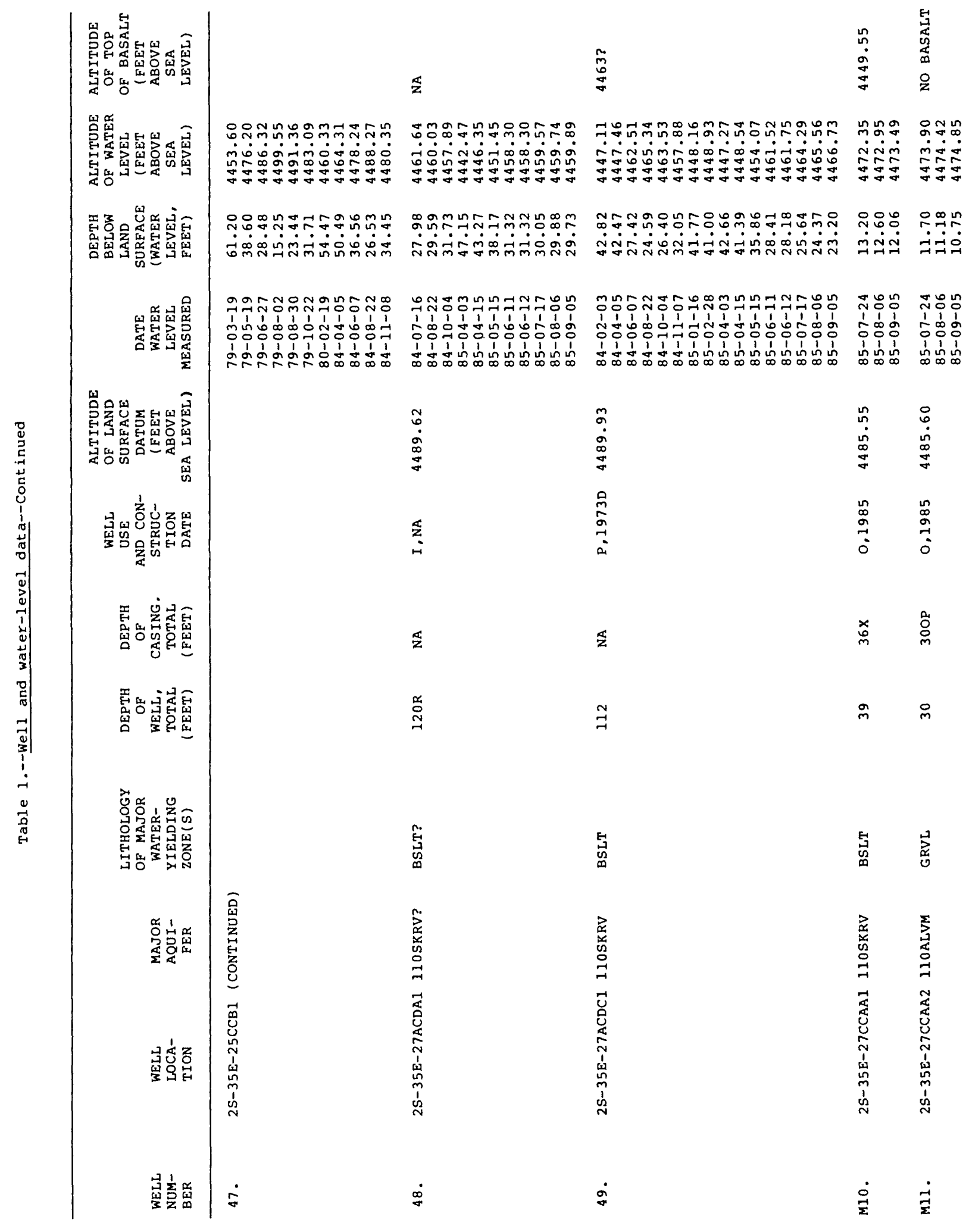




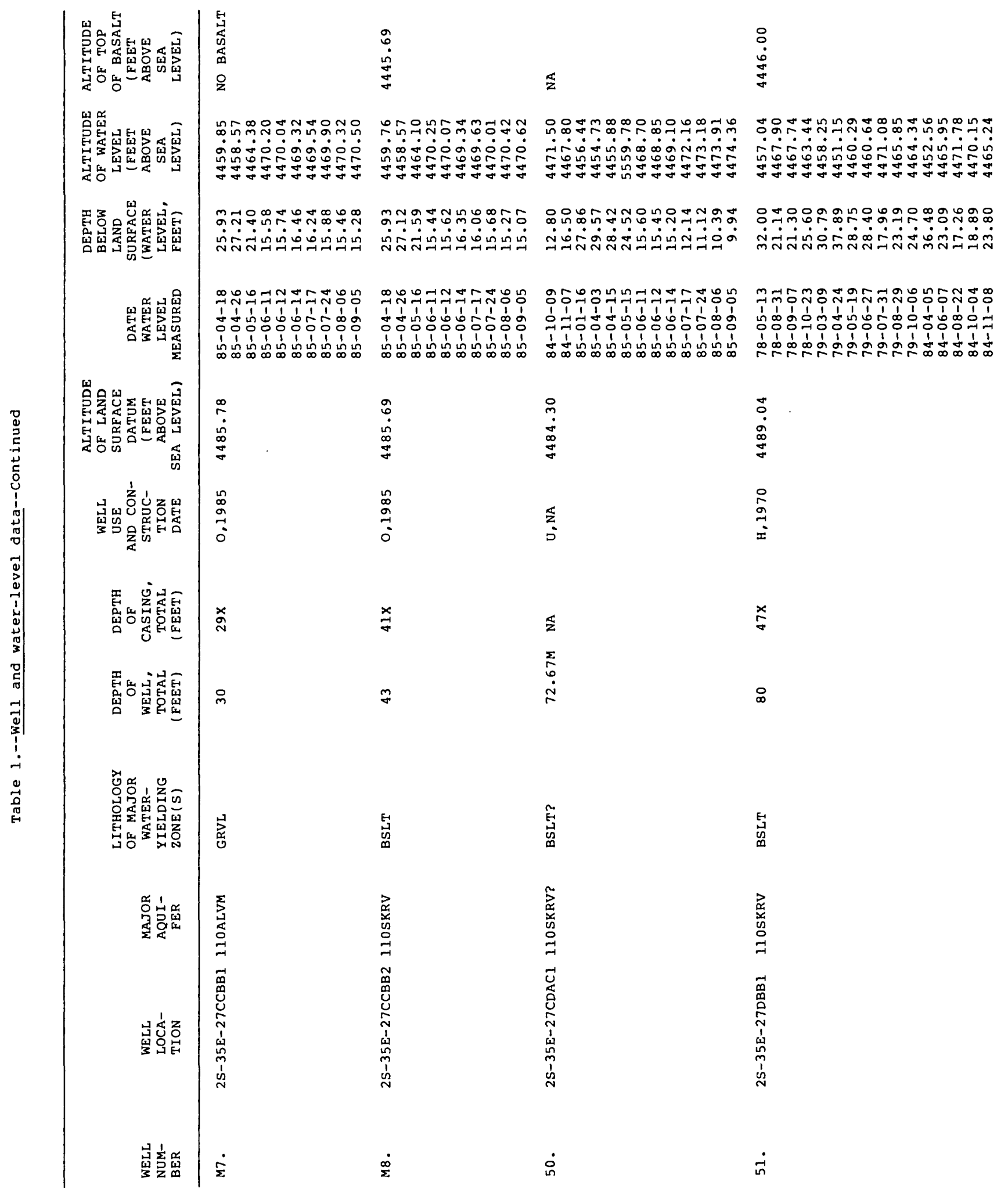




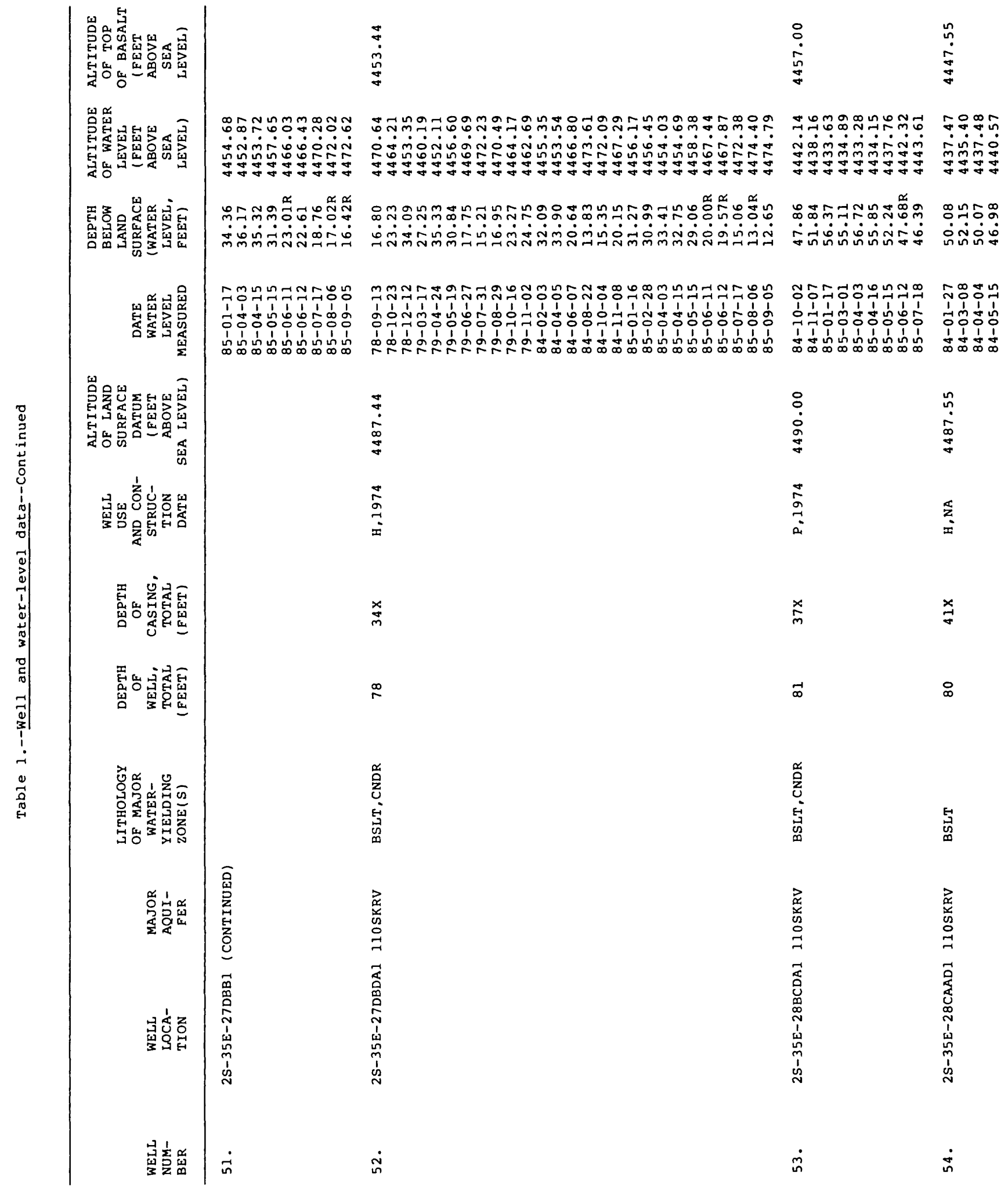




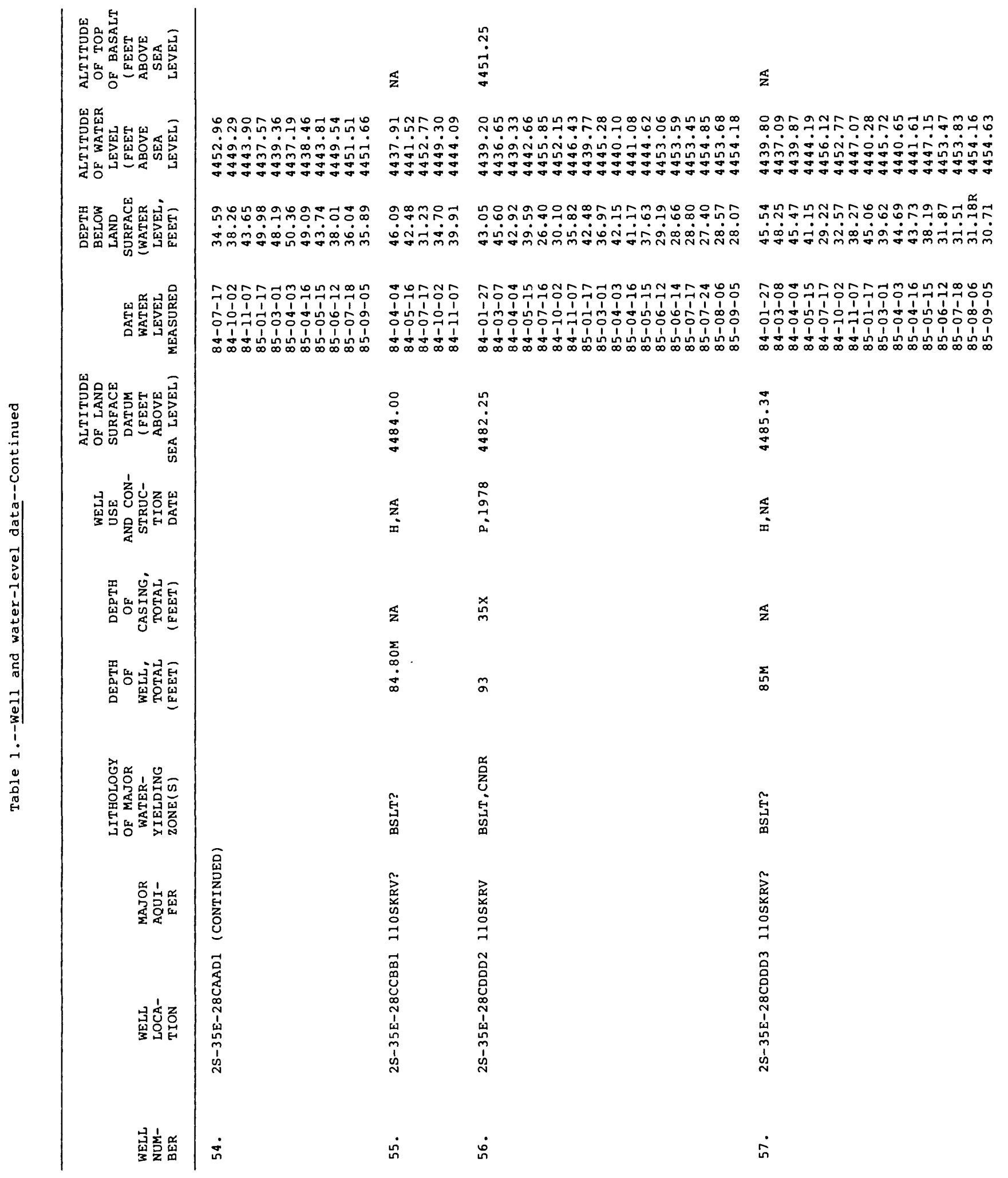




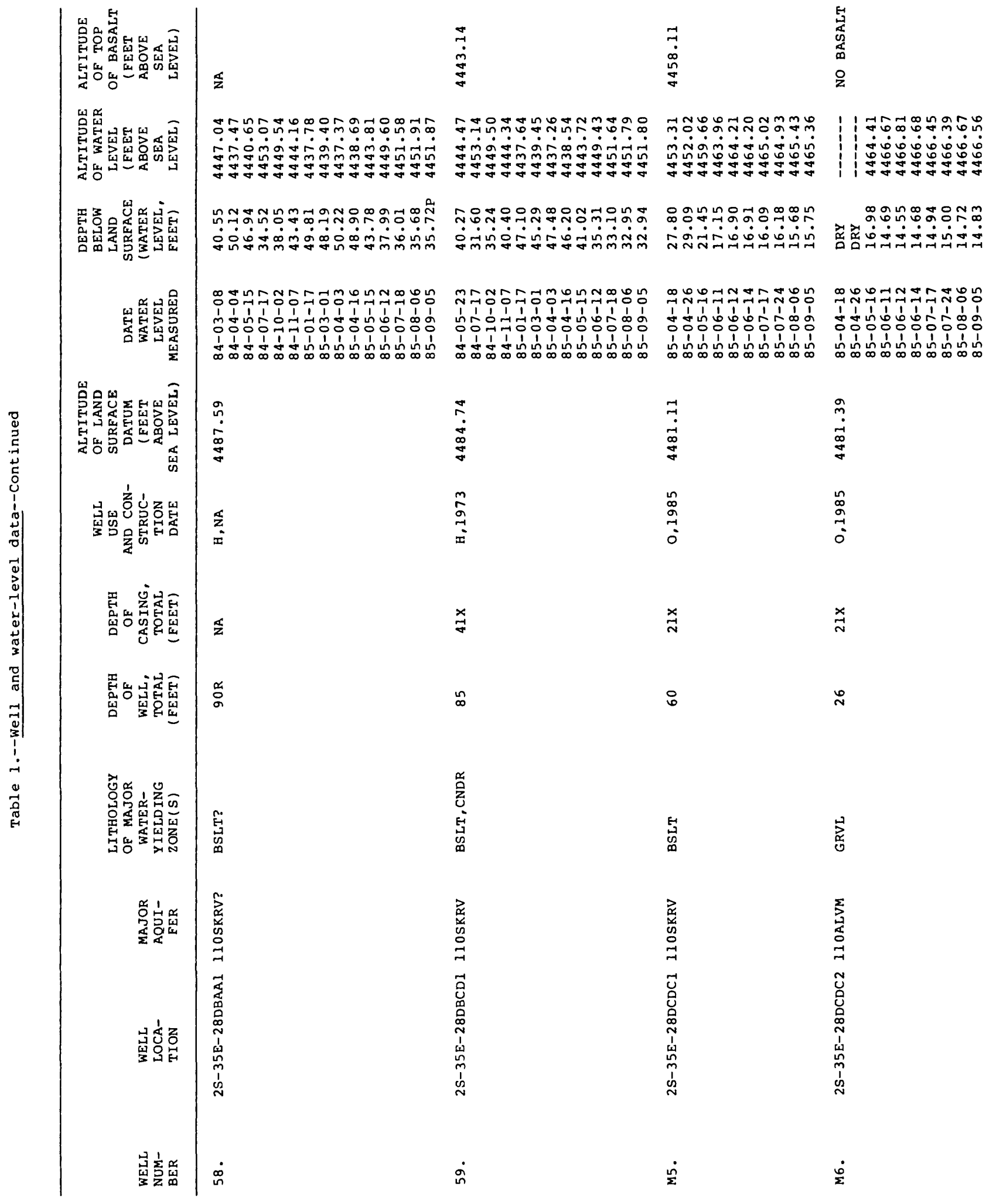




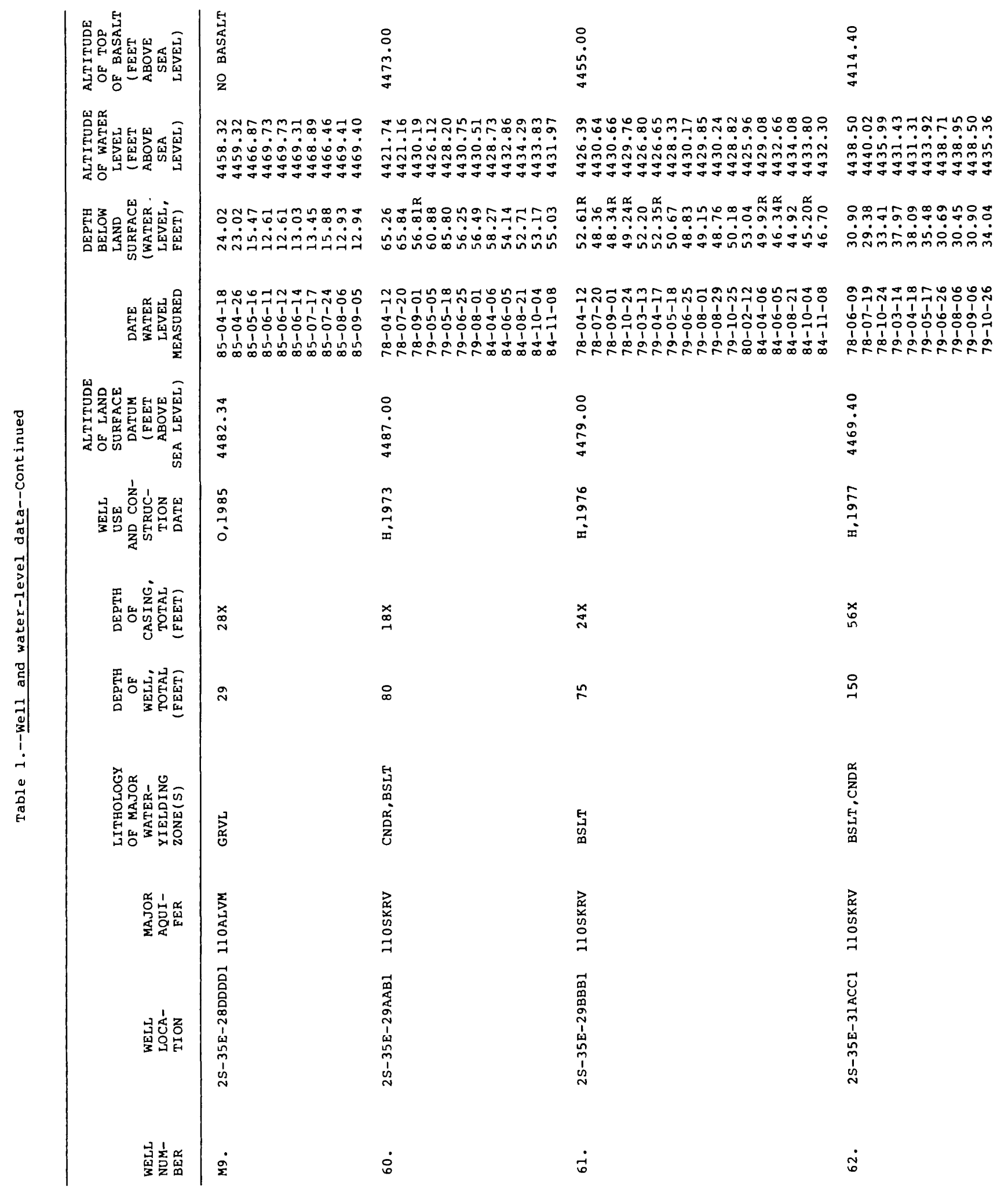




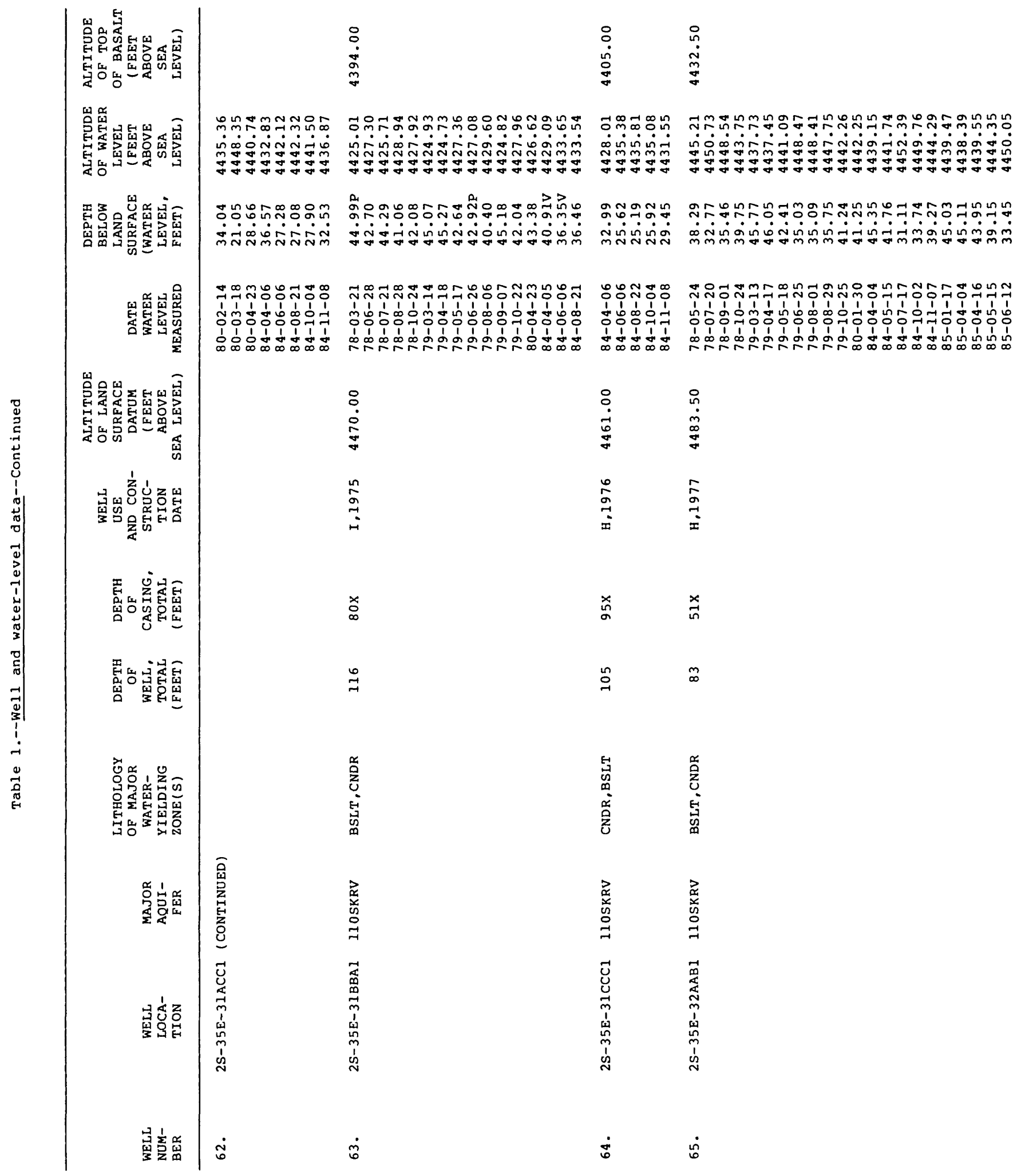




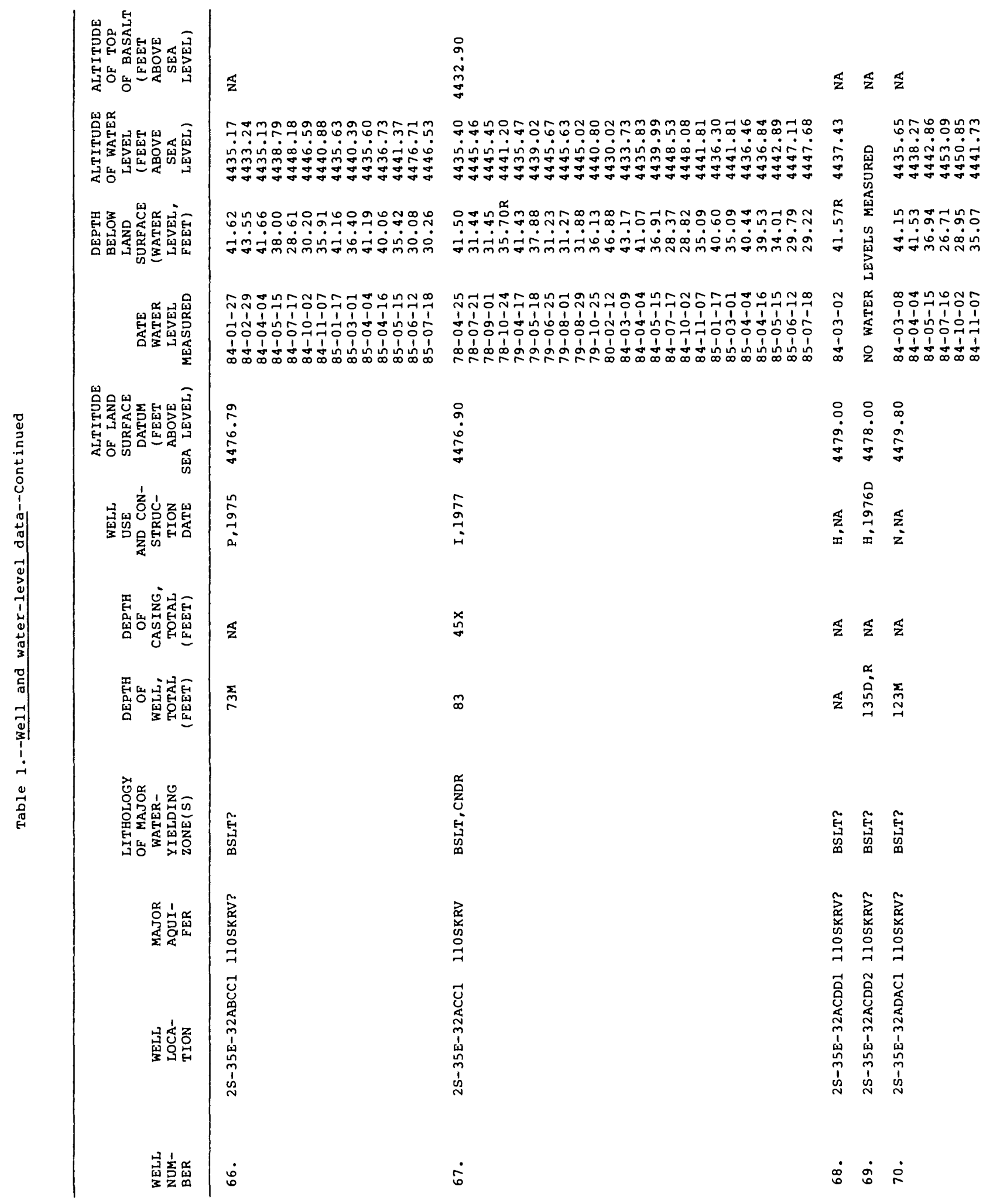




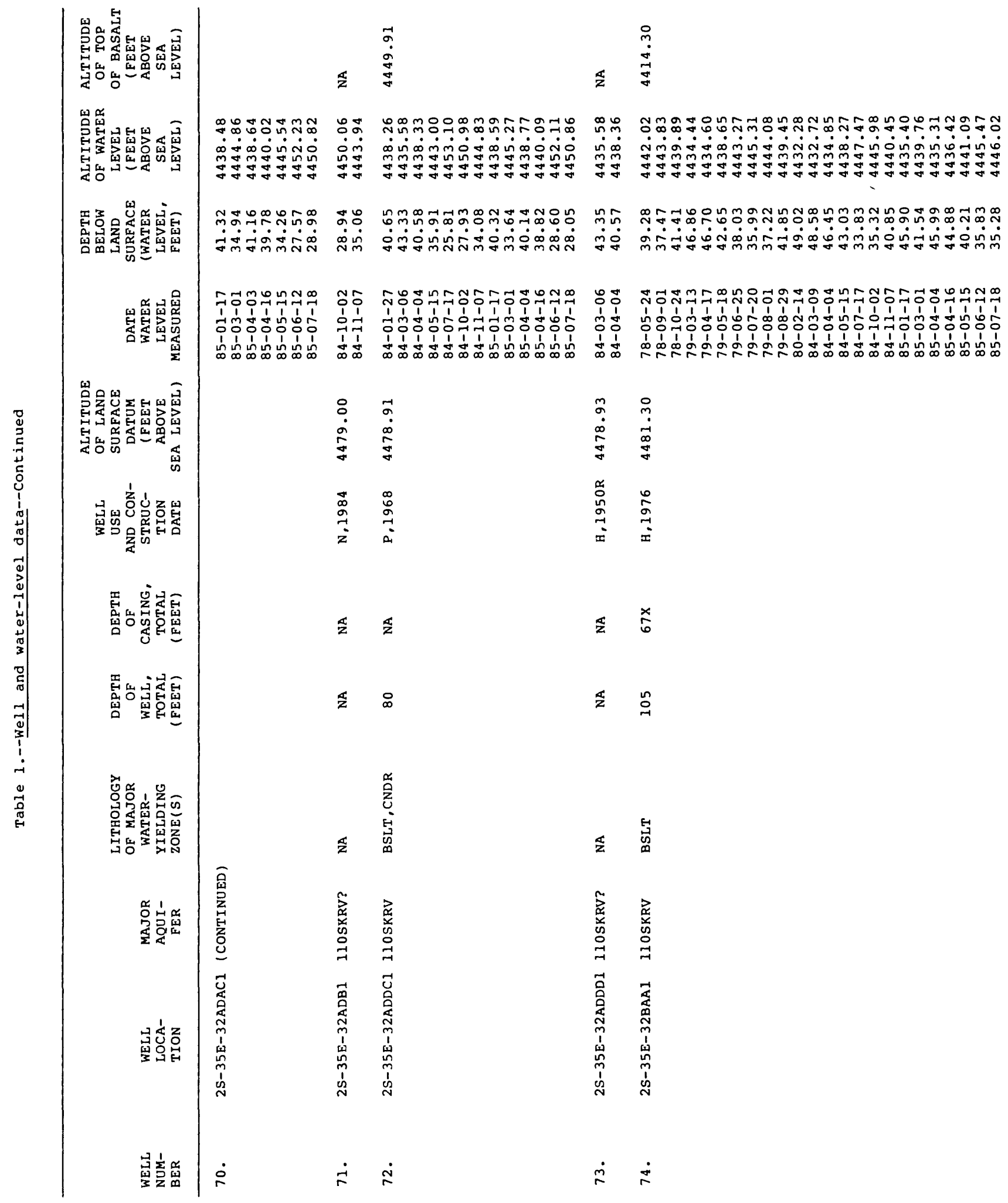




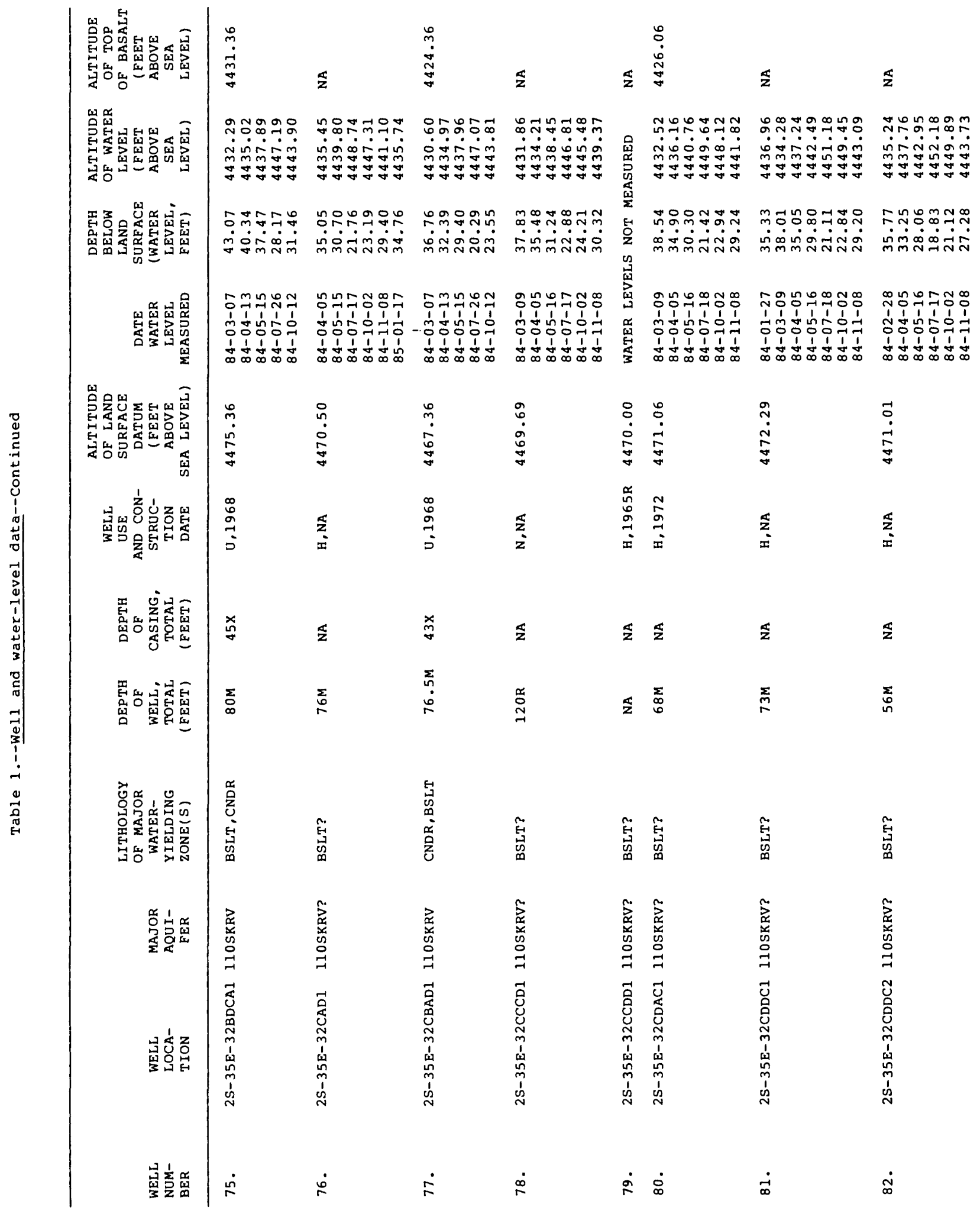




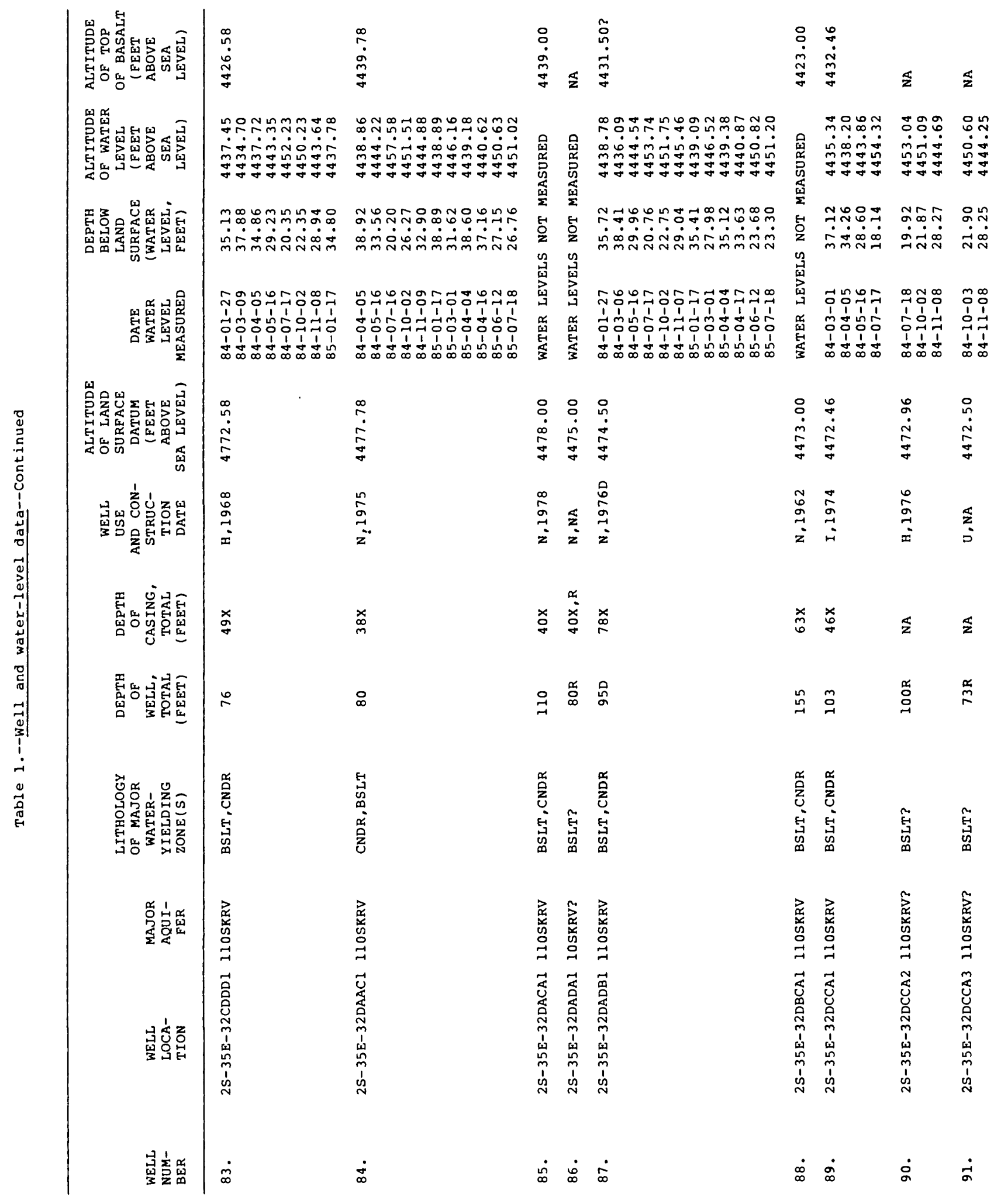




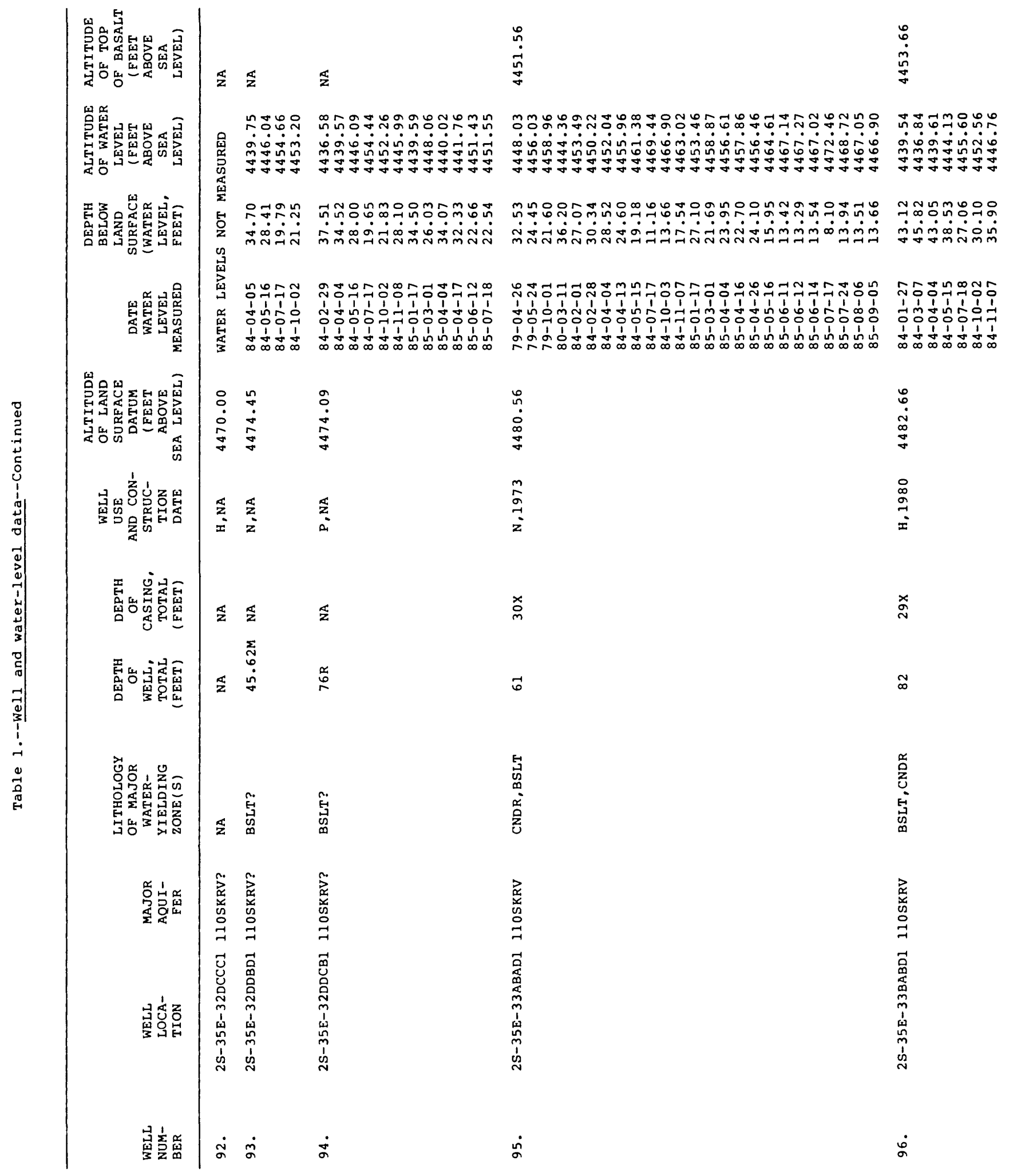




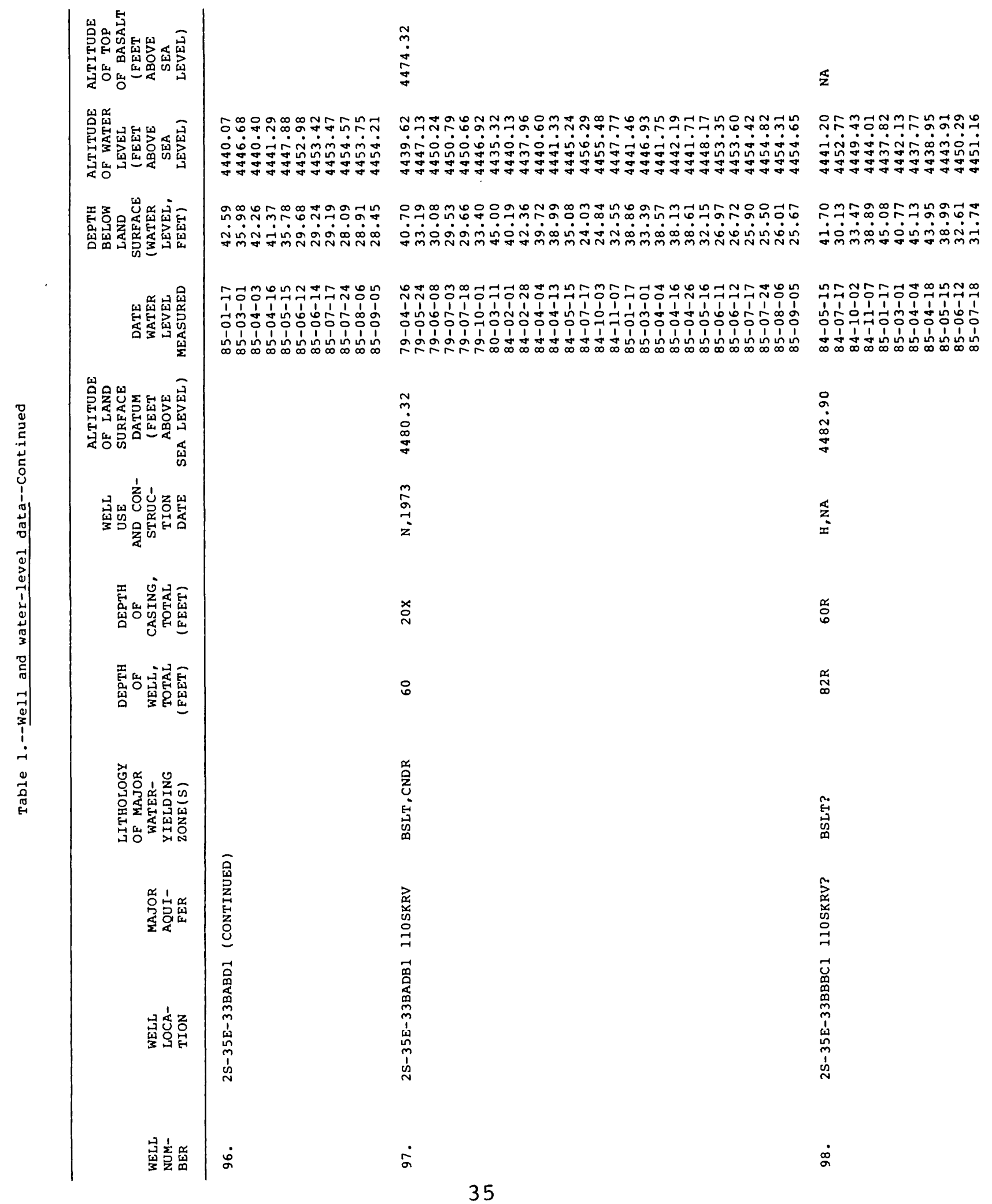




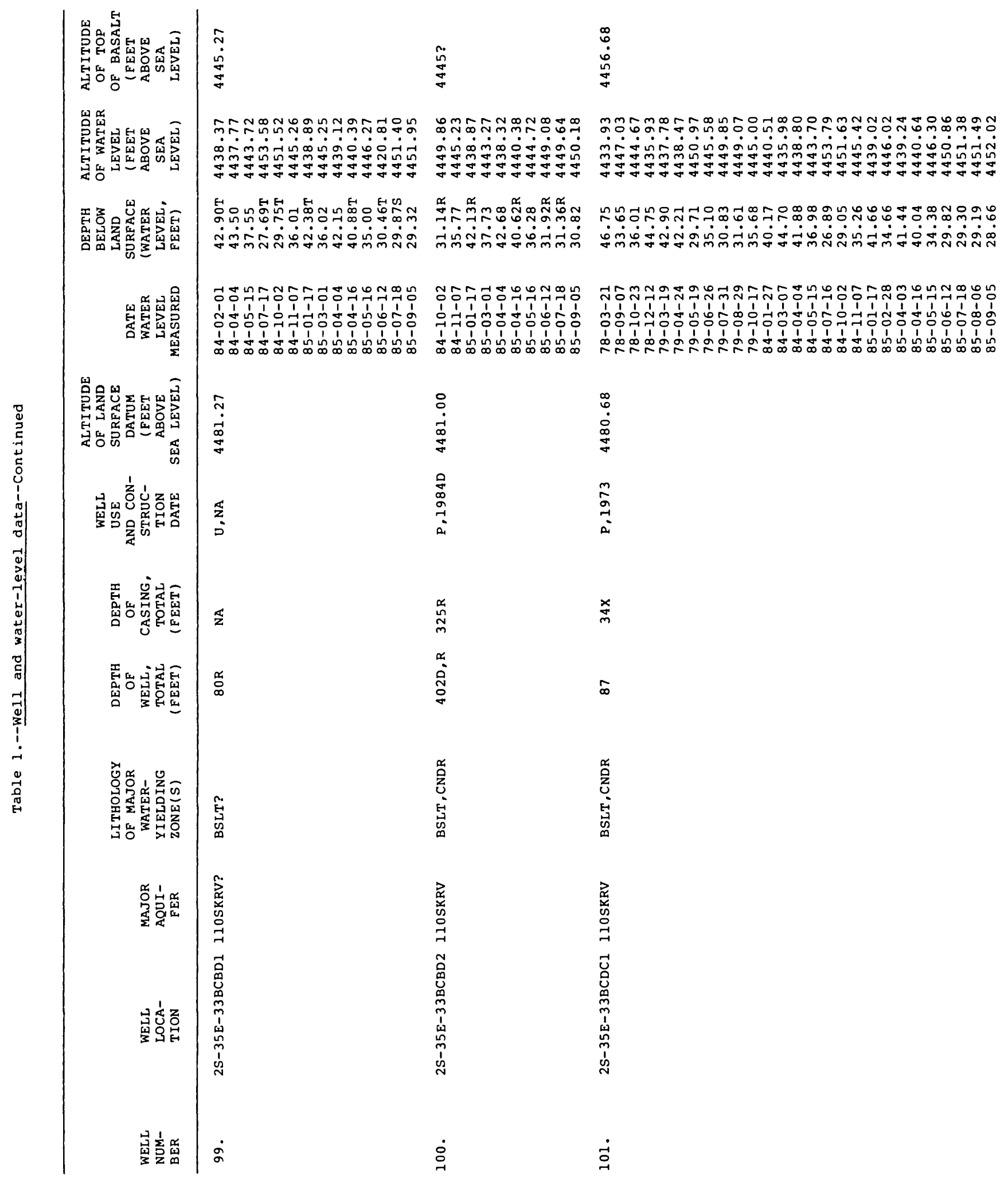




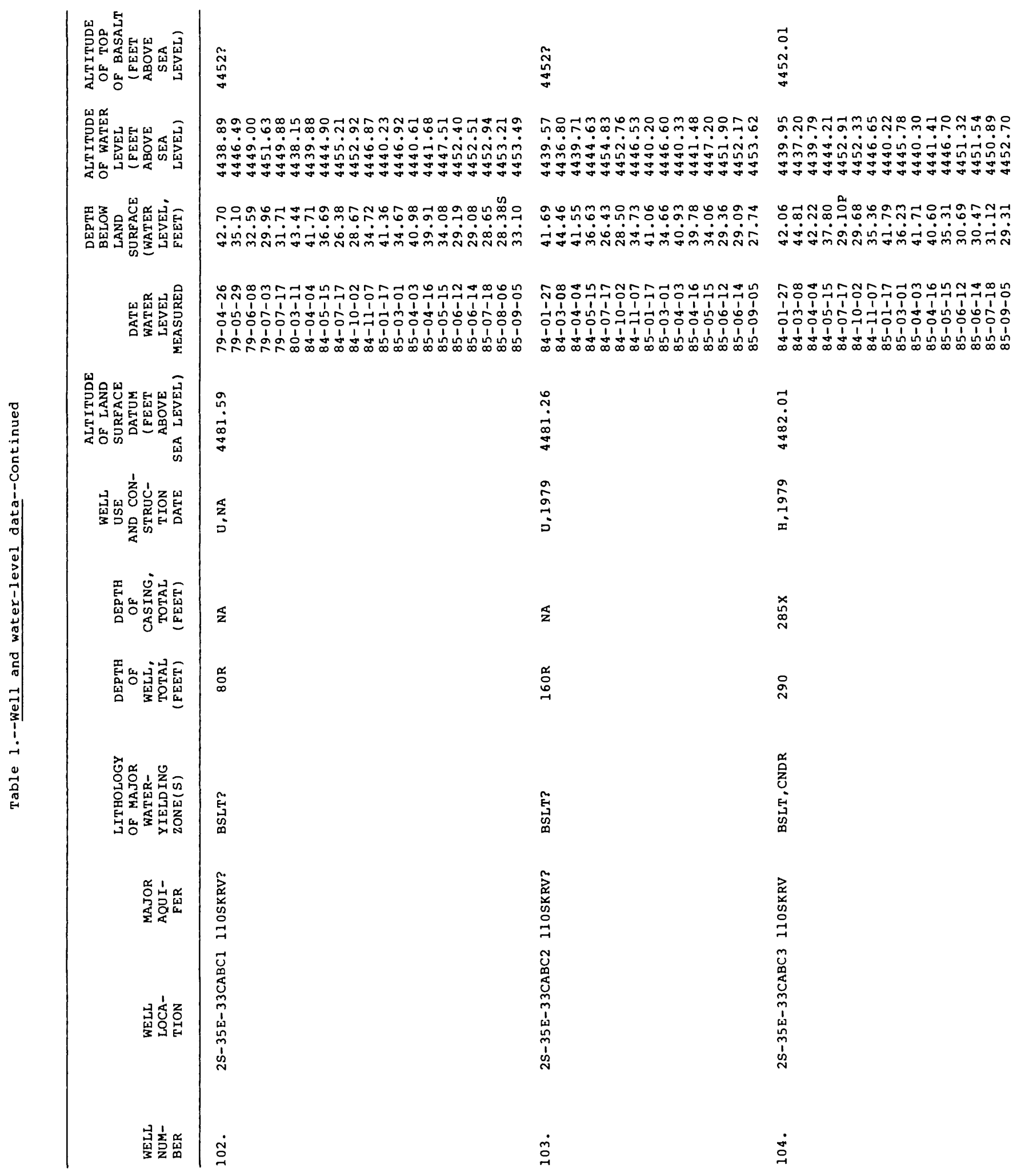




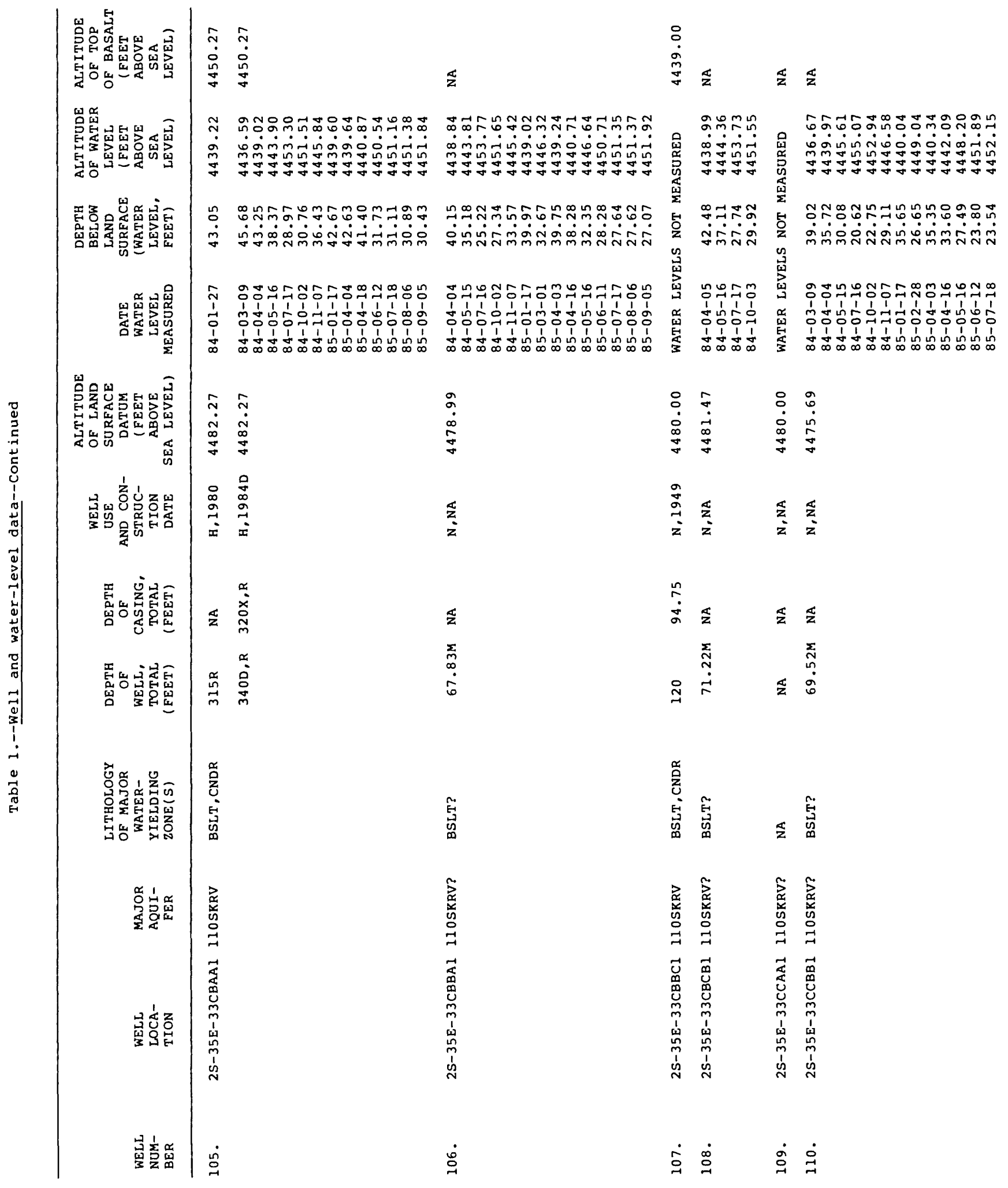




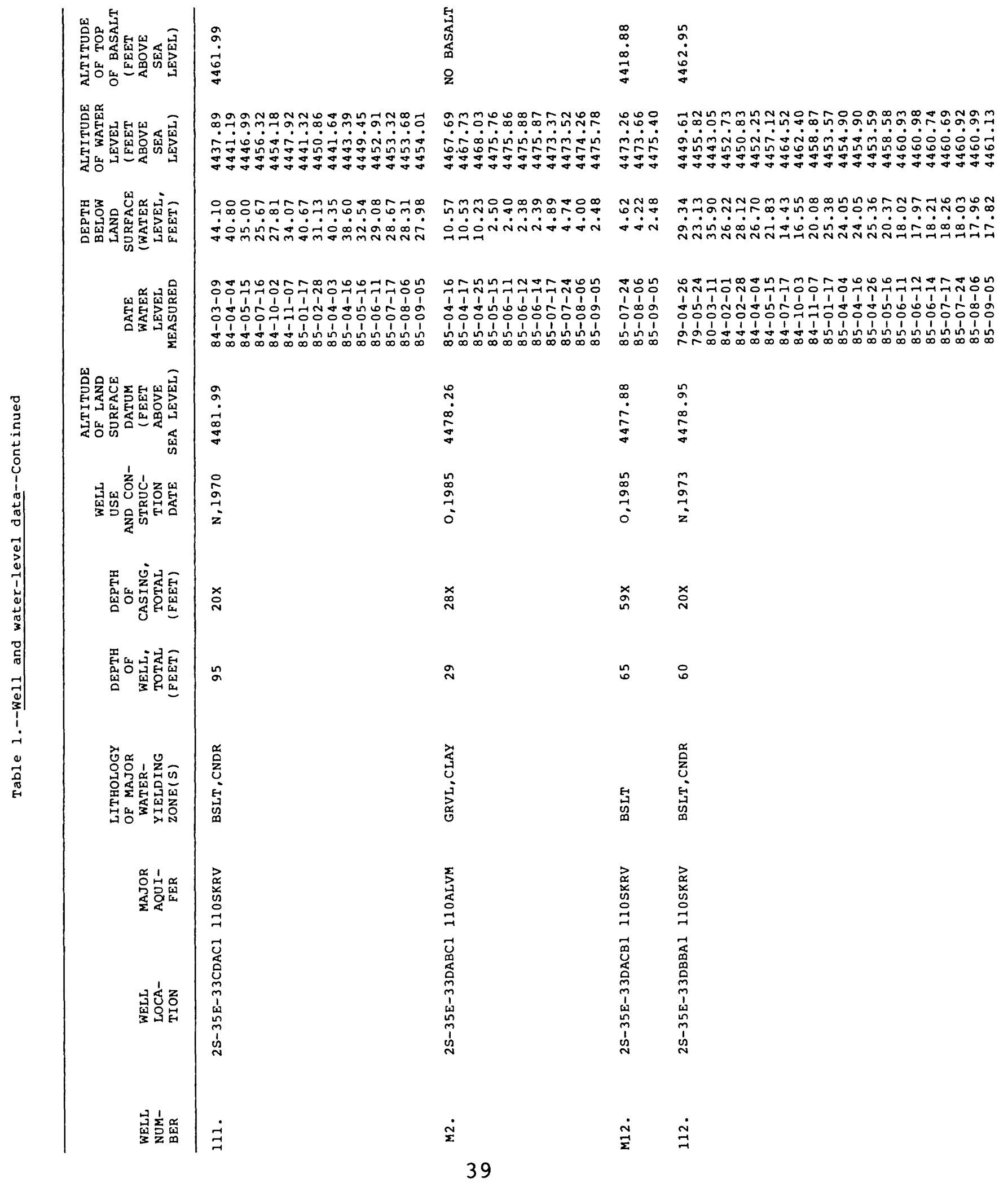




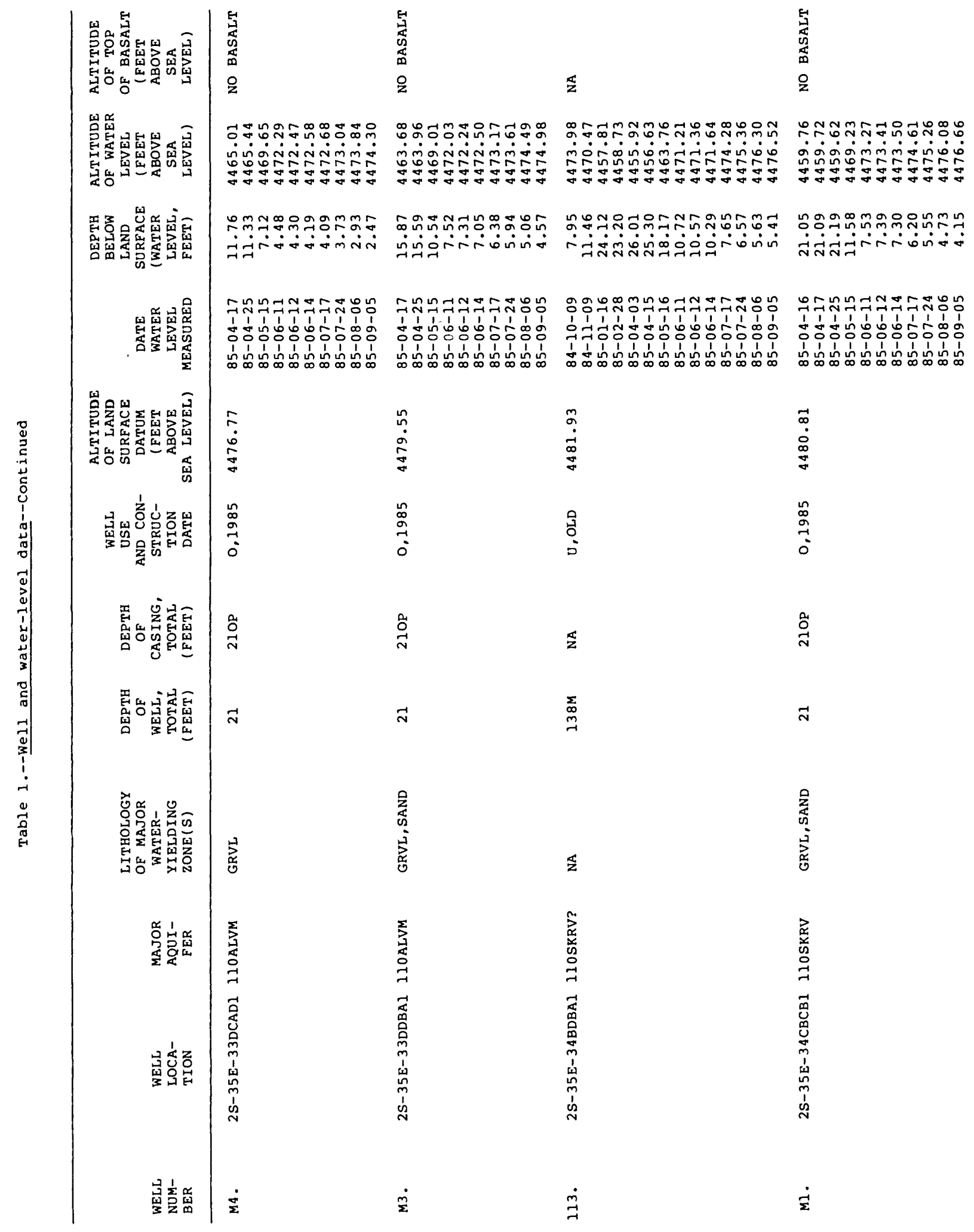




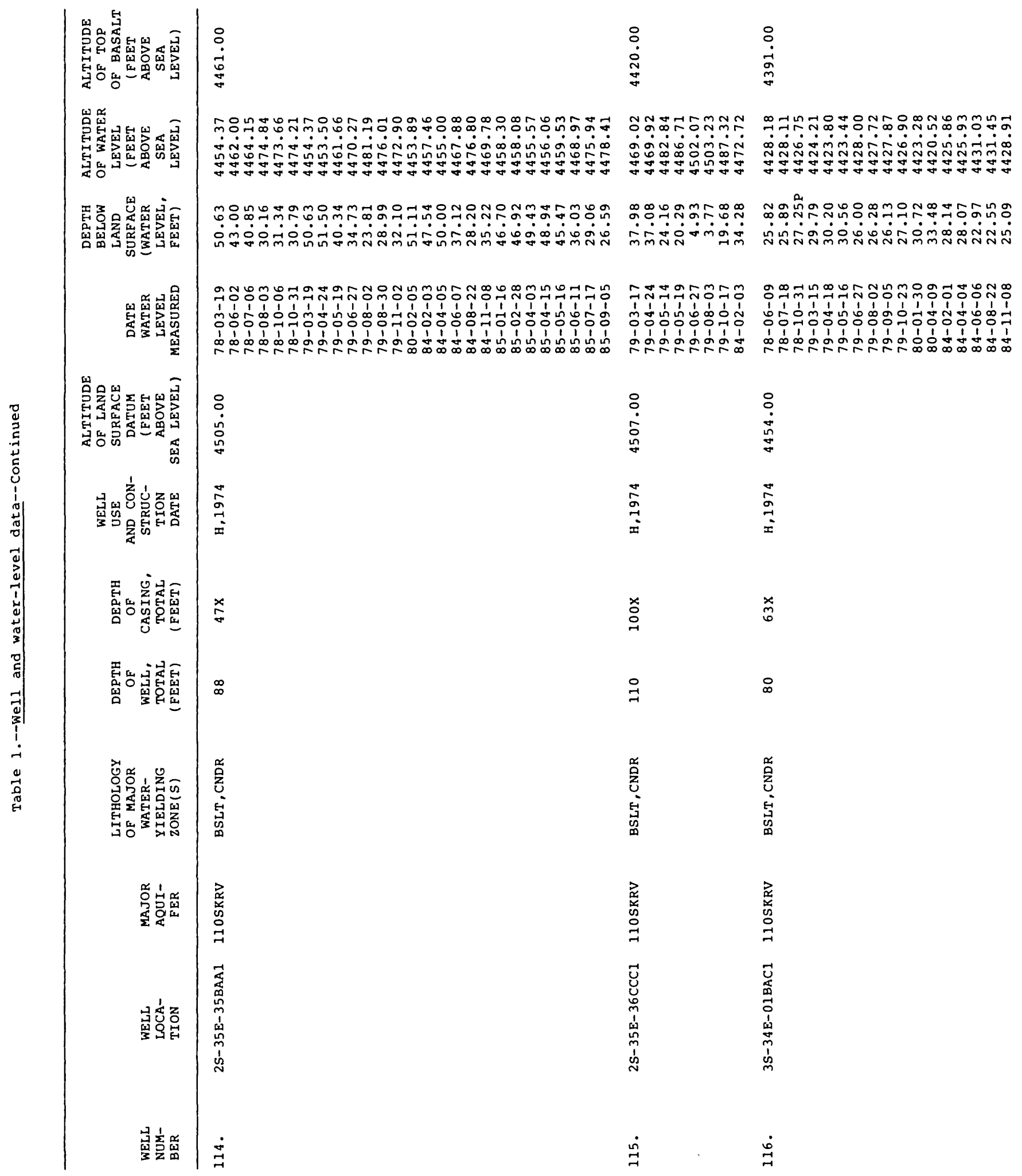




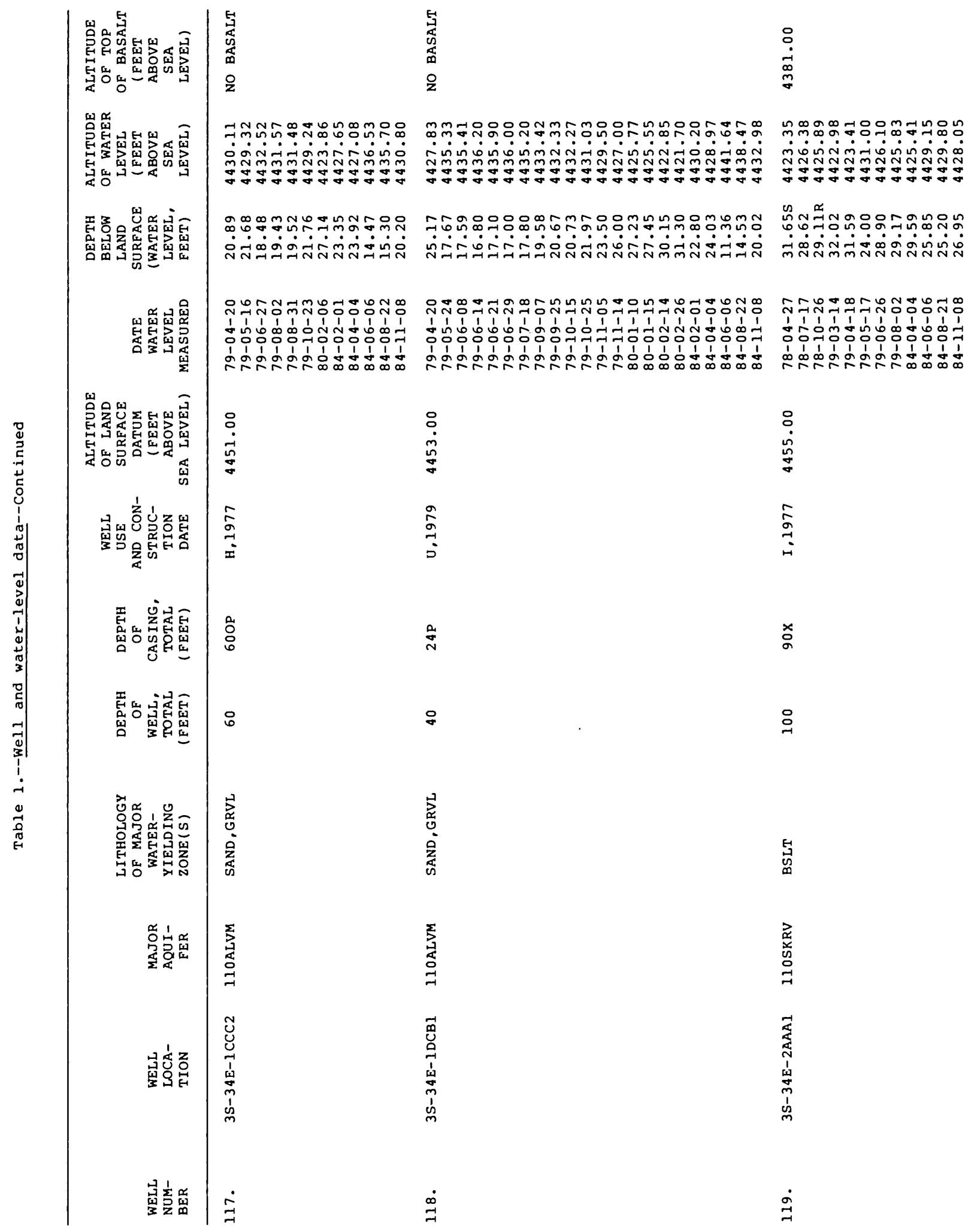




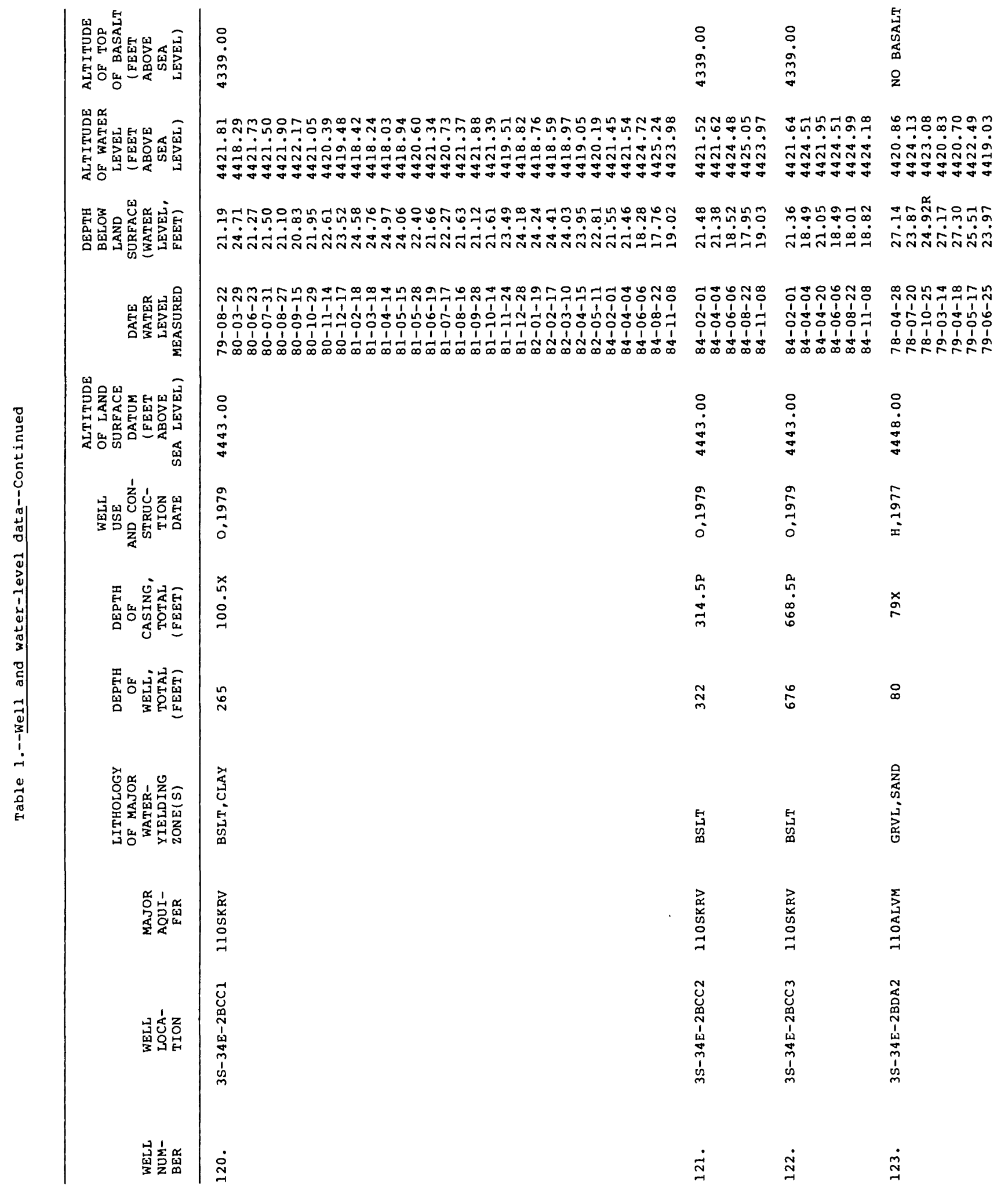




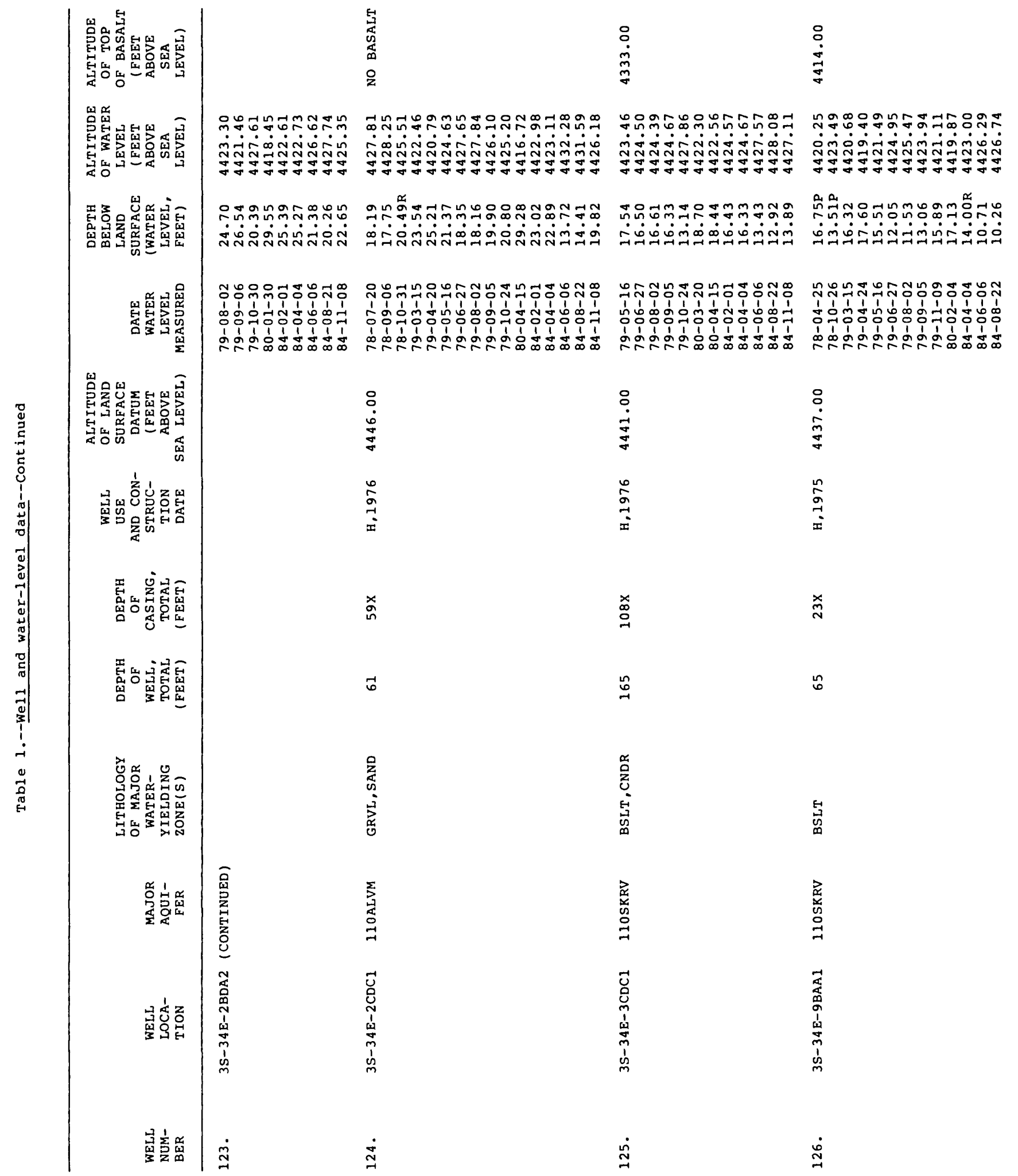




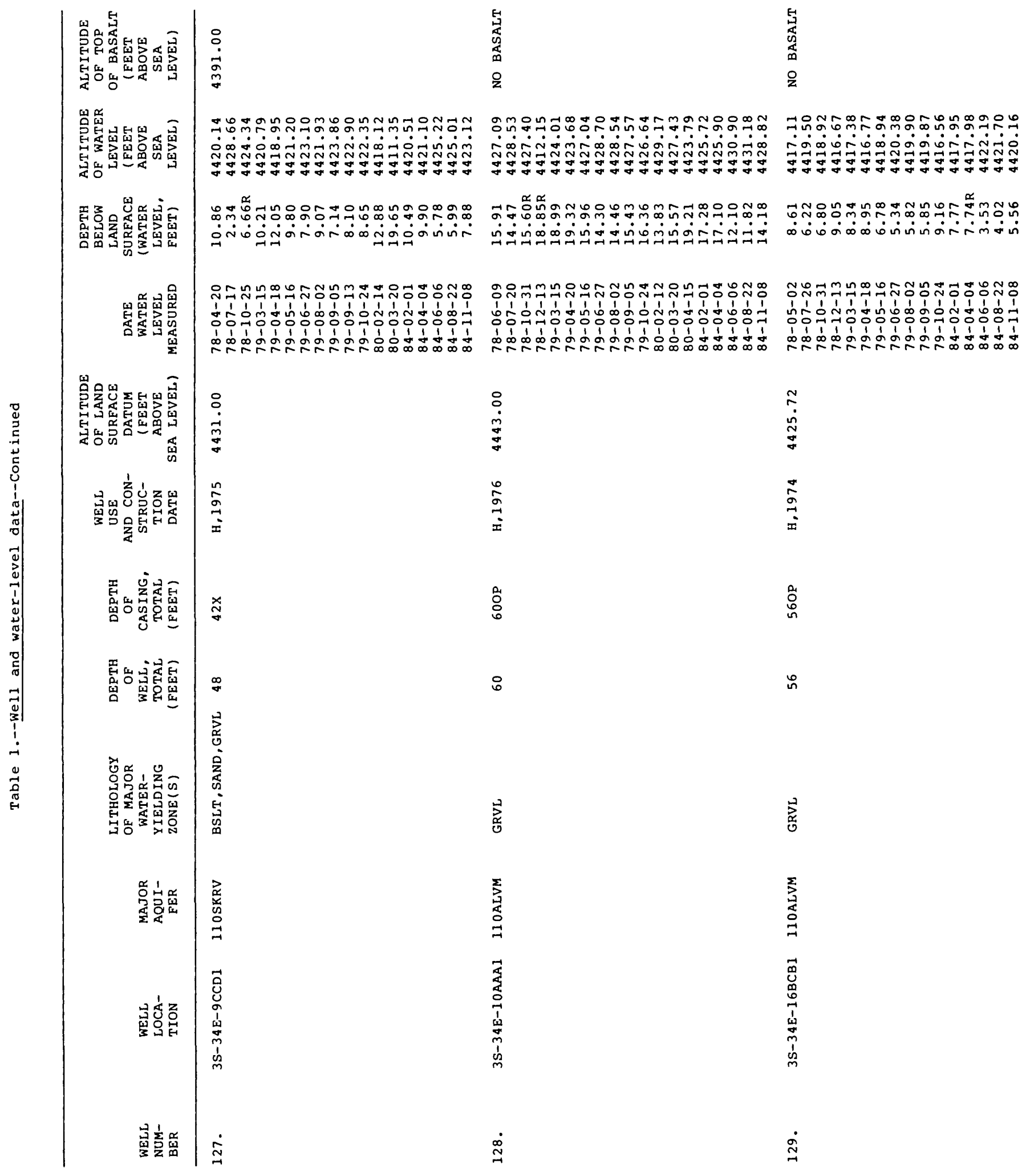




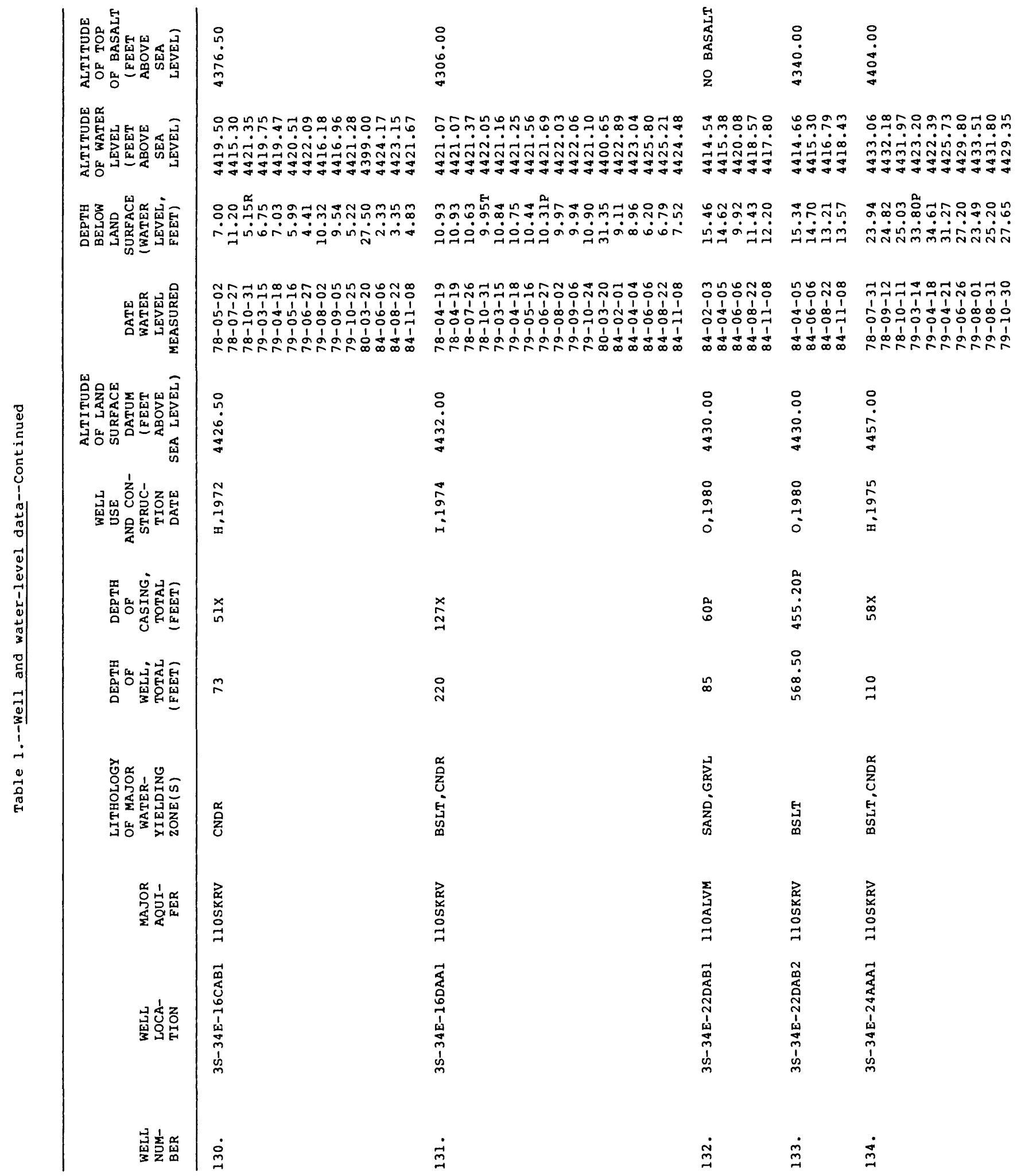




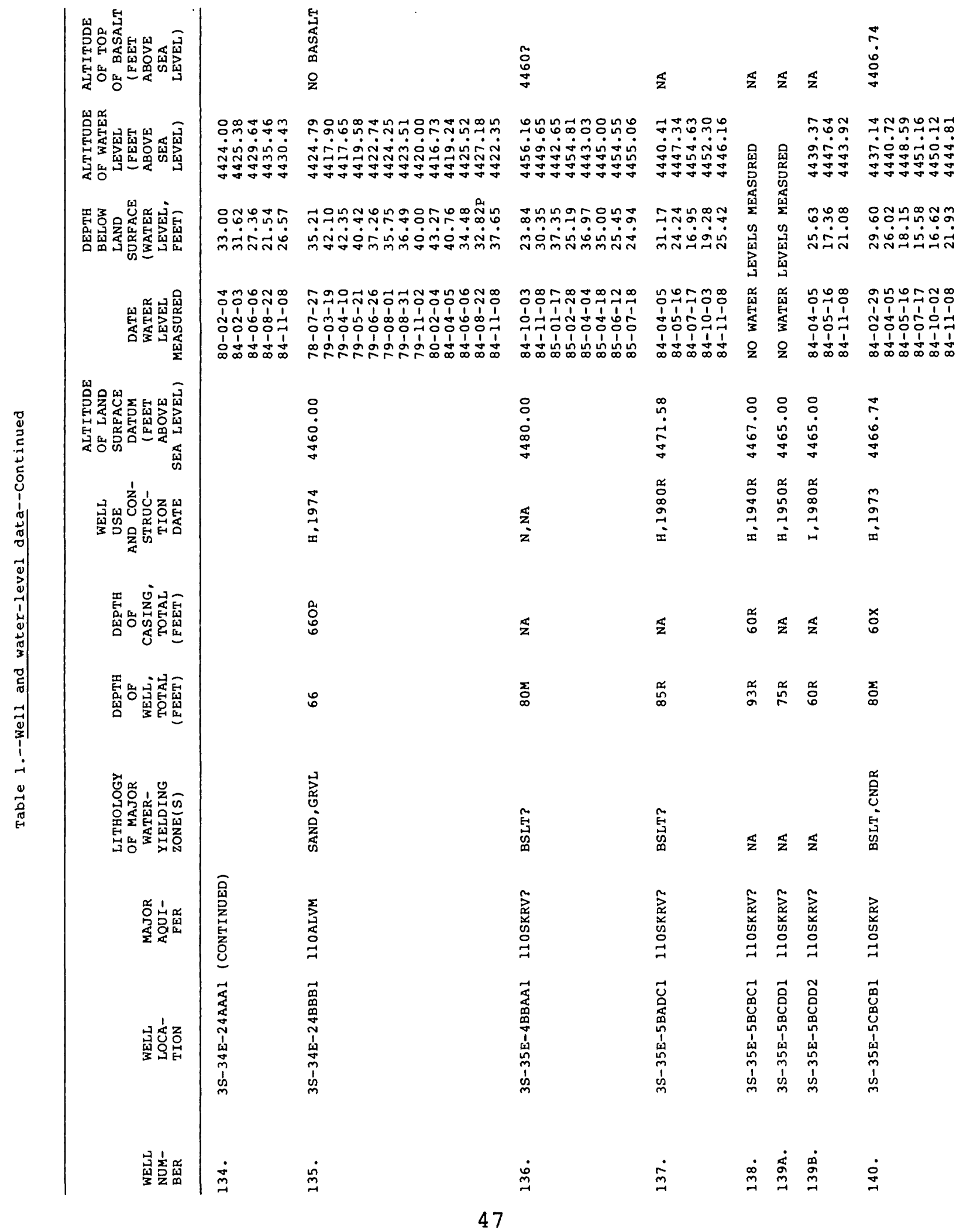




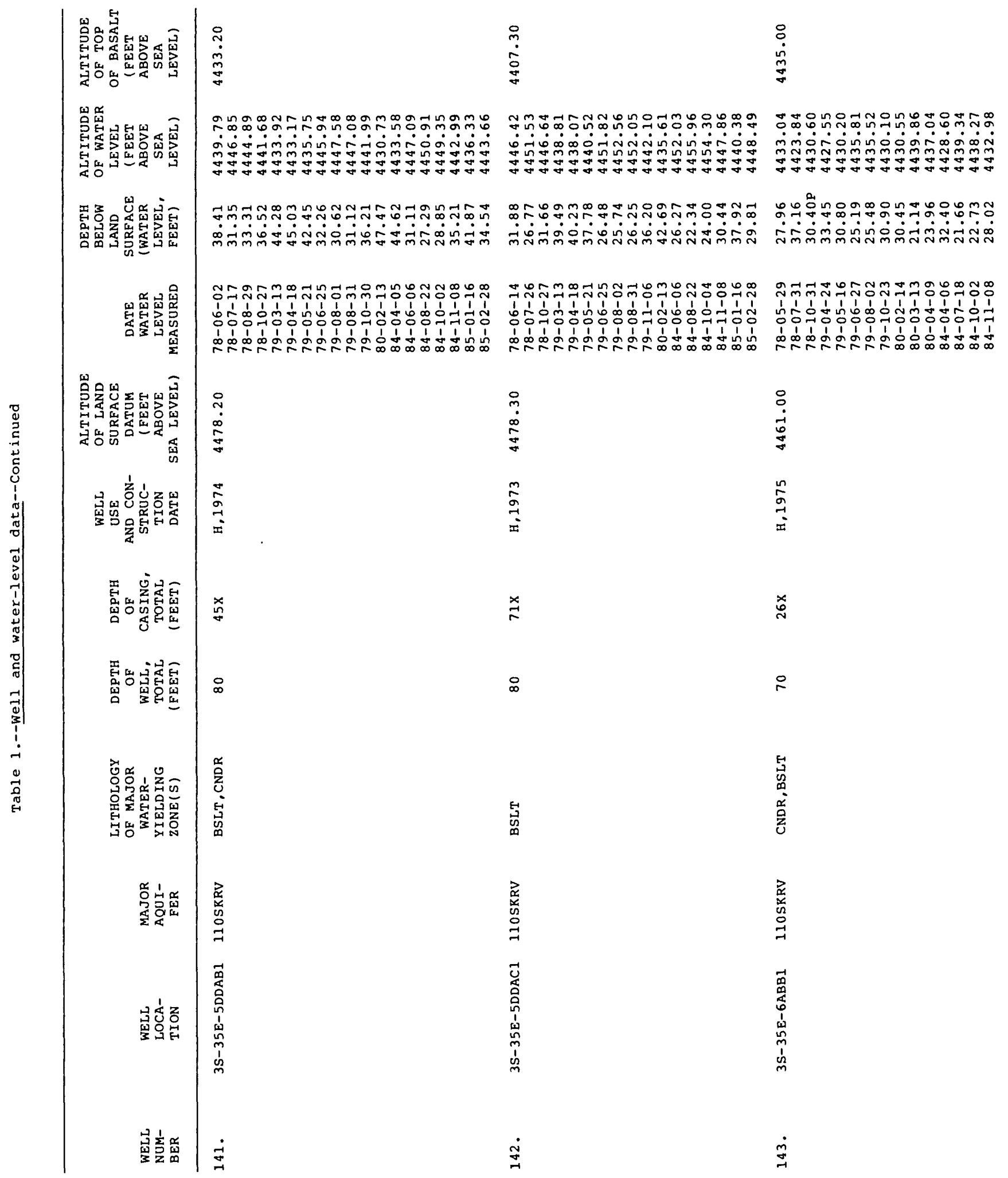




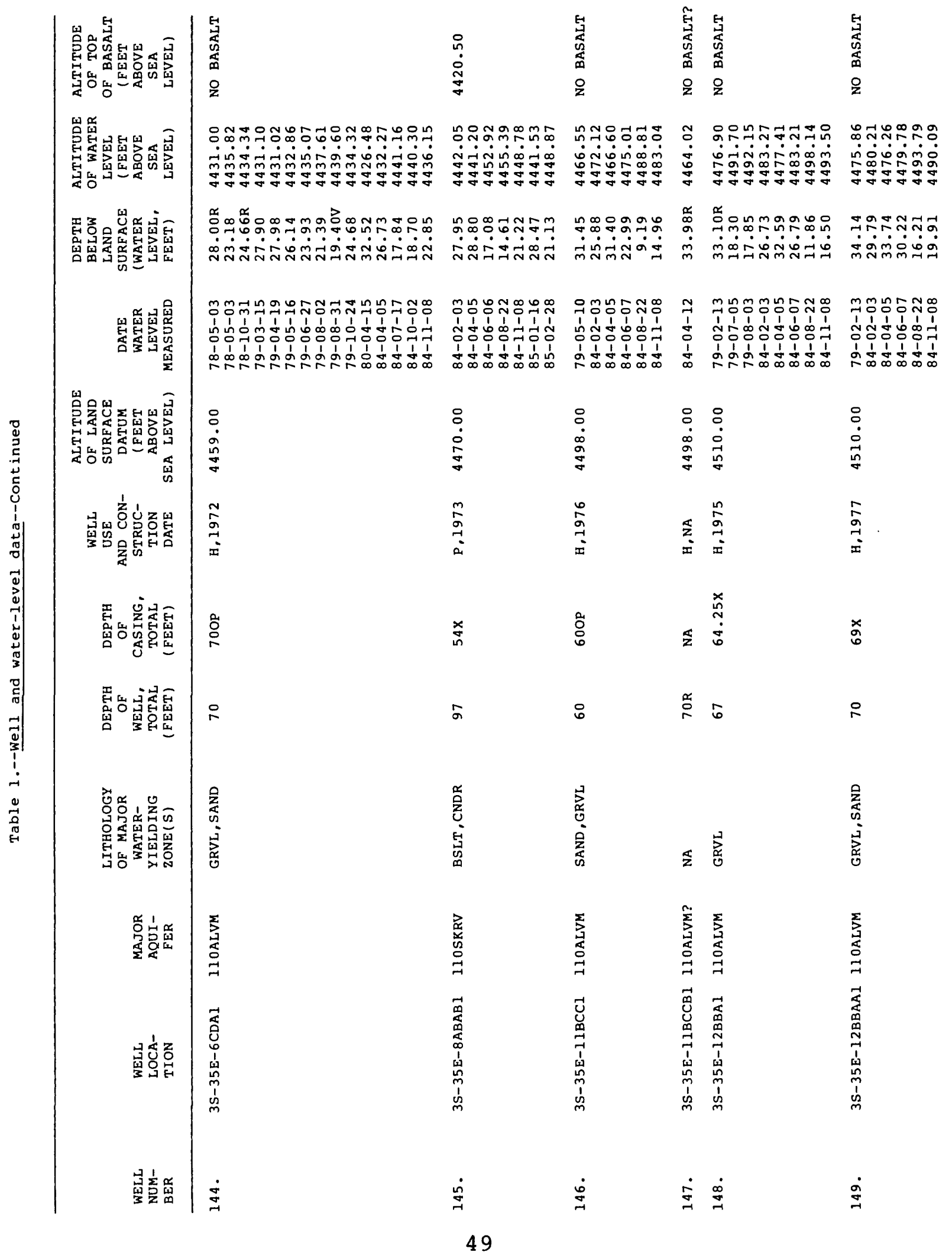




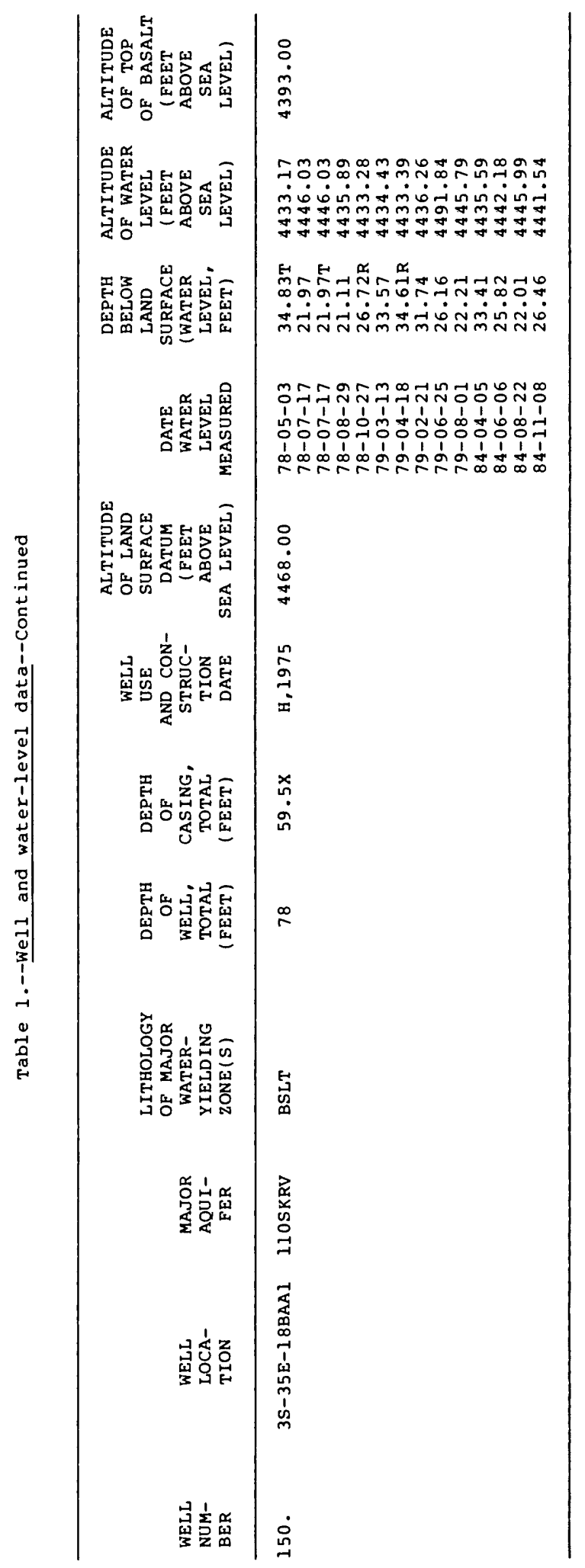


Table 2.--Ground-water quality data, pre-1984

Headnotes

DATE OF SAMPLE: month-day-year

TOTAL WELL DEPTH:

M - measured

$\mathrm{R}$ - reported

COLLECTING AGENCY:

B - Bingham County

E - Idaho Department of Health and Welfare, Division of Environment

GS - U.S. Geological Survey

H - Idaho Department of Health and Welfare, Southeastern District Health Department

Notations:

MG/L - milligrams per liter; within the range of values presented in this report, milligrams are numerically equal to parts per million

COD - chemical oxygen demand

UG/L - micrograms per liter; within the range of values presented in this report, micrograms are numerically equal to parts per billion

$0 \quad$ - analyzed for but not detected

-- - information not available

$<\quad-$ less than

$>\quad$ - greater than

TNTC - too numerous to count 


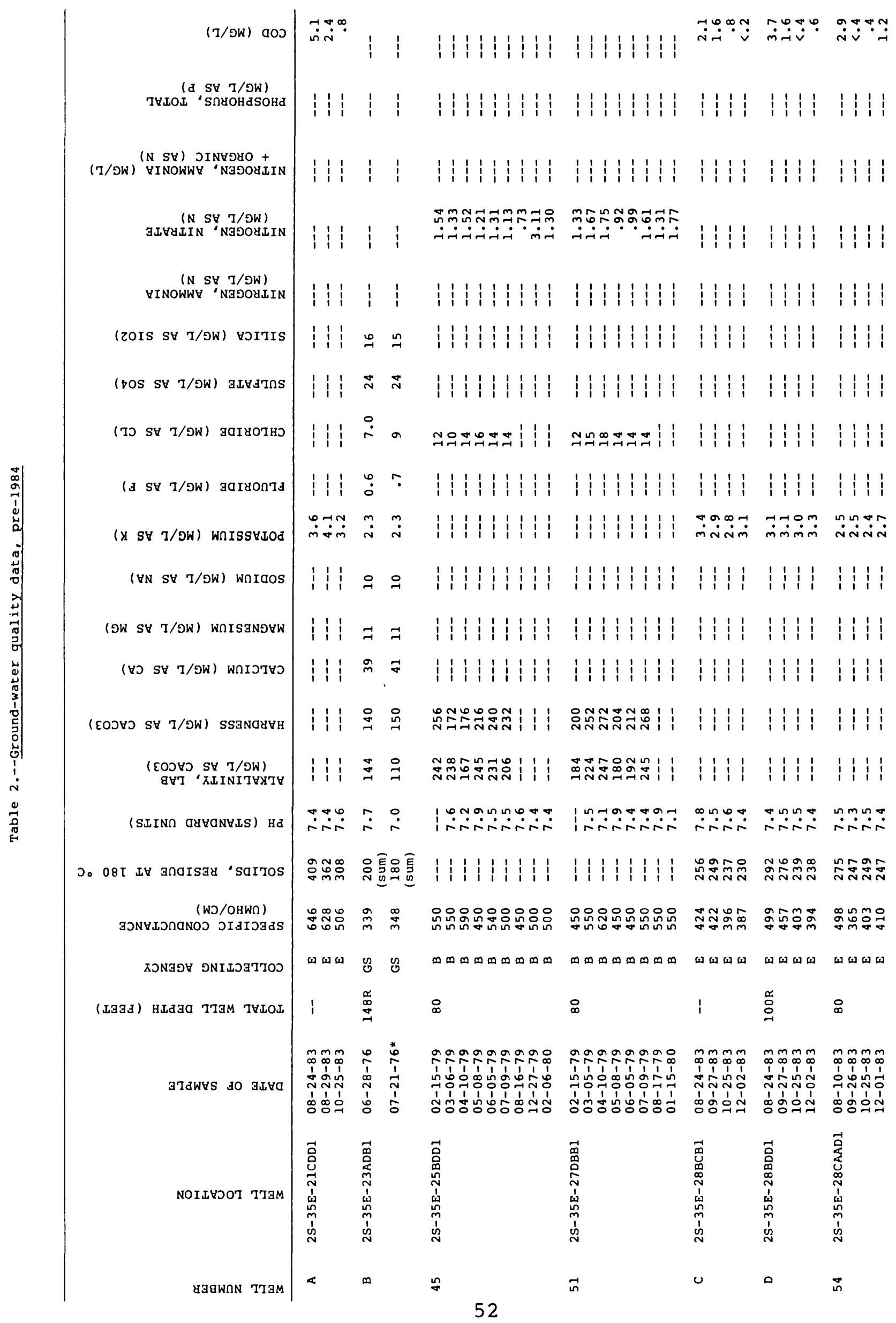




\begin{tabular}{|c|c|c|c|c|c|c|c|}
\hline (NZ I/ON) JNIZ & $\begin{array}{l:l}1 & \vdots\end{array}$ & 1 & 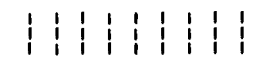 & $\begin{array}{lllll:ll} & 1 & 1 & 1 & 1 & 1 & 1 \\
1 & 1 & 1 & 1 & 1\end{array}$ & $\begin{array}{l:l:l}1 & 1 & 1 \\
1 & 1 & 1\end{array}$ & 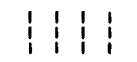 & \\
\hline 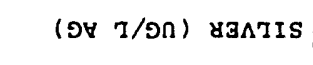 & $\begin{array}{l:l}1 & 1 \\
1 & 1\end{array}$ & 1 & 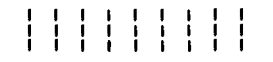 & $\begin{array}{lllllll}1 & 1 & 1 & 1 & 1 & 1 \\
1 & 1 & 1\end{array}$ & $\begin{array}{l:l:l}1 & 1 & 1 \\
1 & 1 & 1\end{array}$ & $1: 11:$ & \\
\hline (3S $t / 9 n$ ) wกINa 13 S & $1: 1$ & $i$ & 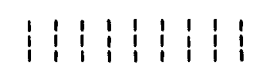 & \begin{tabular}{l|l|l|l|l|l}
1 & 1 & 1 & 1 & 1 & 1 \\
1 & 1 & 1 & 1 & 1
\end{tabular} & $1: 1: 1$ & $\begin{array}{l:ll}1 & 1 & 1\end{array}$ & 1 \\
\hline 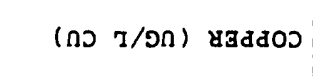 & $\begin{array}{l:l}1 & 1\end{array}$ & $i$ & 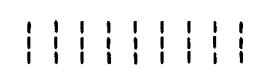 & 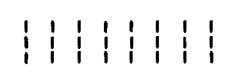 & 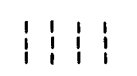 & 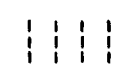 & จร \\
\hline (צכ $T / פ n$ ) wnIwoצks & $1: 1$ & $i$ & $1: 11: 14: 13$ & 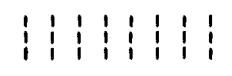 & 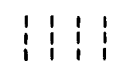 & $\begin{array}{l}1 \\
1\end{array} 1 \vdots$ & \\
\hline (aכ $I / פ n$ ) WnIway & $11 \vdots$ & $i$ & $1: 11: 11: 15: 1$ & 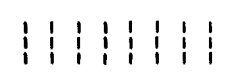 & 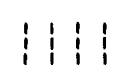 & 1 1111 & \\
\hline (พg $T /$ I ) wกItug & $1: 1$ & $i$ & 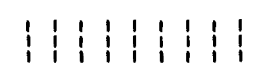 & 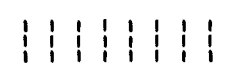 & 1111 & 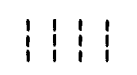 & \\
\hline (SY T/OR) JINGSYY & $1: \vdots$ & $i$ & 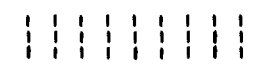 & 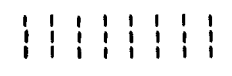 & 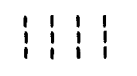 & $\begin{array}{lll}1 & 1 & 1 \\
1 & 1 & 1\end{array}$ & \\
\hline 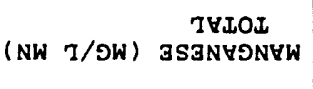 & $1: 1$ & 1 & 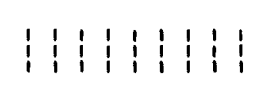 & 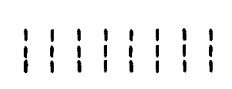 & 1111 & '１：１： & \\
\hline W $T /$ OW) 3S3NYONYW & 운원 & & 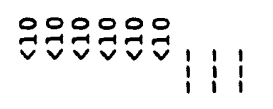 & 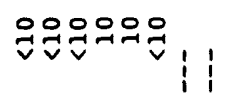 & 욱 & 욱은 & \\
\hline OL (3a T/OW) NOYI & $1: 1$ & 1 & 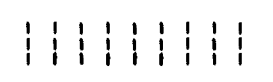 & 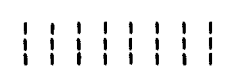 & 1111 & 1１１１１ & \\
\hline (3] $\mathrm{I} / \mathrm{OS})$ NOYI & $\underset{\forall}{\forall}$ & & 육육영 & 요웅유유국 & & & \\
\hline 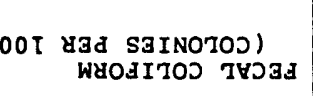 & $1 ! 1$ & 1 & $\nabla \nabla \vec{\nabla} \nabla \mid \vdots ! \vdots$ & $\nabla \nabla \nabla: \vdots 1$ & 111 & 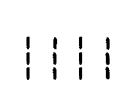 & \\
\hline $\begin{array}{l}\text { OI צ3a SIINOTOJ) } \\
\text { WYOSITOJ THLOL }\end{array}$ & $\vec{v} \vec{v}$ & $!$ & 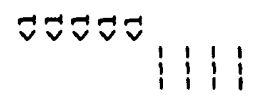 & 2 & $\nabla$ & & \\
\hline J7dWHS so JuYa & 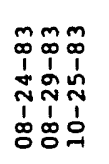 & 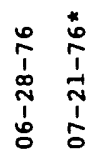 & 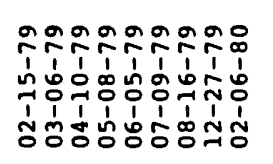 & 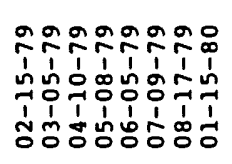 & 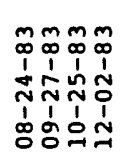 & 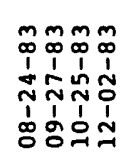 & $\begin{array}{ll}7 \\
1 \\
0 \\
0\end{array}$ \\
\hline NOILYDOT TIJM & 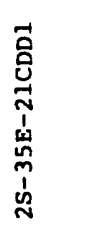 & 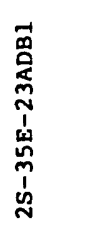 & 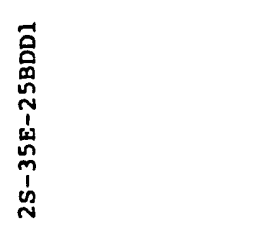 & 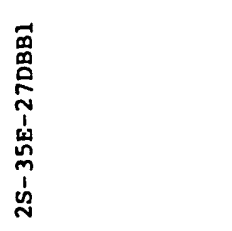 & 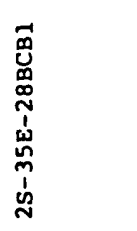 & 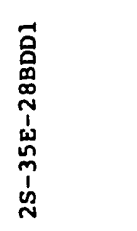 & 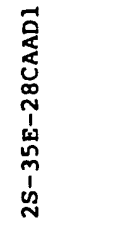 \\
\hline צ3EWลN $773 M$ & $<$ & $\infty$ & & $v$ & & o & ת \\
\hline
\end{tabular}




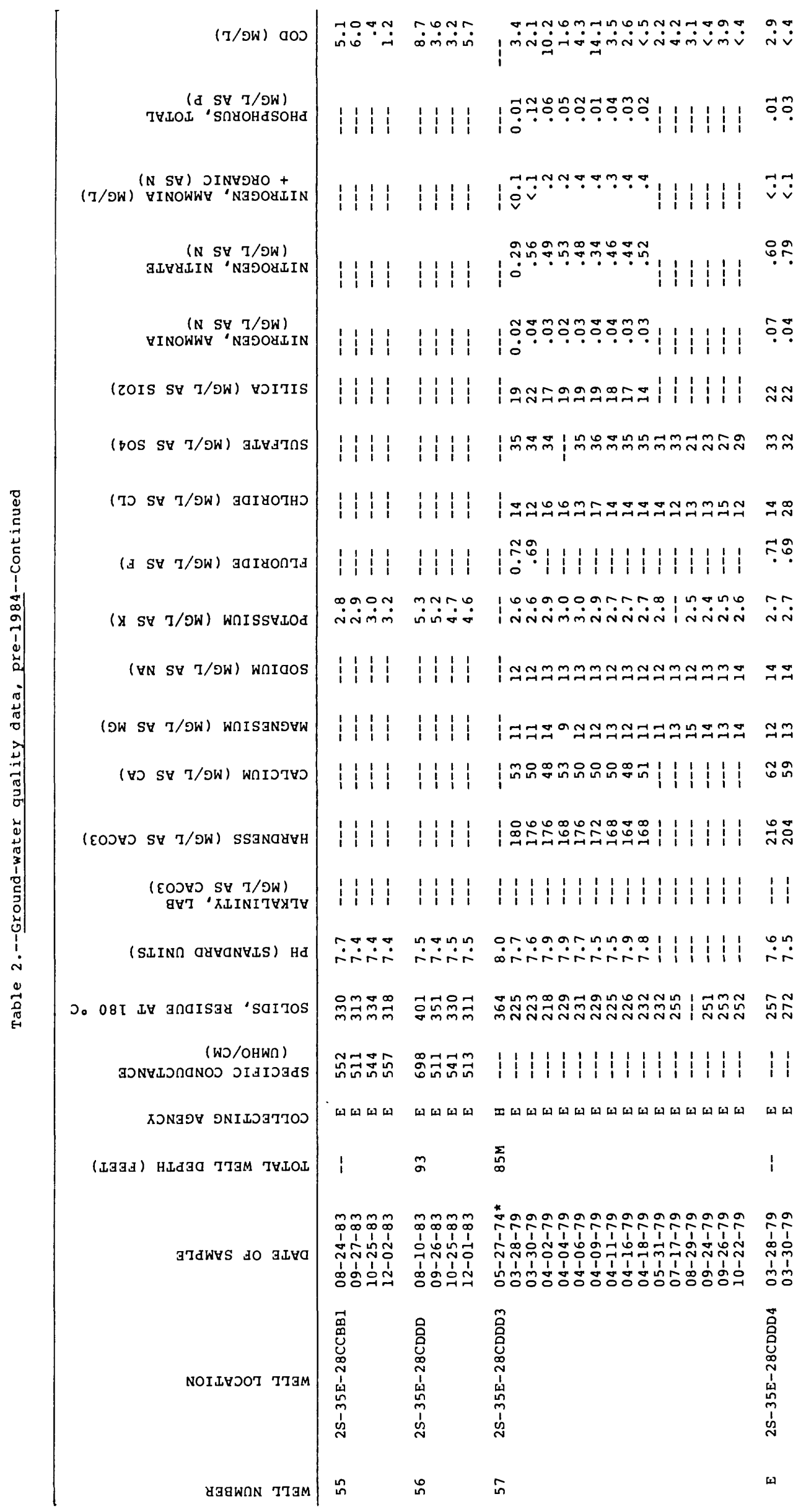




\begin{tabular}{|c|c|c|c|c|}
\hline (NZ $7 / D R$ ) JNIZ & 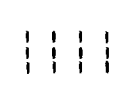 & กิ్ํ: & 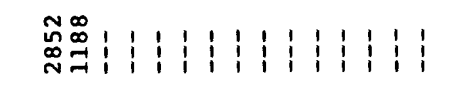 & $\stackrel{\infty}{\infty} \underset{-1}{\infty}$ \\
\hline 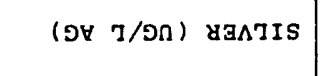 & 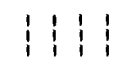 & $\begin{array}{l:ll}1 & 1 & 1 \\
1 & 1\end{array}$ & 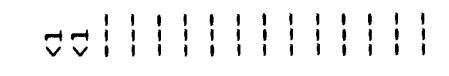 & $\nabla \tau$ \\
\hline (as I/פn) wกINatas & $1: 11:$ & $151: 1$ & ฆ๊ำ: & $\approx v$ \\
\hline ( $\mathrm{I} \supset / \mathrm{J}$ ) y & $1: 111$ & $\stackrel{\sim}{\rightarrow}$ & 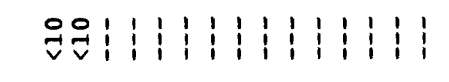 & 요 \\
\hline (צ $7 / 9 n$ ) wnIพоชнอ & $\begin{array}{llll}1 & 1 & 1 & 1 \\
1 & 1 & 1 & 1\end{array}$ & $\stackrel{9}{\nabla} \div$ & 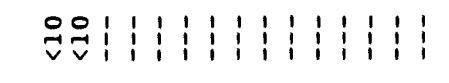 & 5 \\
\hline (ם $\tau / \mathrm{I}$ ) wnIwaษ & 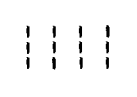 & $\nabla \nabla \vdots \vdots$ & 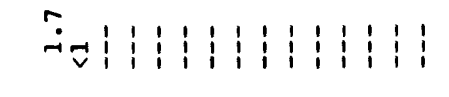 & 5 \\
\hline (ษ8 $7 / 9 n$ ) พกIษษ8 & $\begin{array}{l:ll}1 & 1 & 1 \\
1 & 1 & 1\end{array}$ & $\vdots \vdots$ & 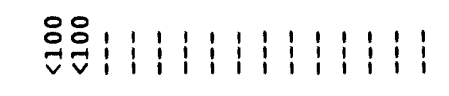 & $\vdots$ \\
\hline (s甘 T/פR) JINGSYY & $\begin{array}{llll}1 & 1 & 1 & 1 \\
1 & 1 & 1\end{array}$ & $1: 1: 1$ & 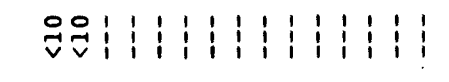 & 우웅 \\
\hline W I/OW) $\begin{array}{c}\text { IษLOI } \\
\text { ISINYONYW }\end{array}$ & 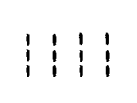 & & 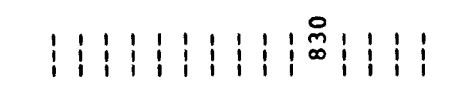 & \\
\hline (W I/OW) ISINYDNYW & 운워 & 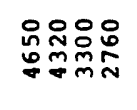 & 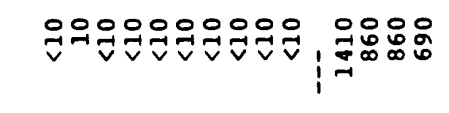 & \\
\hline OL (GS I/JW) NO\&I & $\begin{array}{l:ll}1 & 1 & 1\end{array}$ & $i$ & 141 & \\
\hline (3A $7 / 9 n$ ) NOYI & 운원워 & :요 & 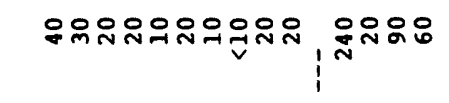 & \\
\hline 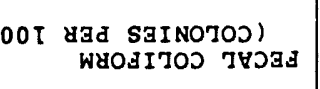 & $1: 11$ & $1 \vdots$ & 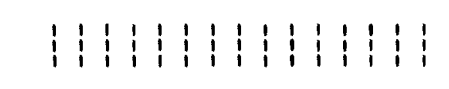 & 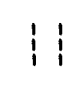 \\
\hline 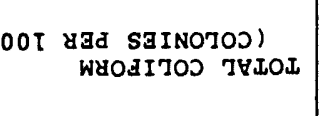 & $\vec{v}+\vec{v} \vec{v}$ & $i i^{\circ}$ & 1 & \\
\hline Jjawys so $3 u \forall d$ & 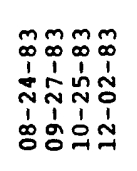 & 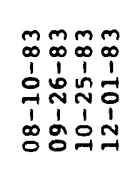 & 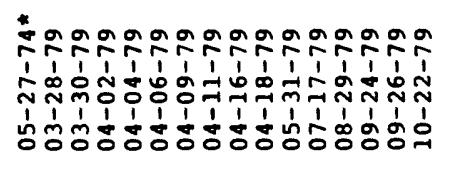 & 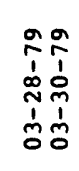 \\
\hline NOILYSOI IJaM & 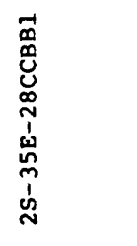 & 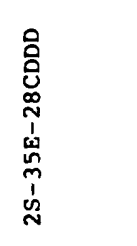 & 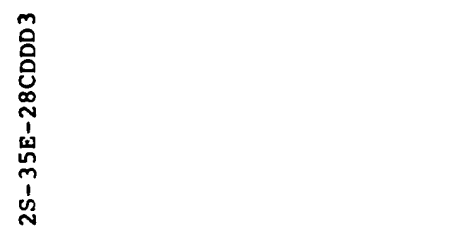 & 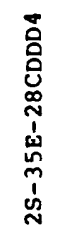 \\
\hline ४ $38 \mathrm{QWN}$ TI3M & in & $\sigma$ & in & \\
\hline
\end{tabular}




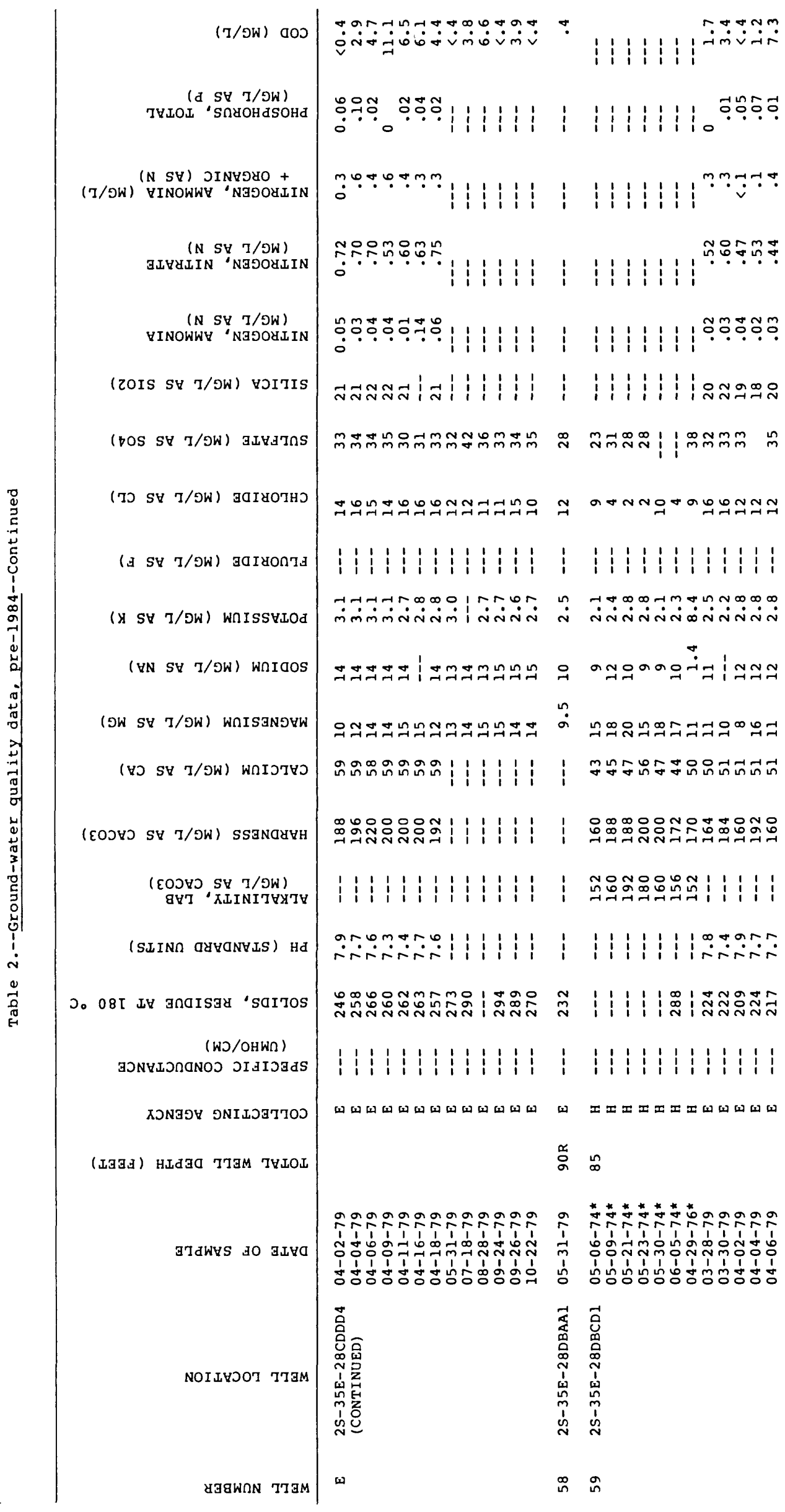




\begin{tabular}{|c|c|c|}
\hline (NZ T/OR) JNIZ & 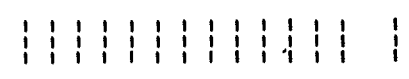 & 1:1:1: $\left.\right|^{\infty}$ \\
\hline 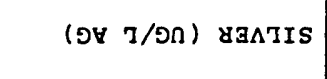 & 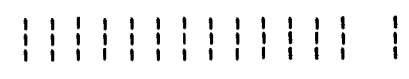 & $1: 15: 1: 5 \approx 1$ \\
\hline (as $7 / O n$ ) wnINGTas & 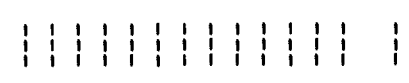 & 1:1:1:1: 1 \\
\hline 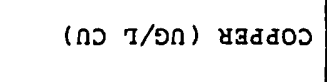 & 1:1:1:1:1:1:1:1: & 1:11:1:告 \\
\hline (ष $7 / פ \cap)$ พกIพоษнว & 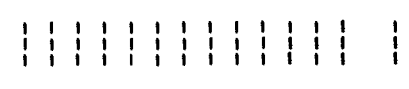 & 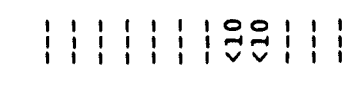 \\
\hline (a) $I / D n$ ) WกIways & 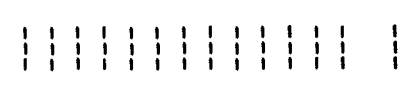 & $\begin{array}{lll:l:l}1 & 1 & 1 & 1 & \vdots\end{array}$ \\
\hline (ษg $7 / O \cap)$ พกIษษ & 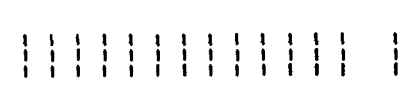 & 1H11: \\
\hline (SY J/OR) JINaSYY & 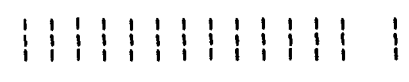 & 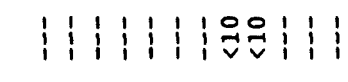 \\
\hline (NW T/OW) aSaNHONYW & 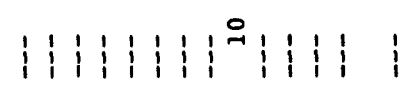 & $1: 1: 15: 1$ \\
\hline (NW T/OW) aSaNYSNYW & 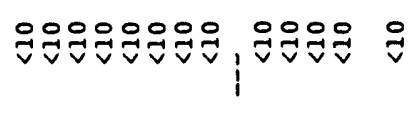 & 1111: \\
\hline IHLOI ( $\mathrm{GA} \mathrm{I} / \mathrm{OW})$ NOYI & 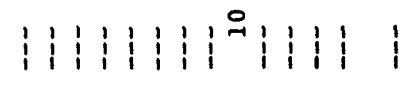 & 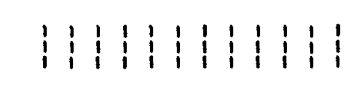 \\
\hline$(3 A T / O n)$ NOAI & 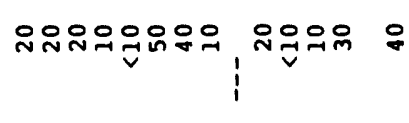 & 1 \\
\hline $\begin{array}{l}\text { Wroditos thosa } \\
00 t \text { yad SaINOTOJ) }\end{array}$ & 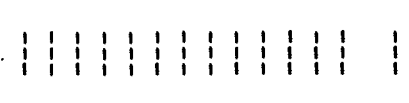 & $11: 11: 1^{2} 1: 1$ \\
\hline $\begin{array}{l}\text { OOT Yad SAINOTOJ) } \\
\text { WHOSITOJ THLOI }\end{array}$ & 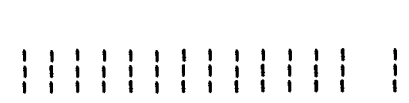 & $\approx \approx 1: 1 i^{\approx}$ \\
\hline 37 dWYS so 3iYG & 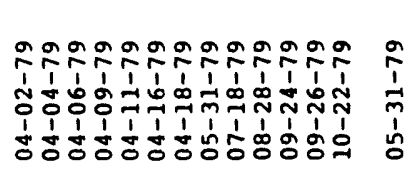 & 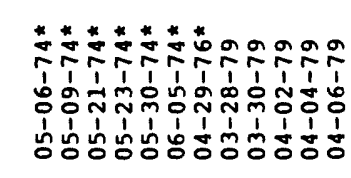 \\
\hline NOILYJOT TIJM & 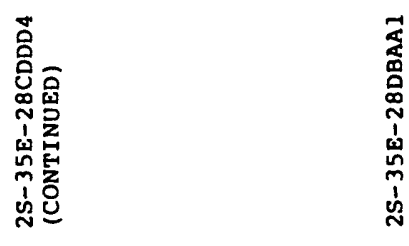 & 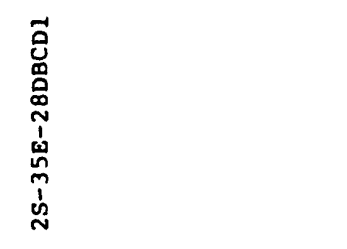 \\
\hline 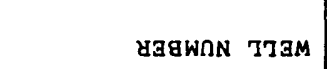 & นั & in \\
\hline
\end{tabular}




\begin{tabular}{|c|c|c|c|c|c|}
\hline$(\mathrm{T} / \mathrm{OW})$ aos & 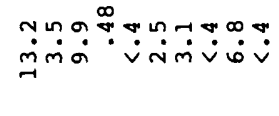 & $\begin{array}{l:l:l}1 & 1 & 1 \\
1 & 1 & 1\end{array}$ & $1: 1: 1$ & 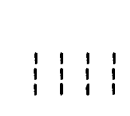 & 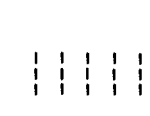 \\
\hline 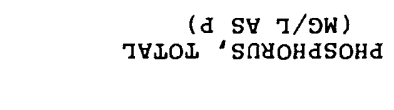 & 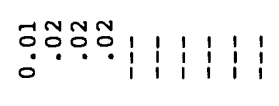 & 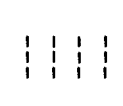 & $\begin{array}{ll:l}1 & 1 & \vdots \\
1 & 1 & 1\end{array}$ & $\begin{array}{l:ll}1 & 1 & \vdots \\
1 & 1\end{array}$ & $\begin{array}{l:l}1 & \vdots \\
1 & 1\end{array}$ \\
\hline 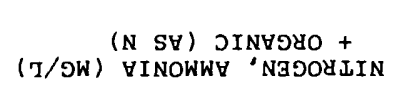 & 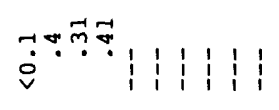 & $\begin{array}{lll}1 & 1 & 1 \\
1 & 1\end{array}$ & 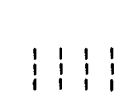 & $\begin{array}{ll:l}1 & 1 & 1 \\
1 & 1 & 1\end{array}$ & $1: 1$ \\
\hline $\begin{array}{c}(\mathrm{N} S \mathrm{TH} T / \mathrm{TW}) \\
\text { ALYYLIN }\end{array}$ & 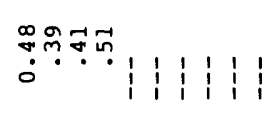 & 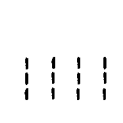 & 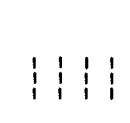 & $\begin{array}{ll:l}1 & 1 & 1 \\
1 & 1 & 1\end{array}$ & 191 \\
\hline $\begin{array}{c}\text { (N SH T/OW) } \\
\text { YINOWWY "NGDOYLIN }\end{array}$ & 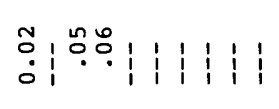 & 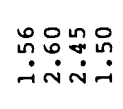 & 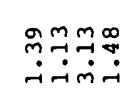 & 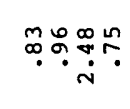 & $\stackrel{m}{m} \underset{\infty}{\infty}: r$ \\
\hline (ZOIS SH T/OW) צDITIS & 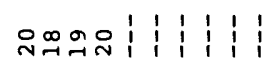 & 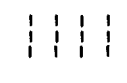 & $\begin{array}{llll}1 & 1 & 1 & \vdots \\
1 & 1 & 1\end{array}$ & $\begin{array}{llll}1 & 1 & 1 & 1\end{array}$ & 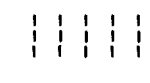 \\
\hline 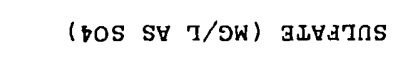 & దำกี & $\begin{array}{ll:l}1 & 1 & 1 \\
1 & 1 & 1\end{array}$ & 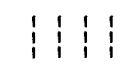 & $\begin{array}{llll}1 & 1 & 1 & 1 \\
1 & 1 & 1\end{array}$ & $1: 1$ \\
\hline 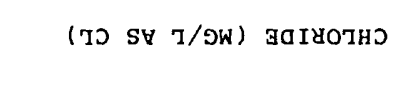 & 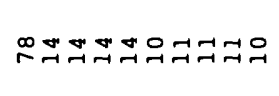 & $\begin{array}{llll}1 & 1 & 1 & \vdots \\
1 & 1 & 1\end{array}$ & $\curvearrowleft \begin{array}{l:ll}\curvearrowleft & 1 & 1\end{array}$ & $\approx \begin{array}{l:lll} & & 1 & 1\end{array}$ & 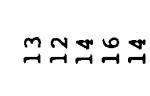 \\
\hline (a SY T/SW) garyonTa & 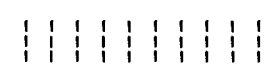 & 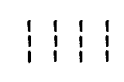 & $\begin{array}{ll:l}1 & 1 & 1\end{array}$ & $\begin{array}{l:l:l}1 & 1 & 1\end{array}$ & $\begin{array}{l:ll}1 & 1 & 1 \\
1 & 1\end{array}$ \\
\hline (x SY T/OW) WnISSYLOd & 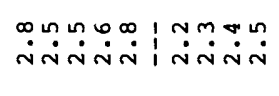 & $\begin{array}{l:ll}1 & 1 & 1 \\
1 & 1 & 1\end{array}$ & $1 !$ & 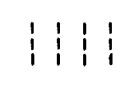 & i \\
\hline ( $\mathrm{YN} \mathrm{SY} T / \mathrm{T}$ ) wחraos & 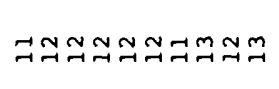 & 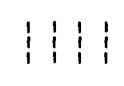 & $1: 1: 1$ & 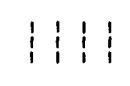 & $\begin{array}{l:l}1 & \vdots \\
1 & 1\end{array}$ \\
\hline ( อW SY T/OW) WกISรNอYW & 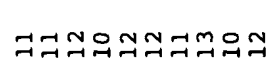 & $\begin{array}{lllll}1 & 1 & 1 & 1 \\
1 & 1 & 1 & 1\end{array}$ & 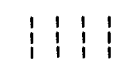 & $1: 11 \vdots$ & 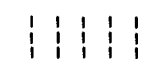 \\
\hline (ษ SY I/פW) พกโวדช & 우ㅇㅛㅡำ: & $1 \vdots 11$ & $\begin{array}{l:ll}1 & 1 & 1\end{array}$ & $1: 11 \vdots$ & $\vdots$ \\
\hline 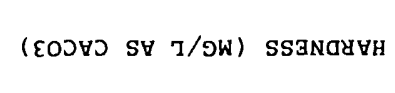 & 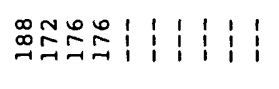 & \begin{tabular}{l:lll}
\multirow{2}{*}{} & 1 & 1 & 1
\end{tabular} & $\begin{array}{l:lll}\tilde{N} & 1 & \vdots\end{array}$ & $\underset{\sim}{\approx}: \vdots \vdots$ & สำํํำ \\
\hline 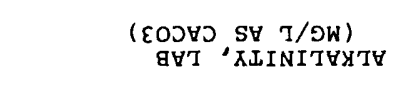 & 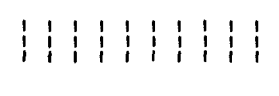 & $\stackrel{9}{\beth}: 11$ & $\begin{array}{c:c:c}m & 0 & \vdots\end{array}$ & 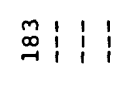 & 馬: \\
\hline (SIIN GYYGNHLS) Hd & 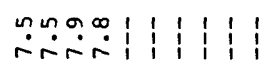 & 至 & 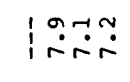 & 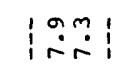 & 10 \\
\hline D. $08 t$ it anarsay ‘sartos & 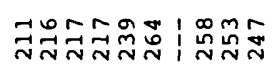 & 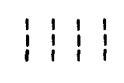 & $1: 11$ & $1: 11$ & $1: 1$ \\
\hline 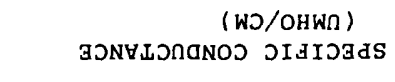 & $\begin{array}{llllllllll}1 & 1 & 1 & 1 & 1 & 1 & 1 & 1 & 1 & 1 \\
1 & 1 & 1 & 1 & 1 & 1 & 1 & 1 & 1\end{array}$ & 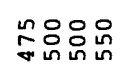 & 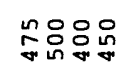 & 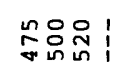 & 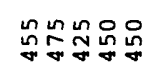 \\
\hline XכNGOY ONIโכJTTOD & 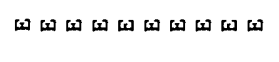 & $m \infty \infty \infty$ & $m \infty \dot{\infty}$ & $m \infty \dot{m}$ & $\infty$ \\
\hline 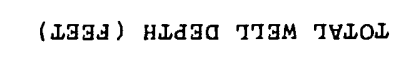 & & 1 & 政 & $\stackrel{n}{\circ}$ & $\stackrel{n}{\sim}$ \\
\hline 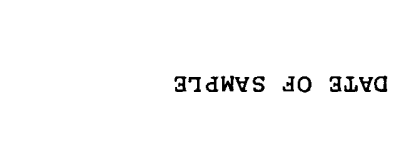 & 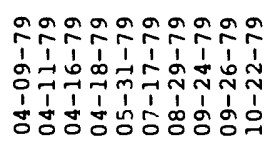 & 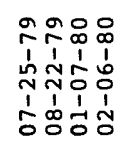 & 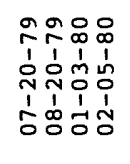 & 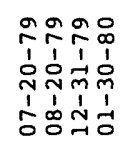 & 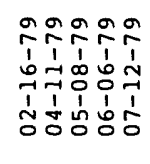 \\
\hline NOILUDOT TIతM & 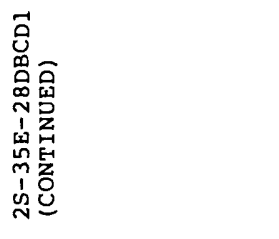 & 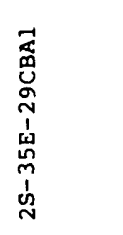 & 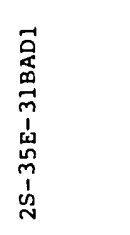 & 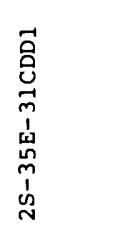 & 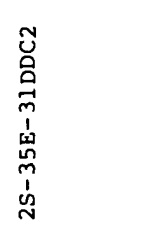 \\
\hline 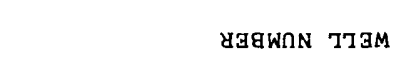 & in & an & 0 & $x$ & $H$ \\
\hline
\end{tabular}




\begin{tabular}{|c|c|c|c|c|c|}
\hline (NZ $\mathrm{T} / \mathrm{ON}$ ) JNIZ & 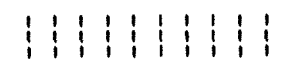 & $1: 1: 1$ & $1: 1 \vdots$ & $1: 1 \vdots 1$ & \\
\hline 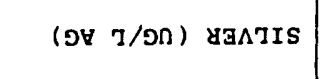 & $1: 1 \vdots 11: 1 \vdots \vdots$ & $1: 11:$ & $1: 1: 1$ & $\begin{array}{l:l}1 & 1 \\
1 & 1\end{array}$ & 1 \\
\hline (3S $7 / 0 n$ ) wกInaras & $1: 11: 11: 1:$ & $\vdots \vdots$ & $1: 1: \vdots$ & 111 & $:$ \\
\hline (nכ $7 / 0 n)$ yaddos & 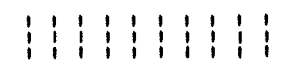 & $1 \vdots$ & $\begin{array}{llll}1 & 1 & 1 & 1\end{array}$ & 1111 & \\
\hline (ष $] / 9 n$ ) wnIwoצH & 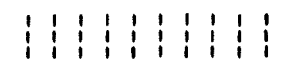 & $i !$ & $1: 1: 1$ & 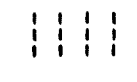 & \\
\hline (बد $7 / פ \cap$ ) พกIพaษ & 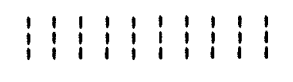 & $1 !$ & 11 & $\begin{array}{llll}1 & 1 & 1 & 1\end{array}$ & \\
\hline (48 $\mathrm{t} / \mathrm{On}$ ) worytg & $1: 11: 11: 11:$ & $i$ & $1: 1$ & 111 & 1 \\
\hline (SH T/Oم) JINJSYY & $1: 11: 11: 1:$ & 1111 & 1111 & $111 i$ & $!$ \\
\hline งW T/OW) $\underset{T \text { ISINYIT }}{\text { ISINYW }}$ & 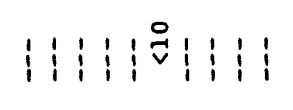 & $1: 1: \vdots$ & $1: 1: 1$ & 1111 & $i$ \\
\hline NW I/OW) 3SGNYDNYW & 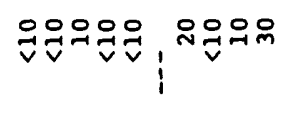 & 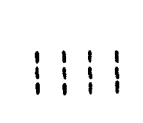 & $1: \vdots$ & $4 \vdots \vdots \vdots$ & \\
\hline IOI (3a $\mathrm{J} / \mathrm{OW}$ ) NOYI & $111 i^{\circ} 11 !$ & 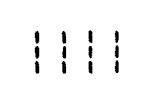 & $\begin{array}{l:}1 \\
1\end{array}$ & $1: 19$ & $i$ \\
\hline (3a $\mathrm{J} / \mathrm{On})$ NO\&I & 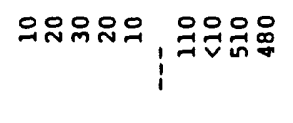 & & & 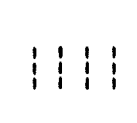 & \\
\hline 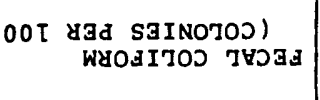 & 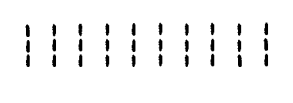 & $1: 11:$ & $1 ! 1 !$ & ! ! ! & \\
\hline $\begin{array}{l}00 T \text { Y3a SaINOTOJ) } \\
\text { WYOAIJOD TYIOI }\end{array}$ & 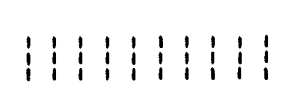 & $1: 1:$ & 1! ! & $\begin{array}{lll}1 & 1 & 1 \\
1 & 1 & 1\end{array}$ & \\
\hline 37dWYS so aJYo & 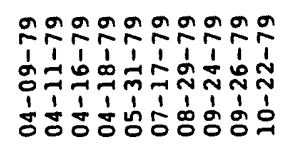 & 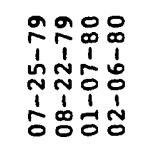 & 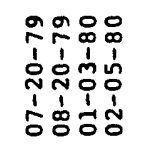 & 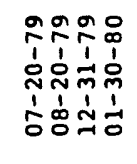 & 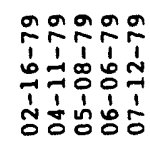 \\
\hline NOIJ甘SOT IJ & 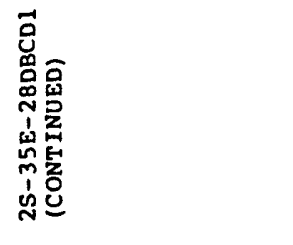 & 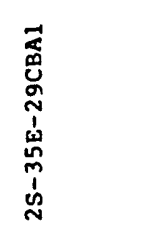 & 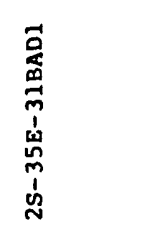 & 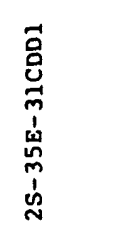 & 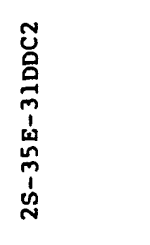 \\
\hline 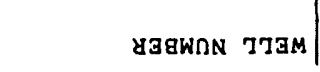 & s & 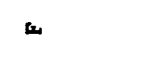 & 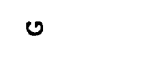 & $=$ & $r$ \\
\hline
\end{tabular}




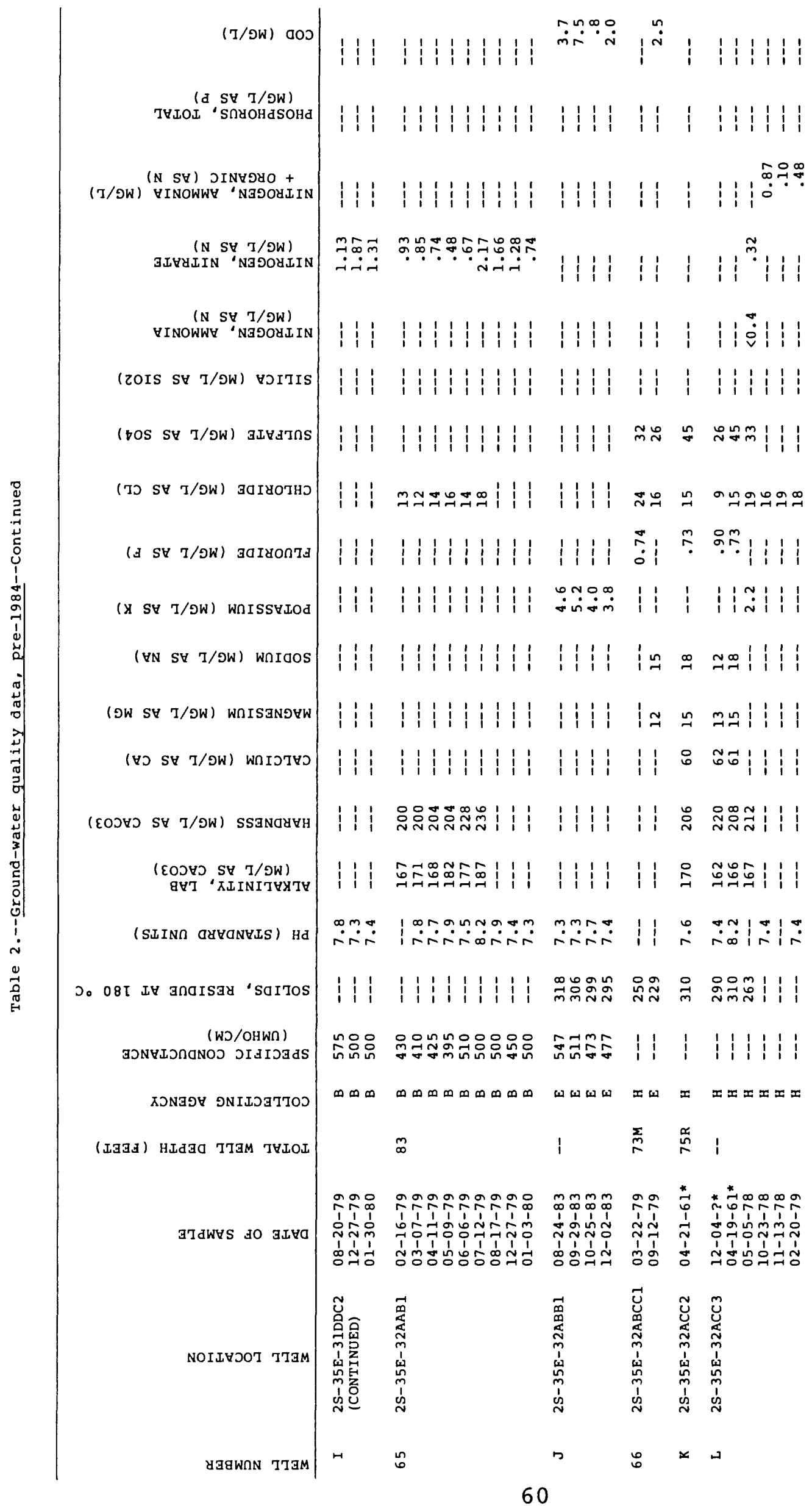




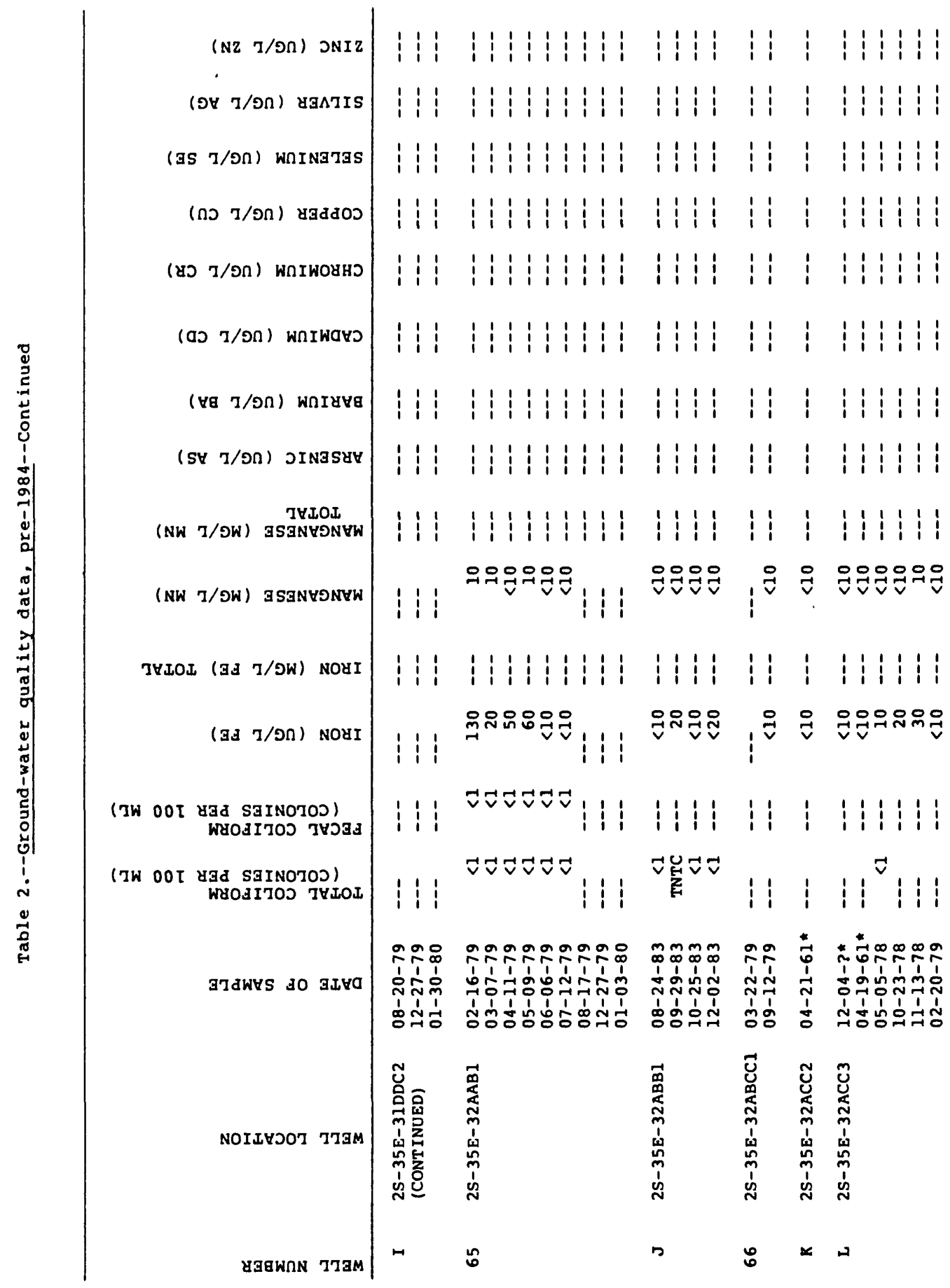




\begin{tabular}{|c|c|c|c|c|c|}
\hline$(\mathrm{T} / \mathrm{DW}) \mathrm{doJ}$ & 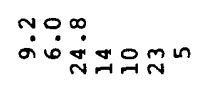 & 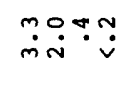 & mi̊n & 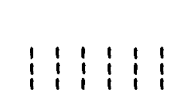 & $1: 11$ \\
\hline 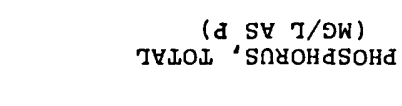 & 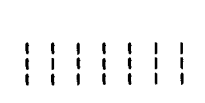 & 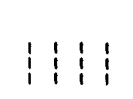 & 151 & 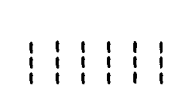 & $\begin{array}{ll:l}1 & 1 & 1 \\
1 & 1 & \vdots\end{array}$ \\
\hline 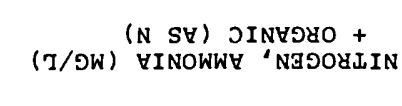 & $\begin{array}{llll:lll}1 & 1 & 1 & 1 & 1 & 1 & 1 \\
1 & 1 & 1 & 1 & 1\end{array}$ & $1: 151$ & $\ddot{0}: \mid$ & 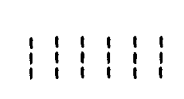 & 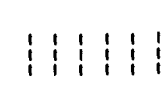 \\
\hline $\begin{array}{c}(N S Y T / S W) \\
\text { GLUZIIN }\end{array}$ & $\begin{array}{lllllll}1 & 1 & 1 & 1 & 1 & 1 & 1 \\
1 & 1 & 1 & 1 & 1\end{array}$ & $1 !$ & $\begin{array}{l:l}1 & 1 \\
1 & 1\end{array}$ & $\begin{array}{llllll}0 & \\
0 & 1 & 1 & 1 & 1 & 1\end{array}$ & 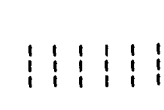 \\
\hline $\begin{array}{c}\text { (N SUT/OW) } \\
\text { HINOWW “NGSO\&IIN }\end{array}$ & $\begin{array}{llll:lll}1 & 1 & 1 & 1 & 1 & 1 & \vdots \\
1 & 1 & 1 & 1 & 1\end{array}$ & $\vdots$ & 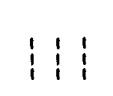 & 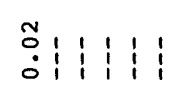 & 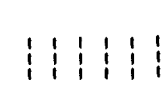 \\
\hline (ZOIS S甘 I/JW) 甘JITIS & $\begin{array}{llll:l:l}1 & 1 & 1 & 1 & 1 & 1\end{array}$ & $1: 1 \vdots$ & $1: 1$ & 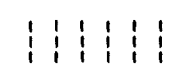 & 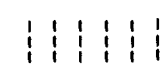 \\
\hline (bos SH T/פW) GLUJTnS & NN|N⿴囗十 & $1: 15:$ & $1: 1$ & 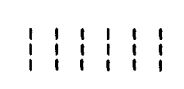 & 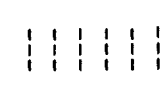 \\
\hline (т $\mathrm{SH} T / \mathrm{TW}$ ) эагषотно & an: & $1 \vdots 1 \vdots$ & $1 \vdots \vdots$ & 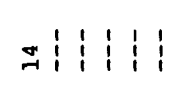 & 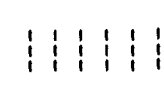 \\
\hline (a SU I/OW) gargonTa & 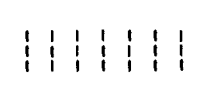 & $\begin{array}{l:ll}1 & 1 & 1 \\
1 & 1 & 1\end{array}$ & $1 \vdots \vdots$ & $\stackrel{5}{5}: 1: 11:$ & $\begin{array}{lll:l}1 & 1 & 1 & 1 \\
1 & 1\end{array}$ \\
\hline ( & 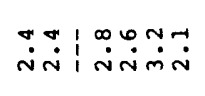 & $\dot{m} \dot{m} \dot{m} \dot{\sim} \dot{m}$ & $\stackrel{\infty}{\dot{m} \dot{m} \dot{n}}$ & 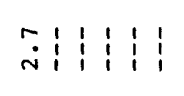 & $\begin{array}{llll}1 & 1 & 1 & \vdots \\
1 & 1 & 1\end{array}$ \\
\hline ( $\mathrm{YN} S \mathrm{SH} T / \mathrm{DW}$ ) wnICOS & $\exists=1000 \infty$ & 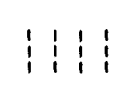 & $1: \vdots \vdots$ & 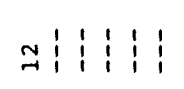 & 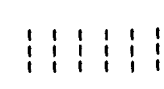 \\
\hline (פW SH T/פW) WกISGNפYW & デ:゚ュュュ & 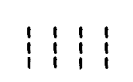 & $1: 1$ & 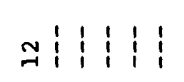 & $\begin{array}{lllll}1 & 1 & 1 & 1 & 1 \\
1 & 1 & 1 & 1\end{array}$ \\
\hline 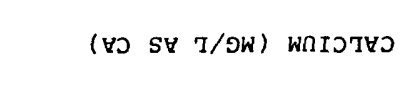 & 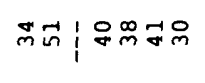 & $1: 1:$ & $1: 11$ & in: $11: 11$ & 14111 \\
\hline 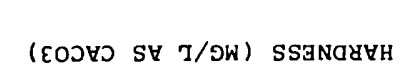 & 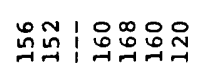 & 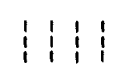 & $\begin{array}{l}1 \\
1 \\
1\end{array}$ & 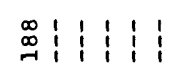 & $\begin{array}{llllll}1 & 1 & 1 & 1 \\
1 & 1 & 1 & 1\end{array}$ \\
\hline 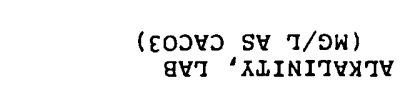 & 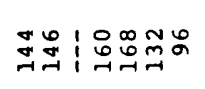 & 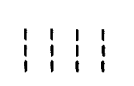 & $1: 1$ & 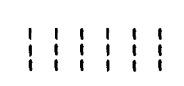 & $\begin{array}{llllll}1 & 1 & 1 & 1 & 1 \\
1 & 1 & 1 & 1 & 1\end{array}$ \\
\hline (SLIN GYYGNULS) Hd & 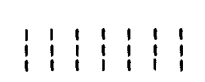 & 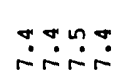 & 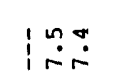 & 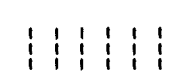 & $\begin{array}{ll:l}1 & 1 & 1\end{array} \mid$ \\
\hline D. 08t LY anaisay 'saitos & 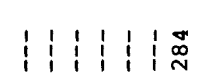 & 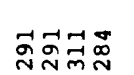 & $1 \stackrel{\infty}{\stackrel{\infty}{N}}$ & 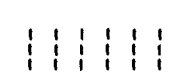 & 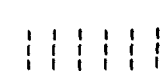 \\
\hline 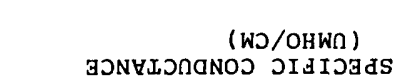 & $\begin{array}{llllll:l}1 & 1 & 1 & 1 & 1 & 1 & \vdots\end{array}$ & 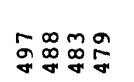 & $\mid$\begin{tabular}{l}
$\sigma \infty$ \\
\multirow{\sigma}{*}{$\%$}
\end{tabular} & 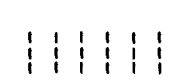 & $\begin{array}{ll:ll}1 & 1 & 1 & 1 \\
1 & 1 & 1 & 1\end{array}$ \\
\hline ХวNตอบ อNIนว & $x x x x x x$ & 以国 & $\omega \omega$ & 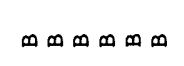 & $x=$ \\
\hline (LGGA ) HLdGa ТTヨM TULOL & : & $i$ & 1 & $i$ & $\stackrel{n}{6}$ \\
\hline 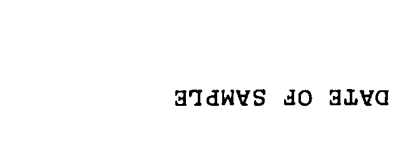 & 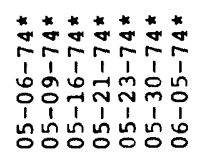 & 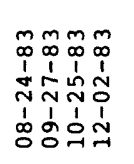 & 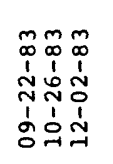 & 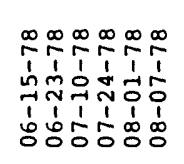 & 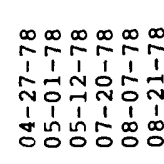 \\
\hline NOILพวOT TTaM & 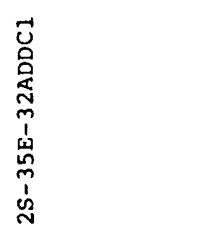 & 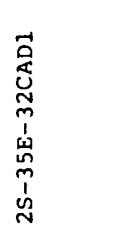 & 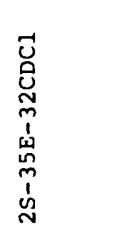 & 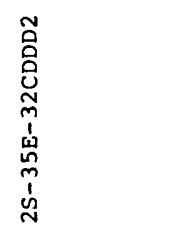 & 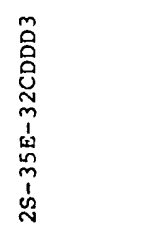 \\
\hline \&ฐgWกN тЈaм & $\approx$ & $\stackrel{\circ}{\sim}$ & - & $z$ & $\circ$ \\
\hline
\end{tabular}




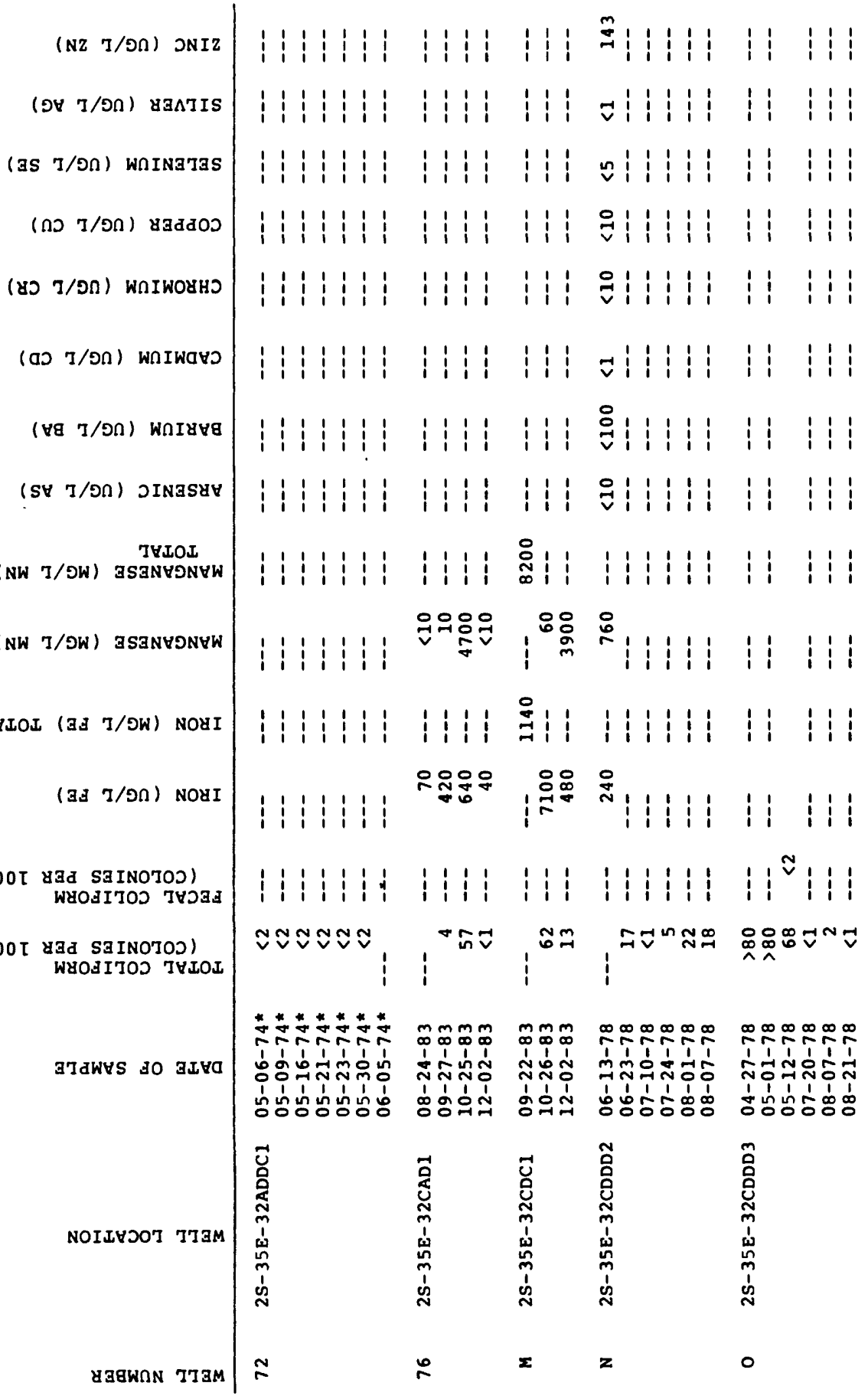




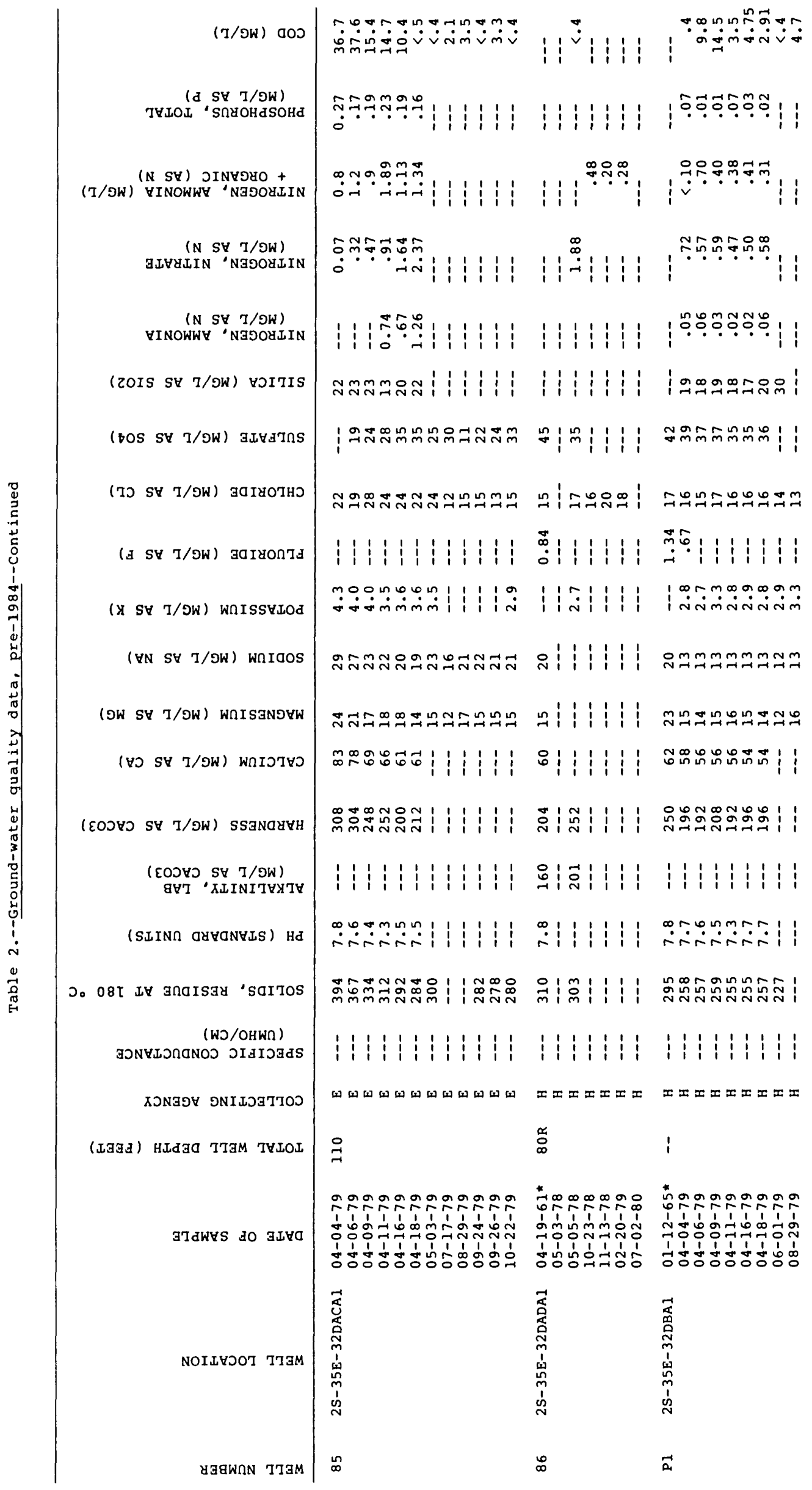




\begin{tabular}{|c|c|c|}
\hline ( NZ I/ON) JNIZ & 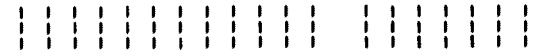 & $\begin{array}{l}i \\
i\end{array} i 1 i$ \\
\hline 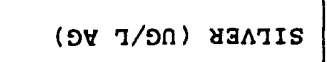 & 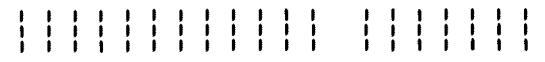 & 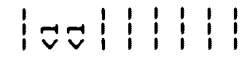 \\
\hline (as $t / 9 n$ ) wกINajas & 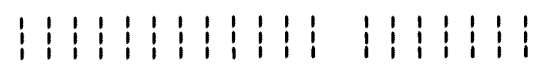 & 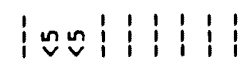 \\
\hline ( $7 / 9 n)$ yaddos & 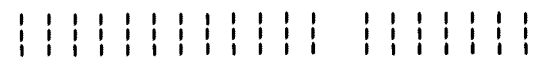 & 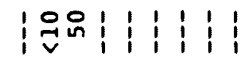 \\
\hline 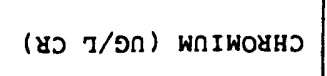 & 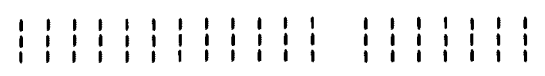 & !号守：１：： \\
\hline (a) $T / 9 n$ ) wnIwaro & 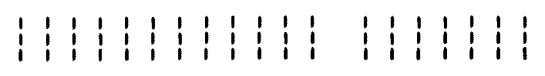 & 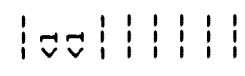 \\
\hline 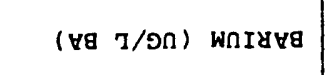 & 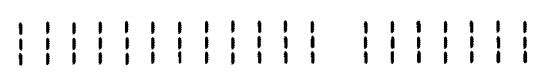 & 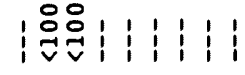 \\
\hline (SH T/OR) OINGSUY & 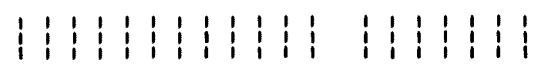 & 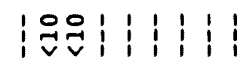 \\
\hline (NW T/OW) $\begin{array}{c}\text { THLOL } \\
\text { JSANYSWW }\end{array}$ & 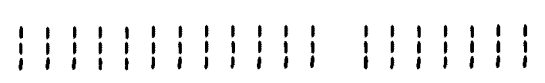 & $1: 1:$ \\
\hline (NW T/OW) JS3NYONYW & 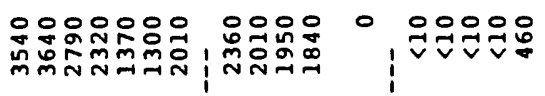 & 룸욱운도 \\
\hline IYIOL (AS $7 /$ OW) NOHI & 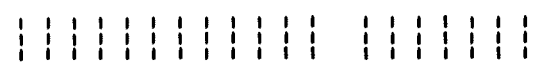 & $\begin{array}{llll}1 & 1 & 1 & 1 \\
1 & 1 & 1 & 0\end{array}$ \\
\hline$(33 T / 90)$ NOYI & 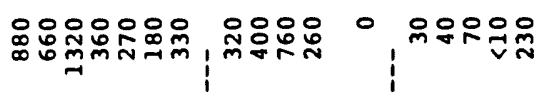 & 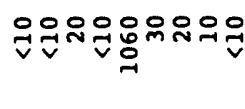 \\
\hline $\begin{array}{l}\text { OOI Yad SBINOTOJ) } \\
\text { WXOAITOJ THJas }\end{array}$ & 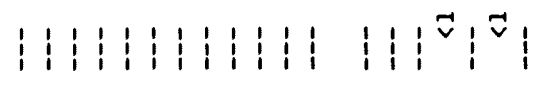 & 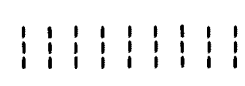 \\
\hline $\begin{array}{l}\text { OOI YaA SaINOTOJ) } \\
\text { WYOSIJOS TYLOI }\end{array}$ & 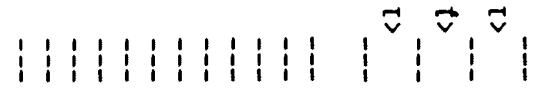 & $1: 1$ \\
\hline 37dWYS so aIta & 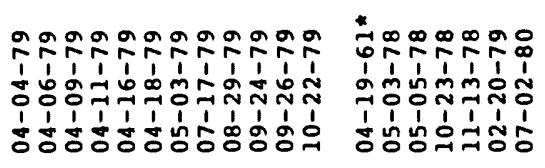 & 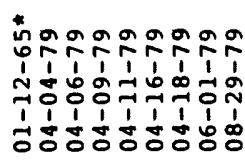 \\
\hline NOIIYJOT TIJM & 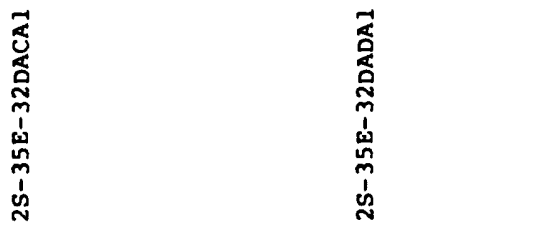 & 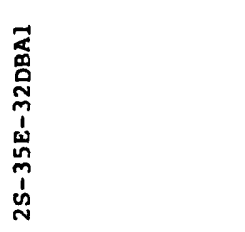 \\
\hline צง8WnN Tว & 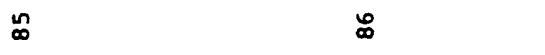 & a \\
\hline
\end{tabular}




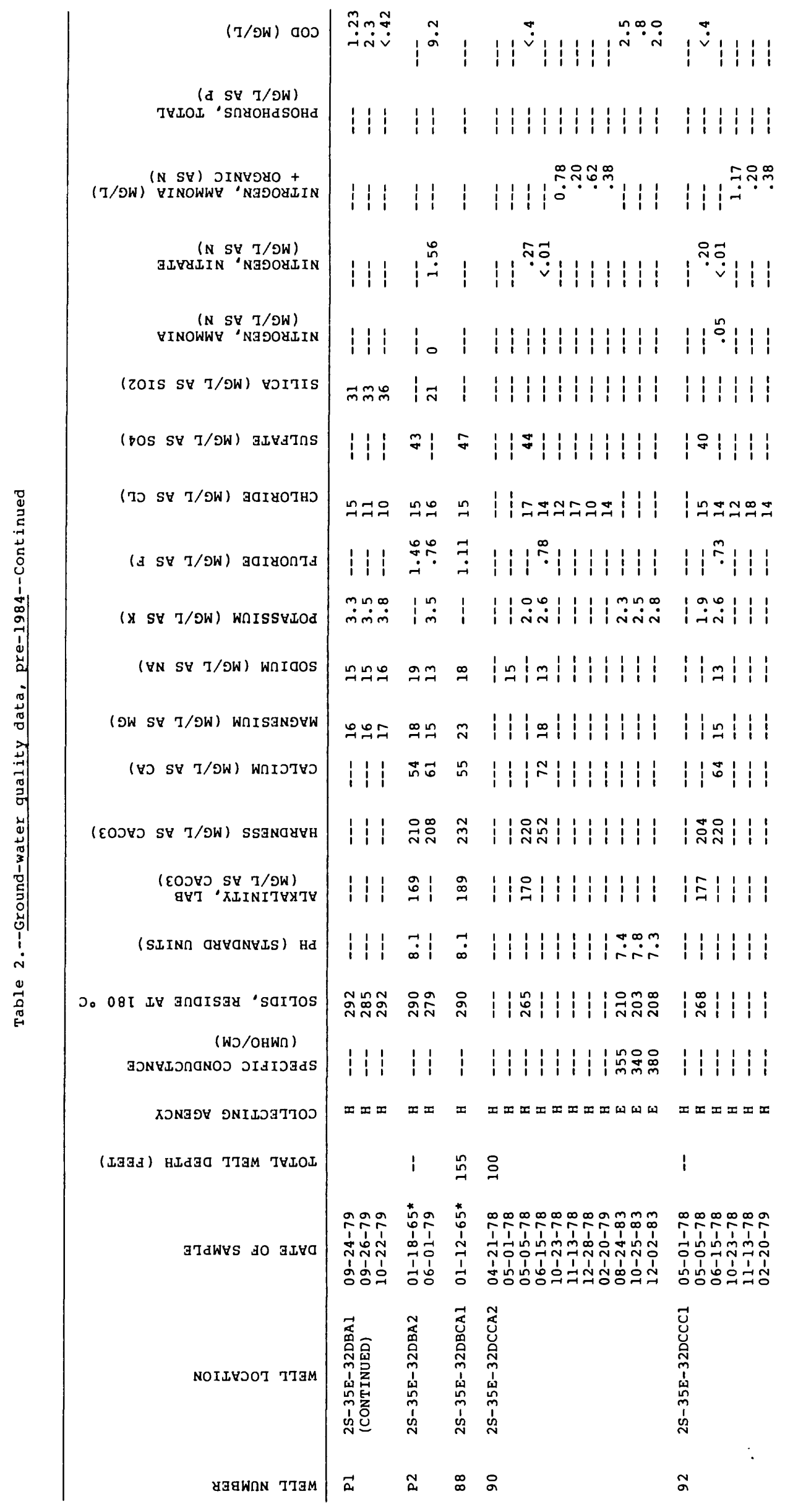




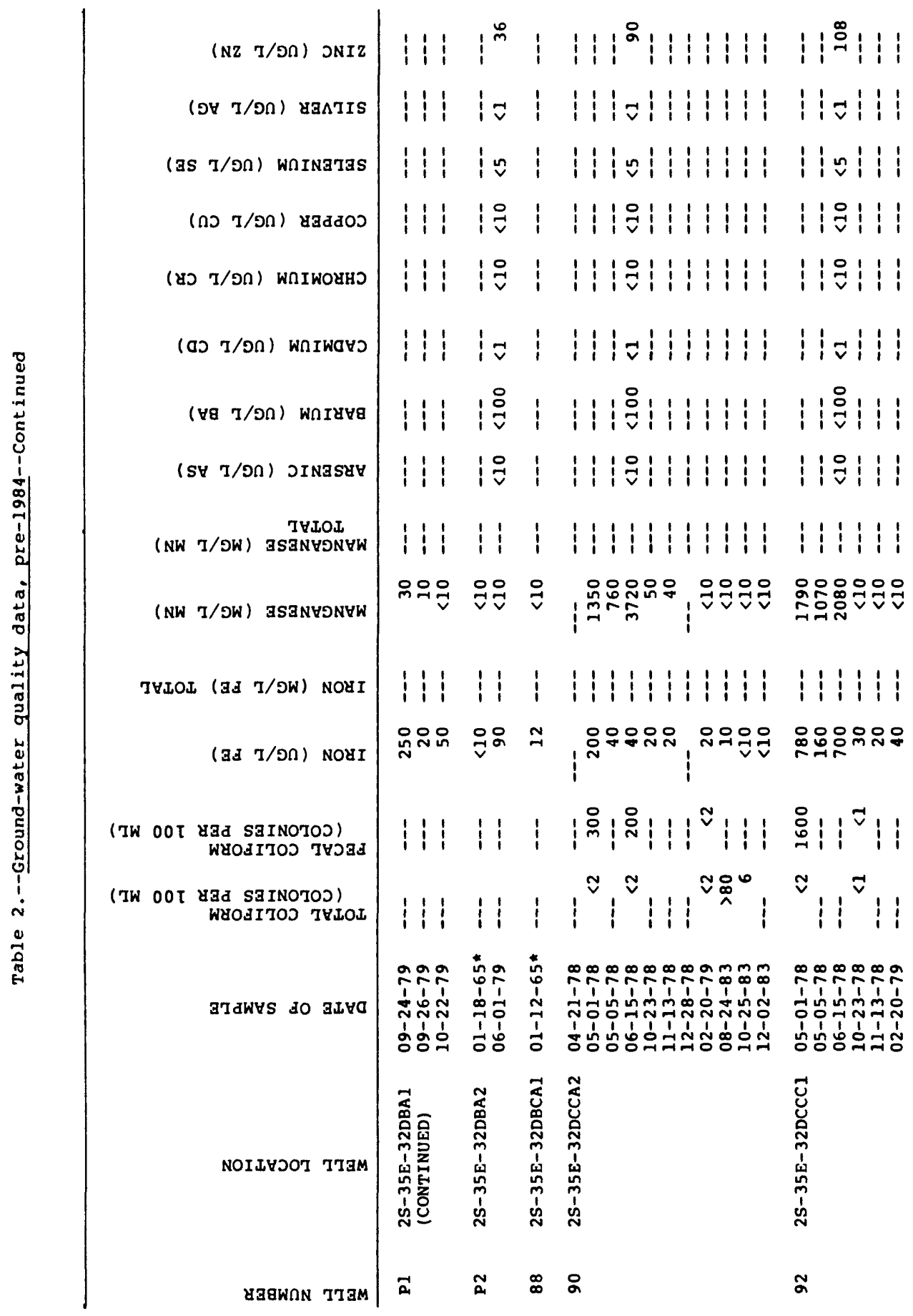




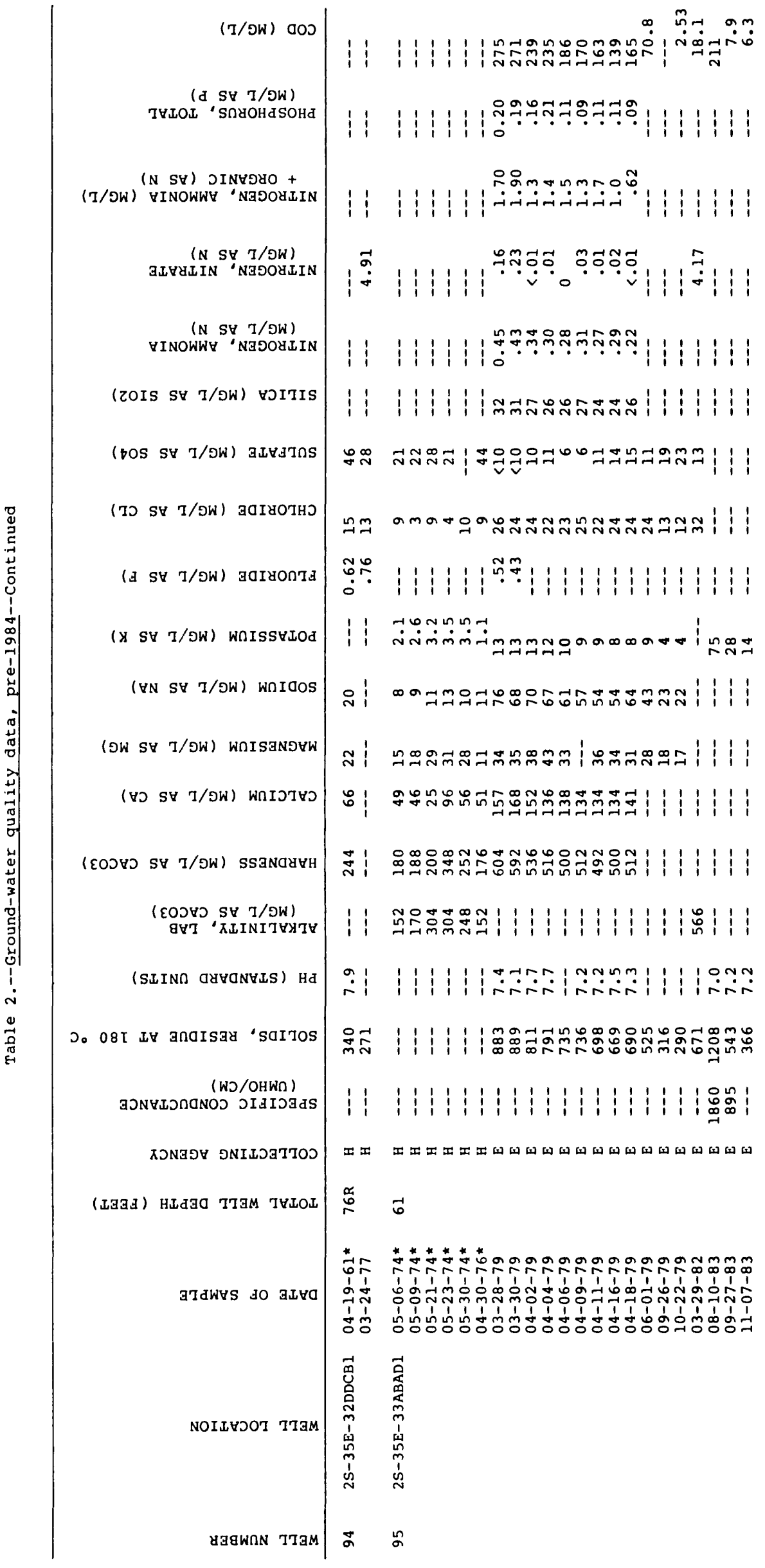




\begin{tabular}{|c|c|}
\hline (NZ T/OR) JNIZ & 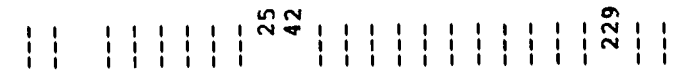 \\
\hline 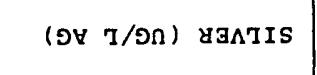 & 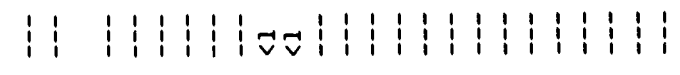 \\
\hline (as $T / O R$ ) WRINGTas & 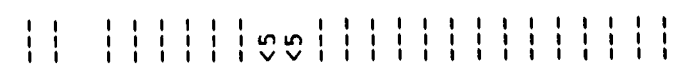 \\
\hline 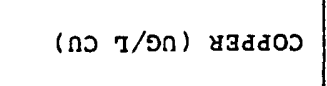 & 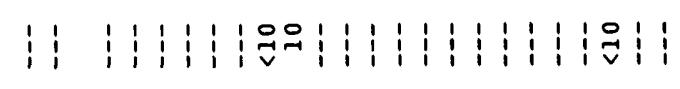 \\
\hline (ะว $3 / 9 \cap$ ) พกIพоชHอ & 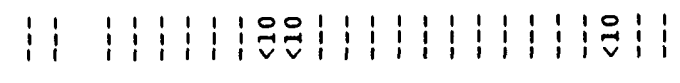 \\
\hline (םכ T/פn) wกIwaษ & 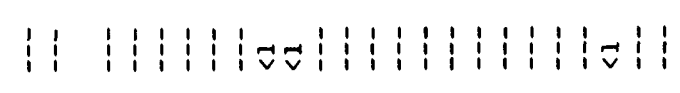 \\
\hline (ษg $3 / 9 n$ ) wกIษษ & 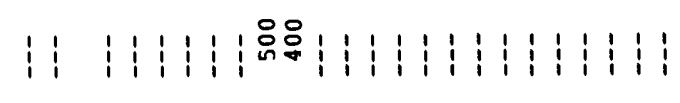 \\
\hline (SY T/OR) JINGSUY & ! \\
\hline $\begin{array}{c}\text { TYIOI } \\
(\text { NW } I / O W) \text { ISGNYNYW }\end{array}$ & 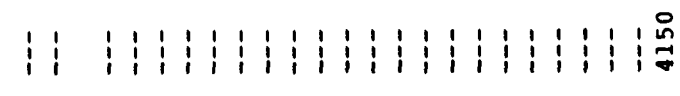 \\
\hline (NW $I / O W)$ aSaNYONYW & ㄴ \\
\hline IYIOJ (3a $\mathrm{I} / \mathrm{OW}$ ) NOYI & 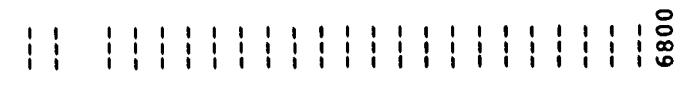 \\
\hline (GI $\mathrm{T} / \mathrm{O})$ NOHI & 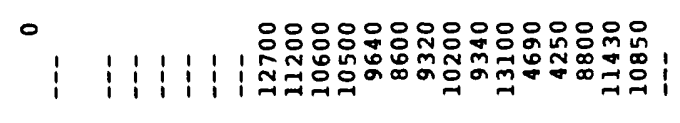 \\
\hline 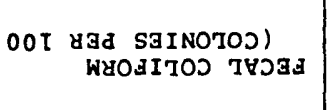 & 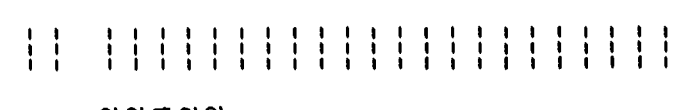 \\
\hline $\begin{array}{l}\text { OOI YGA SBINOTOJ) } \\
\text { WYOAITOJ TYIOI }\end{array}$ & $i j$ \\
\hline gT dWYS so asyd & 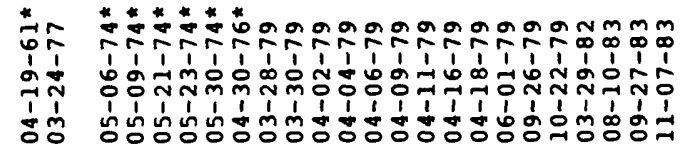 \\
\hline NOILYJO'T TIJM & 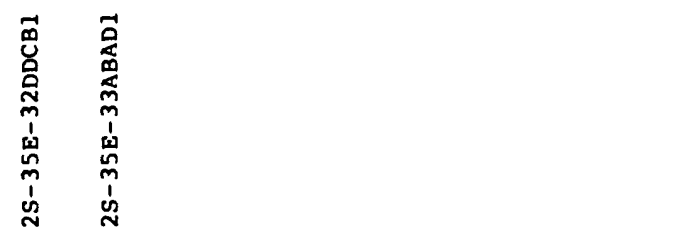 \\
\hline 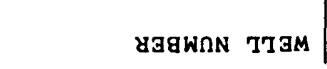 & \\
\hline
\end{tabular}




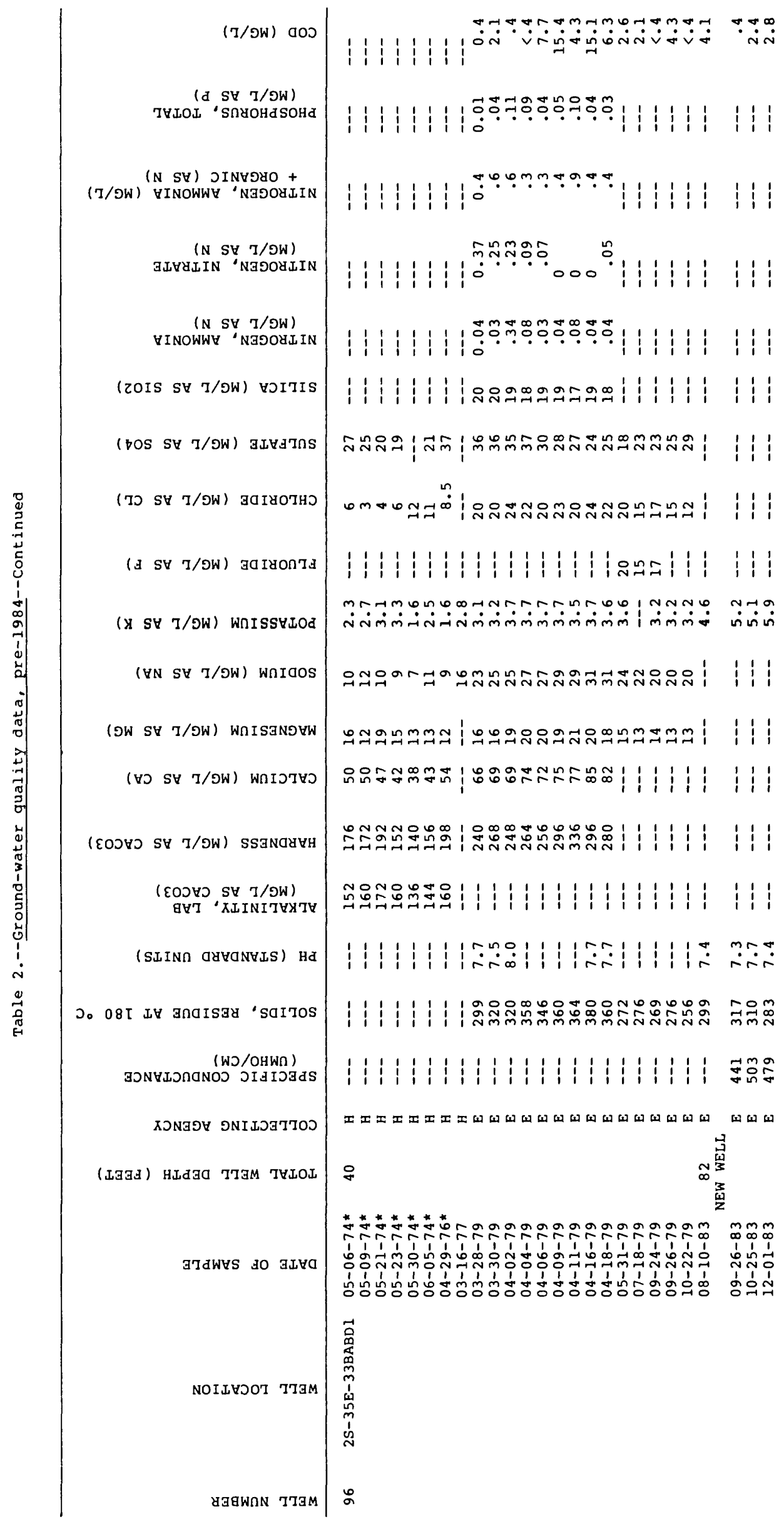




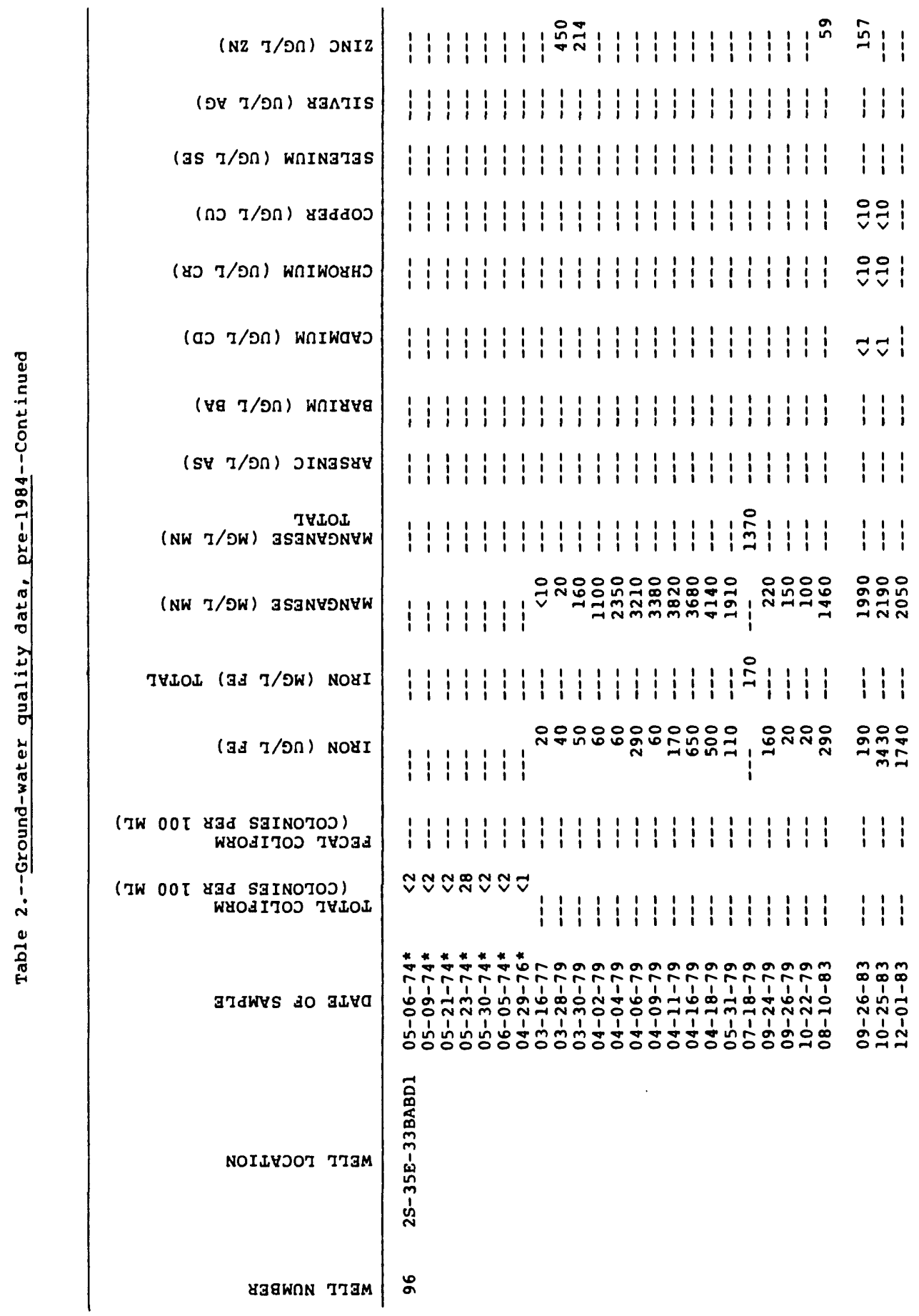




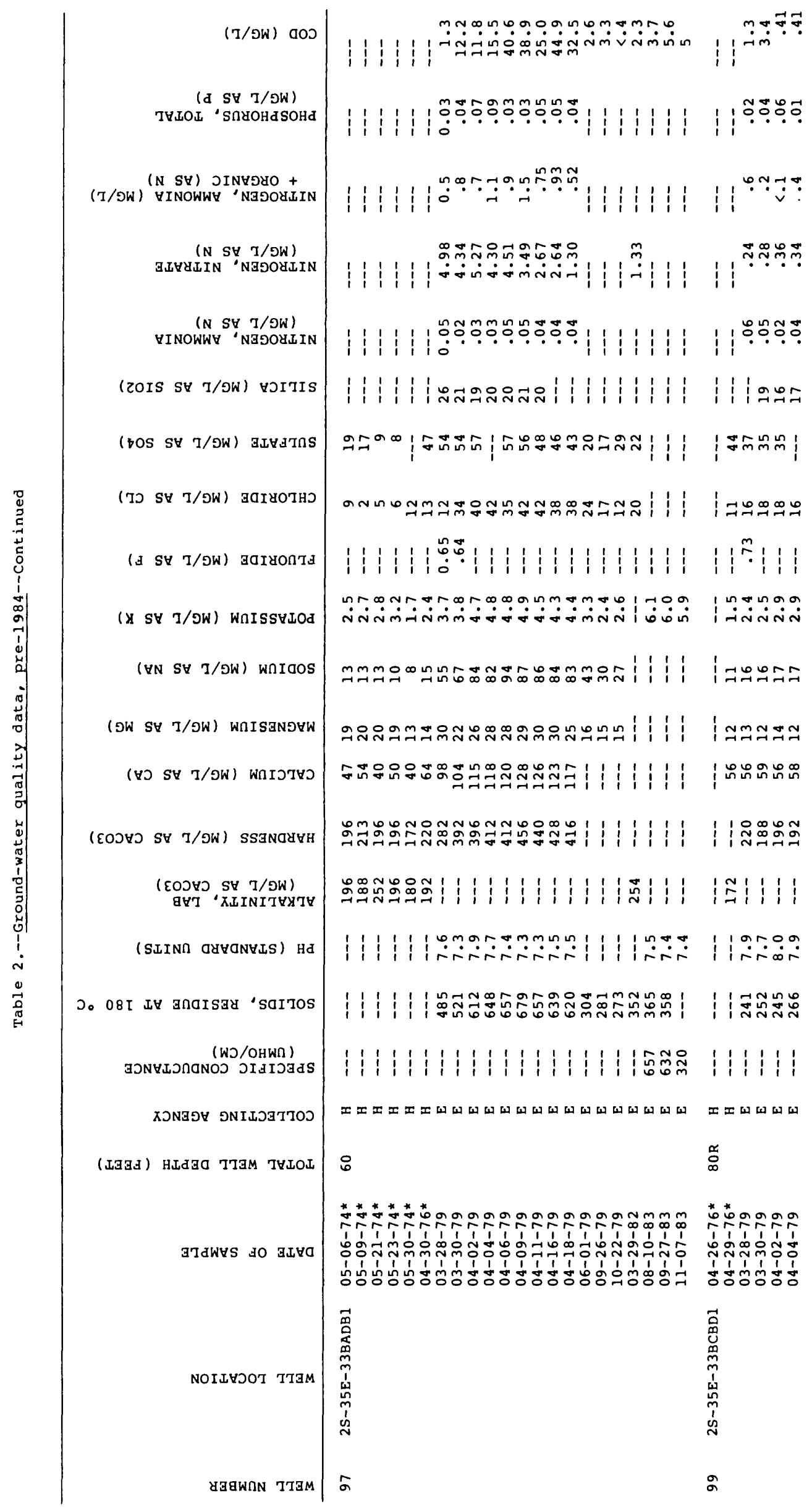




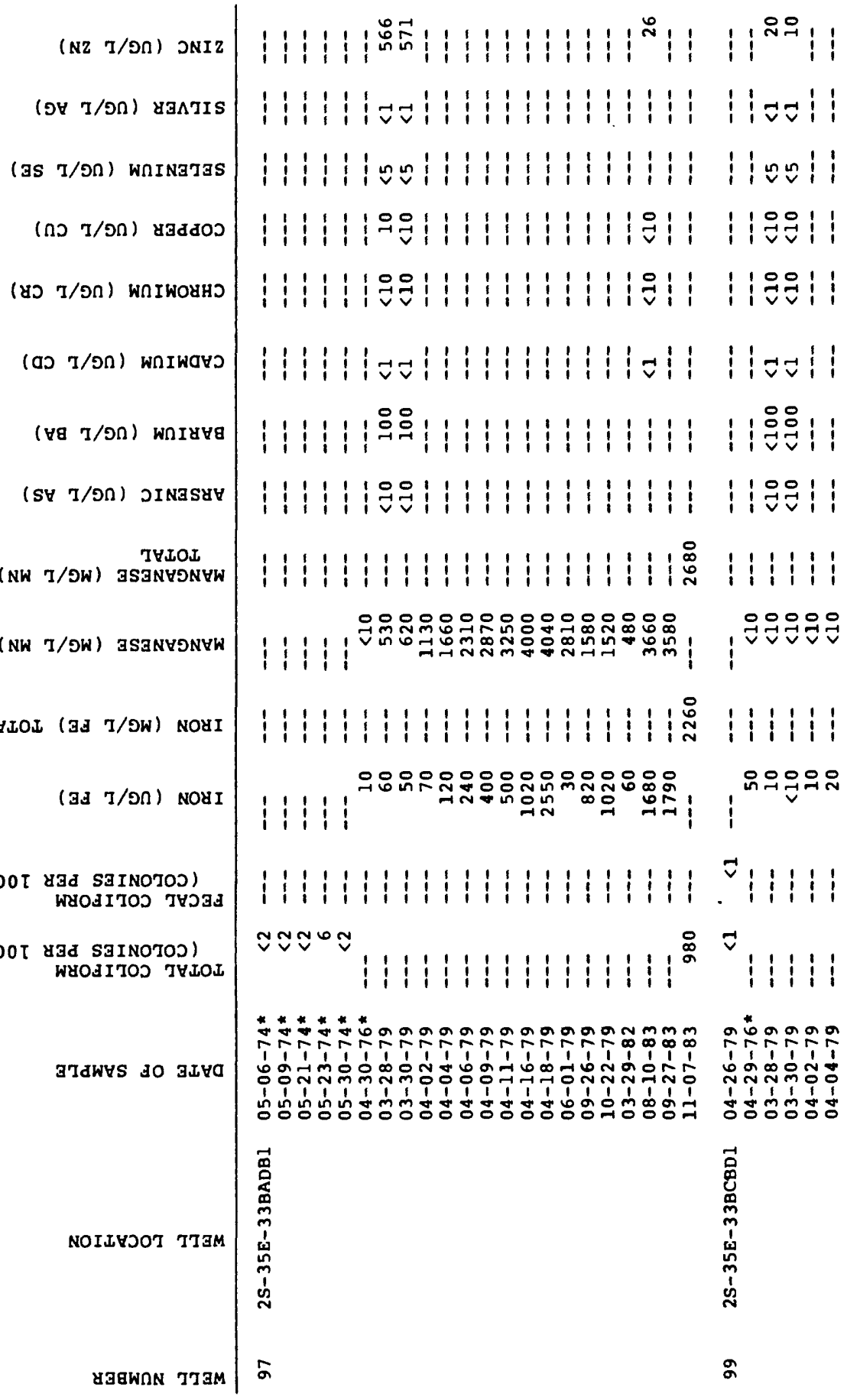




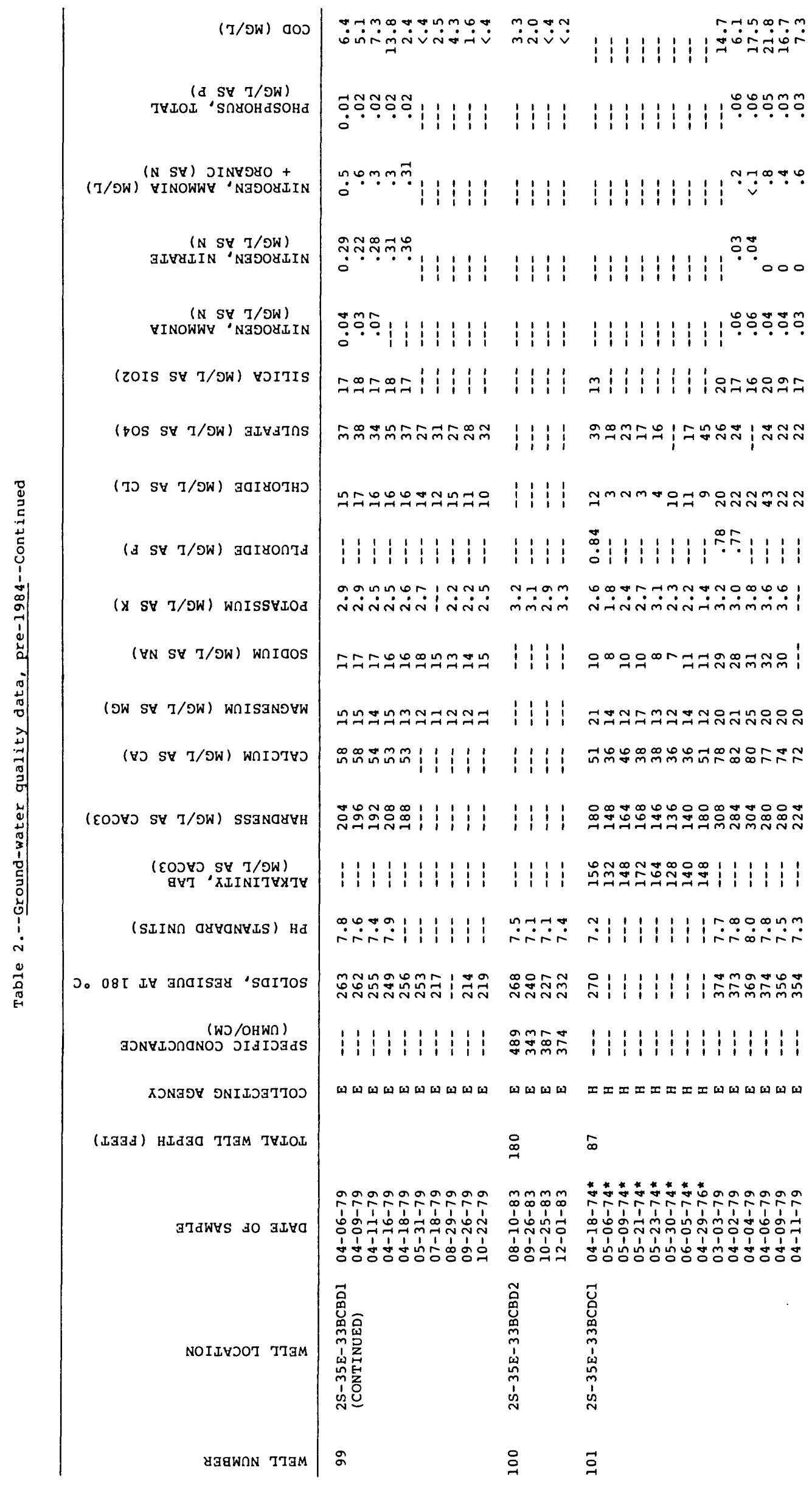




\begin{tabular}{|c|c|c|c|}
\hline (NZ I/OR) JNIZ & $11: 11: 1: 11$ & $=\stackrel{\infty}{ \pm}:$ & 号:11 1 \\
\hline 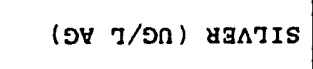 & 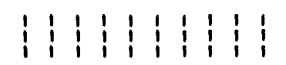 & $\begin{array}{l:ll}1 & 1 & 1 \\
1 & 1\end{array}$ & 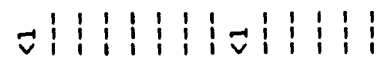 \\
\hline (as $\mathrm{I} / \mathrm{O}$ ) wnINagas & 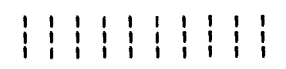 & $\begin{array}{l:l:}1 & 1 \\
1 & 1\end{array}$ & $i i^{n} \vdots$ \\
\hline (חכ $7 /$ כ & 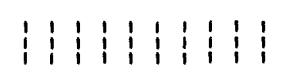 & 踶: & 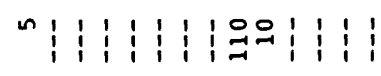 \\
\hline (षכ $J / O n)$ WกIพO\&Hอ & $\begin{array}{llllllllll}1 & 1 & 1 & 1 & 1 & 1 & 1 & 1 & 1 & 1 \\
1 & 1 & 1 & 1 & 1 & 1 & 1 & 1\end{array}$ & $\stackrel{\circ}{*}=1 !$ & 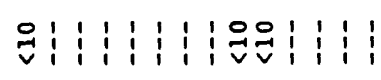 \\
\hline 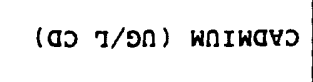 & 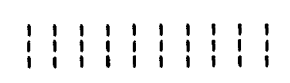 & $\nabla \nabla i !$ & 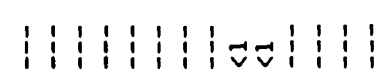 \\
\hline (ษg $7 / O \cap$ ) พกIษษด & 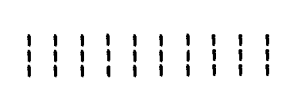 & $\dot{1}$ & 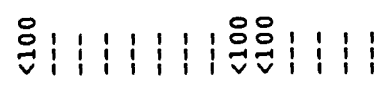 \\
\hline (SH T/OR) JINGSYH & 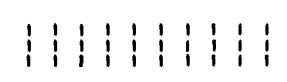 & $1 ! 1 !$ & 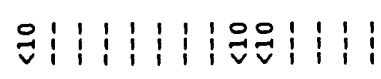 \\
\hline (NW T/OW) $\begin{array}{c}\text { JYIOI } \\
\text { JSanYONYW }\end{array}$ & 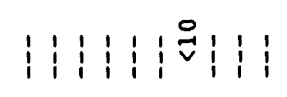 & 1111 & 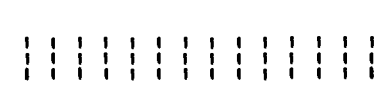 \\
\hline (NW T/OW) ISZNGONYW & 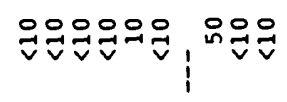 & 00 & $\stackrel{\circ}{\vec{v}}: 1:$ \\
\hline LOI (JA $\mathrm{J} / \mathrm{OW}$ ) NOYI & $1 ! 1: 11^{\circ} \mid 1 !$ & $11: 1$ & 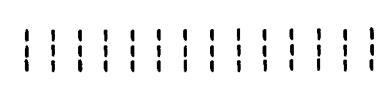 \\
\hline$(3 a T / O n)$ NOdI & 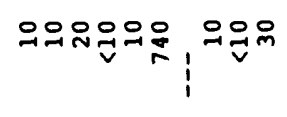 & & 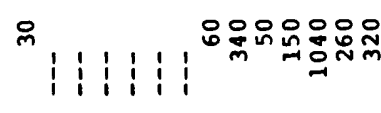 \\
\hline 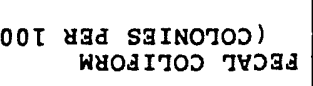 & 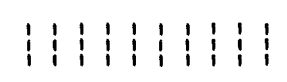 & $1: 11:$ & $1: 1119$ \\
\hline $\begin{array}{l}\text { OOT YGa SaINOTOD) } \\
\text { WYOAIJOS THLOI }\end{array}$ & $1: \vdots$ & $i i^{2}$ & $\mathcal{N}$ \\
\hline JIdWYS do gIVO & 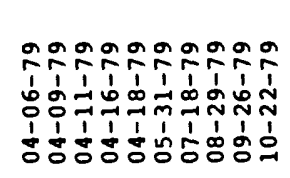 & 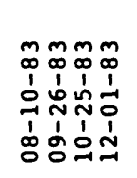 & 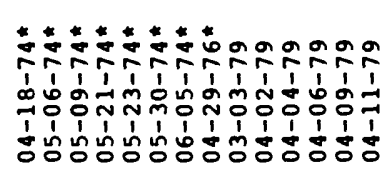 \\
\hline NOILYJOI J7aM & 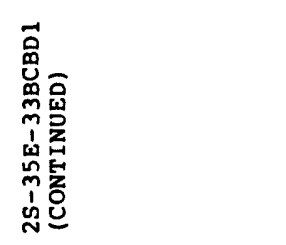 & 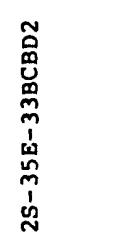 & 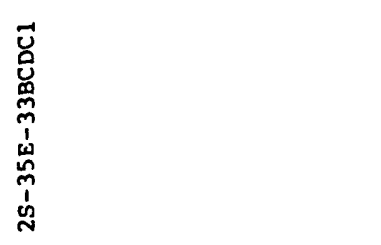 \\
\hline 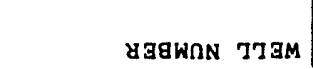 & a & $=$ & \\
\hline
\end{tabular}




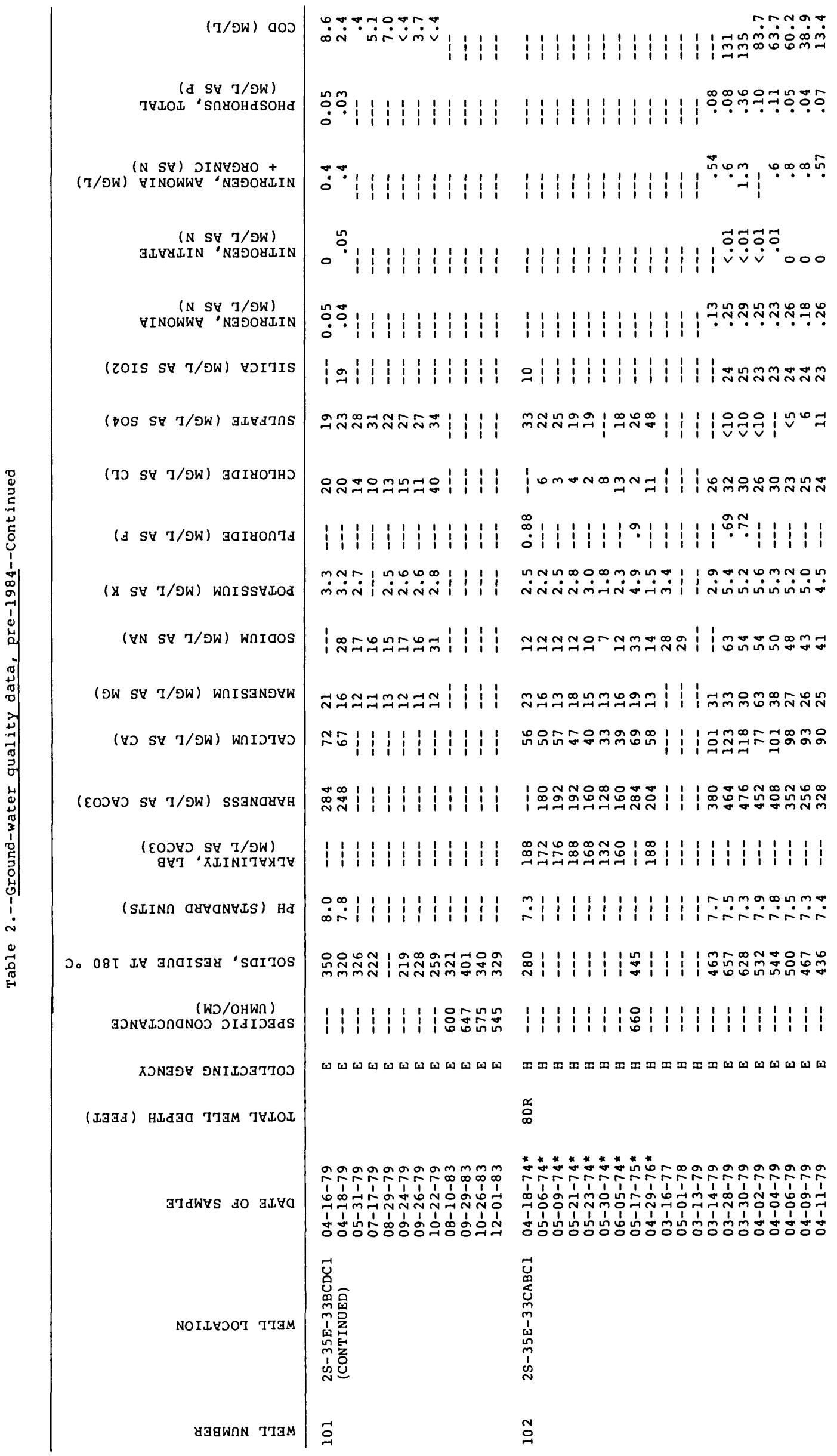




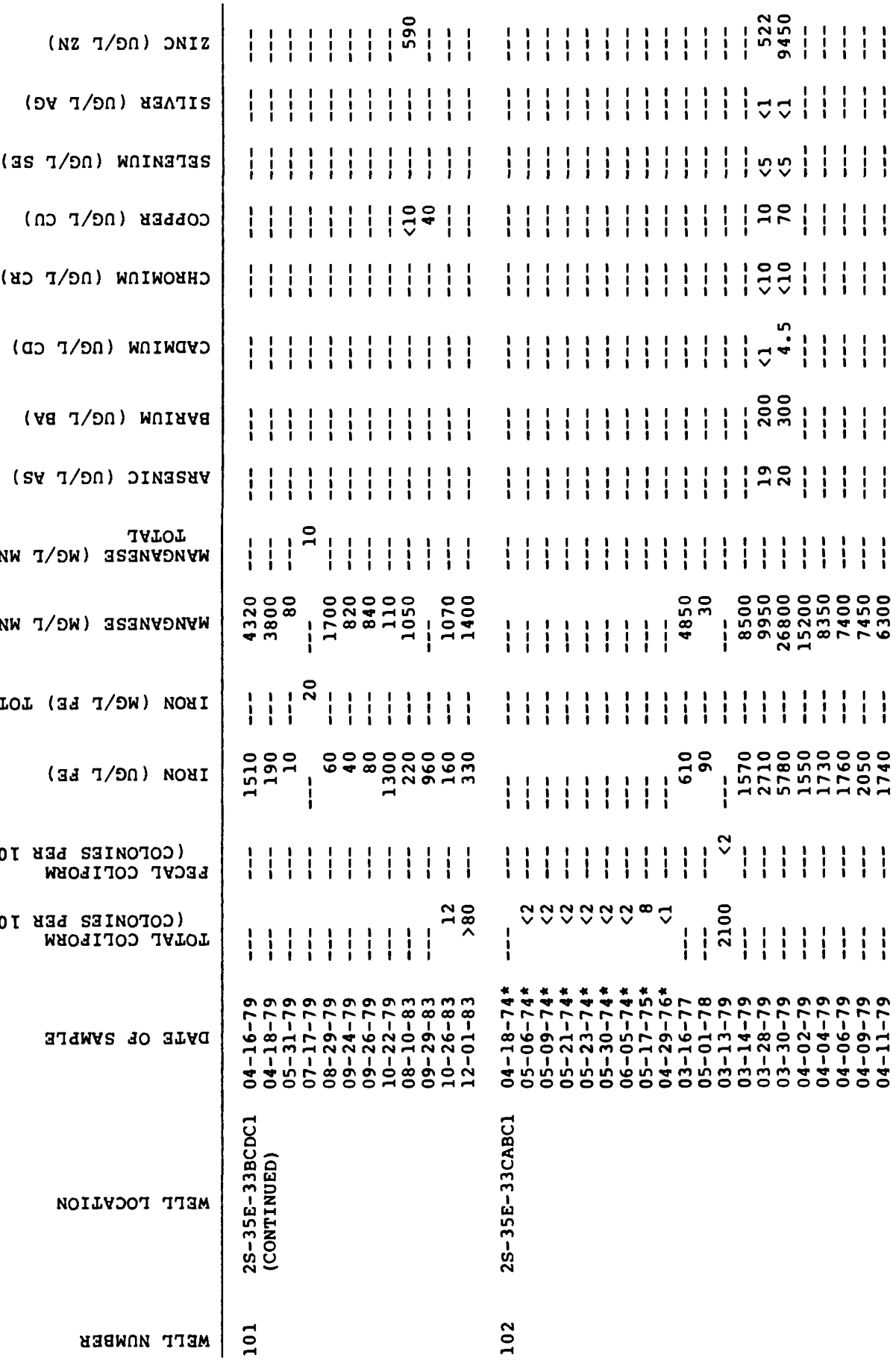




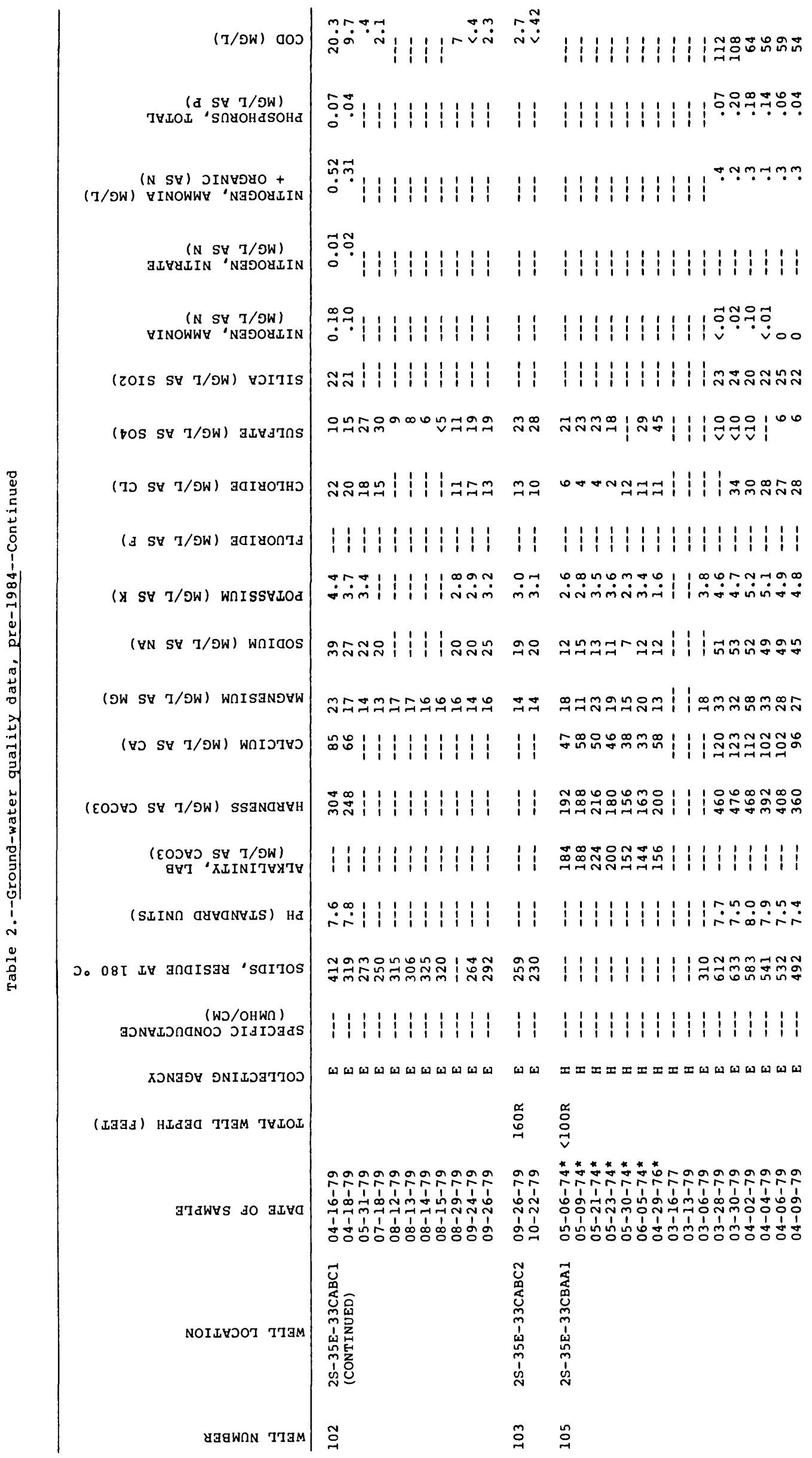




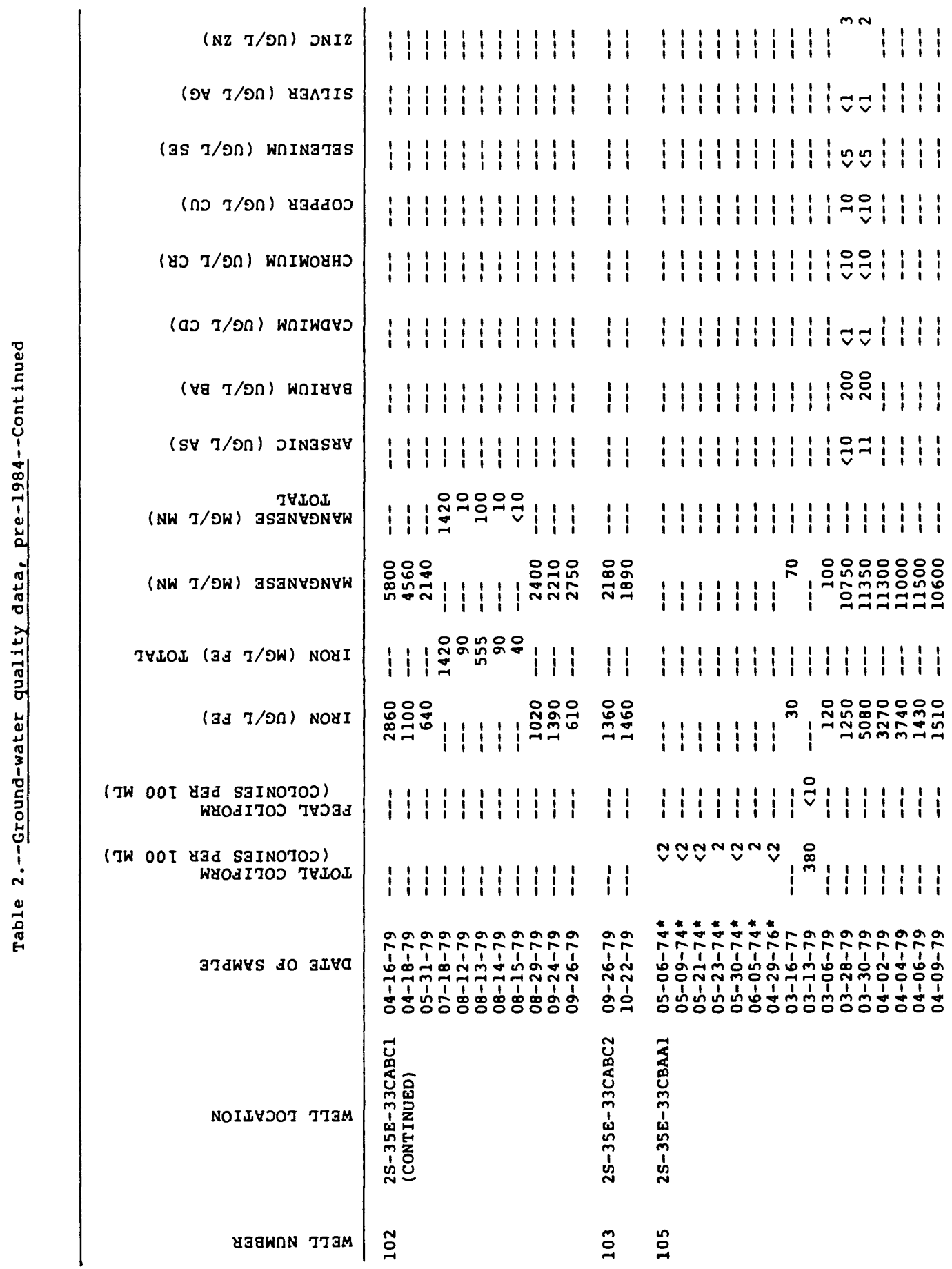




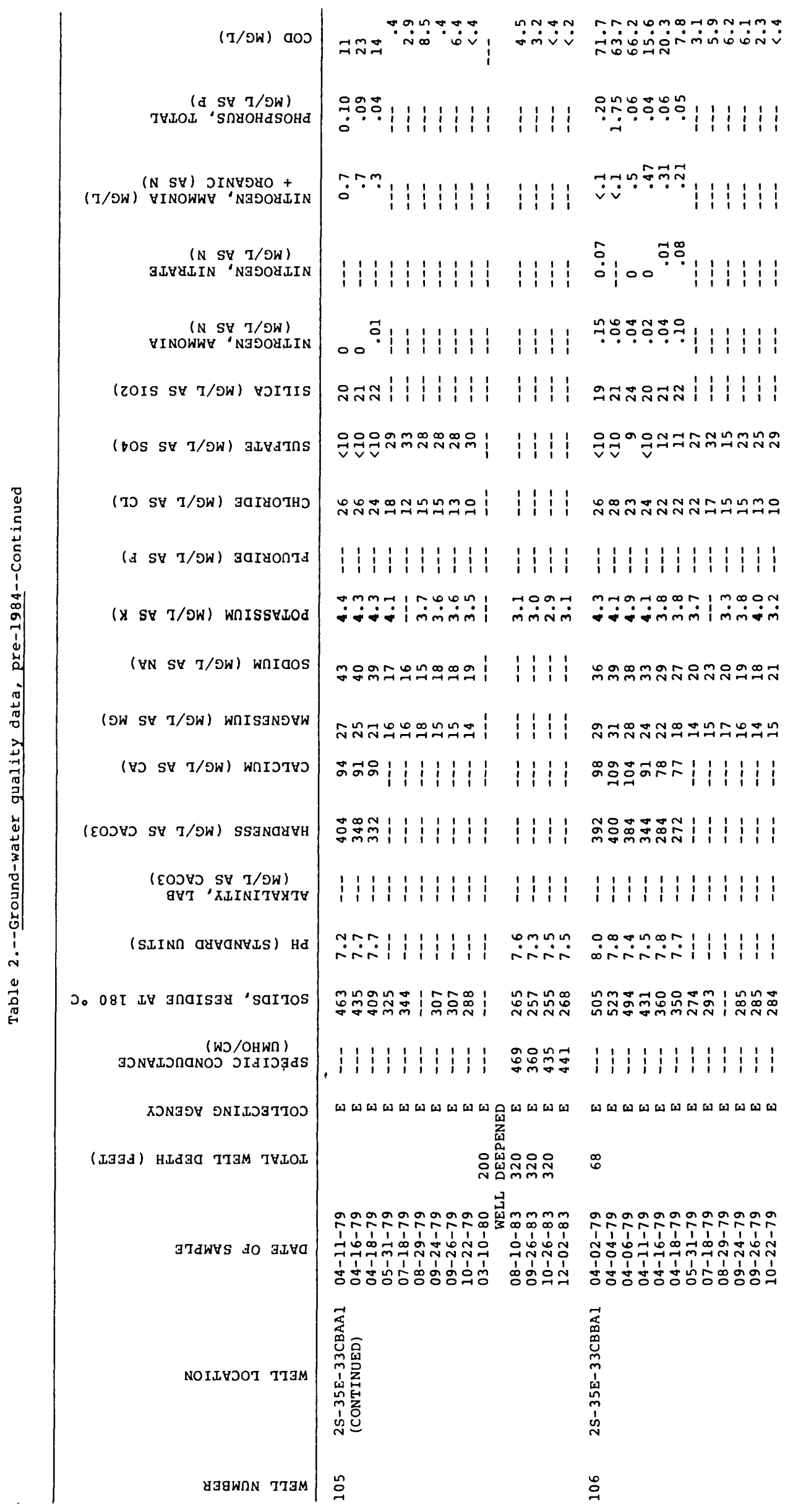




\begin{tabular}{|c|c|c|c|}
\hline (NZ T/ON) JNIZ & 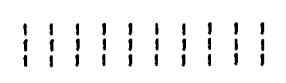 & $\stackrel{*}{*}: \vdots$ & 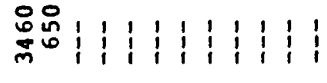 \\
\hline 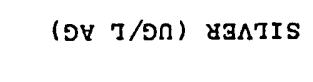 & 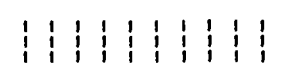 & 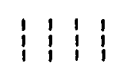 & 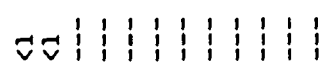 \\
\hline (3s $7 / 0 n$ ) wnINat3s & 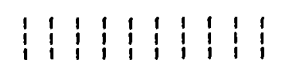 & $1: 1: 1$ & ๓ッ! \\
\hline 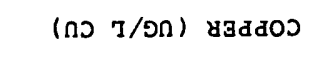 & $\begin{array}{llllllll}1 & 1 & 1 & 1 & 1 & 1 & 1 & 1\end{array}$ & 욱워 & 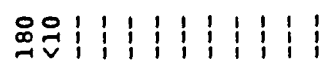 \\
\hline 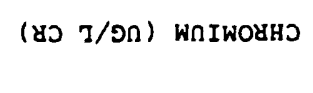 & $\begin{array}{llllllllll}1 & 1 & 1 & 1 & 1 & 1 & 1 & 1 & 1 & 1 \\
\end{array}$ & 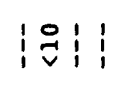 & 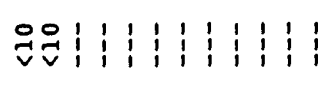 \\
\hline (बכ I/on) wnIwaษ & 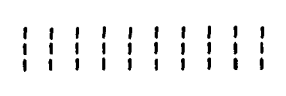 & $i \approx i$ & 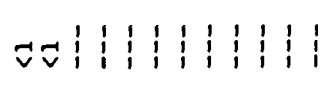 \\
\hline (พg $T / O n)$ พกI4ษ & 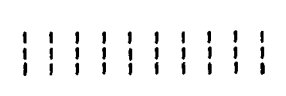 & 1111 & 葛:1 11111111 \\
\hline (SH I/ON) JINGSYH & 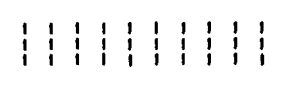 & $1 !$ & 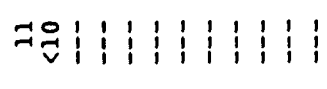 \\
\hline (NW T/OW) $\begin{array}{c}\text { TYIOI } \\
\text { 3SINYONYW }\end{array}$ & $1411^{\circ} 11: 114$ & $1 i$ & 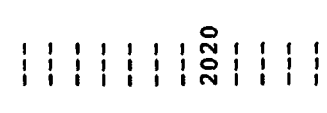 \\
\hline (NW $T / S W$ ) 3SaNYONYW & 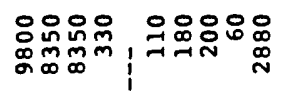 & 웅유 & 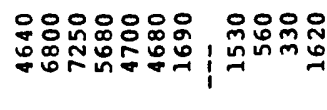 \\
\hline HLOL (as $T / O W$ ) NOYI & 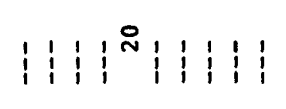 & 1 & 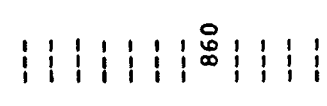 \\
\hline (3a I/On) NOYI & 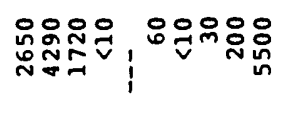 & & 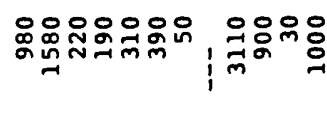 \\
\hline 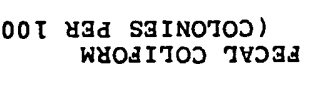 & $\begin{array}{llllllllll}1 & 1 & 1 & 1 & 1 & 1 & 1 & 1 & 1 & 1\end{array}$ & $1: 1: 1$ & $1: 14: 14: 11$ \\
\hline $\begin{array}{l}\text { 00I Y3a S3INOJOJ) } \\
\text { WYOAITOJ TYLOI }\end{array}$ & 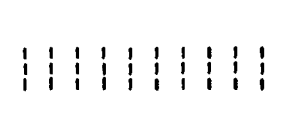 & $1: 1$ & $1 i$ \\
\hline J'dWWS so aIVO & 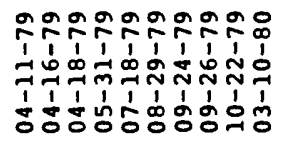 & 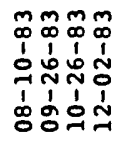 & 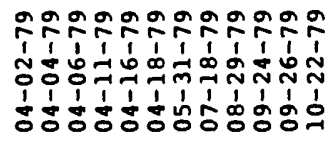 \\
\hline NOIJYDOI $773 M$ & 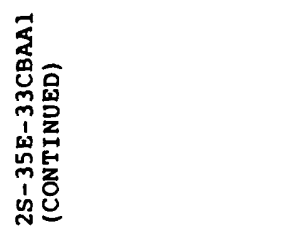 & & 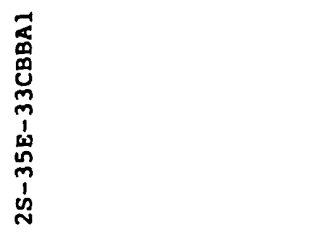 \\
\hline ४งaWnN T'T3M & $\stackrel{2}{\circ}$ & & $\stackrel{0}{\sim}$ \\
\hline
\end{tabular}




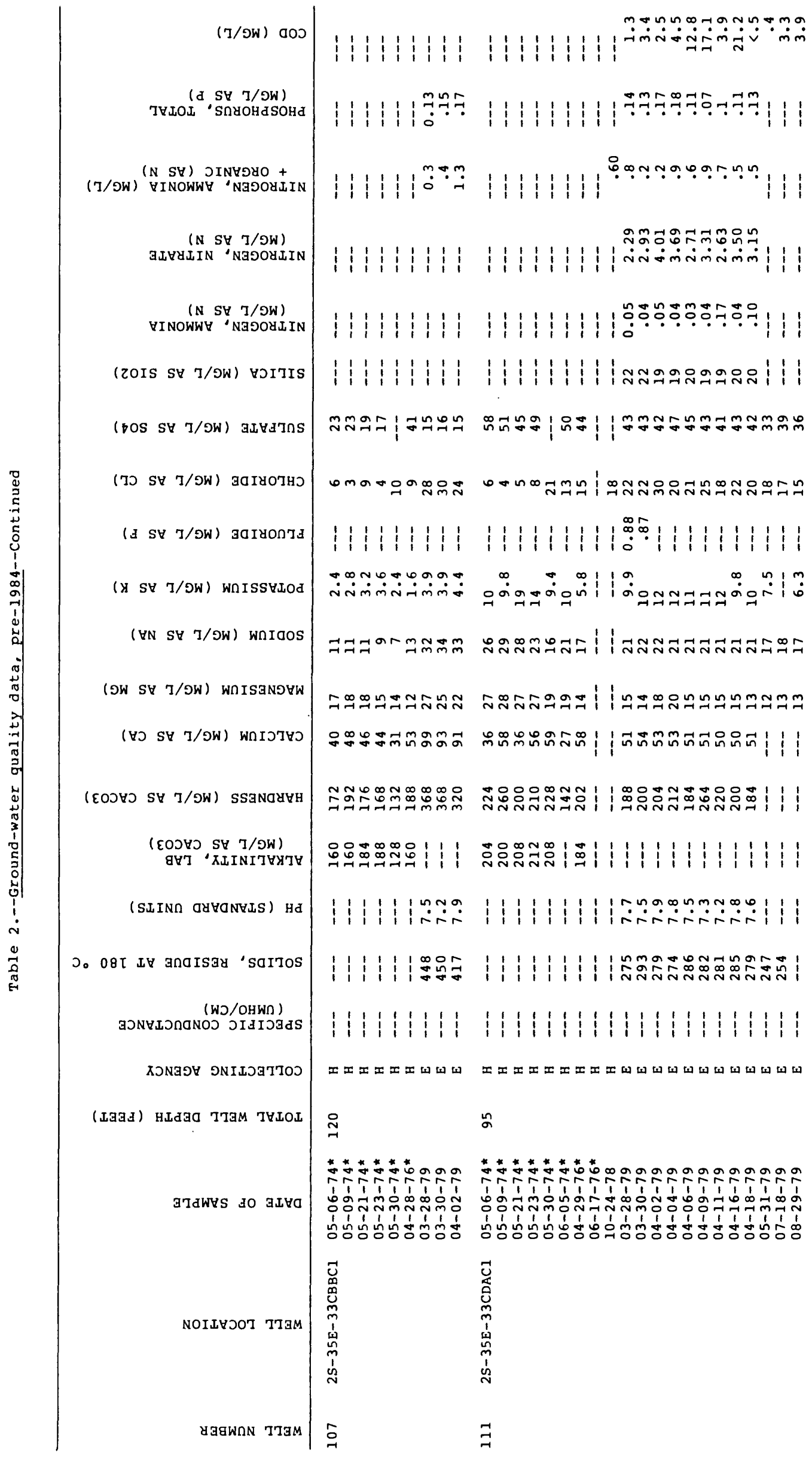




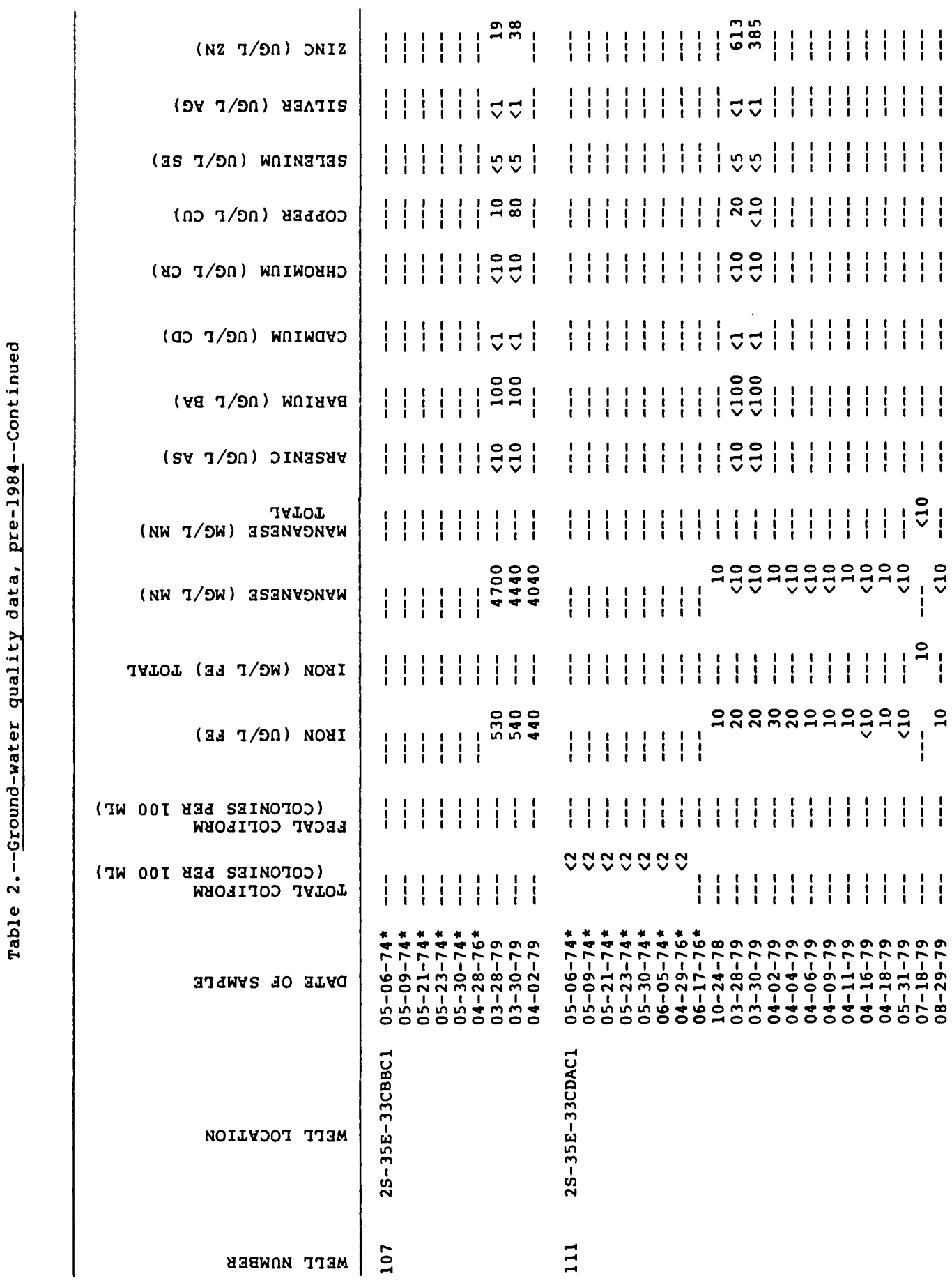




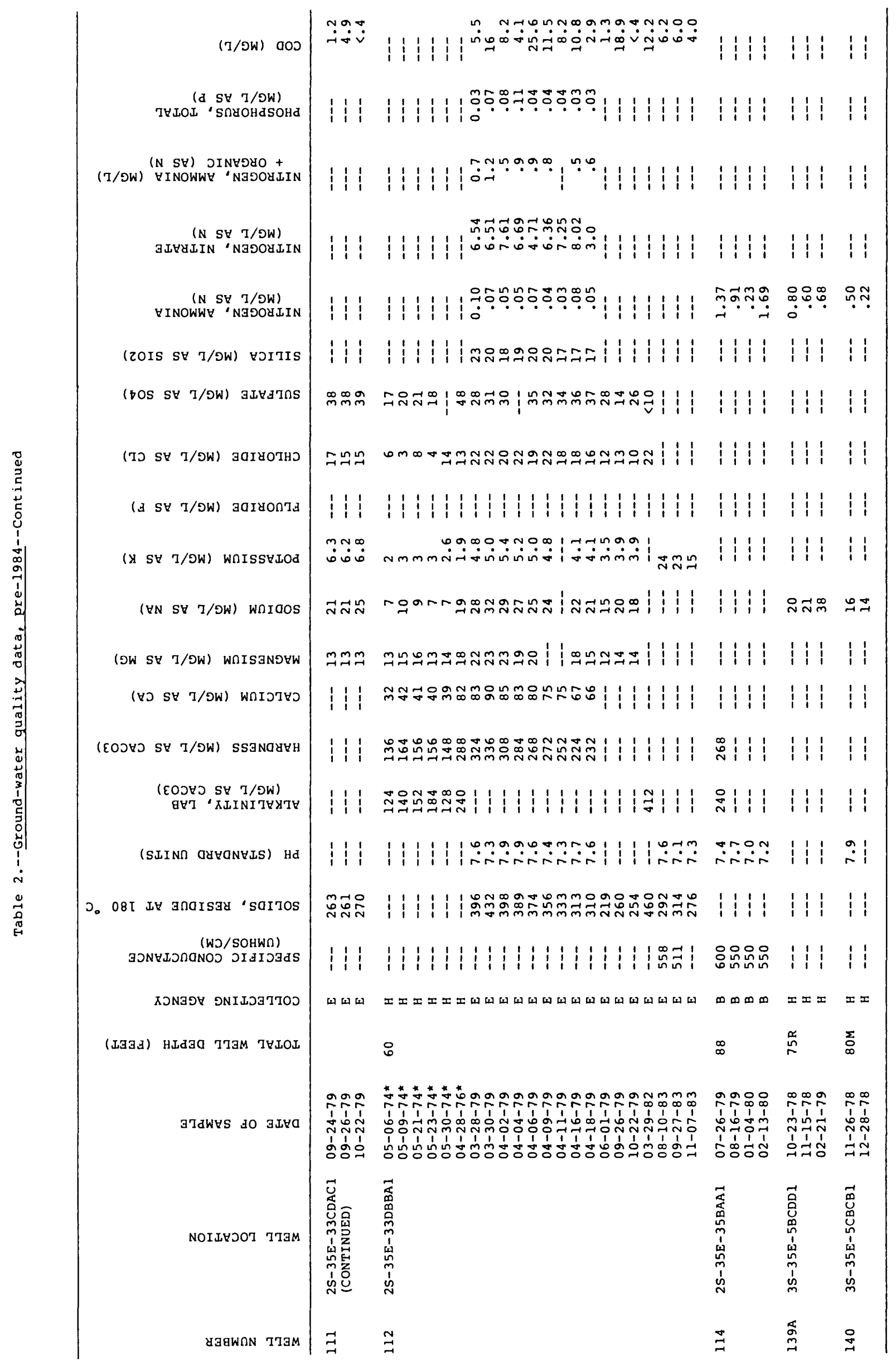




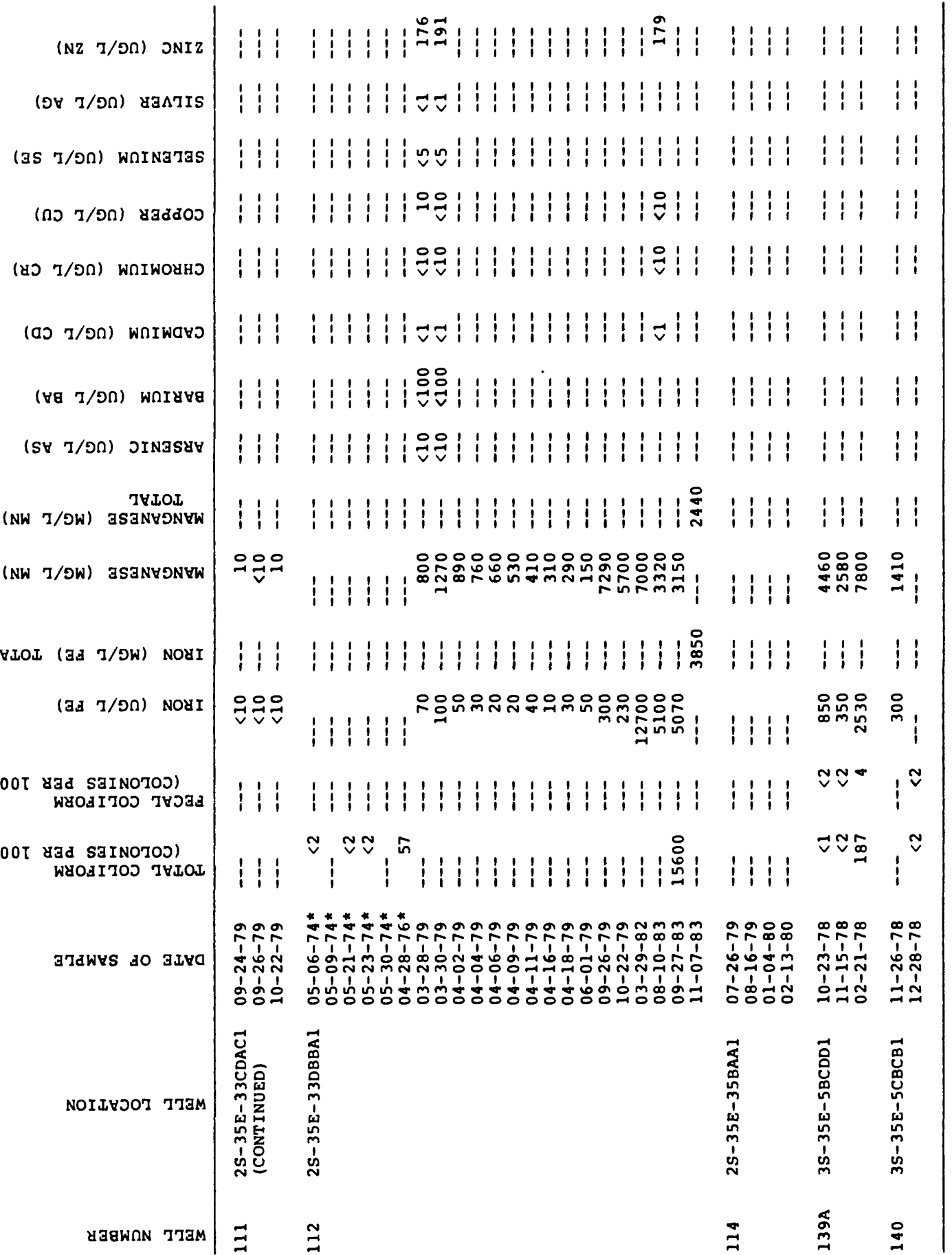


Table 3.--Ground-water quality data, 1984 and 1985

Headnotes

DATE OF SAMPLE: year-month-day

TIME: 24-hour notation

LAB: U.S. Geological Survey Central Laboratory (Denver)

DISSOLVED: Portion of a whole water sample that passes through a 0.45 -micrometer membrane filter

FET-FLD: Fixed endpoint titration, field (onsite) determination

SUSPENDED RECOVERABLE: Amount of constitutent in solution after material retained on a 0.45-micrometer membrane filter has been prepared by a method that results in dissolution of readily soluble substances

TOTAL RECOVERABLE: Amount of constituent in solution after a sample has been prepared by a method that results in dissolution of readily soluble substances

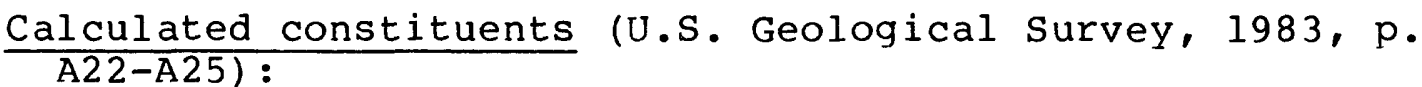

SOLIDS, SUM OF CONSTITUENTS, DISSOLVED: (alkalinity $\mathbf{x} 0.6$ ) + (calcium) + (magnesium) + (sodium) + (chloride) + (sulfate) + (silicate) + (dissolved potassium, nitrogen, or fluoride, when available)

SOLIDS/SPECIFIC CONDUCTANCE, RATIO: Solids (residue at $180^{\circ} \mathrm{C}$, dissolved) - specific conductance (onsite); when solids, residue at $180^{\circ} \mathrm{C}$, is not available, solids, sum of constituents, is used

CATION/ANION, PERCENT DIFFERENCE:

$$
\frac{\text { sum of cations - sum of anions }}{\text { sum of cations }+ \text { sum of anions }} \times 100 \text {; }
$$

where cations and anions are expressed in milliequivalents per liter (milligrams per liter of a constituent divided by atomic or molecular weight of that constituent)

cations = potassium, calcium, magnesium, and sodium and ammonia (as N), when available 


$$
\begin{gathered}
\text { anions = alkalinity, fluoride, sulfate, and chloride and } \\
\text { nitrite plus nitrate (as N), when available }
\end{gathered}
$$

ALKALINITY: [bicarbonate $+($ carbonate $\mathrm{x} 2.03)] \times 0.8202$ $(6.0-\mathrm{pH})$

CARBON DIOXIDE: (bicarbonate $\times 1.60$ ) $\times 10$

HARDNESS: [(calcium x 0.0499) + (magnesium x 0.08226)] $\times 50.05$

NITROGEN, ORGANIC DISSOLVED: [ (nitrogen, ammonia + organic as N) - (nitrogen, ammonia as N)]

NITROGEN, AMMONIA AS $\mathrm{NH}_{4}$ : (nitrogen, ammonia as $\mathrm{N}$ ) $\times 1.288$

Notations:

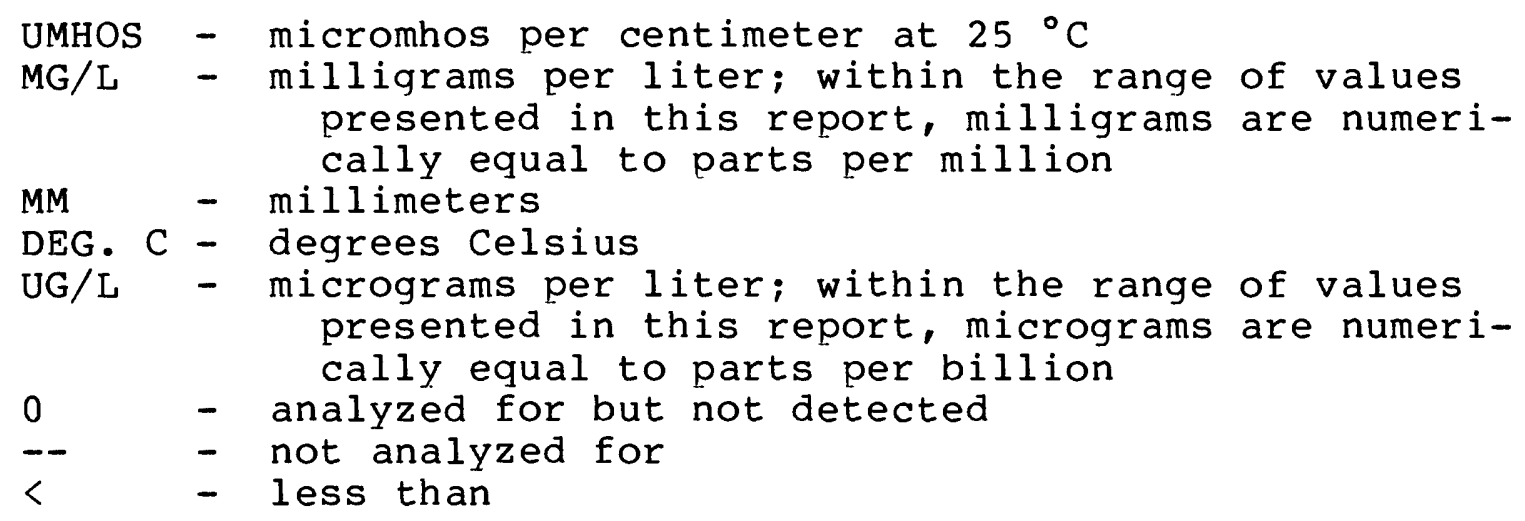


Table 3.--Ground-water quality data, 1984 and 1985

\begin{tabular}{|c|c|c|c|c|c|c|c|c|c|c|c|}
\hline $\begin{array}{l}\text { WELL } \\
\text { NUM- } \\
\text { BER }\end{array}$ & $\begin{array}{l}\text { WELL } \\
\text { LOCA- } \\
\text { TION }\end{array}$ & $\begin{array}{c}\text { DATE } \\
\text { OF } \\
\text { SAMPLE }\end{array}$ & TIME & $\begin{array}{l}\text { SPE- } \\
\text { CIFIC } \\
\text { CONDUC- } \\
\text { TANCE* } \\
\text { (UMHOS) }\end{array}$ & $\begin{array}{c}\text { SPE- } \\
\text { CIFIC } \\
\text { CONDUC- } \\
\text { TANCE, } \\
\text { LAB } \\
\text { (UMHOS) }\end{array}$ & $\begin{array}{l}\text { SOLIDS, } \\
\text { SUM OF } \\
\text { CONSTI- } \\
\text { TUENTS, } \\
\text { DIS- } \\
\text { SOLVED } \\
(M G / L)\end{array}$ & $\begin{array}{l}\text { SOLIDS, } \\
\text { RESIDUE } \\
\text { AT } 180 \\
\text { DEG. C, } \\
\text { DIS- } \\
\text { SOLVED } \\
\text { (MG/L) }\end{array}$ & $\begin{array}{l}\text { SOLIDS/ } \\
\text { SPE- } \\
\text { CIFIC } \\
\text { CONDUC- } \\
\text { TANCE, } \\
\text { RATIO }\end{array}$ & $\begin{array}{l}\text { CATION/ } \\
\text { ANION, } \\
\text { PERCENT } \\
\text { DIFFER- } \\
\text { ENCE }\end{array}$ & $\begin{array}{l}\text { TEMPER- } \\
\text { ATURE } \\
\text { (DEG. C) }\end{array}$ & $\begin{array}{c}\text { PH* } \\
\text { (STAN- } \\
\text { DARD } \\
\text { UNITS) }\end{array}$ \\
\hline 14 & $2 S-34 E-26 \mathrm{BCCC} I$ & $\begin{array}{l}84-03-01 \\
84-04-12 \\
84-05-25\end{array}$ & $\begin{array}{l}1520 \\
0945 \\
1620\end{array}$ & $\begin{array}{l}444 \\
471 \\
459\end{array}$ & $\begin{array}{l}470 \\
477 \\
448\end{array}$ & $\begin{array}{r}290 \\
-- \\
--\end{array}$ & $\begin{array}{r}280 \\
260 \\
--\end{array}$ & $\begin{array}{r}0.63 \\
.55 \\
--\end{array}$ & $\begin{array}{r}-4.16 \\
-- \\
--\end{array}$ & $\begin{array}{l}14.0 \\
14.5 \\
14.5\end{array}$ & $\begin{array}{l}7.4 \\
7.4 \\
7.3\end{array}$ \\
\hline $\begin{array}{l}\text { M10. } \\
\text { M11. } \\
\text { M7: } \\
\text { M8. }\end{array}$ & $\begin{array}{l}2 S-35 E-27 C C A A 1 \\
2 S-35 E-27 C C A A 2 \\
2 S-35 E-27 C C B B 1 \\
2 S-35 E-27 C C B B 2\end{array}$ & $\begin{array}{l}85-08-07 \\
85-08-07 \\
85-08-08 \\
85-08-08\end{array}$ & $\begin{array}{l}1200 \\
1315 \\
1300 \\
1400\end{array}$ & $\begin{array}{l}353 \\
325 \\
336 \\
333\end{array}$ & $\begin{array}{l}366 \\
327 \\
349 \\
344\end{array}$ & $\begin{array}{l}210 \\
190 \\
203 \\
207\end{array}$ & $\begin{array}{l}211 \\
185 \\
207 \\
191\end{array}$ & $\begin{array}{l}.60 \\
.57 \\
.61 \\
.57\end{array}$ & $\begin{array}{r}3.20 \\
-3.97 \\
-.53 \\
.25\end{array}$ & $\begin{array}{l}14.5 \\
19.5 \\
12.0 \\
13.0\end{array}$ & $\begin{array}{l}7.5 \\
7.6 \\
7.6 \\
7.7\end{array}$ \\
\hline 55. & $\begin{array}{l}2 S-35 E-28 C A A D 1 \\
2 S-35 E-28 C C B B 1\end{array}$ & $\begin{array}{l}84-03-08 \\
84-04-11 \\
84-05-23 \\
84-10-10 \\
84-07-24\end{array}$ & $\begin{array}{l}1515 \\
1105 \\
1130 \\
1415 \\
1445\end{array}$ & $\begin{array}{l}393 \\
381 \\
417 \\
464 \\
495\end{array}$ & $\begin{array}{l}406 \\
-- \\
417 \\
476 \\
497\end{array}$ & $\begin{array}{r}240 \\
-- \\
-- \\
--\end{array}$ & $\begin{array}{r}236 \\
-- \\
-- \\
-- \\
--\end{array}$ & $\begin{array}{l}.60 \\
-- \\
-- \\
-- \\
--\end{array}$ & $\begin{array}{r}-1.58 \\
-- \\
-- \\
--\end{array}$ & $\begin{array}{l}13.0 \\
13.0 \\
13.0 \\
11.5 \\
12.5\end{array}$ & $\begin{array}{l}7.4 \\
7.5 \\
7.5 \\
7.4 \\
7.2\end{array}$ \\
\hline \multirow[t]{2}{*}{56.} & $2 S-35 E-28 C D D D 2$ & $\begin{array}{l}84-01-31 \\
84-03-07 \\
84-04-10 \\
84-05-22 \\
84-07-18\end{array}$ & $\begin{array}{l}1530 \\
1100 \\
1910 \\
1620 \\
1915\end{array}$ & $\begin{array}{l}502 \\
471 \\
559 \\
496 \\
529\end{array}$ & $\begin{array}{l}493 \\
482 \\
534 \\
504 \\
483\end{array}$ & $\begin{array}{r}300 \\
-- \\
-- \\
--\end{array}$ & $\begin{array}{r}279 \\
281 \\
314 \\
-- \\
--\end{array}$ & $\begin{array}{l}.56 \\
.60 \\
.56 \\
-- \\
--\end{array}$ & $\begin{array}{r}-1.95 \\
-- \\
-- \\
--\end{array}$ & $\begin{array}{r}8.5 \\
9.5 \\
9.5 \\
10.0 \\
10.0\end{array}$ & $\begin{array}{l}7.3 \\
7.7 \\
7.6 \\
7.5 \\
7.5\end{array}$ \\
\hline & & $\begin{array}{l}84-10-03 \\
85-04-04 \\
85-04-17 \\
85-06-13 \\
85-08-07\end{array}$ & $\begin{array}{l}1650 \\
1230 \\
1750 \\
0845 \\
0845\end{array}$ & $\begin{array}{l}480 \\
633 \\
658 \\
570 \\
567\end{array}$ & $\begin{array}{r}484 \\
-- \\
-- \\
590 \\
528\end{array}$ & $\begin{array}{r}290 \\
-- \\
-- \\
350 \\
340\end{array}$ & $\begin{array}{r}-- \\
-- \\
321 \\
329\end{array}$ & $\begin{array}{l}.60 \\
-- \\
-- \\
.56 \\
.58\end{array}$ & $\begin{array}{r}-3.95 \\
-= \\
-2.19 \\
-2.84\end{array}$ & $\begin{array}{l}9.5 \\
7.0 \\
8.0 \\
9.5 \\
9.5\end{array}$ & $\begin{array}{l}7.3 \\
7.5 \\
7.5 \\
7.4 \\
7.4\end{array}$ \\
\hline 58. & $2 S-35 E-28 D B A A 1$ & $\begin{array}{l}84-03-08 \\
84-04-10 \\
84-05-23 \\
84-07-19 \\
84-10-10 \\
85-06-13\end{array}$ & $\begin{array}{l}1320 \\
0830 \\
1040 \\
0915 \\
1100 \\
1530\end{array}$ & $\begin{array}{l}354 \\
368 \\
363 \\
469 \\
443 \\
378\end{array}$ & $\begin{array}{r}364 \\
375 \\
372 \\
-- \\
454 \\
398\end{array}$ & $\begin{array}{r}210 \\
-- \\
-- \\
-- \\
260 \\
240\end{array}$ & $\begin{array}{r}213 \\
216 \\
-- \\
-- \\
267 \\
230\end{array}$ & $\begin{array}{l}.60 \\
.59 \\
-- \\
-- \\
.60 \\
.61\end{array}$ & $\begin{array}{r}-2.10 \\
-- \\
-- \\
-7 \\
-1.73 \\
.50\end{array}$ & $\begin{array}{l}13.5 \\
13.0 \\
12.0 \\
11.5 \\
14.5 \\
12.0\end{array}$ & $\begin{array}{l}7.6 \\
7.4 \\
7.4 \\
7.3 \\
7.3 \\
7.4\end{array}$ \\
\hline 59. & $2 S-35 E-28 D B C D 1$ & $\begin{array}{l}84-03-06 \\
84-04-10 \\
84-05-22 \\
84-07-19 \\
84-10-10\end{array}$ & $\begin{array}{l}1700 \\
0930 \\
1530 \\
1015 \\
1000\end{array}$ & $\begin{array}{l}530 \\
559 \\
645 \\
523 \\
496\end{array}$ & $\begin{array}{l}536 \\
-- \\
645 \\
515 \\
508\end{array}$ & $\begin{array}{c}320 \\
-- \\
-- \\
300\end{array}$ & $\begin{array}{c}313 \\
-- \\
-- \\
277\end{array}$ & $\begin{array}{l}.59 \\
-- \\
-- \\
-\overline{.56}\end{array}$ & $\begin{array}{r}-.45 \\
-- \\
-\overline{-} \\
-1.12\end{array}$ & $\begin{array}{l}12.5 \\
12.5 \\
12.5 \\
13.0 \\
13.0\end{array}$ & $\begin{array}{l}7.6 \\
7.6 \\
7.5 \\
7.4 \\
7.4\end{array}$ \\
\hline M5. & $2 S-35 E-28 D C D C 1$ & $\begin{array}{l}85-04-04 \\
85-04-17 \\
85-06-13 \\
85-07-24 \\
85-08-07\end{array}$ & $\begin{array}{l}1500 \\
1550 \\
1430 \\
1345 \\
1620\end{array}$ & $\begin{array}{l}495 \\
559 \\
542 \\
565 \\
418\end{array}$ & $\begin{array}{r}-- \\
5 \overline{64} \\
\overline{424}\end{array}$ & $\begin{array}{r}-- \\
330 \\
260\end{array}$ & $\begin{array}{r}-- \\
317 \\
249\end{array}$ & $\begin{array}{l}-- \\
.- \\
.58 \\
. \overline{-60}\end{array}$ & $\begin{array}{r}-- \\
-. \overline{28} \\
-. \overline{70}\end{array}$ & $\begin{array}{r}13.0 \\
11.5 \\
13.0 \\
12.5 \\
9.5\end{array}$ & $\begin{array}{l}7.5 \\
7.6 \\
7.4 \\
7.4 \\
7.2\end{array}$ \\
\hline
\end{tabular}


Table 3.--Ground-water quality data, 1984 and 1985--Continued

\begin{tabular}{|c|c|c|c|c|c|c|c|c|c|c|c|c|}
\hline $\begin{array}{c}\text { DATE } \\
\text { OF } \\
\text { SAMPLE }\end{array}$ & $\begin{array}{c}\text { OXYGEN*, } \\
\text { DIS- } \\
\text { SOLVED } \\
\text { (MG/L) }\end{array}$ & $\begin{array}{l}\text { BARO- } \\
\text { METRIC } \\
\text { PRES- } \\
\text { SURE* } \\
\text { (MM } \\
\text { OF HG) }\end{array}$ & $\begin{array}{c}\text { ALKA- } \\
\text { LINITY* } \\
\text { (MG/L } \\
\text { AS } \\
\text { (ACO3) }\end{array}$ & $\begin{array}{c}\text { BICAR- } \\
\text { BONATE* } \\
\text { FET-FLD } \\
\text { (MG/L AS } \\
\text { HCO3) }\end{array}$ & $\begin{array}{c}\text { CAR- } \\
\text { BONATE* } \\
\text { FET-FLD } \\
\text { (MG/L } \\
\text { AS CO3) }\end{array}$ & $\begin{array}{l}\text { CARBON } \\
\text { DIOXIDE, } \\
\text { DIS- } \\
\text { SOLVED } \\
\text { (MG/L } \\
\text { AS CO2) }\end{array}$ & $\begin{array}{l}\text { HARD- } \\
\text { NESS } \\
\text { (MG/L } \\
\text { AS } \\
\text { CACO3) }\end{array}$ & $\begin{array}{l}\text { CALCIUM, } \\
\text { DIS- } \\
\text { SOLVED } \\
\text { (MG/L } \\
\text { AS CA) }\end{array}$ & $\begin{array}{l}\text { MAGNE- } \\
\text { S IUM, } \\
\text { DIS- } \\
\text { SOLVED } \\
\text { (MG/L } \\
\text { AS MG) }\end{array}$ & $\begin{array}{l}\text { SODIUM, } \\
\text { DIS- } \\
\text { SOLVED } \\
\text { (MG/L } \\
\text { AS NA) }\end{array}$ & $\begin{array}{l}\text { POTAS- } \\
\text { SIUM, } \\
\text { DIS- } \\
\text { SOLVED } \\
\text { (MG/L } \\
\text { AS K) }\end{array}$ & $\begin{array}{l}\text { FLUO- } \\
\text { RIDE, } \\
\text { DIS- } \\
\text { SOLVED } \\
\text { (MG/L } \\
\text { AS F) }\end{array}$ \\
\hline $\begin{array}{l}84-03-01 \\
84-04-12 \\
84-05-25\end{array}$ & $\begin{array}{l}-\overline{-} \\
2.8 \\
3.1\end{array}$ & $\begin{array}{l}-\overline{2} \\
643 \\
642\end{array}$ & $\begin{array}{l}230 \\
220 \\
210\end{array}$ & $\begin{array}{l}280 \\
270 \\
260\end{array}$ & $\begin{array}{l}0 \\
0 \\
0\end{array}$ & $\begin{array}{l}18 \\
17 \\
21\end{array}$ & $\begin{array}{r}220 \\
---\end{array}$ & $\begin{array}{l}70 \\
-- \\
--\end{array}$ & 12 & $\begin{array}{r}9.7 \\
---\end{array}$ & $\begin{array}{l}3.5 \\
3.2 \\
2.9\end{array}$ & $\begin{array}{c}0.70 \\
-- \\
--\end{array}$ \\
\hline $\begin{array}{l}85-08-07 \\
85-08-07 \\
85-08-08 \\
85-08-08\end{array}$ & $\begin{array}{l}4.9 \\
1.3 \\
2.8 \\
2.5\end{array}$ & $\begin{array}{l}641 \\
640 \\
640 \\
641\end{array}$ & $\begin{array}{l}140 \\
140 \\
140 \\
140\end{array}$ & $\begin{array}{l}170 \\
170 \\
170 \\
170\end{array}$ & $\begin{array}{l}0 \\
0 \\
0 \\
0\end{array}$ & $\begin{array}{l}8.5 \\
6.8 \\
6.8 \\
5.4\end{array}$ & $\begin{array}{l}170 \\
140 \\
150 \\
160\end{array}$ & $\begin{array}{l}49 \\
40 \\
44 \\
44\end{array}$ & $\begin{array}{l}11 \\
9.9 \\
10 \\
11\end{array}$ & $\begin{array}{l}12 \\
9.7 \\
11 \\
11\end{array}$ & $\begin{array}{l}2.4 \\
2.9 \\
2.2 \\
2.4\end{array}$ & $\begin{array}{l}.70 \\
.50 \\
.70 \\
.70\end{array}$ \\
\hline $\begin{array}{l}84-03-08 \\
84-04-11 \\
84-05-23 \\
84-10-10 \\
84-07-24\end{array}$ & $\begin{array}{l}6.4 \\
5.9 \\
7.4 \\
5.0 \\
3.7\end{array}$ & $\begin{array}{l}647 \\
-- \\
641 \\
639 \\
647\end{array}$ & $\begin{array}{l}160 \\
160 \\
160 \\
210 \\
250\end{array}$ & $\begin{array}{l}200 \\
200 \\
200 \\
260 \\
310\end{array}$ & $\begin{array}{l}0 \\
0 \\
0 \\
0 \\
0\end{array}$ & $\begin{array}{l}13 \\
10 \\
10 \\
16 \\
31\end{array}$ & $\begin{array}{c}180 \\
-- \\
-- \\
--\end{array}$ & $\begin{array}{l}54 \\
-- \\
-- \\
--\end{array}$ & $11=$ & $\begin{array}{l}11 \\
=- \\
=-\end{array}$ & $\begin{array}{r}2.2 \\
-- \\
2.2 \\
2.2 \\
--\end{array}$ & $\begin{array}{l}.70 \\
-- \\
-- \\
--\end{array}$ \\
\hline $\begin{array}{l}84-01-31 \\
84-03-07 \\
84-04-10 \\
84-05-22 \\
84-07-18\end{array}$ & $\begin{array}{l}<.1 \\
.1 \\
.1 \\
.2 \\
.4\end{array}$ & $\begin{array}{l}-\overline{6} \\
635 \\
645 \\
640\end{array}$ & $\begin{array}{l}220 \\
210 \\
280 \\
210 \\
260\end{array}$ & $\begin{array}{l}270 \\
260 \\
340 \\
260 \\
310\end{array}$ & $\begin{array}{l}0 \\
0 \\
0 \\
0 \\
0\end{array}$ & $\begin{array}{l}22 \\
8.2 \\
14 \\
13 \\
16\end{array}$ & $\begin{array}{r}220 \\
-- \\
-- \\
--\end{array}$ & $\begin{array}{l}59 \\
-- \\
-- \\
--\end{array}$ & $\begin{array}{c}18 \\
-- \\
-- \\
--\end{array}$ & $\begin{array}{c}15 \\
-- \\
-- \\
--\end{array}$ & $\begin{array}{l}4.5 \\
-.-1 \\
3.1 \\
4.9\end{array}$ & $\begin{array}{l}.90 \\
-- \\
-- \\
--\end{array}$ \\
\hline $\begin{array}{l}84-10-03 \\
85-04-04 \\
85-04-17 \\
85-06-13 \\
85-08-07\end{array}$ & $\begin{array}{l}.2 \\
.1 \\
.2 \\
.1 \\
.4\end{array}$ & $\begin{array}{l}640 \\
641 \\
635 \\
642 \\
641\end{array}$ & $\begin{array}{l}220 \\
300 \\
320 \\
290 \\
280\end{array}$ & $\begin{array}{l}270 \\
370 \\
390 \\
350 \\
340\end{array}$ & $\begin{array}{l}0 \\
0 \\
0 \\
0 \\
0\end{array}$ & $\begin{array}{l}22 \\
19 \\
20 \\
22 \\
22\end{array}$ & $\begin{array}{r}200 \\
-- \\
270 \\
260\end{array}$ & $\begin{array}{l}52 \\
-- \\
-\overline{72} \\
68\end{array}$ & $\begin{array}{l}18 \\
22 \\
21\end{array}$ & $\begin{array}{l}14 \\
17 \\
16\end{array}$ & $\begin{array}{l}4.6 \\
5.2 \\
5.9 \\
5.3 \\
5.4\end{array}$ & $\begin{array}{l}.90 \\
-- \\
.0 \\
.80 \\
.80\end{array}$ \\
\hline $\begin{array}{l}84-03-08 \\
84-04-10 \\
84-05-23 \\
84-07-19 \\
84-10-10 \\
85-06-13\end{array}$ & $\begin{array}{l}7.0 \\
8.0 \\
8.0 \\
6.3 \\
5.8 \\
3.1\end{array}$ & $\begin{array}{l}648 \\
639 \\
641 \\
645 \\
640 \\
640\end{array}$ & $\begin{array}{l}140 \\
120 \\
150 \\
220 \\
200 \\
170\end{array}$ & $\begin{array}{l}170 \\
150 \\
180 \\
270 \\
240 \\
210\end{array}$ & $\begin{array}{l}0 \\
0 \\
0 \\
0 \\
0 \\
0\end{array}$ & $\begin{array}{l}6.8 \\
9.5 \\
11 \\
22 \\
19 \\
13\end{array}$ & $\begin{array}{r}150 \\
-- \\
-- \\
210 \\
190\end{array}$ & $\begin{array}{l}45 \\
-- \\
-- \\
-- \\
65 \\
58\end{array}$ & $\begin{array}{l}10 \\
11 \\
11 \\
11\end{array}$ & 11 & $\begin{array}{l}2.8 \\
2.3 \\
2.1 \\
2.1 \\
1.5 \\
2.6\end{array}$ & $\begin{array}{l}.80 \\
-- \\
-- \\
.0 \\
.60 \\
.60\end{array}$ \\
\hline $\begin{array}{l}84-03-06 \\
84-04-10 \\
84-05-22 \\
84-07-19 \\
84-10-10\end{array}$ & $\begin{array}{r}1.0 \\
.3 \\
.2 \\
.5 \\
.1\end{array}$ & $\begin{array}{l}646 \\
-- \\
645 \\
645 \\
640\end{array}$ & $\begin{array}{l}240 \\
260 \\
320 \\
260 \\
240\end{array}$ & $\begin{array}{l}290 \\
310 \\
390 \\
320 \\
290\end{array}$ & $\begin{array}{l}0 \\
0 \\
0 \\
0 \\
0\end{array}$ & $\begin{array}{l}12 \\
12 \\
20 \\
20 \\
18\end{array}$ & $\begin{array}{r}260 \\
-- \\
\overline{-} \\
240\end{array}$ & $\begin{array}{l}73 \\
\overline{--} \\
\overline{70}\end{array}$ & 19 & 15 & $\begin{array}{l}2.6 \\
-- \\
3.0 \\
2.6 \\
2.4\end{array}$ & $\begin{array}{l}.90 \\
-- \\
\overline{-} \\
.80\end{array}$ \\
\hline $\begin{array}{l}85-04-04 \\
85-04-17 \\
85-06-13 \\
85-07-24 \\
85-08-07\end{array}$ & $\begin{array}{l}.7 \\
.2 \\
.4 \\
.8 \\
.7\end{array}$ & $\begin{array}{l}641 \\
635 \\
641 \\
642 \\
641\end{array}$ & $\begin{array}{l}200 \\
260 \\
260 \\
260 \\
190\end{array}$ & $\begin{array}{l}270 \\
310 \\
320 \\
320 \\
230\end{array}$ & $\begin{array}{l}0 \\
0 \\
0 \\
0 \\
0\end{array}$ & $\begin{array}{l}14 \\
12 \\
20 \\
20 \\
23\end{array}$ & $\begin{array}{l}-- \\
270 \\
200\end{array}$ & $\begin{array}{l}-- \\
\overline{79} \\
-\overline{57}\end{array}$ & $18^{--}$ & $14^{--}$ & $\begin{array}{l}2.8 \\
2.7 \\
2.5 \\
-.5 \\
2.5\end{array}$ & $\begin{array}{l}-- \\
\overline{80} \\
.80\end{array}$ \\
\hline
\end{tabular}


Table 3.--Ground-water quality data, 1984 and 1985--Continued

\begin{tabular}{|c|c|c|c|c|c|c|c|c|c|c|c|c|}
\hline $\begin{array}{c}\text { DATE } \\
\text { OF } \\
\text { SAMPLE }\end{array}$ & $\begin{array}{l}\text { CHLO- } \\
\text { RIDE, } \\
\text { DIS- } \\
\text { SOLVED } \\
\text { (MG/L } \\
\text { AS CL) }\end{array}$ & $\begin{array}{c}\text { SULFATE, } \\
\text { DIS- } \\
\text { SOLVED } \\
\text { (MG/L } \\
\text { AS SO4) }\end{array}$ & $\begin{array}{l}\text { SILICA, } \\
\text { DIS- } \\
\text { SOLVED } \\
\text { (MG/L } \\
\text { AS } \\
\text { SIO2) }\end{array}$ & $\begin{array}{l}\text { CARBON, } \\
\text { ORGANIC, } \\
\text { DISSOLVED } \\
\text { (MG/L } \\
\text { AS C) }\end{array}$ & $\begin{array}{l}\text { METHY- } \\
\text { LENE } \\
\text { BLUE } \\
\text { ACTIVE } \\
\text { SUB- } \\
\text { STANCE } \\
\text { (MG/L) }\end{array}$ & $\begin{array}{c}\text { NITRO- } \\
\text { GEN, } \\
\text { NO2+NO3 } \\
\text { DIS- } \\
\text { SOLVED } \\
\text { (MG/L } \\
\text { AS N) }\end{array}$ & $\begin{array}{l}\text { NITRO- } \\
\text { GEN, } \\
\text { AMMONIA } \\
\text { DIS- } \\
\text { SOLVED } \\
\text { (MG/L } \\
\text { AS N) }\end{array}$ & $\begin{array}{l}\text { NITRO- } \\
\text { GEN,AM- } \\
\text { MONIA + } \\
\text { ORGANIC } \\
\text { DISSOLVED } \\
\text { (MG/L } \\
\text { AS N) }\end{array}$ & $\begin{array}{l}\text { NITRO- } \\
\text { GEN, } \\
\text { ORGANIC } \\
\text { DIS- } \\
\text { SOLVED } \\
\text { (MG/L } \\
\text { AS N) }\end{array}$ & $\begin{array}{l}\text { NITRO- } \\
\text { GEN, } \\
\text { AMMONIA, } \\
\text { DIS- } \\
\text { SOLVED } \\
\text { (MG/L } \\
\text { AS NH4) }\end{array}$ & $\begin{array}{l}\text { PHOS- } \\
\text { PHORUS, } \\
\text { DIS- } \\
\text { SOLVED } \\
\text { (MG/L } \\
\text { AS P) }\end{array}$ & $\begin{array}{c}\text { IRON, } \\
\text { DIS- } \\
\text { SOLVED } \\
\text { (UG/L } \\
\text { AS FE) }\end{array}$ \\
\hline $\begin{array}{l}84-03-01 \\
84-04-12 \\
84-05-25\end{array}$ & $\begin{array}{l}7.9 \\
-- \\
--\end{array}$ & $\begin{array}{l}26 \\
25 \\
--\end{array}$ & $\begin{array}{l}23 \\
-- \\
--\end{array}$ & $\begin{array}{c}1.3 \\
.90 \\
.80\end{array}$ & $\begin{array}{l}-- \\
-- \\
--\end{array}$ & $\begin{array}{r}0.41 \\
.52 \\
.60\end{array}$ & $\begin{array}{r}0.050 \\
.020 \\
<.010\end{array}$ & $\begin{array}{r}--- \\
0.30 \\
.60\end{array}$ & $\begin{array}{c}-\overline{-} \\
0.28 \\
--\end{array}$ & $\begin{array}{r}0.06 \\
.03 \\
--\end{array}$ & $\begin{array}{r}-- \\
0.060\end{array}$ & $\begin{array}{r}7 \\
8 \\
10\end{array}$ \\
\hline $\begin{array}{l}85-08-07 \\
85-08-07 \\
85-08-08 \\
85-08-08\end{array}$ & $\begin{array}{l}8.5 \\
7.7 \\
7.4 \\
8.1\end{array}$ & $\begin{array}{l}28 \\
25 \\
26 \\
26\end{array}$ & $\begin{array}{l}19 \\
15 \\
17 \\
18\end{array}$ & $\begin{array}{l}1.3 \\
1.2 \\
1.0 \\
1.0\end{array}$ & $\begin{array}{l}-- \\
-- \\
-- \\
--\end{array}$ & $\begin{array}{l}.48 \\
.31 \\
.13 \\
.28\end{array}$ & $\begin{array}{l}.020 \\
.040 \\
.030 \\
.020\end{array}$ & $\begin{array}{r}<.20 \\
.30 \\
.10 \\
<.10\end{array}$ & $\begin{array}{l}-- \\
.26 \\
.07 \\
--\end{array}$ & $\begin{array}{l}.03 \\
.05 \\
.04 \\
.03\end{array}$ & $\begin{array}{l}-- \\
-- \\
-- \\
--\end{array}$ & $\begin{array}{l}24 \\
52 \\
36 \\
19\end{array}$ \\
\hline $\begin{array}{l}84-03-08 \\
84-04-11 \\
84-05-23 \\
84-10-10 \\
84-07-24\end{array}$ & $11=$ & $\begin{array}{l}32 \\
-- \\
-- \\
-- \\
--\end{array}$ & $\begin{array}{l}17 \\
-- \\
-- \\
-- \\
--\end{array}$ & $\begin{array}{l}.80 \\
.60 \\
.- \\
--\end{array}$ & $\begin{array}{l}-- \\
-- \\
-- \\
--\end{array}$ & $\begin{array}{c}.64 \\
-2 \\
1.7 \\
1.5 \\
.92\end{array}$ & $\begin{array}{r}.030 \\
-- \\
<.010 \\
<.010 \\
<.010\end{array}$ & $\begin{array}{l}-- \\
-\overline{-} \\
.30 \\
.20 \\
.50\end{array}$ & $\begin{array}{l}-- \\
-- \\
-- \\
--\end{array}$ & $\begin{array}{l}.04 \\
-- \\
-- \\
-- \\
--\end{array}$ & $\begin{array}{r}-- \\
.060 \\
-- \\
--\end{array}$ & $\begin{array}{l}<3 \\
-- \\
<3 \\
<3 \\
--\end{array}$ \\
\hline $\begin{array}{l}84-01-31 \\
84-03-07 \\
84-04-10 \\
84-05-22 \\
84-07-18\end{array}$ & $\begin{array}{r}13 \\
=- \\
=- \\
--\end{array}$ & $\begin{array}{l}28 \\
-- \\
16 \\
-- \\
--\end{array}$ & $\begin{array}{l}24 \\
-- \\
-- \\
-- \\
--\end{array}$ & $\begin{array}{l}.90 \\
1.9 \\
.90 \\
1.5\end{array}$ & $\begin{array}{r}-- \\
\overline{-0} \\
\overline{--} \\
--\end{array}$ & $\begin{array}{l}.10 \\
<.10 \\
<.10 \\
<.10 \\
<.10\end{array}$ & $\begin{array}{l}.-- \\
.910 \\
.760 \\
.390 \\
.980\end{array}$ & $\begin{array}{l}-- \\
1.3 \\
.90 \\
1.3\end{array}$ & $\begin{array}{l}-- \\
-\overline{-} \\
.54 \\
.51 \\
.32\end{array}$ & $\begin{array}{l}1.2 \\
.98 \\
.50 \\
1.3\end{array}$ & $\begin{array}{r}-- \\
-- \\
-- \\
.050 \\
<.010\end{array}$ & $\begin{array}{r}1800 \\
1700 \\
1400 \\
460 \\
2000\end{array}$ \\
\hline $\begin{array}{l}84-10-03 \\
85-04-04 \\
85-04-17 \\
85-06-13 \\
85-08-07\end{array}$ & $\begin{array}{l}12 \\
=- \\
15 \\
14\end{array}$ & $\begin{array}{l}23 \\
-- \\
-- \\
18 \\
17\end{array}$ & $\begin{array}{l}24 \\
-- \\
-- \\
25 \\
24\end{array}$ & $\begin{array}{c}2.3 \\
-- \\
-- \\
1.9 \\
2.3\end{array}$ & $\begin{array}{l}-- \\
-- \\
-- \\
--\end{array}$ & $\begin{array}{r}<.10 \\
-1 \\
<.10 \\
<.10 \\
.13\end{array}$ & $\begin{array}{c}.810 \\
-- \\
1.20 \\
1.00 \\
1.20\end{array}$ & $\begin{array}{c}1.0 \\
1.7 \\
1.4\end{array}$ & $\begin{array}{r}.19 \\
-- \\
.50 \\
-- \\
.20\end{array}$ & $\begin{array}{l}1.0 \\
-- \\
1.5 \\
1.3 \\
1.5\end{array}$ & $\begin{array}{r}.020 \\
-- \\
-- \\
--\end{array}$ & $\begin{array}{l}1700 \\
1900 \\
3000 \\
2300 \\
2200\end{array}$ \\
\hline $\begin{array}{l}84-03-08 \\
84-04-10 \\
84-05-23 \\
84-07-19 \\
84-10-10 \\
85-06-13\end{array}$ & $\begin{array}{r}11 \\
-- \\
-- \\
-- \\
9.6 \\
7.5\end{array}$ & $\begin{array}{l}28 \\
30 \\
-- \\
-- \\
24 \\
29\end{array}$ & $\begin{array}{l}16 \\
-- \\
-- \\
-- \\
17 \\
16\end{array}$ & $\begin{array}{l}.80 \\
.60 \\
.90 \\
1.1 \\
2.8 \\
2.2\end{array}$ & $\begin{array}{l}-- \\
-- \\
-- \\
-- \\
-- \\
--\end{array}$ & $\begin{array}{r}.44 \\
.60 \\
.70 \\
1.6 \\
.44 \\
.22\end{array}$ & $\begin{array}{r}.020 \\
.050 \\
<.010 \\
.030 \\
<.010 \\
.020\end{array}$ & $\begin{array}{l}-- \\
.30 \\
.70 \\
.40 \\
.30 \\
--\end{array}$ & $\begin{array}{r}.-- \\
.25 \\
\overline{-} \\
.37 \\
-- \\
--\end{array}$ & $\begin{array}{l}.03 \\
.06 \\
. \overline{-04} \\
.03\end{array}$ & $\begin{array}{r}-- \\
-- \\
.060 \\
.030 \\
.010 \\
--\end{array}$ & $\begin{array}{l}5 \\
6 \\
4 \\
5 \\
5 \\
5\end{array}$ \\
\hline $\begin{array}{l}84-03-06 \\
84-04-10 \\
84-05-22 \\
84-07-19 \\
84-10-10\end{array}$ & $19=$ & $\begin{array}{l}28 \\
-- \\
-- \\
-\overline{24}\end{array}$ & $\begin{array}{l}21 \\
-- \\
-- \\
-- \\
21\end{array}$ & $\begin{array}{l}1.1 \\
1.2 \\
1.2 \\
2.1 \\
2.4\end{array}$ & $\begin{array}{l}-- \\
-- \\
-- \\
-- \\
--\end{array}$ & $\begin{array}{l}<.10 \\
-\overline{<} \\
<.10 \\
.48 \\
<.10\end{array}$ & $\begin{array}{r}.090 \\
-0 \\
.040 \\
.030 \\
.040\end{array}$ & $\begin{array}{r}-- \\
.- \\
.40 \\
.40 \\
<.20\end{array}$ & $\begin{array}{l}-- \\
-\overline{-} \\
.36 \\
.37 \\
--\end{array}$ & $\begin{array}{l}.12 \\
.05 \\
.05 \\
.04 \\
.05\end{array}$ & $\begin{array}{r}-- \\
-\overline{0} \\
.010 \\
<.010 \\
<.010\end{array}$ & $\begin{array}{r}53 \\
28 \\
77 \\
4 \\
29\end{array}$ \\
\hline $\begin{array}{l}85-04-04 \\
85-04-17 \\
85-06-13 \\
85-07-24 \\
85-08-07\end{array}$ & $15^{--}$ & $\begin{array}{l}-- \\
\overline{22} \\
\overline{23}\end{array}$ & $\begin{array}{l}-- \\
\overline{21} \\
\overline{23}\end{array}$ & $\begin{array}{l}-- \\
3.7 \\
1.6 \\
2.0\end{array}$ & $\begin{array}{l}-- \\
-- \\
-- \\
--\end{array}$ & $\begin{array}{r}-- \\
<.10 \\
.21 \\
.56 \\
<.10\end{array}$ & $\begin{array}{r}-0 \\
.090 \\
.040 \\
.100 \\
.440\end{array}$ & $\begin{array}{l}-- \\
.60 \\
-- \\
.30 \\
.60\end{array}$ & $\begin{array}{l}.- \\
.51 \\
-- \\
.20 \\
.16\end{array}$ & $\begin{array}{l}.- \\
.12 \\
.05 \\
.13 \\
.57\end{array}$ & $\begin{array}{l}-- \\
-- \\
-- \\
--\end{array}$ & $\begin{array}{r}25 \\
34 \\
13 \\
8 \\
950\end{array}$ \\
\hline
\end{tabular}


Table 3.--Ground-water quality data, 1984 and 1985--Continued

\begin{tabular}{|c|c|c|c|c|}
\hline $\begin{array}{c}\text { DATE } \\
\text { OF } \\
\text { SAMPLE }\end{array}$ & $\begin{array}{l}\text { IRON, } \\
\text { SUS- } \\
\text { PENDED } \\
\text { RECOV- } \\
\text { ERABLE } \\
\text { (UG/L } \\
\text { AS FE) }\end{array}$ & $\begin{array}{l}\text { IRON, } \\
\text { TOTAL } \\
\text { RECOV- } \\
\text { ERABLE } \\
\text { (UG/L } \\
\text { AS FE) }\end{array}$ & $\begin{array}{l}\text { MANGA- } \\
\text { NESE, } \\
\text { DIS- } \\
\text { SOLVED } \\
\text { (UG/L } \\
\text { AS MN) }\end{array}$ & $\begin{array}{l}\text { ZINC, } \\
\text { DIS- } \\
\text { SOLVED } \\
\text { (UG/L } \\
\text { AS } 2 N \text { ) }\end{array}$ \\
\hline $\begin{array}{l}84-03-01 \\
84-04-12 \\
84-05-25\end{array}$ & $\begin{array}{l}-- \\
--\end{array}$ & $\begin{array}{r}-\overline{10} \\
40\end{array}$ & $\begin{array}{r}<1 \\
2 \\
<10\end{array}$ & $\begin{array}{r}-- \\
95 \\
120\end{array}$ \\
\hline $\begin{array}{l}85-08-07 \\
85-08-07 \\
85-08-08 \\
85-08-08\end{array}$ & $\begin{array}{l}-- \\
-- \\
--\end{array}$ & $\begin{array}{l}-- \\
-- \\
--\end{array}$ & $\begin{array}{r}6 \\
62 \\
27 \\
6\end{array}$ & $\begin{array}{l}12 \\
38 \\
11 \\
16\end{array}$ \\
\hline $\begin{array}{l}84-03-08 \\
84-04-11 \\
84-05-23 \\
84-10-10 \\
84-07-24\end{array}$ & $\begin{array}{l}-- \\
=- \\
-- \\
--\end{array}$ & $\begin{array}{r}\overline{-} \\
220 \\
--\end{array}$ & $\begin{array}{l}<1 \\
-\frac{2}{2} \\
<1 \\
--\end{array}$ & $\begin{array}{l}-- \\
46 \\
39 \\
--\end{array}$ \\
\hline $\begin{array}{l}84-01-31 \\
84-03-07 \\
84-04-10 \\
84-05-22 \\
84-07-18\end{array}$ & $\begin{array}{l}-- \\
-0 \\
--\end{array}$ & $\begin{array}{r}-- \\
1300 \\
430 \\
2000\end{array}$ & $\begin{array}{l}2600 \\
2500 \\
2700 \\
1800 \\
2900\end{array}$ & $\begin{array}{l}-- \\
47 \\
49 \\
32\end{array}$ \\
\hline $\begin{array}{l}84-10-03 \\
85-04-04 \\
85-04-17 \\
85-06-13 \\
85-08-07\end{array}$ & $\begin{array}{l}-- \\
=- \\
=- \\
--\end{array}$ & $\begin{array}{l}= \\
=- \\
-- \\
--\end{array}$ & $\begin{array}{l}2500 \\
2900 \\
3400 \\
3000 \\
2800\end{array}$ & $\begin{array}{l}56 \\
51 \\
36 \\
47 \\
20\end{array}$ \\
\hline $\begin{array}{l}84-03-08 \\
84-04-10 \\
84-05-23 \\
84-07-19 \\
84-10-10 \\
85-06-13\end{array}$ & $\begin{array}{l}-- \\
-- \\
-- \\
-- \\
--\end{array}$ & $\begin{array}{r}-- \\
<10 \\
30 \\
<10 \\
-- \\
--\end{array}$ & $\begin{array}{r}<1 \\
2 \\
<1 \\
3 \\
<1 \\
46\end{array}$ & $\begin{array}{l}-- \\
45 \\
55 \\
39 \\
66 \\
33\end{array}$ \\
\hline $\begin{array}{l}84-03-06 \\
84-04-10 \\
84-05-22 \\
84-07-19 \\
84-10-10\end{array}$ & $\begin{array}{l}-- \\
30 \\
-- \\
--\end{array}$ & $\begin{array}{l}-- \\
\overline{80} \\
20 \\
--\end{array}$ & $\begin{array}{l}2800 \\
3100 \\
3800 \\
1700 \\
1600\end{array}$ & $\begin{array}{r}-- \\
-- \\
21 \\
<3 \\
5\end{array}$ \\
\hline $\begin{array}{l}85-04-04 \\
85-04-17 \\
85-06-13 \\
85-07-24 \\
85-08-07\end{array}$ & $\begin{array}{l}-- \\
-- \\
-- \\
--\end{array}$ & $\begin{array}{l}-- \\
-- \\
-- \\
--\end{array}$ & $\begin{array}{l}2200 \\
3000 \\
1500 \\
2200 \\
3400\end{array}$ & $\begin{array}{r}12 \\
33 \\
5 \\
320 \\
9\end{array}$ \\
\hline
\end{tabular}


Table 3.--Ground-water quality data, 1984 and 1985--Continued

\begin{tabular}{|c|c|c|c|c|c|c|c|c|c|c|c|}
\hline $\begin{array}{l}\text { WELL } \\
\text { NUM- } \\
\text { BER }\end{array}$ & $\begin{array}{l}\text { WELL } \\
\text { LOCA- } \\
\text { TION }\end{array}$ & $\begin{array}{l}\text { DATE } \\
\text { OF } \\
\text { SAMPLE }\end{array}$ & TIME & $\begin{array}{l}\text { SPE- } \\
\text { CIFIC } \\
\text { CONDUC- } \\
\text { TANCE* } \\
\text { (UMHOS) }\end{array}$ & $\begin{array}{l}\text { SPE- } \\
\text { CIFIC } \\
\text { CONDUC- } \\
\text { TANCE, } \\
\text { LAB } \\
\text { (UMHOS) }\end{array}$ & $\begin{array}{l}\text { SOLIDS, } \\
\text { SUM OF } \\
\text { CONSTI- } \\
\text { TUENTS, } \\
\text { DIS- } \\
\text { SOLVED } \\
\text { (MG/L) }\end{array}$ & $\begin{array}{l}\text { SOLIDS, } \\
\text { RESIDUE } \\
\text { AT } 180 \\
\text { DEG. C, } \\
\text { DIS- } \\
\text { SOLVED } \\
(M G / L)\end{array}$ & $\begin{array}{l}\text { SOLIDS/ } \\
\text { SPE- } \\
\text { CIFIC } \\
\text { CONDUC- } \\
\text { TANCE, } \\
\text { RATIO }\end{array}$ & $\begin{array}{l}\text { CATION/ } \\
\text { ANION, } \\
\text { PERCENT } \\
\text { DIFFER- } \\
\text { ENCE }\end{array}$ & $\begin{array}{l}\text { TEMPER- } \\
\text { ATURE^ } \\
\text { (DEG. C) }\end{array}$ & $\begin{array}{c}\text { PH* } \\
\text { (STAN- } \\
\text { DARD } \\
\text { UNITS) }\end{array}$ \\
\hline $\begin{array}{l}\text { M6. } \\
\text { M9. } \\
65 .\end{array}$ & $\begin{array}{l}2 S-35 E-28 C D C D 2 \\
2 S-35 E 28 D D D 1 \\
2 S-35 E-32 A A B 1\end{array}$ & $\begin{array}{l}85-08-07 \\
85-08-08 \\
84-05-31 \\
84-07-24 \\
84-10-12\end{array}$ & $\begin{array}{l}1500 \\
1030 \\
0940 \\
1545 \\
0940\end{array}$ & $\begin{array}{l}354 \\
318 \\
470 \\
450 \\
443\end{array}$ & $\begin{array}{r}362 \\
333 \\
453 \\
-- \\
454\end{array}$ & $\begin{array}{r}217 \\
210 \\
-- \\
-- \\
--\end{array}$ & $\begin{array}{r}210 \\
186 \\
-- \\
--\end{array}$ & $\begin{array}{r}0.59 \\
.58 \\
-- \\
-- \\
--\end{array}$ & $\begin{array}{r}0.80 \\
-5.03 \\
-2 \\
-- \\
--\end{array}$ & $\begin{array}{l}9.5 \\
13.0 \\
12.5 \\
12.5 \\
12.0\end{array}$ & $\begin{array}{l}7.3 \\
7.7 \\
7.5 \\
7.3 \\
7.4\end{array}$ \\
\hline $\begin{array}{l}66 . \\
67 .\end{array}$ & $\begin{array}{l}2 S-35 E-32 A B C C 1 \\
2 S-35 E-32 A C C 1\end{array}$ & $\begin{array}{l}84-04-12 \\
84-05-31 \\
84-07-24 \\
84-10-12 \\
84-05-31\end{array}$ & $\begin{array}{l}1915 \\
1215 \\
1845 \\
1730 \\
2000\end{array}$ & $\begin{array}{l}456 \\
491 \\
450 \\
437 \\
473\end{array}$ & $\begin{array}{l}--- \\
493 \\
453 \\
448 \\
465\end{array}$ & $\begin{array}{l}-- \\
=- \\
=- \\
--\end{array}$ & $\begin{array}{l}-- \\
-- \\
=- \\
--\end{array}$ & $\begin{array}{l}-- \\
-- \\
=- \\
--\end{array}$ & $\begin{array}{l}-- \\
=- \\
-- \\
--\end{array}$ & $\begin{array}{l}10.5 \\
10.5 \\
10.5 \\
10.5 \\
11.5\end{array}$ & $\begin{array}{l}7.3 \\
7.4 \\
7.3 \\
7.3 \\
7.4\end{array}$ \\
\hline $\begin{array}{l}68 . \\
69 .\end{array}$ & $\begin{array}{l}2 S-35 E-32 A C D D 1 \\
2 S-35 E-32 A C D D 2\end{array}$ & $\begin{array}{l}84-07-25 \\
84-10-11 \\
84-03-02 \\
84-03-01 \\
84-04-11\end{array}$ & $\begin{array}{l}0945 \\
1745 \\
0945 \\
1645 \\
1450\end{array}$ & $\begin{array}{l}506 \\
523 \\
494 \\
456 \\
517\end{array}$ & $\begin{array}{l}509 \\
534 \\
500 \\
507 \\
511\end{array}$ & $\begin{array}{r}-- \\
300 \\
300 \\
--\end{array}$ & $\begin{array}{r}-- \\
299 \\
304 \\
306\end{array}$ & $\begin{array}{l}-- \\
.61 \\
.67 \\
.59\end{array}$ & $\begin{array}{r}-- \\
-1.35 \\
-1.67 \\
--\end{array}$ & $\begin{array}{l}12.0 \\
12.0 \\
11.0 \\
11.5 \\
12.5\end{array}$ & $\begin{array}{l}7.3 \\
7.3 \\
7.4 \\
7.3 \\
7.3\end{array}$ \\
\hline 72. & $2 S-35 E-32 A D D C 1$ & $\begin{array}{l}84-05-24 \\
84-07-25 \\
84-10-12 \\
84-02-02 \\
84-03-06\end{array}$ & $\begin{array}{l}1720 \\
1045 \\
1120 \\
1445 \\
1330\end{array}$ & $\begin{array}{l}432 \\
475 \\
502 \\
553 \\
533\end{array}$ & $\begin{array}{l}432 \\
476 \\
515 \\
549 \\
545\end{array}$ & $\begin{array}{l}-- \\
\overline{--} \\
330\end{array}$ & $\begin{array}{r}-- \\
\overline{--} \\
319 \\
313\end{array}$ & $\begin{array}{l}-- \\
-- \\
.- \\
.58 \\
.59\end{array}$ & $\begin{array}{r}-- \\
\overline{--} \\
-1.03 \\
--\end{array}$ & $\begin{array}{l}12.5 \\
12.5 \\
12.0 \\
10.0 \\
10.0\end{array}$ & $\begin{array}{l}7.3 \\
7.4 \\
7.3 \\
7.1 \\
7.4\end{array}$ \\
\hline 73 & $2 S-35 E-32 A D D D 1$ & $\begin{array}{l}84-04-11 \\
84-05-22 \\
84-07-24 \\
84-10-10 \\
84-03-06\end{array}$ & $\begin{array}{l}1305 \\
1745 \\
1040 \\
1215 \\
1500\end{array}$ & $\begin{array}{l}635 \\
527 \\
651 \\
494 \\
523\end{array}$ & $\begin{array}{l}626 \\
530 \\
648 \\
508 \\
536\end{array}$ & $\begin{array}{l}-- \\
\overline{--} \\
290 \\
--\end{array}$ & $\begin{array}{r}361 \\
-- \\
276 \\
--\end{array}$ & $\begin{array}{l}.57 \\
-- \\
.- \\
.56 \\
--\end{array}$ & $\begin{array}{r}-- \\
-\overline{-} \\
-2.06 \\
--\end{array}$ & $\begin{array}{l}10.5 \\
10.5 \\
10.5 \\
10.5 \\
10.5\end{array}$ & $\begin{array}{l}7.4 \\
7.2 \\
7.2 \\
7.4 \\
7.4\end{array}$ \\
\hline $\begin{array}{l}74 . \\
76 .\end{array}$ & $\begin{array}{l}2 S-35 E-32 B A A 1 \\
2 S-35 E-32 C A D 1\end{array}$ & $\begin{array}{l}84-05-31 \\
84-07-24 \\
84-10-12 \\
84-05-31 \\
84-07-25\end{array}$ & $\begin{array}{l}1730 \\
1645 \\
1020 \\
1400 \\
1245\end{array}$ & $\begin{array}{l}494 \\
509 \\
463 \\
418 \\
406\end{array}$ & $\begin{array}{l}483 \\
491 \\
475 \\
414 \\
409\end{array}$ & $\begin{array}{l}-- \\
=- \\
-- \\
--\end{array}$ & $\begin{array}{l}-- \\
=- \\
=- \\
--\end{array}$ & $\begin{array}{l}-- \\
-- \\
-- \\
--\end{array}$ & $\begin{array}{l}-- \\
=- \\
=- \\
--\end{array}$ & $\begin{array}{l}12.5 \\
12.5 \\
12.0 \\
12.0 \\
12.5\end{array}$ & $\begin{array}{l}7.4 \\
7.3 \\
7.3 \\
7.6 \\
7.5\end{array}$ \\
\hline 79. & $2 S-35 E-32 C C D D 1$ & $\begin{array}{l}84-10-11 \\
84-03-01 \\
84-04-12 \\
84-05-23 \\
84-07-25\end{array}$ & $\begin{array}{l}1215 \\
1445 \\
1100 \\
1440 \\
1330\end{array}$ & $\begin{array}{l}441 \\
422 \\
518 \\
568 \\
520\end{array}$ & $\begin{array}{l}453 \\
432 \\
510 \\
579 \\
518\end{array}$ & $\begin{array}{r}-- \\
270 \\
-- \\
--\end{array}$ & $\begin{array}{r}-- \\
307 \\
-- \\
--\end{array}$ & $\begin{array}{l}.-- \\
.64 \\
.59 \\
-- \\
--\end{array}$ & $\begin{array}{r}-\overline{-} \\
-4.21 \\
-- \\
--\end{array}$ & $\begin{array}{l}11.5 \\
12.0 \\
11.5 \\
13.0 \\
12.5\end{array}$ & $\begin{array}{l}7.4 \\
7.4 \\
7.2 \\
7.1 \\
7.2\end{array}$ \\
\hline 82. & $2 S-35 E-32 C D D C 2$ & $\begin{array}{l}84-10-11 \\
84-02-28 \\
84-04-10 \\
84-05-22 \\
84-07-25\end{array}$ & $\begin{array}{l}1030 \\
1715 \\
1610 \\
1350 \\
1130\end{array}$ & $\begin{array}{l}480 \\
369 \\
388 \\
456 \\
402\end{array}$ & $\begin{array}{l}493 \\
372 \\
396 \\
449 \\
402\end{array}$ & $\begin{array}{r}230 \\
230 \\
-- \\
--\end{array}$ & $\begin{array}{r}-- \\
220 \\
243 \\
-- \\
--\end{array}$ & $\begin{array}{l}.-- \\
.60 \\
.63 \\
-- \\
--\end{array}$ & $\begin{array}{r}--- \\
-.25 \\
-3.75 \\
-- \\
--\end{array}$ & $\begin{array}{l}13.5 \\
13.0 \\
12.0 \\
11.0 \\
11.0\end{array}$ & $\begin{array}{l}7.2 \\
7.5 \\
7.5 \\
7.3 \\
7.4\end{array}$ \\
\hline
\end{tabular}


Table 3.--Ground-water quality data, 1984 and $1985--C o n t i n u e d$

\begin{tabular}{|c|c|c|c|c|c|c|c|c|c|c|c|c|}
\hline $\begin{array}{c}\text { DATE } \\
\text { OF } \\
\text { SAMPLE }\end{array}$ & $\begin{array}{l}\text { OXYGEN*, } \\
\text { DIS- } \\
\text { SOLVED } \\
\text { (MG/L) }\end{array}$ & $\begin{array}{l}\text { BARO- } \\
\text { METRIC } \\
\text { PRES- } \\
\text { SURE* } \\
\text { (MM } \\
\text { OF HG) }\end{array}$ & $\begin{array}{l}\text { ALKA- } \\
\text { LINITY* } \\
\text { (MG/L } \\
\text { AS } \\
\text { CACO3) }\end{array}$ & $\begin{array}{l}\text { BICAR- } \\
\text { BONATE* } \\
\text { FET-FLD } \\
\text { (MG/L AS } \\
\text { HCO3) }\end{array}$ & $\begin{array}{c}\text { CAR- } \\
\text { BONATE* } \\
\text { FET-FLD } \\
\text { (MG/L } \\
\text { AS CO3) }\end{array}$ & $\begin{array}{c}\text { CARBON } \\
\text { DIOXIDE, } \\
\text { DIS- } \\
\text { SOLVED } \\
\text { (MG/L } \\
\text { AS CO2) }\end{array}$ & $\begin{array}{l}\text { HARD- } \\
\text { NESS } \\
\text { (MG/L } \\
\text { AS } \\
\text { CACO3) }\end{array}$ & $\begin{array}{c}\text { CALCIUM, } \\
\text { DIS- } \\
\text { SOLVED } \\
\text { (MG/L } \\
\text { AS CA) }\end{array}$ & $\begin{array}{l}\text { MAGNE- } \\
\text { SIUM, } \\
\text { DIS- } \\
\text { SOLVED } \\
\text { (MG/L } \\
\text { AS MG) }\end{array}$ & $\begin{array}{l}\text { SODIUM, } \\
\text { DIS- } \\
\text { SOLVED } \\
\text { (MG/L } \\
\text { AS NA) }\end{array}$ & $\begin{array}{l}\text { POTAS- } \\
\text { SIUM, } \\
\text { DIS- } \\
\text { SOLVED } \\
\text { (MG/L } \\
\text { AS K) }\end{array}$ & $\begin{array}{l}\text { FLUO- } \\
\text { RIDE, } \\
\text { DIS- } \\
\text { SOLVED } \\
\text { (MG/L } \\
\text { AS F) }\end{array}$ \\
\hline $\begin{array}{l}85-08-07 \\
85-08-08 \\
84-05-31 \\
84-07-24 \\
84-10-12\end{array}$ & $\begin{array}{l}1.1 \\
.4 \\
3.3 \\
8.0 \\
5.7\end{array}$ & $\begin{array}{l}641 \\
641 \\
642 \\
646 \\
643\end{array}$ & $\begin{array}{l}150 \\
150 \\
210 \\
210 \\
180\end{array}$ & $\begin{array}{l}180 \\
180 \\
260 \\
260 \\
220\end{array}$ & $\begin{array}{l}0 \\
0 \\
0 \\
0 \\
0\end{array}$ & $\begin{array}{c}14 \\
5.7 \\
13 \\
21 \\
14\end{array}$ & $\begin{array}{r}160 \\
140 \\
-- \\
-- \\
--\end{array}$ & $\begin{array}{l}47 \\
39 \\
-- \\
-- \\
--\end{array}$ & $\begin{array}{r}11 \\
9.5 \\
-- \\
--\end{array}$ & $\begin{array}{l}12 \\
12 \\
-- \\
--\end{array}$ & $\begin{array}{r}3.7 \\
4.0 \\
2.5 \\
-- \\
2.6\end{array}$ & $\begin{array}{r}0.70 \\
.80 \\
-- \\
-- \\
--\end{array}$ \\
\hline $\begin{array}{l}84-04-12 \\
84-05-31 \\
84-07-24 \\
84-10-12 \\
84-05-31\end{array}$ & $\begin{array}{r}.9 \\
.4 \\
1.0 \\
.2 \\
4.8\end{array}$ & $\begin{array}{l}-\overline{642} \\
646 \\
644 \\
639\end{array}$ & $\begin{array}{l}180 \\
190 \\
200 \\
180 \\
200\end{array}$ & $\begin{array}{l}220 \\
230 \\
240 \\
220 \\
240\end{array}$ & $\begin{array}{l}0 \\
0 \\
0 \\
0 \\
0\end{array}$ & $\begin{array}{l}18 \\
15 \\
19 \\
18 \\
15\end{array}$ & $\begin{array}{l}-- \\
-- \\
-- \\
-- \\
--\end{array}$ & $\begin{array}{l}-- \\
-- \\
-- \\
-- \\
--\end{array}$ & $\begin{array}{l}-- \\
-- \\
-- \\
-- \\
--\end{array}$ & $\begin{array}{l}-- \\
-- \\
-- \\
--\end{array}$ & $\begin{array}{r}-- \\
3.0 \\
-- \\
2.9 \\
3.0\end{array}$ & $\begin{array}{l}-- \\
-- \\
-- \\
-- \\
--\end{array}$ \\
\hline $\begin{array}{l}84-07-25 \\
84-10-11 \\
84-03-02 \\
84-03-01 \\
84-04-11\end{array}$ & $\begin{array}{r}4.5 \\
3.3 \\
-- \\
-- \\
4.5\end{array}$ & $\begin{array}{r}647 \\
637 \\
-- \\
-- \\
643\end{array}$ & $\begin{array}{l}210 \\
230 \\
210 \\
220 \\
230\end{array}$ & $\begin{array}{l}260 \\
280 \\
260 \\
270 \\
280\end{array}$ & $\begin{array}{l}0 \\
0 \\
0 \\
0 \\
0\end{array}$ & $\begin{array}{l}21 \\
18 \\
16 \\
22 \\
22\end{array}$ & $\begin{array}{r}-- \\
230 \\
240 \\
--\end{array}$ & $\begin{array}{l}-- \\
-2 \\
67 \\
69 \\
--\end{array}$ & $\begin{array}{l}16^{--} \\
16 \\
\ldots\end{array}$ & $14^{--}$ & $\begin{array}{l}3 .- \\
3.6 \\
3.5 \\
3.2\end{array}$ & $\begin{array}{l}- \\
-- \\
.70 \\
.70 \\
--\end{array}$ \\
\hline $\begin{array}{l}84-05-24 \\
84-07-25 \\
84-10-12 \\
84-02-02 \\
84-03-06\end{array}$ & $\begin{array}{r}6.3 \\
5.2 \\
4.8 \\
.1 \\
.3\end{array}$ & $\begin{array}{r}640 \\
645 \\
643 \\
-2 \\
647\end{array}$ & $\begin{array}{l}180 \\
210 \\
220 \\
220 \\
210\end{array}$ & $\begin{array}{l}220 \\
250 \\
270 \\
270 \\
260\end{array}$ & $\begin{array}{l}0 \\
0 \\
0 \\
0 \\
0\end{array}$ & $\begin{array}{l}18 \\
16 \\
22 \\
34 \\
16\end{array}$ & $\overline{--}$ & $\begin{array}{l}-- \\
-- \\
-2 \\
65\end{array}$ & $17^{--}$ & $25^{--}$ & $\begin{array}{r}2.8 \\
-- \\
3.3 \\
3.6 \\
-2\end{array}$ & $\begin{array}{l}-- \\
-- \\
-- \\
80 \\
--\end{array}$ \\
\hline $\begin{array}{l}84-04-11 \\
84-05-22 \\
84-07-24 \\
84-10-10 \\
84-03-06\end{array}$ & $\begin{array}{l}.2 \\
.4 \\
.8 \\
.6 \\
.--\end{array}$ & $\begin{array}{l}643 \\
645 \\
648 \\
640 \\
647\end{array}$ & $\begin{array}{l}240 \\
190 \\
210 \\
210 \\
220\end{array}$ & $\begin{array}{l}300 \\
230 \\
260 \\
250 \\
270\end{array}$ & $\begin{array}{l}0 \\
0 \\
0 \\
0 \\
0\end{array}$ & $\begin{array}{l}19 \\
23 \\
26 \\
16 \\
17\end{array}$ & $2 \overline{-}$ & $\begin{array}{l}-- \\
-- \\
60 \\
--\end{array}$ & $14^{--}$ & $23^{--}$ & $\begin{array}{r}3.5 \\
3.4 \\
-- \\
2.9 \\
--\end{array}$ & $\begin{array}{c}-- \\
-- \\
-\overline{80} \\
--\end{array}$ \\
\hline $\begin{array}{l}84-05-31 \\
84-07-24 \\
84-10-12 \\
84-05-31 \\
84-07-25\end{array}$ & $\begin{array}{l}.3 \\
3.6 \\
1.8 \\
6.5 \\
5.3\end{array}$ & $\begin{array}{l}639 \\
646 \\
644 \\
641 \\
646\end{array}$ & $\begin{array}{l}220 \\
230 \\
210 \\
170 \\
190\end{array}$ & $\begin{array}{l}270 \\
280 \\
260 \\
210 \\
230\end{array}$ & $\begin{array}{l}0 \\
0 \\
0 \\
0 \\
0\end{array}$ & $\begin{array}{l}17 \\
22 \\
21 \\
8.4 \\
12\end{array}$ & $\begin{array}{l}-- \\
-- \\
-- \\
--\end{array}$ & $\begin{array}{l}-- \\
-- \\
-- \\
--\end{array}$ & $\begin{array}{l}-- \\
-- \\
--\end{array}$ & $\begin{array}{l}-- \\
-- \\
-- \\
-- \\
--\end{array}$ & $\begin{array}{r}3.3 \\
-.- \\
3.1 \\
3.1 \\
. .\end{array}$ & $\begin{array}{l}-- \\
-- \\
-- \\
--\end{array}$ \\
\hline $\begin{array}{l}84-10-11 \\
84-03-01 \\
84-04-12 \\
84-05-23 \\
84-07-25\end{array}$ & $\begin{array}{r}4.2 \\
-.2 \\
.7 \\
2.7 \\
2.8\end{array}$ & $\begin{array}{r}636 \\
-- \\
642 \\
641 \\
645\end{array}$ & $\begin{array}{l}200 \\
200 \\
230 \\
250 \\
250\end{array}$ & $\begin{array}{l}240 \\
240 \\
280 \\
310 \\
300\end{array}$ & $\begin{array}{l}0 \\
0 \\
0 \\
0 \\
0\end{array}$ & $\begin{array}{l}15 \\
15 \\
28 \\
39 \\
30\end{array}$ & $\begin{array}{r}-\overline{-} \\
200 \\
-- \\
--\end{array}$ & $\begin{array}{l}-\overline{63} \\
-- \\
--\end{array}$ & $11^{--}$ & $10^{--}$ & $\begin{array}{l}3.0 \\
2.3 \\
2.4 \\
2.7 \\
-.\end{array}$ & $\begin{array}{l}-\overline{-} \\
.80 \\
-- \\
--\end{array}$ \\
\hline $\begin{array}{l}84-10-11 \\
84-02-28 \\
84-04-10 \\
84-05-22 \\
84-07-25\end{array}$ & $\begin{array}{r}2.2 \\
2.7 \\
4.5 \\
.4 \\
.3\end{array}$ & $\begin{array}{r}636 \\
-2 \\
636 \\
646 \\
646\end{array}$ & $\begin{array}{l}220 \\
150 \\
160 \\
190 \\
200\end{array}$ & $\begin{array}{l}270 \\
190 \\
190 \\
230 \\
240\end{array}$ & $\begin{array}{l}0 \\
0 \\
0 \\
0 \\
0\end{array}$ & $\begin{array}{l}27 \\
9.5 \\
9.5 \\
18 \\
15\end{array}$ & $\begin{array}{r}-- \\
170 \\
170 \\
-- \\
--\end{array}$ & $\begin{array}{l}-- \\
51 \\
50 \\
-- \\
--\end{array}$ & $\begin{array}{l}11^{--} \\
11 \\
--\end{array}$ & $\begin{array}{l}11^{--} \\
11 \\
--\end{array}$ & $\begin{array}{l}2.4 \\
2.1 \\
2.3 \\
2.3 \\
--\end{array}$ & $\begin{array}{l}-- \\
.70 \\
.70 \\
-- \\
--\end{array}$ \\
\hline
\end{tabular}


Table 3.--Ground-water quality data, 1984 and 1985--Continued

\begin{tabular}{|c|c|c|c|c|c|c|c|c|c|c|c|c|}
\hline $\begin{array}{c}\text { DATE } \\
\text { OF } \\
\text { SAMPLE }\end{array}$ & $\begin{array}{l}\text { CHLO- } \\
\text { RIDE, } \\
\text { DIS- } \\
\text { SOLVED } \\
\text { (MG/L } \\
\text { AS CL) }\end{array}$ & $\begin{array}{l}\text { SULFATE, } \\
\text { DIS- } \\
\text { SOLVED } \\
\text { (MG/L } \\
\text { AS SO4) }\end{array}$ & $\begin{array}{l}\text { SILICA, } \\
\text { DIS- } \\
\text { SOLVED } \\
\text { (MG/L } \\
\text { AS } \\
\text { SIO2) }\end{array}$ & $\begin{array}{l}\text { CARBON, } \\
\text { ORGANIC, } \\
\text { DISSOLVED } \\
\text { (MG/L } \\
\text { AS C) }\end{array}$ & $\begin{array}{l}\text { METHY- } \\
\text { LENE } \\
\text { BLUE } \\
\text { ACTIVE } \\
\text { SUB- } \\
\text { STANCE } \\
(\text { MG/L) }\end{array}$ & $\begin{array}{l}\text { NITRO- } \\
\text { GEN, } \\
\text { NO2+NO3 } \\
\text { DIS- } \\
\text { SOLVED } \\
\text { (MG/L } \\
\text { AS N) }\end{array}$ & $\begin{array}{l}\text { NITRO- } \\
\text { GEN, } \\
\text { AMMONIA } \\
\text { DIS- } \\
\text { SOLVED } \\
\text { (MG/L } \\
\text { AS N) }\end{array}$ & $\begin{array}{l}\text { NITRO- } \\
\text { GEN, AM- } \\
\text { MONIA + } \\
\text { ORGANIC } \\
\text { DISSOLVED } \\
\text { (MG/L } \\
\text { AS N) }\end{array}$ & $\begin{array}{l}\text { NITRO- } \\
\text { GEN, } \\
\text { ORGANIC } \\
\text { DIS- } \\
\text { SOLVED } \\
\text { (MG/L } \\
\text { AS N) }\end{array}$ & $\begin{array}{c}\text { NITRO- } \\
\text { GEN, } \\
\text { AMMONIA, } \\
\text { DIS- } \\
\text { SOLVED } \\
\text { (MG/L } \\
\text { AS NH4) }\end{array}$ & $\begin{array}{l}\text { PHOS- } \\
\text { PHORUS, } \\
\text { DIS- } \\
\text { SOLVED } \\
\text { (MG/L } \\
\text { AS P) }\end{array}$ & $\begin{array}{l}\text { IRON, } \\
\text { DIS- } \\
\text { SOLVED } \\
\text { (UG/L } \\
\text { AS FE) }\end{array}$ \\
\hline $\begin{array}{l}85-08-07 \\
85-08-08 \\
84-05-31 \\
84-07-24 \\
84-10-12\end{array}$ & $\begin{array}{r}8.5 \\
7.3 \\
-- \\
-- \\
--\end{array}$ & $\begin{array}{l}26 \\
24 \\
-- \\
-- \\
--\end{array}$ & $\begin{array}{l}18 \\
20 \\
-- \\
-- \\
--\end{array}$ & $\begin{array}{l}1.3 \\
1.9 \\
.80 \\
-- \\
--\end{array}$ & $\begin{array}{l}-- \\
-- \\
-- \\
-- \\
--\end{array}$ & $\begin{array}{c}<0.10 \\
<.10 \\
.35 \\
.67 \\
1.5\end{array}$ & $\begin{array}{r}0.140 \\
.470 \\
<.010 \\
<.010 \\
<.010\end{array}$ & $\begin{array}{l}0.50 \\
1.0 \\
.30 \\
.40 \\
.20\end{array}$ & $\begin{array}{r}0.36 \\
.53 \\
-- \\
-- \\
--\end{array}$ & $\begin{array}{r}0.18 \\
.61 \\
-- \\
-- \\
--\end{array}$ & $\begin{array}{r}-- \\
0.010 \\
-- \\
--\end{array}$ & $\begin{array}{r}46 \\
1100 \\
10 \\
-- \\
--\end{array}$ \\
\hline $\begin{array}{l}84-04-12 \\
84-05-31 \\
84-07-24 \\
84-10-12 \\
84-05-31\end{array}$ & $\begin{array}{l}-- \\
-- \\
-- \\
--\end{array}$ & $\begin{array}{l}-- \\
-- \\
-- \\
-- \\
--\end{array}$ & $\begin{array}{l}-- \\
-- \\
-- \\
-- \\
--\end{array}$ & $\begin{array}{r}-- \\
.70 \\
-- \\
-- \\
.90\end{array}$ & $\begin{array}{l}-- \\
-- \\
-- \\
-- \\
--\end{array}$ & $\begin{array}{l}1.8 \\
.85 \\
.84 \\
2.4\end{array}$ & $\begin{array}{r}<.-- \\
<.010 \\
<.010 \\
<.010 \\
.100\end{array}$ & $\begin{array}{l}1.0 \\
.60 \\
<.20 \\
.70\end{array}$ & $\begin{array}{l}-- \\
-- \\
-- \\
-- \\
60\end{array}$ & $\begin{array}{l}-- \\
-- \\
-- \\
-- \\
.13\end{array}$ & $\begin{array}{r}.010 \\
-0 \\
.070\end{array}$ & $\begin{array}{l}--\overline{10} \\
-- \\
\overline{20}\end{array}$ \\
\hline $\begin{array}{l}84-07-25 \\
84-10-11 \\
84-03-02 \\
84-03-01 \\
84-04-11\end{array}$ & $\begin{array}{l}13^{--} \\
11 \\
--\end{array}$ & $\begin{array}{l}-- \\
-- \\
35 \\
35 \\
34\end{array}$ & $\begin{array}{l}-- \\
-- \\
23 \\
22 \\
--\end{array}$ & $\begin{array}{l}-- \\
-- \\
.50 \\
1.1 \\
.60\end{array}$ & $\begin{array}{c}-- \\
-- \\
-- \\
0.02\end{array}$ & $\begin{array}{l}2.7 \\
3.4 \\
2.5 \\
2.2 \\
2.1\end{array}$ & $\begin{array}{l}.010 \\
.080 \\
.040 \\
.080 \\
.040\end{array}$ & $\begin{array}{l}.40 \\
.50 \\
-- \\
-- \\
1.1\end{array}$ & $\begin{array}{r}.39 \\
.42 \\
-- \\
-1.1\end{array}$ & $\begin{array}{l}.01 \\
.10 \\
.05 \\
.10 \\
.05\end{array}$ & $\begin{array}{l}-- \\
-- \\
-- \\
-- \\
--\end{array}$ & $\begin{array}{r}-- \\
-- \\
4 \\
12 \\
9\end{array}$ \\
\hline $\begin{array}{l}84-05-24 \\
84-07-25 \\
84-10-12 \\
84-02-02 \\
84-03-06\end{array}$ & $27_{--}^{--}$ & $\begin{array}{l}-- \\
-- \\
-- \\
--\end{array}$ & $\begin{array}{l}-- \\
-- \\
-- \\
--\end{array}$ & $\begin{array}{c}.70 \\
-- \\
-- \\
-- \\
.80\end{array}$ & $\begin{array}{l}-- \\
-- \\
-- \\
-- \\
--\end{array}$ & $\begin{array}{l}1.2 \\
1.9 \\
2.2 \\
<.10 \\
<.10\end{array}$ & $\begin{array}{r}<.010 \\
.010 \\
<.010 \\
.210\end{array}$ & $\begin{array}{r}.50 \\
.20 \\
.50 \\
-- \\
--\end{array}$ & $\begin{array}{l}-- \\
19 \\
-- \\
-- \\
--\end{array}$ & $\begin{array}{c}-\overline{01} \\
-- \\
-- \\
.27\end{array}$ & $\begin{array}{c}.030 \\
-- \\
-- \\
-- \\
--\end{array}$ & $\begin{array}{r}10 \\
-- \\
-- \\
59 \\
100\end{array}$ \\
\hline $\begin{array}{l}84-04-11 \\
84-05-22 \\
84-07-24 \\
84-10-10 \\
84-03-06\end{array}$ & $20^{--}$ & $\begin{array}{l}33 \\
-- \\
-- \\
27 \\
--\end{array}$ & $\begin{array}{l}-- \\
-- \\
-- \\
--\end{array}$ & $\begin{array}{l}1.0 \\
.70 \\
-- \\
2.7 \\
1.1\end{array}$ & $\begin{array}{c}<.01 \\
-- \\
-- \\
-- \\
--\end{array}$ & $\begin{array}{l}.36 \\
5.2 \\
1.5 \\
1.1 \\
<.10\end{array}$ & $\begin{array}{l}.150 \\
.370 \\
.030 \\
.030 \\
.150\end{array}$ & $\begin{array}{r}.30 \\
1.2 \\
.40 \\
.40 \\
--\end{array}$ & $\begin{array}{l}.15 \\
.83 \\
.37 \\
.37 \\
--\end{array}$ & $\begin{array}{l}.19 \\
.48 \\
.04 \\
.04 \\
.19\end{array}$ & $\begin{array}{r}.020 \\
.010 \\
--\end{array}$ & $\begin{array}{r}23 \\
29 \\
8 \\
4 \\
--\end{array}$ \\
\hline $\begin{array}{l}84-05-31 \\
84-07-24 \\
84-10-12 \\
84-05-31 \\
84-07-25\end{array}$ & $\begin{array}{l}-- \\
-- \\
-- \\
--\end{array}$ & $\begin{array}{l}-- \\
-- \\
-- \\
-- \\
--\end{array}$ & $\begin{array}{l}-- \\
-- \\
-- \\
-- \\
--\end{array}$ & $\begin{array}{c}.70 \\
-- \\
-- \\
.70 \\
--\end{array}$ & $\begin{array}{l}-- \\
-- \\
-- \\
-- \\
--\end{array}$ & $\begin{array}{r}.42 \\
1.5 \\
.79 \\
.99 \\
.99\end{array}$ & $\begin{array}{r}.020 \\
.010 \\
<.010 \\
<.010 \\
<.010\end{array}$ & $\begin{array}{r}.50 \\
.30 \\
<.20 \\
.30 \\
.80\end{array}$ & $\begin{array}{r}.48 \\
.29 \\
-- \\
-- \\
--\end{array}$ & $\begin{array}{r}.03 \\
.01 \\
-- \\
-- \\
--\end{array}$ & $\begin{array}{r}.010 \\
-- \\
-0 \\
.010 \\
--\end{array}$ & $\begin{array}{r}7 \\
-- \\
-\overline{10} \\
--\end{array}$ \\
\hline $\begin{array}{l}84-10-11 \\
84-03-01 \\
84-04-12 \\
84-05-23 \\
84-07-25\end{array}$ & $\begin{array}{l}--\overline{9} \\
-- \\
-- \\
--\end{array}$ & $\begin{array}{l}-- \\
30 \\
33 \\
-- \\
--\end{array}$ & $\begin{array}{l}-- \\
21 \\
-- \\
-- \\
--\end{array}$ & $\begin{array}{l}.-- \\
.60 \\
.60 \\
.50 \\
--\end{array}$ & $\begin{array}{l}-- \\
-\overline{01} \\
-- \\
--\end{array}$ & $\begin{array}{l}1.6 \\
<.10 \\
.35 \\
.36 \\
1.2\end{array}$ & $\begin{array}{r}.020 \\
.040 \\
.030 \\
<.010 \\
<.010\end{array}$ & $\begin{array}{l}.30 \\
-0 \\
5.0 \\
.40 \\
.40\end{array}$ & $\begin{array}{c}.28 \\
-- \\
5.0 \\
--\end{array}$ & $\begin{array}{l}.03 \\
.05 \\
.04 \\
-- \\
--\end{array}$ & $\begin{array}{r}.010 \\
-- \\
-\overline{-} \\
.050 \\
--\end{array}$ & $\begin{array}{r}50 \\
4 \\
5 \\
<3 \\
--\end{array}$ \\
\hline $\begin{array}{l}84-10-11 \\
84-02-28 \\
84-04-10 \\
84-05-22 \\
84-07-25\end{array}$ & $\begin{array}{c}9.7 \\
11 \\
--\end{array}$ & $\begin{array}{l}--- \\
32 \\
32 \\
-- \\
--\end{array}$ & $\begin{array}{l}-- \\
17 \\
16 \\
-- \\
--\end{array}$ & $\begin{array}{l}.- \\
.60 \\
.50 \\
.60 \\
.-\end{array}$ & $\begin{array}{c}-- \\
-01 \\
--\end{array}$ & $\begin{array}{r}.75 \\
.25 \\
.48 \\
.26 \\
<.10\end{array}$ & $\begin{array}{r}<.010 \\
.040 \\
.040 \\
<.010 \\
.020\end{array}$ & $\begin{array}{r}.20 \\
-20 \\
.20 \\
.70 \\
.30\end{array}$ & $\begin{array}{l}-- \\
.16 \\
-\overline{-} \\
.28\end{array}$ & $\begin{array}{r}.-5 \\
.05 \\
.05 \\
-.- \\
.03\end{array}$ & $\begin{array}{r}.010 \\
-- \\
.050 \\
--\end{array}$ & $\begin{array}{r}12 \\
4 \\
<3 \\
14 \\
--\end{array}$ \\
\hline
\end{tabular}


Table 3.--Ground-water quality data 1984 and $1985--C o n t i n u e d$

\begin{tabular}{|c|c|c|c|c|c|c|c|c|c|c|c|}
\hline $\begin{array}{l}\text { WELL } \\
\text { NUM- } \\
\text { BER }\end{array}$ & $\begin{array}{l}\text { WELL } \\
\text { LOCA- } \\
\text { TION }\end{array}$ & $\begin{array}{c}\text { DATE } \\
\text { OF } \\
\text { SAMPLE }\end{array}$ & TIME & $\begin{array}{l}\text { SPE- } \\
\text { CIFIC } \\
\text { CONDUC- } \\
\text { TANCE* } \\
\text { (UMHOS) }\end{array}$ & $\begin{array}{l}\text { SPE- } \\
\text { CIFIC } \\
\text { CONDUC- } \\
\text { TANCE, } \\
\text { LAB } \\
\text { (UMHOS) }\end{array}$ & $\begin{array}{l}\text { SOLIDS, } \\
\text { SUM OF } \\
\text { CONSTI- } \\
\text { TUENTS, } \\
\text { DIS- } \\
\text { SOLVED } \\
\text { (MG/L) }\end{array}$ & $\begin{array}{l}\text { SOLIDS, } \\
\text { RESIDUE } \\
\text { AT } 180 \\
\text { DEG. C, } \\
\text { DIS- } \\
\text { SOLVED } \\
\text { (MG/L) }\end{array}$ & $\begin{array}{l}\text { SOLIDS/ } \\
\text { SPE- } \\
\text { CIFIC } \\
\text { CONDUC- } \\
\text { TANCE, } \\
\text { RATIO }\end{array}$ & $\begin{array}{l}\text { CATION/ } \\
\text { ANION, } \\
\text { PERCENT } \\
\text { DIFFER- } \\
\text { ENCE }\end{array}$ & $\begin{array}{l}\text { TEMPER- } \\
\text { ATURE* } \\
\text { (DEG. C) }\end{array}$ & $\begin{array}{c}\text { PH* } \\
\text { (STAN- } \\
\text { DARD } \\
\text { UNITS) }\end{array}$ \\
\hline $\begin{array}{l}82 \\
85\end{array}$ & $\begin{array}{c}\text { (CONTINUED) } \\
2 S-35 E-32 D A C A 1\end{array}$ & $\begin{array}{l}84-10-11 \\
84-01-31 \\
84-03-06 \\
84-05-24 \\
84-07-26\end{array}$ & $\begin{array}{l}1300 \\
1430 \\
1025 \\
1430 \\
1130\end{array}$ & $\begin{array}{l}399 \\
510 \\
495 \\
353 \\
438\end{array}$ & $\begin{array}{l}412 \\
501 \\
506 \\
356 \\
357\end{array}$ & $\begin{array}{c}240 \\
310 \\
-- \\
-- \\
--\end{array}$ & $\begin{array}{r}220 \\
284 \\
298 \\
-- \\
--\end{array}$ & $\begin{array}{r}0.55 \\
.57 \\
.60 \\
-- \\
--\end{array}$ & $\begin{array}{r}-1.15 \\
.21 \\
-- \\
-- \\
--\end{array}$ & $\begin{array}{r}14.5 \\
11.0 \\
11.5 \\
9.5 \\
10.0\end{array}$ & $\begin{array}{l}7.3 \\
7.4 \\
7.2 \\
7.3 \\
7.4\end{array}$ \\
\hline 86. & $2 S-35 E-32 D A D A 1$ & $\begin{array}{l}84-10-04 \\
84-02-02 \\
84-03-06 \\
84-04-11 \\
84-05-23\end{array}$ & $\begin{array}{l}1300 \\
1530 \\
1600 \\
1700 \\
1810\end{array}$ & $\begin{array}{l}479 \\
497 \\
488 \\
461 \\
450\end{array}$ & $\begin{array}{l}489 \\
511 \\
508 \\
514 \\
444\end{array}$ & $\begin{array}{l}310 \\
-- \\
--\end{array}$ & $\begin{array}{l}303 \\
302 \\
288 \\
--\end{array}$ & $\begin{array}{l}.0 \\
.61 \\
.62 \\
.62 \\
.-\end{array}$ & $\begin{array}{r}-- \\
-2.19 \\
-- \\
--\end{array}$ & $\begin{array}{l}12.0 \\
12.0 \\
12.5 \\
12.5 \\
12.5\end{array}$ & $\begin{array}{l}7.3 \\
7.3 \\
7.3 \\
7.2 \\
7.3\end{array}$ \\
\hline 87. & $2 S-35 E-32 D A D B 1$ & $\begin{array}{l}84-07-24 \\
84-10-10 \\
84-02-02 \\
84-03-06 \\
84-04-11\end{array}$ & $\begin{array}{l}1945 \\
1845 \\
1620 \\
1200 \\
1615\end{array}$ & $\begin{array}{l}475 \\
529 \\
509 \\
512 \\
515\end{array}$ & $\begin{array}{l}481 \\
533 \\
515 \\
515 \\
--\end{array}$ & $\frac{-}{--}$ & $\begin{array}{l}-- \\
300 \\
306 \\
--\end{array}$ & $\begin{array}{l}.- \\
.2 \\
.59 \\
.60 \\
--\end{array}$ & $\begin{array}{r}-- \\
-1.85 \\
--\end{array}$ & $\begin{array}{l}12.0 \\
12.0 \\
12.5 \\
12.0 \\
12.5\end{array}$ & $\begin{array}{l}7.3 \\
7.0 \\
7.4 \\
7.3 \\
7.3\end{array}$ \\
\hline 88 & $2 S-35 E-32 D B C A 1$ & $\begin{array}{l}84-05-24 \\
84-02-02 \\
84-03-07 \\
84-04-13 \\
84-05-31\end{array}$ & $\begin{array}{l}1815 \\
1345 \\
1415 \\
1215 \\
1115\end{array}$ & $\begin{array}{l}402 \\
477 \\
468 \\
439 \\
367\end{array}$ & $\begin{array}{l}405 \\
469 \\
478 \\
447 \\
375\end{array}$ & 280 & $\begin{array}{l}-- \\
268 \\
281 \\
259 \\
--\end{array}$ & $\begin{array}{l}.- \\
.56 \\
.60 \\
.59 \\
.-\end{array}$ & $\begin{array}{r}-\overline{-} \\
-1 . \\
-- \\
--\end{array}$ & $\begin{array}{l}11.5 \\
12.0 \\
12.0 \\
11.0 \\
10.0\end{array}$ & $\begin{array}{l}7.3 \\
7.3 \\
7.4 \\
7.5 \\
7.6\end{array}$ \\
\hline 89 & $2 S-35 E-32 D C C A I$ & $\begin{array}{l}84-07-26 \\
84-10-12 \\
84-03-07 \\
84-04-10 \\
84-05-31\end{array}$ & $\begin{array}{l}1230 \\
1315 \\
1715 \\
1100 \\
1315\end{array}$ & $\begin{array}{l}381 \\
443 \\
442 \\
413 \\
364\end{array}$ & $\begin{array}{l}385 \\
455 \\
452 \\
420 \\
364\end{array}$ & $\frac{--}{--}$ & $\begin{array}{r}-- \\
-- \\
263 \\
272 \\
--\end{array}$ & $\begin{array}{l}-- \\
-- \\
.62 \\
.66 \\
--\end{array}$ & $\begin{array}{r}-- \\
-1.35 \\
--\end{array}$ & $\begin{array}{r}9.5 \\
11.0 \\
11.0 \\
10.5 \\
10.0\end{array}$ & $\begin{array}{l}7.5 \\
7.3 \\
7.5 \\
7.4 \\
7.6\end{array}$ \\
\hline $\begin{array}{l}92 . \\
93 .\end{array}$ & $\begin{array}{l}2 S-35 E-32 D C C C 1 \\
2 S-35 E-32 D D B D I\end{array}$ & $\begin{array}{l}84-07-19 \\
84-04-13 \\
84-04-12 \\
84-05-24 \\
84-07-25\end{array}$ & $\begin{array}{l}1215 \\
1850 \\
1545 \\
1000 \\
1510\end{array}$ & $\begin{array}{l}382 \\
389 \\
396 \\
371 \\
324\end{array}$ & $\begin{array}{l}382 \\
387 \\
407 \\
376 \\
344\end{array}$ & $\begin{array}{l}-- \\
-- \\
-- \\
--\end{array}$ & $\begin{array}{r}-- \\
215 \\
235 \\
-- \\
--\end{array}$ & $\begin{array}{r}-- \\
.55 \\
.59 \\
-- \\
--\end{array}$ & $\begin{array}{l}- \\
-- \\
-- \\
--\end{array}$ & $\begin{array}{r}11.0 \\
9.0 \\
9.0 \\
10.0 \\
14.0\end{array}$ & $\begin{array}{l}7.4 \\
7.7 \\
7.5 \\
7.6 \\
7.6\end{array}$ \\
\hline \multirow[t]{2}{*}{95.} & $2 S-35 E-33 A B A D 1$ & $\begin{array}{l}84-10-11 \\
84-02-02 \\
84-02-28 \\
84-04-13 \\
84-05-25\end{array}$ & $\begin{array}{l}1615 \\
1000 \\
1033 \\
1615 \\
0950\end{array}$ & $\begin{array}{l}347 \\
545 \\
519 \\
696 \\
647\end{array}$ & $\begin{array}{l}354 \\
516 \\
522 \\
670 \\
\ldots\end{array}$ & $\begin{array}{l}330 \\
320 \\
-- \\
--\end{array}$ & $\begin{array}{r}-- \\
303 \\
305 \\
-- \\
--\end{array}$ & $\begin{array}{r}.- \\
.56 \\
.59 \\
-- \\
--\end{array}$ & $\begin{array}{r}-5 \\
-5.90 \\
-2.66 \\
\ldots\end{array}$ & $\begin{array}{l}15.5 \\
11.5 \\
11.5 \\
12.0 \\
11.5\end{array}$ & $\begin{array}{l}7.6 \\
7.5 \\
7.6 \\
7.3 \\
7.4\end{array}$ \\
\hline & & $\begin{array}{l}84-07-27 \\
84-10-04 \\
85-04-05 \\
85-04-17 \\
85-05-03\end{array}$ & $\begin{array}{l}0915 \\
1500 \\
1000 \\
1400 \\
1200\end{array}$ & $\begin{array}{r}1070 \\
694 \\
1450 \\
1300 \\
1220\end{array}$ & $\begin{array}{r}1060 \\
701 \\
-- \\
--\end{array}$ & $\begin{array}{l}-- \\
430 \\
-- \\
--\end{array}$ & $\begin{array}{l}-- \\
-- \\
-- \\
--\end{array}$ & $\begin{array}{l}.- \\
.62 \\
-- \\
--\end{array}$ & $\begin{array}{r}-3 . \\
-38 \\
-- \\
--\end{array}$ & $\begin{array}{r}10.5 \\
11.0 \\
-0 \\
10.0 \\
--\end{array}$ & $\begin{array}{r}6.8 \\
7.0 \\
-. \\
7.4 \\
--.\end{array}$ \\
\hline
\end{tabular}


Table 3.--Ground-water quality data, 1984 and 1985--Continued

\begin{tabular}{|c|c|c|c|c|}
\hline $\begin{array}{c}\text { DATE } \\
\text { OF } \\
\text { SAMPLE }\end{array}$ & $\begin{array}{l}\text { IRON, } \\
\text { SUS- } \\
\text { PENDED } \\
\text { RECOV- } \\
\text { ERABLE } \\
\text { (UG/L } \\
\text { AS FE) }\end{array}$ & $\begin{array}{l}\text { IRON, } \\
\text { TOTAL } \\
\text { RECOV- } \\
\text { ERABLE } \\
\text { (UG/L } \\
\text { AS FE) }\end{array}$ & $\begin{array}{l}\text { MANGA- } \\
\text { NESE, } \\
\text { DIS- } \\
\text { SOLVED } \\
\text { (UG/L } \\
\text { AS MN) }\end{array}$ & $\begin{array}{l}\text { 2INC, } \\
\text { DIS- } \\
\text { SOLVED } \\
\text { (UG/L } \\
\text { AS } 2 \mathrm{~N} \text { ) }\end{array}$ \\
\hline $\begin{array}{l}85-08-07 \\
85-08-08 \\
84-05-31 \\
84-07-24 \\
84-10-12\end{array}$ & $\begin{array}{l}-- \\
-- \\
--\end{array}$ & $\begin{array}{l}-- \\
120 \\
--\end{array}$ & $\begin{array}{r}140 \\
1500 \\
<10 \\
-- \\
--\end{array}$ & $\begin{array}{l}33 \\
17 \\
70 \\
70 \\
40\end{array}$ \\
\hline $\begin{array}{l}84-04-12 \\
84-05-31 \\
84-07-24 \\
84-10-12 \\
84-05-31\end{array}$ & $\begin{array}{l}-- \\
-- \\
--\end{array}$ & $\begin{array}{l}-\overline{20} \\
\overline{30}\end{array}$ & $\begin{array}{l}\overline{30} \\
\overline{-} \\
<10\end{array}$ & $\begin{array}{l}-\overline{80} \\
\overline{10} \\
40\end{array}$ \\
\hline $\begin{array}{l}84-07-25 \\
84-10-11 \\
84-03-02 \\
84-03-01 \\
84-04-11\end{array}$ & $\begin{array}{l}-- \\
=- \\
30\end{array}$ & $\begin{array}{l}-- \\
-- \\
40\end{array}$ & $\begin{array}{l}-- \\
-- \\
<1 \\
4\end{array}$ & $\begin{array}{l}\overline{40} \\
\overline{-} \\
180\end{array}$ \\
\hline $\begin{array}{l}84-05-24 \\
84-07-25 \\
84-10-12 \\
84-02-02 \\
84-03-06\end{array}$ & $\begin{array}{l}-- \\
-- \\
-- \\
--\end{array}$ & $\begin{array}{l}<10 \\
=- \\
-- \\
--\end{array}$ & $\begin{array}{l}<10 \\
\overline{--} \\
490 \\
600\end{array}$ & $\begin{array}{l}70 \\
-- \\
50 \\
--\end{array}$ \\
\hline $\begin{array}{l}84-04-11 \\
84-05-22 \\
84-07-24 \\
84-10-10 \\
84-03-06\end{array}$ & $\begin{array}{l}60 \\
-- \\
-- \\
--\end{array}$ & $\begin{array}{l}80 \\
20 \\
-- \\
-- \\
--\end{array}$ & $\begin{array}{r}580 \\
180 \\
23 \\
35 \\
--\end{array}$ & $\begin{array}{l}65 \\
58 \\
63 \\
21 \\
-\end{array}$ \\
\hline $\begin{array}{l}84-05-31 \\
84-07-24 \\
84-10-12 \\
84-05-31 \\
84-07-25\end{array}$ & $\begin{array}{l}-- \\
-- \\
--\end{array}$ & $\begin{array}{l}50 \\
-- \\
8 \\
--\end{array}$ & $\begin{array}{l}10 \\
\overline{20} \\
--\end{array}$ & $\begin{array}{r}110 \\
40 \\
90 \\
--\end{array}$ \\
\hline $\begin{array}{l}84-10-11 \\
84-03-01 \\
84-04-12 \\
84-05-23 \\
84-07-25\end{array}$ & $\begin{array}{l}-- \\
20 \\
--\end{array}$ & $\begin{array}{l}-- \\
20 \\
30 \\
--\end{array}$ & $\begin{array}{r}<10 \\
18 \\
7 \\
<1 \\
--\end{array}$ & $\begin{array}{l}80 \\
-- \\
76 \\
78 \\
--\end{array}$ \\
\hline $\begin{array}{l}84-10-11 \\
84-02-28 \\
84-04-10 \\
84-05-22 \\
84-07-25\end{array}$ & $\begin{array}{l}-- \\
-- \\
-- \\
--\end{array}$ & $\begin{array}{r}-\overline{-} \\
<10 \\
60 \\
--\end{array}$ & $\begin{array}{r}<1 \\
37 \\
6 \\
48 \\
--\end{array}$ & $\begin{array}{l}28 \\
-72 \\
79 \\
--\end{array}$ \\
\hline
\end{tabular}


Table 3.--Ground-water quality data, 1984 and 1985--Continued

\begin{tabular}{|c|c|c|c|c|c|c|c|c|c|c|c|c|}
\hline $\begin{array}{c}\text { DATE } \\
\text { OF } \\
\text { SAMPLE }\end{array}$ & $\begin{array}{c}\text { OXYGEN*, } \\
\text { DIS- } \\
\text { SOLVED } \\
\text { (MG/L) }\end{array}$ & $\begin{array}{l}\text { BARO- } \\
\text { METRIC } \\
\text { PRES- } \\
\text { SURE^ } \\
\text { (MM } \\
\text { OF HG) }\end{array}$ & $\begin{array}{c}\text { ALKA- } \\
\text { LINITY* } \\
\text { (MG/L } \\
\text { AS } \\
\text { CACO3) }\end{array}$ & $\begin{array}{l}\text { BICAR- } \\
\text { BONATE } \\
\text { FET-FLD } \\
\text { (MG/L AS } \\
\text { HCO3) }\end{array}$ & $\begin{array}{c}\text { CAR- } \\
\text { BONATE* } \\
\text { FET-FLD } \\
\text { (MG/L } \\
\text { AS } C O 3)\end{array}$ & $\begin{array}{l}\text { CARBON } \\
\text { DIOXIDE, } \\
\text { DIS- } \\
\text { SOLVED } \\
\text { (MG/L } \\
\text { AS CO2) }\end{array}$ & $\begin{array}{l}\text { HARD- } \\
\text { NESS } \\
\text { (MG/L } \\
\text { AS } \\
\text { CACO3) }\end{array}$ & $\begin{array}{l}\text { CALC IUM, } \\
\text { DIS- } \\
\text { SOLVED } \\
\text { (MG/L } \\
\text { AS CA) }\end{array}$ & $\begin{array}{l}\text { MAGNE- } \\
\text { SIUM, } \\
\text { DIS- } \\
\text { SOLVED } \\
\text { (MG/L } \\
\text { AS MG) }\end{array}$ & $\begin{array}{l}\text { SODIUM, } \\
\text { DIS- } \\
\text { SOLVED } \\
\text { (MG/L } \\
\text { AS NA) }\end{array}$ & $\begin{array}{l}\text { POTAS- } \\
\text { SIUM, } \\
\text { DIS- } \\
\text { SOLVED } \\
\text { (MG/L } \\
\text { AS K) }\end{array}$ & $\begin{array}{l}\text { FLUO- } \\
\text { RIDE, } \\
\text { DIS- } \\
\text { SOLVED } \\
\text { (MG/L } \\
\text { AS F) }\end{array}$ \\
\hline $\begin{array}{l}84-10-11 \\
84-01-31 \\
84-03-06 \\
84-05-24 \\
84-07-26\end{array}$ & $\begin{array}{l}0.2 \\
<.1 \\
4.7 \\
7.3 \\
4.7\end{array}$ & $\begin{array}{r}635 \\
-- \\
648 \\
--- \\
645\end{array}$ & $\begin{array}{l}180 \\
210 \\
200 \\
150 \\
200\end{array}$ & $\begin{array}{l}220 \\
260 \\
250 \\
180 \\
240\end{array}$ & $\begin{array}{l}0 \\
0 \\
0 \\
0 \\
0\end{array}$ & $\begin{array}{l}18 \\
16 \\
25 \\
14 \\
15\end{array}$ & $\begin{array}{r}190 \\
240 \\
-- \\
-- \\
--\end{array}$ & $\begin{array}{l}58 \\
70 \\
-- \\
-- \\
--\end{array}$ & $\begin{array}{l}11 \\
17 \\
=- \\
=-\end{array}$ & $\begin{array}{r}9.9 \\
14 \\
=- \\
=-\end{array}$ & $\begin{array}{r}2.3 \\
3.4 \\
2 .- \\
--\end{array}$ & $\begin{array}{r}0.70 \\
.60 \\
-- \\
--\end{array}$ \\
\hline $\begin{array}{l}84-10-04 \\
84-02-02 \\
84-03-06 \\
84-04-11 \\
84-05-23\end{array}$ & $\begin{array}{l}4.9 \\
-.- \\
4.6 \\
5.0 \\
5.4\end{array}$ & $\begin{array}{l}640 \\
-- \\
647 \\
643 \\
642\end{array}$ & $\begin{array}{l}210 \\
220 \\
220 \\
210 \\
190\end{array}$ & $\begin{array}{l}260 \\
270 \\
270 \\
260 \\
230\end{array}$ & $\begin{array}{l}0 \\
0 \\
0 \\
0 \\
0\end{array}$ & $\begin{array}{l}21 \\
22 \\
22 \\
26 \\
18\end{array}$ & $\begin{array}{r}240 \\
-- \\
--\end{array}$ & $\begin{array}{l}-- \\
69 \\
-- \\
--\end{array}$ & $16^{--}$ & $14^{--}$ & $\begin{array}{l}3.3 \\
3.4 \\
-- \\
3.3 \\
2.9\end{array}$ & $\begin{array}{l}.70 \\
-- \\
--\end{array}$ \\
\hline $\begin{array}{l}84-07-24 \\
84-10-10 \\
84-02-02 \\
84-03-06 \\
84-04-11\end{array}$ & $\begin{array}{r}5.4 \\
4.2 \\
-- \\
4.5 \\
5.6\end{array}$ & $\begin{array}{r}646 \\
637 \\
-- \\
648 \\
--\end{array}$ & $\begin{array}{l}210 \\
230 \\
230 \\
220 \\
220\end{array}$ & $\begin{array}{l}260 \\
280 \\
280 \\
270 \\
270\end{array}$ & $\begin{array}{l}0 \\
0 \\
0 \\
0 \\
0\end{array}$ & $\begin{array}{l}21 \\
44 \\
18 \\
22 \\
22\end{array}$ & $\begin{array}{r}-- \\
240 \\
--\end{array}$ & $\begin{array}{l}-- \\
70 \\
-- \\
--\end{array}$ & $17^{--}$ & $14^{--}$ & $\begin{array}{r}-- \\
3.2 \\
3.6 \\
-- \\
--\end{array}$ & $\begin{array}{l}-- \\
.70 \\
--\end{array}$ \\
\hline $\begin{array}{l}84-05-24 \\
84-02-02 \\
84-03-07 \\
84-04-13 \\
84-05-31\end{array}$ & $\begin{array}{l}7.3 \\
-- \\
7.6 \\
6.0 \\
6.1\end{array}$ & $\begin{array}{l}640 \\
-- \\
648 \\
650 \\
642\end{array}$ & $\begin{array}{l}160 \\
200 \\
200 \\
180 \\
160\end{array}$ & $\begin{array}{l}200 \\
240 \\
250 \\
210 \\
190\end{array}$ & $\begin{array}{l}0 \\
0 \\
0 \\
0 \\
0\end{array}$ & $\begin{array}{l}16 \\
19 \\
16 \\
11 \\
7.6\end{array}$ & $\begin{array}{r}220 \\
-- \\
--\end{array}$ & $\begin{array}{l}-- \\
63 \\
-- \\
--\end{array}$ & $16^{--}$ & $13^{--}$ & $\begin{array}{l}2.7 \\
3.1 \\
-.- \\
2.7 \\
2.7\end{array}$ & $\begin{array}{l}-\overline{70} \\
-2 \\
--\end{array}$ \\
\hline $\begin{array}{l}84-07-26 \\
84-10-12 \\
84-03-07 \\
84-04-10 \\
84-05-31\end{array}$ & $\begin{array}{l}4.1 \\
4.4 \\
4.4 \\
4.5 \\
2.8\end{array}$ & $\begin{array}{l}645 \\
644 \\
648 \\
637 \\
641\end{array}$ & $\begin{array}{l}170 \\
200 \\
180 \\
180 \\
160\end{array}$ & $\begin{array}{l}210 \\
240 \\
210 \\
210 \\
200\end{array}$ & $\begin{array}{l}0 \\
0 \\
0 \\
0 \\
0\end{array}$ & $\begin{array}{c}11 \\
19 \\
11 \\
13 \\
8.0\end{array}$ & $\begin{array}{r}-- \\
200 \\
-- \\
--\end{array}$ & $\begin{array}{l}-- \\
\overline{59} \\
-- \\
--\end{array}$ & $14=$ & $12^{--}$ & $\begin{array}{r}-- \\
3.1 \\
2.6 \\
2.4 \\
2.1\end{array}$ & $\begin{array}{l}-- \\
.70 \\
--\end{array}$ \\
\hline $\begin{array}{l}84-07-19 \\
84-04-13 \\
84-04-12 \\
84-05-24 \\
84-07-25\end{array}$ & $\begin{array}{l}1.2 \\
8.9 \\
7.6 \\
8.8 \\
5.3\end{array}$ & $\begin{array}{l}646 \\
649 \\
641 \\
641 \\
645\end{array}$ & $\begin{array}{l}180 \\
150 \\
160 \\
150 \\
150\end{array}$ & $\begin{array}{l}220 \\
180 \\
190 \\
180 \\
180\end{array}$ & $\begin{array}{l}0 \\
0 \\
0 \\
0 \\
0\end{array}$ & $\begin{array}{l}14 \\
5.7 \\
9.5 \\
7.2 \\
7.2\end{array}$ & $\begin{array}{l}-- \\
-- \\
-- \\
--\end{array}$ & $\begin{array}{l}-- \\
-- \\
--\end{array}$ & $\begin{array}{l}-- \\
-- \\
--\end{array}$ & $\begin{array}{l}-- \\
-- \\
-- \\
--\end{array}$ & $\begin{array}{l}2.3 \\
1.8 \\
2.2 \\
1.6 \\
--\end{array}$ & $\begin{array}{l}- \\
- \\
-\end{array}$ \\
\hline $\begin{array}{l}84-10-11 \\
84-02-02 \\
84-02-28 \\
84-04-13 \\
84-05-25\end{array}$ & $\begin{array}{r}4.0 \\
.0 \\
-.2 \\
.2 \\
1.1\end{array}$ & $\begin{array}{r}635 \\
-- \\
-- \\
650 \\
642\end{array}$ & $\begin{array}{l}150 \\
250 \\
230 \\
310 \\
320\end{array}$ & $\begin{array}{l}180 \\
300 \\
280 \\
380 \\
390\end{array}$ & $\begin{array}{l}0 \\
0 \\
0 \\
0 \\
0\end{array}$ & $\begin{array}{l}7.2 \\
15 \\
11 \\
30 \\
25\end{array}$ & $\begin{array}{r}220 \\
220 \\
--\end{array}$ & $\begin{array}{l}-- \\
58 \\
58 \\
-- \\
--\end{array}$ & $\begin{array}{l}18^{--} \\
19^{--} \\
--\end{array}$ & $\begin{array}{l}16^{--} \\
16 \\
--\end{array}$ & $\begin{array}{r}2.4 \\
9.8 \\
9.4 \\
13 \\
9.9\end{array}$ & $\begin{array}{l}-- \\
.90 \\
.90 \\
-- \\
--\end{array}$ \\
\hline $\begin{array}{l}84-07-27 \\
84-10-04 \\
85-04-05 \\
85-04-17 \\
85-05-03\end{array}$ & $\begin{array}{l}.7 \\
.6 \\
-. \\
.2 \\
.-\end{array}$ & $\begin{array}{r}643 \\
640 \\
-- \\
636 \\
--\end{array}$ & $\begin{array}{r}510 \\
350 \\
-- \\
520 \\
--\end{array}$ & $\begin{array}{r}620 \\
430 \\
-- \\
630 \\
--\end{array}$ & $\begin{array}{r}0 \\
0 \\
-- \\
0 \\
--\end{array}$ & $\begin{array}{c}156 \\
68 \\
40^{--} \\
--\end{array}$ & $\begin{array}{r}290 \\
\ddot{--} \\
--\end{array}$ & $\begin{array}{l}-- \\
80 \\
-- \\
--\end{array}$ & $21^{--}$ & $21^{--}$ & $\begin{array}{l}50 \\
26 \\
34 \\
25\end{array}$ & $\begin{array}{l}.-- \\
.60 \\
-- \\
--\end{array}$ \\
\hline
\end{tabular}




\begin{tabular}{|c|c|c|c|c|c|c|c|c|c|c|c|c|}
\hline $\begin{array}{c}\text { DATE } \\
\text { OF } \\
\text { SAMPLE }\end{array}$ & $\begin{array}{l}\text { CHLO- } \\
\text { RIDE, } \\
\text { DIS- } \\
\text { SOLVED } \\
\text { (MG/L } \\
\text { AS CL) }\end{array}$ & $\begin{array}{l}\text { SULFATE, } \\
\text { DIS- } \\
\text { SOLVED } \\
\text { (MG/L } \\
\text { AS SO4) }\end{array}$ & $\begin{array}{l}\text { SILICA, } \\
\text { DIS- } \\
\text { SOLVED } \\
\text { (MG/L } \\
\text { AS } \\
\text { SIO2) }\end{array}$ & $\begin{array}{l}\text { CARBON, } \\
\text { ORGANIC, } \\
\text { DISSOLVED } \\
\text { (MG /L } \\
\text { AS C) }\end{array}$ & $\begin{array}{l}\text { METHY- } \\
\text { LENE } \\
\text { BLUE } \\
\text { ACTIVE } \\
\text { SUB- } \\
\text { STANCE } \\
\text { (MG/L) }\end{array}$ & $\begin{array}{c}\text { NITRO- } \\
\text { GEN, } \\
\text { NO2+NO3 } \\
\text { DIS- } \\
\text { SOLVED } \\
\text { (MG/L } \\
\text { AS N) }\end{array}$ & $\begin{array}{l}\text { NITRO- } \\
\text { GEN, } \\
\text { AMMONIA } \\
\text { DIS- } \\
\text { SOLVED } \\
\text { (MG/L } \\
\text { AS N) }\end{array}$ & $\begin{array}{c}\text { NI TRO- } \\
\text { GEN, AM- } \\
\text { MONIA + } \\
\text { ORGANIC } \\
\text { DISSOLVED } \\
\text { (MG/L } \\
\text { AS N) }\end{array}$ & $\begin{array}{l}\text { NITRO- } \\
\text { GEN, } \\
\text { ORGANIC } \\
\text { DIS- } \\
\text { SOLVED } \\
\text { (MG/L } \\
\text { AS N) }\end{array}$ & $\begin{array}{l}\text { NITRO- } \\
\text { GEN, } \\
\text { AMMONIA, } \\
\text { DIS- } \\
\text { SOLVED } \\
\text { (MG/L } \\
\text { AS NH4) }\end{array}$ & $\begin{array}{l}\text { PHOS- } \\
\text { PHORUS, } \\
\text { DIS- } \\
\text { SOLVED } \\
\text { (MG/L } \\
\text { AS P) }\end{array}$ & $\begin{array}{c}\text { IRON, } \\
\text { DIS- } \\
\text { SOLVED } \\
\text { (UG/L } \\
\text { AS FE) }\end{array}$ \\
\hline $\begin{array}{l}84-10-11 \\
84-01-31 \\
84-03-06 \\
84-05-24 \\
84-07-26\end{array}$ & $\begin{array}{r}77^{7.6} \\
-- \\
--\end{array}$ & $\begin{array}{l}26 \\
35 \\
-- \\
-- \\
--\end{array}$ & $\begin{array}{l}19 \\
22 \\
-- \\
-- \\
--\end{array}$ & $\begin{array}{c}2.0 \\
.70 \\
-- \\
--\end{array}$ & $\begin{array}{l}-- \\
=- \\
-- \\
--\end{array}$ & $\begin{array}{l}<0.10 \\
1.6 \\
1.6 \\
1 .-3\end{array}$ & $\begin{array}{r}<0.010 \\
.020 \\
<-010\end{array}$ & $\begin{array}{r}<0.20 \\
-- \\
=- \\
1.0\end{array}$ & $\begin{array}{l}-- \\
=- \\
=- \\
--\end{array}$ & $\begin{array}{r}-- \\
0.03 \\
--\end{array}$ & $\begin{array}{r}0.010 \\
-- \\
-- \\
--\end{array}$ & $\begin{array}{l}10 \\
<3 \\
40 \\
30 \\
--\end{array}$ \\
\hline $\begin{array}{l}84-10-04 \\
84-02-02 \\
84-03-06 \\
84-04-11 \\
84-05-23\end{array}$ & $14^{--}$ & $\begin{array}{l}-- \\
36 \\
-- \\
--\end{array}$ & $\begin{array}{l}-- \\
22 \\
-- \\
--\end{array}$ & $\begin{array}{l}2.9 \\
.70 \\
.50 \\
.80\end{array}$ & $\begin{array}{r}-- \\
-- \\
0.01 \\
--\end{array}$ & $\begin{array}{l}1.6 \\
1.5 \\
1.4 \\
1.7 \\
1.3\end{array}$ & $\begin{array}{r}.010 \\
-1- \\
.020 \\
.030 \\
<.010\end{array}$ & $\begin{array}{l}<.20 \\
-- \\
-7 \\
.70 \\
.20\end{array}$ & $\begin{array}{r}-- \\
\overline{--} \\
0.67 \\
--\end{array}$ & $\begin{array}{l}.01 \\
.03 \\
.04 \\
.-\end{array}$ & $\begin{array}{r}.020 \\
-- \\
-- \\
.060\end{array}$ & $\begin{array}{r}<3 \\
5 \\
20 \\
14 \\
50\end{array}$ \\
\hline $\begin{array}{l}84-07-24 \\
84-10-10 \\
84-02-02 \\
84-03-06 \\
84-04-11\end{array}$ & $14^{--}$ & $\begin{array}{l}-- \\
36 \\
-- \\
--\end{array}$ & $\begin{array}{l}-- \\
23 \\
-- \\
--\end{array}$ & $\begin{array}{c}2 . \overline{-} \\
-\overline{70} \\
--\end{array}$ & $\begin{array}{l}-- \\
=- \\
-- \\
--\end{array}$ & $\begin{array}{l}1.5 \\
1.8 \\
.16 \\
1.6 \\
-.\end{array}$ & $\begin{array}{r}.010 \\
<.010 \\
-0 \\
.050 \\
--\end{array}$ & $\begin{array}{l}.20 \\
.60 \\
-- \\
--\end{array}$ & $\begin{array}{l}.19 \\
-- \\
-- \\
--\end{array}$ & $\begin{array}{l}.01 \\
-- \\
.0 \\
.06 \\
--\end{array}$ & $\begin{array}{r}.- \\
.020 \\
-- \\
--\end{array}$ & $\begin{array}{r}-- \\
7 \\
40 \\
--\end{array}$ \\
\hline $\begin{array}{l}84-05-24 \\
84-02-02 \\
84-03-07 \\
84-04-13 \\
84-05-31\end{array}$ & $13^{--}$ & $\begin{array}{l}-- \\
35 \\
-- \\
--\end{array}$ & $\begin{array}{l}-- \\
21 \\
-- \\
--\end{array}$ & $\begin{array}{l}.80 \\
.- \\
.60 \\
.40 \\
.80\end{array}$ & $\begin{array}{l}-- \\
-- \\
-01 \\
--\end{array}$ & $\begin{array}{l}1.0 \\
1.2 \\
1.4 \\
1.0 \\
.67\end{array}$ & $\begin{array}{r}<.010 \\
-0 \\
.030 \\
.050 \\
<.010\end{array}$ & $\begin{array}{l}.80 \\
-- \\
.2 \\
.20 \\
.60\end{array}$ & $\begin{array}{l}-- \\
-- \\
-\overline{-} \\
.15 \\
--\end{array}$ & $\begin{array}{l}-- \\
.0 \\
.04 \\
.06 \\
--\end{array}$ & $\begin{array}{r}.060 \\
-- \\
-- \\
.010\end{array}$ & $\begin{array}{r}30 \\
7 \\
50 \\
5 \\
40\end{array}$ \\
\hline $\begin{array}{l}84-07-26 \\
84-10-12 \\
84-03-07 \\
84-04-10 \\
84-05-31\end{array}$ & $12^{--}$ & $\begin{array}{l}-- \\
-- \\
26 \\
32 \\
--\end{array}$ & $\begin{array}{l}-- \\
-\overline{19} \\
-- \\
--\end{array}$ & $\begin{array}{l}-- \\
.8 \\
.80 \\
.40 \\
.90\end{array}$ & $\begin{array}{l}-- \\
-- \\
-- \\
--\end{array}$ & $\begin{array}{c}.77 \\
1.1 \\
.67 \\
.70 \\
.13\end{array}$ & $\begin{array}{r}<.010 \\
<.010 \\
.040 \\
.030 \\
<.010\end{array}$ & $\begin{array}{r}.40 \\
.30 \\
-2 \\
<.20 \\
.40\end{array}$ & $\begin{array}{l}-- \\
-- \\
-- \\
--\end{array}$ & $\begin{array}{l}-- \\
.- \\
.05 \\
.04 \\
--\end{array}$ & $\begin{array}{l}-- \\
\overline{-} \\
-- \\
.020\end{array}$ & $\begin{array}{l}-- \\
-- \\
55 \\
18 \\
66\end{array}$ \\
\hline $\begin{array}{l}84-07-19 \\
84-04-13 \\
84-04-12 \\
84-05-24 \\
84-07-25\end{array}$ & $\begin{array}{l}-- \\
-- \\
-- \\
--\end{array}$ & $\begin{array}{l}-- \\
36 \\
37 \\
-- \\
--\end{array}$ & $\begin{array}{l}-- \\
-- \\
-- \\
--\end{array}$ & $\begin{array}{l}1.1 \\
.60 \\
.70 \\
.70 \\
--\end{array}$ & $\begin{array}{l}-- \\
-- \\
=- \\
--\end{array}$ & $\begin{array}{c}.32 \\
.88 \\
.78 \\
1.0 \\
.37\end{array}$ & $\begin{array}{r}.010 \\
.020 \\
.030 \\
<.010 \\
.020\end{array}$ & $\begin{array}{l}.20 \\
.50 \\
.40 \\
.60 \\
.20\end{array}$ & $\begin{array}{l}.19 \\
.48 \\
.37 \\
-- \\
.18\end{array}$ & $\begin{array}{l}.01 \\
.03 \\
.04 \\
-- \\
.03\end{array}$ & $\begin{array}{r}.020 \\
-- \\
-0 \\
.020 \\
--\end{array}$ & $\begin{array}{r}79 \\
3 \\
9 \\
20 \\
--\end{array}$ \\
\hline $\begin{array}{l}84-10-11 \\
84-02-02 \\
84-02-28 \\
84-04-13 \\
84-05-25\end{array}$ & $\begin{array}{l}15^{--} \\
12 \\
-- \\
--\end{array}$ & $\begin{array}{l}-- \\
34 \\
34 \\
21 \\
--\end{array}$ & $\begin{array}{l}-- \\
27 \\
27 \\
-- \\
--\end{array}$ & $\begin{array}{l}-- \\
1.3 \\
1.9 \\
2.0\end{array}$ & $\begin{array}{l}-- \\
=- \\
=- \\
--\end{array}$ & $\begin{array}{r}.49 \\
<.10 \\
<.10 \\
1.4 \\
.78\end{array}$ & $\begin{array}{l}.010 \\
1.30 \\
1.30 \\
1.10\end{array}$ & $\begin{array}{l}.20 \\
-- \\
1.8 \\
1.6\end{array}$ & $\begin{array}{l}.19 \\
-- \\
-- \\
.50 \\
.50\end{array}$ & $\begin{array}{l}.01 \\
-\overline{1} \\
1.7 \\
1.7 \\
1.4\end{array}$ & $\begin{array}{r}-- \\
-- \\
-- \\
.050\end{array}$ & $\begin{array}{l}3800 \\
1800 \\
2900 \\
1700\end{array}$ \\
\hline $\begin{array}{l}84-07-27 \\
84-10-04 \\
85-04-05 \\
85-04-17 \\
85-05-03\end{array}$ & $\begin{array}{c}15 \\
-- \\
--\end{array}$ & $\begin{array}{l}-- \\
24 \\
-- \\
-- \\
--\end{array}$ & $\begin{array}{l}-- \\
24 \\
-- \\
--\end{array}$ & $\begin{array}{r}2.6 \\
4.4 \\
-- \\
-- \\
--\end{array}$ & $\begin{array}{l}-- \\
-- \\
-- \\
--\end{array}$ & $\begin{array}{c}9.0 \\
.57 \\
-- \\
9.6 \\
--\end{array}$ & $\begin{array}{c}.650 \\
1.10 \\
1.40 \\
--\end{array}$ & $\begin{array}{c}1.2 \\
1.4 \\
-- \\
2.7 \\
--\end{array}$ & $\begin{array}{l}.55 \\
.30 \\
-3 \\
1.3 \\
--\end{array}$ & $\begin{array}{c}.84 \\
1.4 \\
1.8 \\
--\end{array}$ & $\begin{array}{r}.030 \\
<.010 \\
-- \\
--\end{array}$ & $\begin{array}{r}120 \\
530 \\
620 \\
-- \\
--\end{array}$ \\
\hline
\end{tabular}


Table 3.--Ground-water quality data, 1984 and 1985--Continued

\begin{tabular}{|c|c|c|c|c|}
\hline $\begin{array}{c}\text { DATE } \\
\text { OF } \\
\text { SAMP LE }\end{array}$ & $\begin{array}{l}\text { IRON, } \\
\text { SUS- } \\
\text { PENDED } \\
\text { RECOV- } \\
\text { ERABLE } \\
\text { (UG/L } \\
\text { AS FE) }\end{array}$ & $\begin{array}{l}\text { IRON, } \\
\text { TOTAL } \\
\text { RECOV- } \\
\text { ERABLE } \\
\text { (UG/L } \\
\text { AS FE) }\end{array}$ & $\begin{array}{l}\text { MANGA- } \\
\text { NESE, } \\
\text { DIS- } \\
\text { SOLVED } \\
\text { (UG/L } \\
\text { AS MN) }\end{array}$ & $\begin{array}{l}\text { ZINC, } \\
\text { DIS- } \\
\text { SOLVED } \\
\text { (UG/L } \\
\text { AS ZN) }\end{array}$ \\
\hline $\begin{array}{l}84-10-11 \\
84-01-31 \\
84-03-06 \\
84-05-24 \\
84-07-26\end{array}$ & $\begin{array}{l}-- \\
-- \\
-- \\
--\end{array}$ & $\begin{array}{l}-- \\
-- \\
50 \\
--\end{array}$ & $\begin{array}{r}1400 \\
<1 \\
<10 \\
<10 \\
--\end{array}$ & $\begin{array}{l}35 \\
-- \\
-- \\
40 \\
--\end{array}$ \\
\hline $\begin{array}{l}84-10-04 \\
84-02-02 \\
84-03-06 \\
84-04-11 \\
84-05-23\end{array}$ & $\frac{--}{--}$ & $\begin{array}{r}-- \\
-- \\
40 \\
<70\end{array}$ & $\begin{array}{r}<1 \\
6 \\
<10 \\
9 \\
<10\end{array}$ & $\begin{array}{r}13 \\
-- \\
-- \\
150 \\
40\end{array}$ \\
\hline $\begin{array}{l}84-07-24 \\
84-10-10 \\
84-02-02 \\
84-03-06 \\
84-04-11\end{array}$ & $\begin{array}{l}-- \\
-- \\
-- \\
--\end{array}$ & $\begin{array}{l}-- \\
-- \\
-- \\
--\end{array}$ & $\begin{array}{r}-- \\
320 \\
2 \\
<10 \\
--\end{array}$ & $\begin{array}{l}30 \\
34 \\
-- \\
-- \\
--\end{array}$ \\
\hline $\begin{array}{l}84-05-24 \\
84-02-02 \\
84-03-07 \\
84-04-13 \\
84-05-31\end{array}$ & $\begin{array}{l}-- \\
-- \\
-- \\
20 \\
--\end{array}$ & $\begin{array}{r}290 \\
-- \\
20 \\
240\end{array}$ & $\begin{array}{r}<10 \\
2 \\
<10 \\
3 \\
10\end{array}$ & $\begin{array}{l}70 \\
-- \\
-- \\
15 \\
50\end{array}$ \\
\hline $\begin{array}{l}84-07-26 \\
84-10-12 \\
84-03-07 \\
84-04-10 \\
84-05-31\end{array}$ & $\frac{--}{--}$ & $\begin{array}{l}-- \\
-- \\
140 \\
250\end{array}$ & $\begin{array}{l}-- \\
240 \\
150 \\
360\end{array}$ & $\begin{array}{r}-- \\
10 \\
63 \\
22\end{array}$ \\
\hline $\begin{array}{l}84-07-19 \\
84-04-13 \\
84-04-12 \\
84-05-24 \\
84-07-25\end{array}$ & $\begin{array}{r}-- \\
230 \\
50 \\
-- \\
--\end{array}$ & $\begin{array}{r}180 \\
230 \\
60 \\
80 \\
--\end{array}$ & $\begin{array}{r}490 \\
<1 \\
6 \\
<10 \\
--\end{array}$ & $\begin{array}{l}15 \\
58 \\
54 \\
40 \\
--\end{array}$ \\
\hline $\begin{array}{l}84-10-11 \\
84-02-02 \\
84-02-28 \\
84-04-13 \\
84-05-25\end{array}$ & $\begin{array}{l}-- \\
-- \\
-- \\
-- \\
--\end{array}$ & $\begin{array}{l}-- \\
-- \\
3200 \\
3600\end{array}$ & $\begin{array}{l}3100 \\
3000 \\
3600 \\
3500\end{array}$ & $\begin{array}{l}40 \\
-- \\
-- \\
90 \\
70\end{array}$ \\
\hline $\begin{array}{l}84-07-27 \\
84-10-04 \\
85-04-05 \\
85-04-17 \\
85-05-03\end{array}$ & $\begin{array}{l}-- \\
-- \\
-- \\
-- \\
--\end{array}$ & $\begin{array}{l}-- \\
-- \\
-- \\
--\end{array}$ & $\begin{array}{r}650 \\
1700 \\
4000 \\
-- \\
--\end{array}$ & $\begin{array}{l}170 \\
160 \\
230 \\
100 \\
--\end{array}$ \\
\hline
\end{tabular}


Table 3.--Ground-water guality data, 1984 and 1985--Continued

\begin{tabular}{|c|c|c|c|c|c|c|c|c|c|c|c|}
\hline $\begin{array}{l}\text { WELL } \\
\text { NUM- } \\
\text { BER }\end{array}$ & $\begin{array}{l}\text { WELL } \\
\text { LOCA- } \\
\text { TION }\end{array}$ & $\begin{array}{c}\text { DATE } \\
\text { OF } \\
\text { SAMPLE }\end{array}$ & TIME & $\begin{array}{l}\text { SPE- } \\
\text { CIFIC } \\
\text { CONDUC- } \\
\text { TANCE* } \\
\text { (UMHOS) }\end{array}$ & $\begin{array}{l}\text { SPE- } \\
\text { CIFIC } \\
\text { CONDUC- } \\
\text { TANCE, } \\
\text { LAB } \\
\text { (UMHOS) }\end{array}$ & $\begin{array}{l}\text { SOLIDS, } \\
\text { SUM OF } \\
\text { CONSTI- } \\
\text { TUENTS, } \\
\text { DIS- } \\
\text { SOLVED } \\
\text { (MG/L) }\end{array}$ & $\begin{array}{l}\text { SOLIDS, } \\
\text { RESIDUE } \\
\text { AT } 180 \\
\text { DEG. C, } \\
\text { DIS- } \\
\text { SOLVED } \\
(M G / L)\end{array}$ & $\begin{array}{l}\text { SOLIDS/ } \\
\text { SPE- } \\
\text { CIFIC } \\
\text { CONDUC- } \\
\text { TANCE, } \\
\text { RATIO }\end{array}$ & $\begin{array}{l}\text { CATION/ } \\
\text { ANION, } \\
\text { PERCENT } \\
\text { DIFFER- } \\
\text { ENCE }\end{array}$ & $\begin{array}{l}\text { TEMPER- } \\
\text { ATURE* } \\
\text { (DEG. C) }\end{array}$ & $\begin{array}{c}\text { PH* } \\
\text { (STAN- } \\
\text { DARD } \\
\text { UNITS) }\end{array}$ \\
\hline \multirow[t]{2}{*}{96.} & $\begin{array}{c}\text { (CONTINUED) } \\
2 S-35 E-33 B A B D 1\end{array}$ & $\begin{array}{l}85-06-14 \\
85-07-24 \\
85-08-09 \\
84-01-31 \\
84-03-07\end{array}$ & $\begin{array}{l}1000 \\
1030 \\
1030 \\
1630 \\
1230\end{array}$ & $\begin{array}{l}818 \\
749 \\
857 \\
467 \\
459\end{array}$ & $\begin{array}{l}827 \\
940 \\
485 \\
463\end{array}$ & $\begin{array}{r}500 \\
-- \\
530 \\
300 \\
--\end{array}$ & $\begin{array}{l}477 \\
-- \\
482 \\
296 \\
268\end{array}$ & $\begin{array}{l}0.58 \\
-5 \\
.56 \\
.63 \\
.58\end{array}$ & $\begin{array}{r}-4.68 \\
-\overline{-} \\
-7.13 \\
-2.74 \\
--\end{array}$ & $\begin{array}{r}11.0 \\
11.0 \\
11.0 \\
8.5 \\
11.5\end{array}$ & $\begin{array}{l}7.0 \\
7.1 \\
6.9 \\
7.3 \\
7.4\end{array}$ \\
\hline & & $\begin{array}{l}84-04-11 \\
84-05-23 \\
84-07-19 \\
84-10-10 \\
85-04-04\end{array}$ & $\begin{array}{l}1010 \\
1240 \\
1130 \\
1300 \\
1630\end{array}$ & $\begin{array}{l}620 \\
572 \\
439 \\
450 \\
671\end{array}$ & $\begin{array}{r}627 \\
544 \\
434 \\
462 \\
--\end{array}$ & $\begin{array}{l}-- \\
-- \\
270 \\
--\end{array}$ & $\begin{array}{r}357 \\
-- \\
-- \\
260\end{array}$ & $\begin{array}{l}.58 \\
-- \\
-5 \\
.58 \\
--\end{array}$ & $\begin{array}{r}-- \\
-4 . \overline{-} \\
-40\end{array}$ & $\begin{array}{l}11.0 \\
11.0 \\
11.0 \\
12.0 \\
10.5\end{array}$ & $\begin{array}{l}7.4 \\
7.3 \\
7.3 \\
7.5 \\
7.3\end{array}$ \\
\hline \multirow[t]{3}{*}{97.} & $2 S-35 E-33 B A D B 1$ & $\begin{array}{l}85-04-17 \\
85-06-13 \\
84-02-02 \\
84-02-28 \\
84-04-13\end{array}$ & $\begin{array}{l}1715 \\
0945 \\
0915 \\
0915 \\
1530\end{array}$ & $\begin{array}{l}797 \\
474 \\
508 \\
507 \\
739\end{array}$ & $\begin{array}{l}-\overline{494} \\
539 \\
515 \\
721\end{array}$ & $\begin{array}{l}-\overline{-} \\
290 \\
330 \\
320 \\
430\end{array}$ & $\begin{array}{l}-\overline{-} \\
273 \\
318 \\
304 \\
380\end{array}$ & $\begin{array}{l}-\overline{-} \\
.58 \\
.63 \\
.60 \\
.58\end{array}$ & $\begin{array}{l}-1.76 \\
-2.59 \\
-2.52 \\
-1.32\end{array}$ & $\begin{array}{l}10.0 \\
10.5 \\
10.0 \\
10.0 \\
10.0\end{array}$ & $\begin{array}{l}7.5 \\
7.3 \\
7.3 \\
7.4 \\
7.0\end{array}$ \\
\hline & & $\begin{array}{l}84-05-25 \\
84-07-27 \\
84-10-04 \\
85-04-05 \\
85-04-18\end{array}$ & $\begin{array}{l}0910 \\
0850 \\
1430 \\
0945 \\
0945\end{array}$ & $\begin{array}{l}564 \\
531 \\
475 \\
709 \\
886\end{array}$ & $\begin{array}{r}571 \\
-- \\
488 \\
-- \\
--\end{array}$ & $\begin{array}{r}-- \\
-- \\
--\end{array}$ & $\begin{array}{r}-- \\
-- \\
273 \\
--\end{array}$ & $\begin{array}{l}-- \\
-- \\
.57 \\
-- \\
--\end{array}$ & $\begin{array}{r}-- \\
-2.48 \\
--\end{array}$ & $\begin{array}{r}10.5 \\
10.5 \\
10.0 \\
-. \\
9.0\end{array}$ & $\begin{array}{r}7.2 \\
7.3 \\
7.4 \\
-.- \\
7.1\end{array}$ \\
\hline & $2 S-35 E-33 B B B C l$ & $\begin{array}{l}85-05-03 \\
85-06-14 \\
85-07-24 \\
85-08-09 \\
84-06-01\end{array}$ & $\begin{array}{l}1200 \\
0915 \\
0930 \\
0930 \\
1200\end{array}$ & $\begin{array}{l}779 \\
576 \\
546 \\
512 \\
499\end{array}$ & $\begin{array}{l}6 \overline{-1} \\
-\overline{-} \\
528 \\
485\end{array}$ & $\begin{array}{l}350 \\
-- \\
310 \\
--\end{array}$ & $\begin{array}{r}33 \overline{2} \\
--\overline{6} \\
306\end{array}$ & $\begin{array}{l}.-- \\
.58 \\
.-- \\
.60 \\
--\end{array}$ & $\begin{array}{r}-1 . \overline{14} \\
-46 \\
--\end{array}$ & $\begin{array}{l}10 . \overline{-} \\
10.0 \\
10.0 \\
11.5\end{array}$ & $\begin{array}{l}-- \\
7.2 \\
7.2 \\
7.2 \\
7.5\end{array}$ \\
\hline 99. & $2 S-35 E-33 B C B D 1$ & $\begin{array}{l}84-07-24 \\
84-10-10 \\
84-02-01 \\
84-07-24 \\
85-06-13\end{array}$ & $\begin{array}{l}1345 \\
1545 \\
1100 \\
1150 \\
1330\end{array}$ & $\begin{array}{l}425 \\
421 \\
373 \\
400 \\
425\end{array}$ & $\begin{array}{l}379 \\
428 \\
374 \\
404 \\
446\end{array}$ & $\begin{array}{r}\overline{--} \\
\overline{-} \\
\overline{-} \\
260\end{array}$ & $\begin{array}{r}24 \overline{3} \\
208 \\
-\overline{4} \\
244\end{array}$ & $\begin{array}{l}-\overline{.} \\
.58 \\
.56 \\
-\overline{.57}\end{array}$ & $\begin{array}{r}-\overline{-} \\
-1.08 \\
-.30\end{array}$ & $\begin{array}{r}11.5 \\
11.0 \\
9.0 \\
9.5 \\
10.0\end{array}$ & $\begin{array}{l}7.2 \\
7.3 \\
7.5 \\
7.2 \\
7.1\end{array}$ \\
\hline 101 & $2 \mathrm{~S}-35 \mathrm{E}-33 \mathrm{BCDCl}$ & $\begin{array}{l}84-02-01 \\
84-03-07 \\
84-04-11 \\
84-05-23 \\
84-07-24\end{array}$ & $\begin{array}{l}0930 \\
0930 \\
0910 \\
0915 \\
0930\end{array}$ & $\begin{array}{l}563 \\
515 \\
680 \\
589 \\
550\end{array}$ & $\begin{array}{r}543 \\
533 \\
688 \\
586 \\
--\end{array}$ & $\begin{array}{c}330 \\
-- \\
-- \\
--\end{array}$ & $\begin{array}{r}-- \\
313 \\
359 \\
-- \\
--\end{array}$ & $\begin{array}{l}.59 \\
.61 \\
.53 \\
-- \\
--\end{array}$ & $\begin{array}{r}-2.30 \\
=- \\
-- \\
--\end{array}$ & $\begin{array}{r}8.5 \\
9.5 \\
10.0 \\
10.5 \\
11.5\end{array}$ & $\begin{array}{l}7.3 \\
7.4 \\
7.4 \\
7.4 \\
7.3\end{array}$ \\
\hline $\begin{array}{l}103 \\
105\end{array}$ & $\begin{array}{l}2 S-35 E-33 C A B C 2 \\
2 S-35 E-33 C B A A 1\end{array}$ & $\begin{array}{l}84-10-10 \\
85-06-13 \\
85-08-09 \\
84-02-01\end{array}$ & $\begin{array}{l}0900 \\
1200 \\
1230 \\
1530\end{array}$ & $\begin{array}{l}486 \\
441 \\
501 \\
411\end{array}$ & $\begin{array}{l}502 \\
458 \\
511 \\
422\end{array}$ & $\begin{array}{l}290 \\
260 \\
300 \\
250\end{array}$ & $\begin{array}{l}293 \\
262 \\
292 \\
260\end{array}$ & $\begin{array}{l}.60 \\
.59 \\
.58 \\
.63\end{array}$ & $\begin{array}{r}-4.09 \\
-7 \\
.42 \\
-3.64\end{array}$ & $\begin{array}{r}11.0 \\
10.5 \\
9.0 \\
9.0\end{array}$ & $\begin{array}{l}7.3 \\
7.3 \\
7.5 \\
8.0\end{array}$ \\
\hline
\end{tabular}


Table 3.--Ground-water quality data, 1984 and $1985--C o n t i n u e d$

\begin{tabular}{|c|c|c|c|c|c|c|c|c|c|c|c|c|}
\hline $\begin{array}{c}\text { DATE } \\
\text { OF } \\
\text { SAMPLE }\end{array}$ & $\begin{array}{c}\text { OXYGEN*, } \\
\text { DIS- } \\
\text { SOLVED } \\
\text { (MG/L) }\end{array}$ & $\begin{array}{l}\text { BARO- } \\
\text { METRIC } \\
\text { PRES- } \\
\text { SURE* } \\
\text { (MM } \\
\text { OF HG) }\end{array}$ & $\begin{array}{l}\text { ALKA- } \\
\text { LINITY* } \\
\text { (MG/L } \\
\text { AS } \\
\text { CACO } 3)\end{array}$ & $\begin{array}{c}\text { BICAR- } \\
\text { BONATE } \\
\text { FET-FLD } \\
\text { (MG/L AS } \\
\text { HCO3) }\end{array}$ & $\begin{array}{c}\text { CAR- } \\
\text { BONATE* } \\
\text { FET-FLD } \\
\text { (MG/L } \\
\text { AS CO3) }\end{array}$ & $\begin{array}{c}\text { CARBON } \\
\text { DIOXIDE, } \\
\text { DIS- } \\
\text { SOLVED } \\
\text { (MG/L } \\
\text { AS CO2) }\end{array}$ & $\begin{array}{c}\text { HARD- } \\
\text { NESS } \\
\text { (MG/L } \\
\text { AS } \\
\text { CACO3) }\end{array}$ & $\begin{array}{l}\text { CALCIUM, } \\
\text { DIS- } \\
\text { SOLVED } \\
\text { (MG/L } \\
\text { AS CA) }\end{array}$ & $\begin{array}{l}\text { MAGNE- } \\
\text { SIUM, } \\
\text { DIS- } \\
\text { SOLVED } \\
\text { (MG/L } \\
\text { AS MG) }\end{array}$ & $\begin{array}{l}\text { SODIUM, } \\
\text { DIS- } \\
\text { SOLVED } \\
\text { (MG/L } \\
\text { AS NA) }\end{array}$ & $\begin{array}{l}\text { POTAS- } \\
\text { SIUM, } \\
\text { DIS- } \\
\text { SOLVED } \\
\text { (MG/L } \\
\text { AS K) }\end{array}$ & $\begin{array}{l}\text { FLUO- } \\
\text { RIDE, } \\
\text { DIS- } \\
\text { SOLVED } \\
\text { (MG/L } \\
\text { AS F) }\end{array}$ \\
\hline $\begin{array}{l}85-06-14 \\
85-07-24 \\
85-08-09 \\
84-01-31 \\
84-03-07\end{array}$ & $\begin{array}{r}0.5 \\
.0 \\
.6 \\
<.1 \\
.4\end{array}$ & $\begin{array}{l}644 \\
642 \\
646 \\
-- \\
648\end{array}$ & $\begin{array}{l}430 \\
380 \\
450 \\
200 \\
190\end{array}$ & $\begin{array}{l}530 \\
460 \\
550 \\
250 \\
240\end{array}$ & $\begin{array}{l}0 \\
0 \\
0 \\
0 \\
0\end{array}$ & $\begin{array}{r}84 \\
58 \\
110 \\
20 \\
15\end{array}$ & $\begin{array}{r}370 \\
-- \\
360 \\
200 \\
--\end{array}$ & $\begin{array}{r}100 \\
100 \\
54 \\
--\end{array}$ & $\begin{array}{l}29 \\
27 \\
16 \\
--\end{array}$ & $\begin{array}{l}24 \\
24 \\
19 \\
--\end{array}$ & $\begin{array}{c}19 \\
23 \\
5.5 \\
--\end{array}$ & $\begin{array}{r}0.60 \\
.60 \\
.80 \\
--\end{array}$ \\
\hline $\begin{array}{l}84-04-11 \\
84-05-23 \\
84-07-19 \\
84-10-10 \\
85-04-04\end{array}$ & $\begin{array}{r}.8 \\
.2 \\
1.4 \\
.1 \\
.6\end{array}$ & $\begin{array}{l}642 \\
641 \\
645 \\
640 \\
639\end{array}$ & $\begin{array}{l}290 \\
250 \\
200 \\
210 \\
330\end{array}$ & $\begin{array}{l}360 \\
310 \\
240 \\
250 \\
400\end{array}$ & $\begin{array}{l}0 \\
0 \\
0 \\
0 \\
0\end{array}$ & $\begin{array}{l}23 \\
25 \\
19 \\
13 \\
32\end{array}$ & $\begin{array}{r}-- \\
-- \\
190 \\
--\end{array}$ & $\begin{array}{l}-- \\
-- \\
50 \\
--\end{array}$ & $15^{--}$ & $17^{--}$ & $\begin{array}{l}6.1 \\
6.3 \\
4.5 \\
5.0 \\
7.5\end{array}$ & $\begin{array}{l}-- \\
-- \\
-\overline{-} \\
.80 \\
--\end{array}$ \\
\hline $\begin{array}{l}85-04-17 \\
85-06-13 \\
84-02-02 \\
84-02-28 \\
84-04-13\end{array}$ & $\begin{array}{l}.2 \\
.2 \\
.1 \\
-. \\
.4\end{array}$ & $\begin{array}{r}635 \\
642 \\
-\overline{--} \\
651\end{array}$ & $\begin{array}{l}410 \\
210 \\
230 \\
220 \\
327\end{array}$ & $\begin{array}{l}500 \\
260 \\
280 \\
270 \\
400\end{array}$ & $\begin{array}{l}0 \\
0 \\
0 \\
0 \\
0\end{array}$ & $\begin{array}{l}25 \\
21 \\
22 \\
17 \\
64\end{array}$ & $\begin{array}{l}-- \\
220 \\
220 \\
210 \\
300\end{array}$ & $\begin{array}{l}-- \\
60 \\
59 \\
57 \\
80\end{array}$ & $\begin{array}{l}16^{--} \\
18 \\
17 \\
24\end{array}$ & $\begin{array}{l}17^{--} \\
26 \\
24 \\
39\end{array}$ & $\begin{array}{l}9.4 \\
1.9 \\
5.8 \\
5.4 \\
6.2\end{array}$ & $\begin{array}{l}.-\overline{1} \\
.70 \\
.80 \\
.80 \\
.70\end{array}$ \\
\hline $\begin{array}{l}84-05-25 \\
84-07-27 \\
84-10-04 \\
85-04-05 \\
85-04-18\end{array}$ & $\begin{array}{l}.5 \\
.5 \\
.3 \\
-.2 \\
.2\end{array}$ & $\begin{array}{r}642 \\
644 \\
640 \\
-- \\
638\end{array}$ & $\begin{array}{r}260 \\
250 \\
220 \\
-\overline{420}\end{array}$ & $\begin{array}{r}310 \\
310 \\
270 \\
-\overline{510}\end{array}$ & $\begin{array}{r}0 \\
0 \\
0 \\
-- \\
0\end{array}$ & $\begin{array}{l}31 \\
25 \\
17 \\
64\end{array}$ & $\begin{array}{r}-- \\
200 \\
-- \\
--\end{array}$ & $\begin{array}{l}-- \\
\overline{52} \\
-- \\
--\end{array}$ & $16^{--}$ & $21^{--}$ & $\begin{array}{l}5.0 \\
5.6 \\
5.4 \\
6.8 \\
7.3\end{array}$ & $\begin{array}{l}-- \\
\overline{-} \\
.80 \\
-- \\
--\end{array}$ \\
\hline $\begin{array}{l}85-05-03 \\
85-06-14 \\
85-07-24 \\
85-08-09 \\
84-06-01\end{array}$ & $\begin{array}{l}-. \\
.4 \\
.2 \\
.4 \\
.3\end{array}$ & $\begin{array}{l}-\overline{643} \\
642 \\
646 \\
646\end{array}$ & $\begin{array}{l}-\overline{280} \\
250 \\
240 \\
220\end{array}$ & $\begin{array}{l}340 \\
300 \\
290 \\
270\end{array}$ & $\begin{array}{l}-- \\
0 \\
0 \\
0 \\
0\end{array}$ & $\begin{array}{l}34^{--} \\
30 \\
29 \\
14\end{array}$ & $\begin{array}{r}-- \\
260 \\
-\overline{-} \\
--\end{array}$ & $\begin{array}{l}-\overline{68} \\
\overline{62} \\
--\end{array}$ & $21^{--}$ & $25^{--}$ & $\begin{array}{r}-- \\
6.5 \\
5.9 \\
3.1\end{array}$ & $\begin{array}{l}.- \\
.80 \\
.80 \\
--\end{array}$ \\
\hline $\begin{array}{l}84-07-24 \\
84-10-10 \\
84-02-01 \\
84-07-24 \\
85-06-13\end{array}$ & $\begin{array}{r}.6 \\
3.0 \\
6.7 \\
.7 \\
.2\end{array}$ & $\begin{array}{r}647 \\
638 \\
-- \\
648 \\
642\end{array}$ & $\begin{array}{r}190 \\
180 \\
-2 \\
180 \\
190\end{array}$ & $\begin{array}{l}240 \\
220 \\
170 \\
220 \\
230\end{array}$ & $\begin{array}{l}0 \\
0 \\
0 \\
0 \\
0\end{array}$ & $\begin{array}{l}24 \\
18 \\
8 \cdot 5 \\
22 \\
29\end{array}$ & $\begin{array}{l}-- \\
190 \\
160 \\
-- \\
200\end{array}$ & $\begin{array}{l}-- \\
56 \\
45 \\
-- \\
56\end{array}$ & $13^{--}-$ & $\begin{array}{l}14^{--} \\
13 \\
16^{--}\end{array}$ & $\begin{array}{l}-\overline{7} \\
2.7 \\
3.1\end{array}$ & $\begin{array}{l}.7 \\
.70 \\
.80 \\
.70\end{array}$ \\
\hline $\begin{array}{l}84-02-01 \\
84-03-07 \\
84-04-11 \\
84-05-23 \\
84-07-24\end{array}$ & $\begin{array}{l}<.1 \\
.3 \\
.5 \\
.3 \\
.3\end{array}$ & $\begin{array}{r}-- \\
645 \\
642 \\
642 \\
--\end{array}$ & $\begin{array}{l}220 \\
210 \\
280 \\
230 \\
200\end{array}$ & $\begin{array}{l}270 \\
260 \\
340 \\
280 \\
250\end{array}$ & $\begin{array}{l}0 \\
0 \\
0 \\
0 \\
0\end{array}$ & $\begin{array}{l}22 \\
16 \\
22 \\
18 \\
20\end{array}$ & $\begin{array}{r}230 \\
-- \\
-- \\
--\end{array}$ & $\begin{array}{l}61 \\
-- \\
-- \\
-- \\
--\end{array}$ & $\begin{array}{r}18 \\
=- \\
-- \\
--\end{array}$ & $\begin{array}{r}25 \\
-- \\
-- \\
--\end{array}$ & $\begin{array}{r}3.8 \\
-.- \\
4.5 \\
4.2 \\
-.2\end{array}$ & $\begin{array}{l}.80 \\
-- \\
-- \\
--\end{array}$ \\
\hline $\begin{array}{l}84-10-10 \\
85-06-13 \\
85-08-09 \\
84-02-01\end{array}$ & $\begin{array}{r}.3 \\
1.8 \\
.4 \\
2.8\end{array}$ & $\begin{array}{r}640 \\
642 \\
646 \\
--\end{array}$ & $\begin{array}{l}190 \\
160 \\
230 \\
180\end{array}$ & $\begin{array}{l}230 \\
200 \\
280 \\
220\end{array}$ & $\begin{array}{l}0 \\
0 \\
0 \\
0\end{array}$ & $\begin{array}{l}18 \\
16 \\
14 \\
3.5\end{array}$ & $\begin{array}{l}200 \\
190 \\
230 \\
190\end{array}$ & $\begin{array}{l}56 \\
53 \\
63 \\
53\end{array}$ & $\begin{array}{l}15 \\
14 \\
18 \\
13\end{array}$ & $\begin{array}{l}20 \\
19 \\
18 \\
14\end{array}$ & $\begin{array}{l}3.7 \\
3.3 \\
4.6 \\
2.6\end{array}$ & $\begin{array}{l}.70 \\
.70 \\
.80 \\
.80\end{array}$ \\
\hline
\end{tabular}


Table 3.--Ground-water quality data, 1984 and 1985--Continued

\begin{tabular}{|c|c|c|c|c|c|c|c|c|c|c|c|c|}
\hline $\begin{array}{c}\text { DATE } \\
\text { OF } \\
\text { SAMPLE }\end{array}$ & $\begin{array}{l}\text { CHLO- } \\
\text { RIDE, } \\
\text { DIS- } \\
\text { SOLVED } \\
\text { (MG/L } \\
\text { AS CL) }\end{array}$ & $\begin{array}{l}\text { SULFATE, } \\
\text { DIS- } \\
\text { SOLVED } \\
\text { (MG/L } \\
\text { AS SO4) }\end{array}$ & $\begin{array}{l}\text { SILICA, } \\
\text { DIS- } \\
\text { SOLVED } \\
\text { (MGG/L } \\
\text { AS } \\
\text { SIO2) }\end{array}$ & $\begin{array}{l}\text { CARBON, } \\
\text { ORGANIC, } \\
\text { DISSOLVED } \\
\text { (MG/L } \\
\text { AS C) }\end{array}$ & $\begin{array}{l}\text { METHY- } \\
\text { LENE } \\
\text { BLUE } \\
\text { ACT IVE } \\
\text { SUB- } \\
\text { STANCE } \\
\text { (MG/L) }\end{array}$ & $\begin{array}{l}\text { NITRO- } \\
\text { GEN, } \\
\text { NO2+NO3 } \\
\text { DIS- } \\
\text { SOLVED } \\
\text { (MG/L } \\
\text { AS N) }\end{array}$ & $\begin{array}{l}\text { NITRO- } \\
\text { GEN, } \\
\text { AMMONIA } \\
\text { DIS- } \\
\text { SOLVED } \\
\text { (MG/L } \\
\text { AS N) }\end{array}$ & $\begin{array}{c}\text { NITRO- } \\
\text { GEN,AM- } \\
\text { MONIA + } \\
\text { ORGANIC } \\
\text { DISSOLVED } \\
\text { (MG/L } \\
\text { AS N) }\end{array}$ & $\begin{array}{c}\text { NITRO- } \\
\text { GEN, } \\
\text { ORGANIC } \\
\text { DIS- } \\
\text { SOLVED } \\
\text { (MG/L } \\
\text { AS N) }\end{array}$ & $\begin{array}{l}\text { NITRO- } \\
\text { GEN, } \\
\text { AMMONIA, } \\
\text { DIS- } \\
\text { SOLVED } \\
\text { (MG/L } \\
\text { AS NH4) }\end{array}$ & $\begin{array}{l}\text { PHOS- } \\
\text { PHORUS, } \\
\text { DIS- } \\
\text { SOLVED } \\
\text { (MG/L } \\
\text { AS P) }\end{array}$ & $\begin{array}{l}\text { IRON, } \\
\text { DIS- } \\
\text { SOLVED } \\
\text { (UG/L } \\
\text { AS FE) }\end{array}$ \\
\hline $\begin{array}{l}85-06-14 \\
85-07-24 \\
85-08-09 \\
84-01-31 \\
84-03-07\end{array}$ & $\begin{array}{l}20 \\
19 \\
14 \\
--\end{array}$ & $\begin{array}{l}21 \\
-25 \\
39 \\
--\end{array}$ & $\begin{array}{l}25 \\
24 \\
23 \\
--\end{array}$ & $\begin{array}{r}2.5 \\
2.0 \\
2.8 \\
.80\end{array}$ & $\begin{array}{l}=- \\
=- \\
=- \\
=\end{array}$ & $\begin{array}{l}3.4 \\
1.2 \\
3.0 \\
<.10 \\
<.10\end{array}$ & $\begin{array}{r}1.10 \\
1.20 \\
1.10 \\
.970\end{array}$ & $\begin{array}{r}1 .- \\
1.5 \\
-- \\
--\end{array}$ & $\begin{array}{r}-\overline{-} \\
0.40 \\
.40 \\
-- \\
--\end{array}$ & $\begin{array}{l}1.4 \\
1.5 \\
1.4 \\
1.2\end{array}$ & $\begin{array}{l}=- \\
=- \\
=- \\
--\end{array}$ & $\begin{array}{r}1000 \\
1500 \\
990 \\
<1400 \\
1100\end{array}$ \\
\hline $\begin{array}{l}84-04-11 \\
84-05-23 \\
84-07-19 \\
84-10-10 \\
85-04-04\end{array}$ & $\begin{array}{c}\overline{-} \\
11 \\
--\end{array}$ & $\begin{array}{l}28 \\
-- \\
-- \\
--\end{array}$ & $\begin{array}{l}-- \\
-- \\
\overline{22} \\
--\end{array}$ & $\begin{array}{l}1.5 \\
1.1 \\
1.6 \\
2.7 \\
--\end{array}$ & $\begin{array}{c}<0.01 \\
-- \\
-\therefore \\
--\end{array}$ & $\begin{array}{l}<.10 \\
<.10 \\
.83 \\
<.10 \\
---\end{array}$ & $\begin{array}{c}1.10 \\
.880 \\
.360 \\
.620 \\
--\end{array}$ & $\begin{array}{l}1.4 \\
2.1 \\
.70 \\
.60 \\
--\end{array}$ & $\begin{array}{r}.30 \\
1.2 \\
.34 \\
-.02 \\
--\end{array}$ & $\begin{array}{l}1.4 \\
1.1 \\
.46 \\
.80 \\
--\end{array}$ & $\begin{array}{r}-- \\
0.050 \\
<.010 \\
.010 \\
--\end{array}$ & $\begin{array}{l}1600 \\
1500 \\
50 \\
2100 \\
5100\end{array}$ \\
\hline $\begin{array}{l}85-04-17 \\
85-06-13 \\
84-02-02 \\
84-02-28 \\
84-04-13\end{array}$ & $\begin{array}{l}14^{--} \\
18 \\
15 \\
21\end{array}$ & $\begin{array}{l}-- \\
30 \\
40 \\
39 \\
32\end{array}$ & $\begin{array}{l}-- \\
20 \\
23 \\
23 \\
23\end{array}$ & $\begin{array}{l}2.3^{--} \\
1.3 \\
1.4\end{array}$ & $\begin{array}{l}-= \\
z- \\
.02\end{array}$ & $\begin{array}{l}<.10 \\
.85 \\
.20 \\
.25 \\
2.0\end{array}$ & $\begin{array}{r}1.10 \\
.420 \\
-0 \\
.770 \\
.660\end{array}$ & $\begin{array}{r}1.7 \\
=- \\
1.2\end{array}$ & $\begin{array}{l}.60 \\
-- \\
-- \\
.44\end{array}$ & $\begin{array}{l}1.4 \\
.54 \\
.99 \\
.99 \\
.85\end{array}$ & $\begin{array}{l}-- \\
=- \\
=- \\
--\end{array}$ & $\begin{array}{r}7000 \\
170 \\
280 \\
78 \\
29\end{array}$ \\
\hline $\begin{array}{l}84-05-25 \\
84-07-27 \\
84-10-04 \\
85-04-05 \\
85-04-18\end{array}$ & $12^{--}$ & $\begin{array}{l}-- \\
- \\
-- \\
--\end{array}$ & $\begin{array}{l}-- \\
\overline{22} \\
-- \\
--\end{array}$ & $\begin{array}{r}1.3 \\
1.4 \\
3.1 \\
-- \\
--\end{array}$ & $\begin{array}{l}=- \\
=- \\
=\end{array}$ & $\begin{array}{r}.28 \\
<.10 \\
<.10 \\
-.- \\
.87\end{array}$ & $\begin{array}{r}.420 \\
.990 \\
.800 \\
- \\
.640\end{array}$ & $\begin{array}{l}1.4 \\
1.1 \\
.70 \\
-1 \\
1.1\end{array}$ & $\begin{array}{r}.98 \\
.11 \\
-.10 \\
.- \\
.46\end{array}$ & $\begin{array}{l}.54 \\
1.3 \\
1.0 \\
-. \\
.82\end{array}$ & $\begin{array}{r}.050 \\
<.010 \\
<.010 \\
-- \\
--\end{array}$ & $\begin{array}{r}80 \\
420 \\
270 \\
130 \\
1400\end{array}$ \\
\hline $\begin{array}{l}85-05-03 \\
85-06-14 \\
85-07-24 \\
85-08-09 \\
84-06-01\end{array}$ & $15^{--}$ & $\begin{array}{l}-- \\
25 \\
-- \\
23 \\
--\end{array}$ & $\begin{array}{l}-\overline{23} \\
\overline{23} \\
--\end{array}$ & $\begin{array}{l}2 .- \\
1.1 \\
1.8 \\
.70\end{array}$ & $\begin{array}{l}-- \\
=- \\
--\end{array}$ & $\begin{aligned} &-- \\
&<.10 \\
&<.10 \\
&<.10 \\
& .17\end{aligned}$ & $\begin{array}{l}.-- \\
.800 \\
.030 \\
.940 \\
.150\end{array}$ & $\begin{array}{l}-- \\
-\overline{1} \\
1.2 \\
.50\end{array}$ & $\begin{array}{l}-- \\
-- \\
1.2 \\
.16 \\
.35\end{array}$ & $\begin{array}{l}--- \\
1.0 \\
.04 \\
1.2 \\
.19\end{array}$ & $\begin{array}{l}-- \\
-- \\
-- \\
.010\end{array}$ & $\begin{array}{r}-- \\
220 \\
1200 \\
810 \\
<10\end{array}$ \\
\hline $\begin{array}{l}84-07-24 \\
84-10-10 \\
84-02-01 \\
84-07-24 \\
85-06-13\end{array}$ & $\begin{array}{l}10^{--} \\
11 \\
11^{--}\end{array}$ & $\begin{array}{l}-- \\
24 \\
32 \\
-- \\
28\end{array}$ & $\begin{array}{l}-- \\
19 \\
17 \\
-- \\
18\end{array}$ & $\begin{array}{l}2 . \overline{6} \\
-\overline{-} \\
.90 \\
2.2\end{array}$ & $\begin{array}{l}-- \\
=- \\
-- \\
--\end{array}$ & $\begin{array}{l}.86 \\
.38 \\
.59 \\
.44 \\
.37\end{array}$ & $\begin{array}{l}.090 \\
.020 \\
.060 \\
.030\end{array}$ & $\begin{array}{r}.50 \\
.20 \\
<.-20 \\
---\end{array}$ & $\begin{array}{l}.41 \\
.18 \\
-- \\
-- \\
--\end{array}$ & $\begin{array}{l}.12 \\
.03 \\
.08 \\
.04\end{array}$ & $\begin{array}{r}.0 \\
.010 \\
-- \\
--\end{array}$ & $\begin{array}{r}-- \\
3 \\
5 \\
7 \\
<3\end{array}$ \\
\hline $\begin{array}{l}84-02-01 \\
84-03-07 \\
84-04-11 \\
84-05-23 \\
84-07-24\end{array}$ & $\begin{array}{r}26 \\
=- \\
=- \\
--\end{array}$ & $\begin{array}{l}37 \\
-- \\
30 \\
--\end{array}$ & $\begin{array}{l}21 \\
=- \\
=- \\
--\end{array}$ & $\begin{array}{l}1 .- \\
1.1 \\
1.2 \\
1.2 \\
1.2\end{array}$ & $\begin{array}{l}-- \\
\overline{01} \\
\overline{--}\end{array}$ & $\begin{array}{r}.31 \\
<.10 \\
.44 \\
.31 \\
.83\end{array}$ & $\begin{array}{l}-310 \\
.380 \\
.180 \\
.060\end{array}$ & $\begin{array}{l}-- \\
. \overline{-} \\
.70 \\
.80 \\
.20\end{array}$ & $\begin{array}{l}-- \\
-\overline{4} \\
.42 \\
.62 \\
.14\end{array}$ & $\begin{array}{l}-.- \\
.40 \\
.36 \\
.23 \\
.08\end{array}$ & $\begin{array}{c}-- \\
-- \\
.040 \\
--\end{array}$ & $\begin{array}{r}97 \\
190 \\
16 \\
15 \\
8\end{array}$ \\
\hline $\begin{array}{l}84-10-10 \\
85-06-13 \\
85-08-09 \\
84-02-01\end{array}$ & $\begin{array}{l}27 \\
28 \\
12 \\
12\end{array}$ & $\begin{array}{l}38 \\
29 \\
26 \\
30\end{array}$ & $\begin{array}{l}20 \\
18 \\
22 \\
19\end{array}$ & $\begin{array}{l}2.8 \\
2.3 \\
1.8 \\
--\end{array}$ & $\begin{array}{l}=- \\
=- \\
=-\end{array}$ & $\begin{array}{r}.53 \\
.62 \\
.15 \\
1.5\end{array}$ & $\begin{array}{l}.060 \\
.050 \\
.340 \\
--\end{array}$ & $\begin{array}{l}.30 \\
.60 \\
--\end{array}$ & $\begin{array}{l}.24 \\
.26 \\
--\end{array}$ & $\begin{array}{l}.08 \\
.06 \\
.44 \\
.--\end{array}$ & $\begin{array}{r}.010 \\
-- \\
--\end{array}$ & $\begin{array}{r}14 \\
22 \\
680 \\
8\end{array}$ \\
\hline
\end{tabular}


Table 3.--Ground-water quality data, 1984 and $1985--$ Continued

\begin{tabular}{cclll}
\hline & IRON, & & & \\
& SUS- & IRON, & MANGA- & \\
& PENDED & TOTAL & NESE, & 2INC, \\
& RECOV- & RECOV- & DIS- & DIS- \\
DATE & ERABLE & ERABLE & SOLVED & SOLVED \\
OF & (UG/L & (UG/L & (UG/L & (UG/L \\
SAMPLE & AS FE) & AS FE) & AS MN) & AS ZN) \\
& & & &
\end{tabular}

\begin{tabular}{|c|c|c|c|c|}
\hline $\begin{array}{l}85-06-14 \\
85-07-24 \\
85-08-09 \\
84-01-31 \\
84-03-07\end{array}$ & $\begin{array}{l}-- \\
-- \\
-- \\
-- \\
--\end{array}$ & $\begin{array}{l}-- \\
-- \\
-- \\
--\end{array}$ & $\begin{array}{l}2900 \\
2600 \\
2500 \\
1900 \\
1700\end{array}$ & $\begin{array}{r}74 \\
94 \\
130 \\
-- \\
--\end{array}$ \\
\hline $\begin{array}{l}84-04-11 \\
84-05-23 \\
84-07-19 \\
84-10-10 \\
85-04-04\end{array}$ & $\begin{array}{r}100 \\
-- \\
-- \\
-- \\
--\end{array}$ & $\begin{array}{r}1700 \\
1600 \\
20 \\
-- \\
--\end{array}$ & $\begin{array}{r}2400 \\
2200 \\
680 \\
1600 \\
2800\end{array}$ & $\begin{array}{r}200 \\
150 \\
70 \\
120 \\
18\end{array}$ \\
\hline $\begin{array}{l}85-04-17 \\
85-06-13 \\
84-02-02 \\
84-02-28 \\
84-04-13\end{array}$ & $\begin{array}{l}-- \\
-- \\
-- \\
-- \\
50\end{array}$ & $\begin{array}{l}-- \\
-- \\
-- \\
-- \\
80\end{array}$ & $\begin{array}{l}3600 \\
1200 \\
2000 \\
1900 \\
1900\end{array}$ & $\begin{array}{r}<7 \\
44 \\
-- \\
-- \\
110\end{array}$ \\
\hline $\begin{array}{l}84-05-25 \\
84-07-27 \\
84-10-04 \\
85-04-05 \\
85-04-18\end{array}$ & $\begin{array}{l}-- \\
-- \\
-- \\
-- \\
--\end{array}$ & $\begin{array}{c}190 \\
-- \\
-- \\
-- \\
--\end{array}$ & $\begin{array}{l}1000 \\
2000 \\
1800 \\
2200 \\
2700\end{array}$ & $\begin{array}{r}110 \\
92 \\
89 \\
140 \\
99\end{array}$ \\
\hline $\begin{array}{l}85-05-03 \\
85-06-14 \\
85-07-24 \\
85-08-09 \\
84-06-01\end{array}$ & $\begin{array}{l}-- \\
-- \\
-- \\
-- \\
--\end{array}$ & $\begin{array}{l}-- \\
-- \\
-- \\
-- \\
<10\end{array}$ & $\begin{array}{r}1900 \\
1900 \\
1900 \\
350\end{array}$ & $\begin{array}{l}-- \\
72 \\
72 \\
74 \\
50\end{array}$ \\
\hline $\begin{array}{l}84-07-24 \\
84-10-10 \\
84-02-01 \\
84-07-24 \\
85-06-13\end{array}$ & $\begin{array}{l}-- \\
-- \\
-- \\
-- \\
--\end{array}$ & $\begin{array}{l}-- \\
-- \\
-- \\
-- \\
--\end{array}$ & $\begin{array}{r}-- \\
39 \\
1 \\
2 \\
23\end{array}$ & $\begin{array}{l}-- \\
21 \\
-- \\
45 \\
28\end{array}$ \\
\hline $\begin{array}{l}84-02-01 \\
84-03-07 \\
84-04-11 \\
84-05-23 \\
84-07-24\end{array}$ & $\begin{array}{l}-- \\
-- \\
-- \\
-- \\
--\end{array}$ & $\begin{array}{r}-- \\
-- \\
<10 \\
50 \\
--\end{array}$ & $\begin{array}{r}1100 \\
1300 \\
1400 \\
1000 \\
230\end{array}$ & $\begin{array}{l}-- \\
-- \\
130 \\
160 \\
120\end{array}$ \\
\hline $\begin{array}{l}84-10-10 \\
85-06-13 \\
85-08-09 \\
84-02-01\end{array}$ & $\begin{array}{l}-- \\
-- \\
-- \\
--\end{array}$ & $\begin{array}{l}-- \\
-- \\
--\end{array}$ & $\begin{array}{r}340 \\
170 \\
1300 \\
4\end{array}$ & $\begin{array}{r}130 \\
110 \\
21 \\
--\end{array}$ \\
\hline
\end{tabular}


Table 3.--Ground-water quality data, 1984 and 1985--Continued

\begin{tabular}{|c|c|c|c|c|c|c|c|c|c|c|c|}
\hline $\begin{array}{l}\text { WELL } \\
\text { NUM- } \\
\text { BER }\end{array}$ & $\begin{array}{l}\text { WELL } \\
\text { LOCA- } \\
\text { TION }\end{array}$ & $\begin{array}{l}\text { DATE } \\
\text { OF } \\
\text { SAMPLE }\end{array}$ & TIME & $\begin{array}{l}\text { SPE- } \\
\text { CIFIC } \\
\text { CONDUC- } \\
\text { TANCE } \\
\text { (UMHOS) }\end{array}$ & $\begin{array}{l}\text { SPE- } \\
\text { CIFIC } \\
\text { CONDUC- } \\
\text { TANCE, } \\
\text { LAB } \\
\text { (UMHOS) }\end{array}$ & $\begin{array}{l}\text { SOLIDS, } \\
\text { SUM OF } \\
\text { CONSTI- } \\
\text { TUENTS, } \\
\text { DIS- } \\
\text { SOLVED } \\
\text { (MG/L) }\end{array}$ & $\begin{array}{l}\text { SOLIDS, } \\
\text { RESIDUE } \\
\text { AT } 180 \\
\text { DEG. C, } \\
\text { DIS- } \\
\text { SOLVED } \\
\text { (MG/L) }\end{array}$ & $\begin{array}{l}\text { SOLIDS/ } \\
\text { SPE- } \\
\text { CIFIC } \\
\text { CONDUC- } \\
\text { TANCE, } \\
\text { RATIO }\end{array}$ & $\begin{array}{l}\text { CATION/ } \\
\text { ANION, } \\
\text { PERCENT } \\
\text { DIFFER- } \\
\text { ENCE }\end{array}$ & $\begin{array}{l}\text { TEMPER- } \\
\text { ATURE` } \\
\text { (DEG. C) }\end{array}$ & $\begin{array}{l}\text { PH* } \\
\text { (STAN- } \\
\text { DARD } \\
\text { UNITS) }\end{array}$ \\
\hline 105 & (CONTINUED) & $\begin{array}{l}84-03-09 \\
84-04-11 \\
84-05-25 \\
84-10-10 \\
85-06-13\end{array}$ & $\begin{array}{l}1000 \\
1200 \\
1520 \\
1500 \\
1045\end{array}$ & $\begin{array}{l}390 \\
406 \\
414 \\
412 \\
405\end{array}$ & $\begin{array}{l}410 \\
415 \\
411 \\
420 \\
420\end{array}$ & $\begin{array}{l}-- \\
\overline{--} \\
260\end{array}$ & $\begin{array}{r}239 \\
238 \\
-- \\
-\overline{244}\end{array}$ & $\begin{array}{r}0.61 \\
.59 \\
-- \\
.- \\
.60\end{array}$ & $\begin{array}{r}\overline{--} \\
\overline{--} \\
2 . \overline{61}\end{array}$ & $\begin{array}{r}9.0 \\
9.5 \\
9.5 \\
12.0 \\
10.0\end{array}$ & $\begin{array}{l}8.0 \\
7.9 \\
7.8 \\
7.8 \\
7.9\end{array}$ \\
\hline $\begin{array}{l}106 . \\
107\end{array}$ & $\begin{array}{l}2 S-35 E-33 C B B A l \\
2 S-35 E-33 C B B C l\end{array}$ & $\begin{array}{l}84-05-22 \\
84-07-18 \\
84-10-10 \\
84-01-31 \\
84-03-06\end{array}$ & $\begin{array}{l}1840 \\
1955 \\
1750 \\
1300 \\
0910\end{array}$ & $\begin{array}{l}504 \\
425 \\
427 \\
431 \\
409\end{array}$ & $\begin{array}{l}499 \\
428 \\
438 \\
418 \\
420\end{array}$ & $\begin{array}{r}-- \\
-- \\
250 \\
--\end{array}$ & $\begin{array}{r}-- \\
-- \\
240 \\
240\end{array}$ & $\begin{array}{l}-- \\
-- \\
-\overline{-} \\
.56 \\
.59\end{array}$ & $\begin{array}{r}-- \\
-. \\
-.89 \\
--\end{array}$ & $\begin{array}{r}9.0 \\
10.0 \\
11.0 \\
8.5 \\
9.0\end{array}$ & $\begin{array}{l}7.3 \\
7.5 \\
7.4 \\
7.7 \\
7.3\end{array}$ \\
\hline 108 & $2 S-35 E-33 C B C B 1$ & $\begin{array}{l}84-04-13 \\
84-05-24 \\
84-07-26 \\
84-10-04 \\
84-05-23\end{array}$ & $\begin{array}{l}1045 \\
1300 \\
1000 \\
1200 \\
1720\end{array}$ & $\begin{array}{l}490 \\
597 \\
511 \\
505 \\
571\end{array}$ & $\begin{array}{l}483 \\
585 \\
478 \\
519 \\
559\end{array}$ & $\begin{array}{l}-- \\
=- \\
=- \\
--\end{array}$ & $\begin{array}{r}266 \\
-- \\
-- \\
-- \\
--\end{array}$ & $\begin{array}{l}.54 \\
-- \\
-- \\
--\end{array}$ & $\begin{array}{l}-- \\
\overline{--} \\
\overline{--}\end{array}$ & $\begin{array}{r}9.0 \\
9.5 \\
9.5 \\
9.5 \\
10.0\end{array}$ & $\begin{array}{l}7.2 \\
7.2 \\
7.3 \\
7.3 \\
7.2\end{array}$ \\
\hline 109 & $2 S-35 E-33$ CCAAI & $\begin{array}{l}84-07-25 \\
84-10-10 \\
84-04-12 \\
84-05-23 \\
84-07-25\end{array}$ & $\begin{array}{l}1655 \\
1645 \\
1210 \\
1600 \\
1600\end{array}$ & $\begin{array}{l}577 \\
557 \\
520 \\
535 \\
524\end{array}$ & $\begin{array}{l}537 \\
569 \\
529 \\
523 \\
519\end{array}$ & $\begin{array}{l}=- \\
=- \\
=-\end{array}$ & $\begin{array}{r}-- \\
-- \\
293 \\
-- \\
--\end{array}$ & $\begin{array}{l}-- \\
.- \\
.56 \\
-- \\
--\end{array}$ & $\begin{array}{l}-- \\
-- \\
-- \\
--\end{array}$ & $\begin{array}{r}10.5 \\
10.0 \\
8.0 \\
10.5 \\
13.5\end{array}$ & $\begin{array}{l}7.2 \\
7.3 \\
7.0 \\
7.2 \\
7.3\end{array}$ \\
\hline 110 & $2 S-35 E-33 C C B B 1$ & $\begin{array}{l}84-10-11 \\
84-04-12 \\
84-05-24 \\
84-07-26 \\
84-10-11\end{array}$ & $\begin{array}{l}1520 \\
1755 \\
1845 \\
1415 \\
1700\end{array}$ & $\begin{array}{l}532 \\
505 \\
422 \\
484 \\
487\end{array}$ & $\begin{array}{l}545 \\
511 \\
420 \\
463 \\
501\end{array}$ & $\begin{array}{l}-- \\
=- \\
=- \\
--\end{array}$ & $\begin{array}{r}-- \\
304 \\
-- \\
--\end{array}$ & $\begin{array}{l}.- \\
.60 \\
-- \\
-- \\
--\end{array}$ & $\begin{array}{l}-- \\
-- \\
-- \\
--\end{array}$ & $\begin{array}{l}12.5 \\
11.5 \\
11.5 \\
13.5 \\
12.0\end{array}$ & $\begin{array}{l}7.3 \\
7.3 \\
7.3 \\
7.3 \\
7.3\end{array}$ \\
\hline $\begin{array}{l}\text { M2. } \\
\text { M12. } \\
112 .\end{array}$ & $\begin{array}{l}2 S-35 E-33 D A B C 1 \\
2 S-35 E-33 D A C B 1 \\
2 S-35 E-33 D B B A 1\end{array}$ & $\begin{array}{l}85-08-08 \\
85-08-08 \\
84-02-02 \\
84-02-28 \\
84-04-13\end{array}$ & $\begin{array}{l}1630 \\
1800 \\
1115 \\
1108 \\
1715\end{array}$ & $\begin{array}{l}326 \\
336 \\
518 \\
531 \\
680\end{array}$ & $\begin{array}{l}348 \\
501 \\
537 \\
646\end{array}$ & $\begin{array}{l}206 \\
310 \\
320 \\
380\end{array}$ & $\begin{array}{r}-- \\
199 \\
307 \\
306 \\
--\end{array}$ & $\begin{array}{l}.- \\
.59 \\
.59 \\
.58 \\
.56\end{array}$ & $\begin{array}{r}-\overline{.71} \\
-4.10 \\
-1.42 \\
-3.51\end{array}$ & $\begin{array}{r}13.0 \\
6.5 \\
9.5 \\
10.5 \\
8.0\end{array}$ & $\begin{array}{l}7.8 \\
7.8 \\
7.5 \\
7.5 \\
7.3\end{array}$ \\
\hline & & $\begin{array}{l}84-05-25 \\
84-07-27 \\
84-10-04 \\
85-04-05 \\
85-04-18\end{array}$ & $\begin{array}{l}1030 \\
1005 \\
1545 \\
1015 \\
1100\end{array}$ & $\begin{array}{l}536 \\
409 \\
449 \\
783 \\
765\end{array}$ & $\begin{array}{r}511 \\
407 \\
434 \\
-- \\
--\end{array}$ & $\begin{array}{r}-- \\
-- \\
\overline{--} \\
--\end{array}$ & $\begin{array}{r}-- \\
-- \\
259 \\
-- \\
--\end{array}$ & $\begin{array}{l}-- \\
-\overline{-} \\
.58 \\
-- \\
--\end{array}$ & $\begin{array}{r}-- \\
-3.91 \\
--\end{array}$ & $\begin{array}{l}9.0 \\
9.0 \\
9.0 \\
-- \\
8.0\end{array}$ & $\begin{array}{l}7.2 \\
7.3 \\
7.4 \\
-- \\
7.1\end{array}$ \\
\hline M3. & $2 S-35 E-33 D D B A 1$ & $\begin{array}{l}85-05-03 \\
85-06-14 \\
85-07-24 \\
85-08-09 \\
85-08-07\end{array}$ & $\begin{array}{l}1200 \\
1030 \\
1145 \\
1130 \\
1015\end{array}$ & $\begin{array}{l}702 \\
618 \\
580 \\
604 \\
440\end{array}$ & $\begin{array}{l}6 \overline{0} \\
\overline{--} \\
459\end{array}$ & $\begin{array}{l}3 \overline{--} \\
\overline{370} \\
270\end{array}$ & $\begin{array}{r}-- \\
351 \\
\overline{--} \\
256\end{array}$ & $\begin{array}{l}.- \\
.57 \\
-\overline{-} \\
.61 \\
.58\end{array}$ & $\begin{array}{r}-\overline{-} \\
-5.76 \\
-4.36 \\
-3.59\end{array}$ & $\begin{array}{l}-\overline{-} \\
8.5 \\
9.0 \\
9.0 \\
8.0\end{array}$ & $\begin{array}{l}-- \\
7.2 \\
7.3 \\
7.2 \\
7.2\end{array}$ \\
\hline
\end{tabular}


Table 3.--Ground-water quality data, 1984 and 1985--Continued

\begin{tabular}{|c|c|c|c|c|c|c|c|c|c|c|c|c|}
\hline $\begin{array}{c}\text { DATE } \\
\text { OF } \\
\text { SAMPLE }\end{array}$ & $\begin{array}{c}\text { OXYGEN*, } \\
\text { DIS- } \\
\text { SOLVED } \\
\text { (MG/L) }\end{array}$ & $\begin{array}{l}\text { BARO- } \\
\text { METRIC } \\
\text { PRES- } \\
\text { SURE* } \\
\text { (MM } \\
\text { OF HG) }\end{array}$ & $\begin{array}{l}\text { ALKA- } \\
\text { LINITY* } \\
\text { (MG /L } \\
\text { AS } \\
\text { CACO3) }\end{array}$ & $\begin{array}{c}\text { BICAR- } \\
\text { BONATE* } \\
\text { FET-FLD } \\
\text { (MG /L AS } \\
\text { HCO } 3)\end{array}$ & $\begin{array}{c}\text { CAR- } \\
\text { BONATE* } \\
\text { FET-FLD } \\
\text { (MG/L } \\
\text { AS CO3) }\end{array}$ & $\begin{array}{c}\text { CARBON } \\
\text { DIOXIDE, } \\
\text { DIS- } \\
\text { SOLVED } \\
\text { (MG/L } \\
\text { AS CO2) }\end{array}$ & $\begin{array}{l}\text { HARD- } \\
\text { NESS } \\
\text { (MG/L } \\
\text { AS } \\
\text { CACO3) }\end{array}$ & $\begin{array}{l}\text { CALCIUM, } \\
\text { DIS- } \\
\text { SOLVED } \\
\text { (MG/L } \\
\text { AS CA) }\end{array}$ & $\begin{array}{l}\text { MAGNE- } \\
\text { SIUM, } \\
\text { DIS- } \\
\text { SOLVED } \\
\text { (MG/L } \\
\text { AS MG) }\end{array}$ & $\begin{array}{l}\text { SODIUM, } \\
\text { DIS- } \\
\text { SOLVED } \\
\text { (MG/L } \\
\text { AS NA) }\end{array}$ & $\begin{array}{l}\text { POTAS- } \\
\text { SIUM, } \\
\text { DIS- } \\
\text { SOLVED } \\
\text { (MG/L } \\
\text { AS K) }\end{array}$ & $\begin{array}{l}\text { FLUO- } \\
\text { RIDE, } \\
\text { DIS- } \\
\text { SOLVED } \\
\text { (MG /L } \\
\text { AS F) }\end{array}$ \\
\hline $\begin{array}{l}84-03-09 \\
84-04-11 \\
84-05-25 \\
84-10-10 \\
85-06-13\end{array}$ & $\begin{array}{l}1.3 \\
1.7 \\
1.5 \\
.5 \\
3.1\end{array}$ & $\begin{array}{l}647 \\
643 \\
642 \\
639 \\
642\end{array}$ & $\begin{array}{l}170 \\
180 \\
180 \\
180 \\
170\end{array}$ & $\begin{array}{l}210 \\
220 \\
220 \\
220 \\
210\end{array}$ & $\begin{array}{l}0 \\
0 \\
0 \\
0 \\
0\end{array}$ & $\begin{array}{l}3.3 \\
4.4 \\
5.5 \\
5.5 \\
4.2\end{array}$ & $\begin{array}{l}=- \\
\overline{-} \\
190\end{array}$ & $\begin{array}{l}=- \\
=- \\
-- \\
54\end{array}$ & $\begin{array}{l}=- \\
=- \\
=-\end{array}$ & $\begin{array}{l}=- \\
=-\end{array}$ & $\begin{array}{l}--- \\
2.5 \\
2.3 \\
2.6 \\
5.5\end{array}$ & $\begin{array}{l}=- \\
=- \\
0 . \overline{80}\end{array}$ \\
\hline $\begin{array}{l}84-05-22 \\
84-07-18 \\
84-10-10 \\
84-01-31 \\
84-03-06\end{array}$ & $\begin{array}{r}.2 \\
1.0 \\
.3 \\
<.1 \\
.2\end{array}$ & $\begin{array}{l}644 \\
640 \\
637 \\
-- \\
648\end{array}$ & $\begin{array}{l}170 \\
190 \\
180 \\
160 \\
160\end{array}$ & $\begin{array}{l}200 \\
230 \\
220 \\
200 \\
200\end{array}$ & $\begin{array}{l}0 \\
0 \\
0 \\
0 \\
0\end{array}$ & $\begin{array}{l}16 \\
12 \\
14 \\
6.3 \\
16\end{array}$ & $\begin{array}{r}-- \\
-- \\
180 \\
--\end{array}$ & $\begin{array}{l}-- \\
=- \\
51 \\
--\end{array}$ & $12^{--}$ & $14^{--}$ & $\begin{array}{r}3.1 \\
2.9 \\
2.8 \\
3.1 \\
-.\end{array}$ & $\begin{array}{l}-- \\
=- \\
.80 \\
--\end{array}$ \\
\hline $\begin{array}{l}84-04-13 \\
84-05-24 \\
84-07-26 \\
84-10-04 \\
84-05-23\end{array}$ & $\begin{array}{r}1.4 \\
.4 \\
.5 \\
.3 \\
1.4\end{array}$ & $\begin{array}{l}650 \\
641 \\
645 \\
640 \\
641\end{array}$ & $\begin{array}{l}160 \\
200 \\
180 \\
200 \\
200\end{array}$ & $\begin{array}{l}200 \\
240 \\
220 \\
240 \\
240\end{array}$ & $\begin{array}{l}0 \\
0 \\
0 \\
0 \\
0\end{array}$ & $\begin{array}{l}20 \\
30 \\
18 \\
19 \\
24\end{array}$ & $\begin{array}{l}=- \\
=- \\
=-\end{array}$ & $\begin{array}{l}=- \\
=- \\
=- \\
--\end{array}$ & $\begin{array}{l}-- \\
-- \\
-- \\
--\end{array}$ & $\begin{array}{l}-- \\
=- \\
=-\end{array}$ & $\begin{array}{l}3.4 \\
4.1 \\
-.- \\
3.6 \\
3.4\end{array}$ & $\begin{array}{l}=- \\
=- \\
=-\end{array}$ \\
\hline $\begin{array}{l}84-07-25 \\
84-10-10 \\
84-04-12 \\
84-05-23 \\
84-07-25\end{array}$ & $\begin{array}{l}2.2 \\
2.4 \\
2.6 \\
4.2 \\
4.6\end{array}$ & $\begin{array}{l}645 \\
638 \\
642 \\
641 \\
645\end{array}$ & $\begin{array}{l}200 \\
210 \\
210 \\
220 \\
230\end{array}$ & $\begin{array}{l}250 \\
250 \\
250 \\
270 \\
280\end{array}$ & $\begin{array}{l}0 \\
0 \\
0 \\
0 \\
0\end{array}$ & $\begin{array}{l}25 \\
20 \\
40 \\
27 \\
22\end{array}$ & $\begin{array}{l}=- \\
=- \\
=- \\
--\end{array}$ & $\begin{array}{l}=- \\
=- \\
=- \\
--\end{array}$ & $\begin{array}{l}-- \\
-- \\
=- \\
--\end{array}$ & $\begin{array}{l}-- \\
=- \\
-- \\
--\end{array}$ & $\begin{array}{r}-- \\
3.9 \\
3.1 \\
3.0 \\
--\end{array}$ & $\begin{array}{l}-- \\
=- \\
--\end{array}$ \\
\hline $\begin{array}{l}84-10-11 \\
84-04-12 \\
84-05-24 \\
84-07-26 \\
84-10-11\end{array}$ & $\begin{array}{l}4.8 \\
4.4 \\
6.1 \\
5.3 \\
5.5\end{array}$ & $\begin{array}{l}635 \\
642 \\
640 \\
645 \\
637\end{array}$ & $\begin{array}{l}230 \\
220 \\
170 \\
210 \\
210\end{array}$ & $\begin{array}{l}280 \\
260 \\
210 \\
260 \\
260\end{array}$ & $\begin{array}{l}0 \\
0 \\
0 \\
0 \\
0\end{array}$ & $\begin{array}{l}22 \\
21 \\
17 \\
21 \\
21\end{array}$ & $\begin{array}{l}=- \\
=- \\
=- \\
--\end{array}$ & $\begin{array}{l}-- \\
-- \\
=- \\
--\end{array}$ & $\begin{array}{l}-- \\
=- \\
=- \\
=-\end{array}$ & $\begin{array}{l}=- \\
=- \\
=- \\
--\end{array}$ & $\begin{array}{l}3.5 \\
3.2 \\
3.1 \\
-- \\
3.4\end{array}$ & $\begin{array}{l}=- \\
=- \\
=-\end{array}$ \\
\hline $\begin{array}{l}85-08-08 \\
85-08-08 \\
84-02-02 \\
84-02-28 \\
84-04-13\end{array}$ & $\begin{array}{r}2.0 \\
5.9 \\
-- \\
-. \\
.2\end{array}$ & $\begin{array}{r}641 \\
641 \\
-- \\
650\end{array}$ & $\begin{array}{l}130 \\
230 \\
240 \\
310\end{array}$ & $\begin{array}{l}170 \\
280 \\
290 \\
370\end{array}$ & $\begin{array}{c}-- \\
0 \\
0 \\
0 \\
0\end{array}$ & $\begin{array}{l}4.0 \\
14 \\
15 \\
29\end{array}$ & $\begin{array}{l}-\overline{150} \\
200 \\
230 \\
270\end{array}$ & $\begin{array}{l}-- \\
42 \\
53 \\
59 \\
70\end{array}$ & $\begin{array}{l}111^{--} \\
17 \\
19 \\
24\end{array}$ & $\begin{array}{l}13^{--} \\
15 \\
16 \\
18\end{array}$ & $\begin{array}{l}2 .- \\
15 \\
15 \\
14\end{array}$ & $\begin{array}{l}.8 \\
.80 \\
.90 \\
.90 \\
.90\end{array}$ \\
\hline $\begin{array}{l}84-05-25 \\
84-07-27 \\
84-10-04 \\
85-04-05 \\
85-04-18\end{array}$ & $\begin{array}{l}.1 \\
.6 \\
.1 \\
-.2 \\
.2\end{array}$ & $\begin{array}{r}642 \\
644 \\
640 \\
-- \\
638\end{array}$ & $\begin{array}{r}280 \\
210 \\
200 \\
-- \\
400\end{array}$ & $\begin{array}{l}340 \\
250 \\
240 \\
-- \\
490\end{array}$ & $\begin{array}{r}0 \\
0 \\
0 \\
-- \\
0\end{array}$ & $\begin{array}{l}34 \\
20 \\
15 \\
62\end{array}$ & $\begin{array}{r}-- \\
170 \\
-- \\
--\end{array}$ & $\begin{array}{l}-- \\
\overline{44} \\
-- \\
--\end{array}$ & $14^{--}$ & $12^{--}$ & $\begin{array}{l}19 \\
17 \\
20 \\
22 \\
18\end{array}$ & $\begin{array}{l}-- \\
. \overline{90} \\
-- \\
--\end{array}$ \\
\hline $\begin{array}{l}85-05-03 \\
85-06-14 \\
85-07-24 \\
85-08-09 \\
85-08-07\end{array}$ & $\begin{array}{l}-- \\
.3 \\
.1 \\
.2 \\
.7\end{array}$ & $\begin{array}{l}644 \\
642 \\
646 \\
641\end{array}$ & $\begin{array}{l}3 \overline{340} \\
280 \\
280 \\
200\end{array}$ & $\begin{array}{l}-\overline{0} \\
410 \\
340 \\
340 \\
240\end{array}$ & $\begin{array}{l}-- \\
0 \\
0 \\
0 \\
0\end{array}$ & $\begin{array}{l}-- \\
41 \\
27 \\
34 \\
24\end{array}$ & $\begin{array}{r}\overline{-} \\
270 \\
\overline{-} \\
240 \\
200\end{array}$ & $\begin{array}{l}\overline{72} \\
-\overline{64} \\
61\end{array}$ & $\begin{array}{l}22^{--} \\
20^{--} \\
12\end{array}$ & $\begin{array}{l}16^{--} \\
15^{--} \\
13\end{array}$ & $16^{--}-$ & $\begin{array}{r}.- \\
.80 \\
-8 \\
.80 \\
.70\end{array}$ \\
\hline
\end{tabular}


Table 3.--Ground-water quality data, 1984 and 1985--Continued

\begin{tabular}{|c|c|c|c|c|c|c|c|c|c|c|c|c|}
\hline $\begin{array}{c}\text { DATE } \\
\text { OF } \\
\text { SAMPLE }\end{array}$ & $\begin{array}{l}\text { CHLO- } \\
\text { RIDE, } \\
\text { DIS- } \\
\text { SOLVED } \\
\text { (MG/L } \\
\text { AS CL) }\end{array}$ & $\begin{array}{l}\text { SULFATE, } \\
\text { DIS- } \\
\text { SOLVED } \\
\text { (MG/L } \\
\text { AS SO4) }\end{array}$ & $\begin{array}{l}\text { SILICA, } \\
\text { DIS- } \\
\text { SOLVED } \\
\text { (MG/L } \\
\text { AS } \\
\text { SIO2) }\end{array}$ & $\begin{array}{l}\text { CARBON, } \\
\text { ORGANIC, } \\
\text { DISSOLVED } \\
\text { (MG } / L \\
\text { AS C) }\end{array}$ & $\begin{array}{l}\text { METHY- } \\
\text { LENE } \\
\text { BLUE } \\
\text { ACTIVE } \\
\text { SUB- } \\
\text { STANCE } \\
\text { (MG/L) }\end{array}$ & $\begin{array}{c}\text { NITRO- } \\
\text { GEN, } \\
\text { NO2+NO3 } \\
\text { DIS- } \\
\text { SOLVED } \\
\text { (MG/L } \\
\text { AS N) }\end{array}$ & $\begin{array}{l}\text { NITRO- } \\
\text { GEN, } \\
\text { AMMONIA } \\
\text { DIS- } \\
\text { SOLVED } \\
\text { (MG/L } \\
\text { AS N) }\end{array}$ & $\begin{array}{c}\text { NITRO- } \\
\text { GEN,AM- } \\
\text { MONIA + } \\
\text { ORGANIC } \\
\text { DISSOLVED } \\
\text { (MG/L } \\
\text { AS N) }\end{array}$ & $\begin{array}{l}\text { NITRO- } \\
\text { GEN, } \\
\text { ORGANIC } \\
\text { DIS- } \\
\text { SOLVED } \\
\text { (MG/L } \\
\text { AS N) }\end{array}$ & $\begin{array}{l}\text { NITRO- } \\
\text { GEN, } \\
\text { AMMONIA, } \\
\text { DIS- } \\
\text { SOLVED } \\
\text { (MG/L } \\
\text { AS NH4) }\end{array}$ & $\begin{array}{l}\text { PHOS- } \\
\text { PHORUS, } \\
\text { DIS- } \\
\text { SOLVED } \\
\text { (MG / } \\
\text { AS P) }\end{array}$ & $\begin{array}{l}\text { IRON, } \\
\text { DIS- } \\
\text { SOLVED } \\
\text { (UG/L } \\
\text { AS FE) }\end{array}$ \\
\hline $\begin{array}{l}84-03-09 \\
84-04-11 \\
84-05-25 \\
84-10-10 \\
85-06-13\end{array}$ & $\begin{array}{l}-- \\
-- \\
10^{--}\end{array}$ & $\begin{array}{l}-- \\
29 \\
-- \\
31\end{array}$ & $\begin{array}{l}-- \\
\overline{--} \\
\overline{22}\end{array}$ & $\begin{array}{r}0.60 \\
.40 \\
.30 \\
-- \\
1.5\end{array}$ & $\begin{array}{l}-- \\
-- \\
-- \\
--\end{array}$ & $\begin{array}{l}0.28 \\
.47 \\
.35 \\
.16 \\
.54\end{array}$ & $\begin{array}{r}0.040 \\
.030 \\
<.010 \\
<.010 \\
.020\end{array}$ & $\begin{array}{r}--- \\
0.20 \\
.30 \\
<.20 \\
--\end{array}$ & $\begin{array}{r}0 . \overline{17} \\
-- \\
--\end{array}$ & $\begin{array}{r}0.05 \\
.04 \\
-- \\
.03\end{array}$ & $\begin{array}{r}-- \\
0.050 \\
.010 \\
--\end{array}$ & $\begin{array}{r}70 \\
9 \\
<10 \\
16 \\
<3\end{array}$ \\
\hline $\begin{array}{l}84-05-22 \\
84-07-18 \\
84-10-10 \\
84-01-31 \\
84-03-06\end{array}$ & $12^{--}$ & $\begin{array}{l}-- \\
-- \\
34 \\
--\end{array}$ & $\begin{array}{l}-- \\
-- \\
-- \\
--\end{array}$ & $\begin{array}{c}.60 \\
.80 \\
2.0 \\
-8 \\
.80\end{array}$ & $\begin{array}{l}-- \\
-- \\
-- \\
--\end{array}$ & $\begin{array}{r}7.9 \\
1.3 \\
1.1 \\
<1.6 \\
1.7\end{array}$ & $\begin{array}{l}1.10 \\
.510 \\
.340 \\
1.10\end{array}$ & $\begin{array}{l}2.4 \\
.80 \\
.80 \\
-- \\
--\end{array}$ & $\begin{array}{l}1.3 \\
.29 \\
.46 \\
-- \\
--\end{array}$ & $\begin{array}{l}1.4 \\
.66 \\
.44 \\
-.4\end{array}$ & $\begin{array}{r}.130 \\
.060 \\
.060 \\
-- \\
--\end{array}$ & $\begin{array}{r}20 \\
3 \\
6 \\
30 \\
30\end{array}$ \\
\hline $\begin{array}{l}84-04-13 \\
84-05-24 \\
84-07-26 \\
84-10-04 \\
84-05-23\end{array}$ & $\begin{array}{l}-- \\
=- \\
-- \\
--\end{array}$ & $\begin{array}{l}41 \\
-- \\
-- \\
--\end{array}$ & $\begin{array}{l}-- \\
-- \\
-- \\
--\end{array}$ & $\begin{array}{l}.60 \\
.80 \\
-7 \\
3.7 \\
.70\end{array}$ & $\begin{array}{c}0.07 \\
-- \\
-- \\
--\end{array}$ & $\begin{array}{l}6.2 \\
5.5 \\
6.5 \\
4.9 \\
8.0\end{array}$ & $\begin{array}{l}3.50 \\
1.60 \\
4.40 \\
3.50 \\
2.90\end{array}$ & $\begin{array}{l}3.5 \\
7.5 \\
6.3 \\
4.0 \\
4.1\end{array}$ & $\begin{array}{l}.00 \\
5.9 \\
1.9 \\
.50 \\
1.2\end{array}$ & $\begin{array}{l}4.5 \\
2.1 \\
5.7 \\
4.5 \\
3.7\end{array}$ & $\begin{array}{r}-- \\
.340 \\
.360 \\
.360 \\
.270\end{array}$ & $\begin{array}{r}8 \\
30 \\
-- \\
21 \\
<10\end{array}$ \\
\hline $\begin{array}{l}84-07-25 \\
84-10-10 \\
84-04-12 \\
84-05-23 \\
84-07-25\end{array}$ & $\begin{array}{l}-- \\
-- \\
-- \\
--\end{array}$ & $\begin{array}{l}-- \\
-- \\
36 \\
--\end{array}$ & $\begin{array}{l}-- \\
-- \\
-- \\
--\end{array}$ & $\begin{array}{l}2 .- \\
.90 \\
.80 \\
--\end{array}$ & $\begin{array}{l}-- \\
-- \\
-- \\
--\end{array}$ & $\begin{array}{l}9.5 \\
6.9 \\
3.8 \\
3.0 \\
2.1\end{array}$ & $\begin{array}{l}7.30 \\
4.80 \\
.070 \\
<.010 \\
.010\end{array}$ & $\begin{array}{l}8.6 \\
6.0 \\
.80 \\
1.0 \\
.50\end{array}$ & $\begin{array}{l}1.3 \\
1.2 \\
.73 \\
-4 \\
.49\end{array}$ & $\begin{array}{l}9.4 \\
6.2 \\
.09 \\
-- \\
.01\end{array}$ & $\begin{array}{r}.620 \\
.560 \\
- \\
.080 \\
--\end{array}$ & $\begin{array}{r}-- \\
4 \\
9 \\
<10 \\
--\end{array}$ \\
\hline $\begin{array}{l}84-10-11 \\
84-04-12 \\
84-05-24 \\
84-07-26 \\
84-10-11\end{array}$ & $\begin{array}{l}-- \\
-- \\
-- \\
--\end{array}$ & $\begin{array}{l}-- \\
35 \\
-- \\
--\end{array}$ & $\begin{array}{l}-- \\
-- \\
-- \\
--\end{array}$ & $\begin{array}{l}-- \\
.50 \\
.70 \\
-- \\
--\end{array}$ & $\begin{array}{l}-- \\
-- \\
-- \\
--\end{array}$ & $\begin{array}{l}2.5 \\
1.8 \\
1.1 \\
1.5 \\
1.5\end{array}$ & $\begin{array}{r}<.010 \\
.070 \\
<.010 \\
.020 \\
<.010\end{array}$ & $\begin{array}{r}.40 \\
.90 \\
1.0 \\
.30 \\
.20\end{array}$ & $\begin{array}{l}-\overline{-} \\
.83 \\
\overline{-28} \\
.-\end{array}$ & $\begin{array}{l}.09 \\
.09 \\
.03 \\
--\end{array}$ & $\begin{array}{r}.030 \\
-0 \\
.010 \\
-- \\
--\end{array}$ & $\begin{array}{r}<3 \\
9 \\
20 \\
-- \\
--\end{array}$ \\
\hline $\begin{array}{l}85-08-08 \\
85-08-08 \\
84-02-02 \\
84-02-28 \\
84-04-13\end{array}$ & $\begin{array}{l}9 \cdot 5 \\
14 \\
16 \\
19\end{array}$ & $\begin{array}{l}--- \\
31 \\
27 \\
21 \\
15\end{array}$ & $\begin{array}{l}-- \\
17 \\
24 \\
24 \\
26\end{array}$ & $\begin{array}{l}1.0 \\
1.0 \\
2.0 \\
1.6\end{array}$ & $\begin{array}{c}-- \\
-- \\
-- \\
-01\end{array}$ & $\begin{array}{r}-- \\
.22 \\
.48 \\
<.10 \\
<.10\end{array}$ & $\begin{array}{l}.030 \\
.03 \\
1.70 \\
2.50\end{array}$ & $\begin{array}{l}.- \\
.20 \\
-\overline{-} \\
3.0\end{array}$ & $\begin{array}{l}.- \\
.17 \\
\overline{--} \\
.50\end{array}$ & $\begin{array}{c}. \overline{.04} \\
2 . \overline{2} \\
3.2\end{array}$ & $\begin{array}{l}-- \\
-- \\
-- \\
--\end{array}$ & $\begin{array}{r}15 \\
31 \\
3600 \\
3300 \\
4900\end{array}$ \\
\hline $\begin{array}{l}84-05-25 \\
84-07-27 \\
84-10-04 \\
85-04-05 \\
85-04-18\end{array}$ & $11=$ & $\begin{array}{l}-- \\
-- \\
27 \\
--\end{array}$ & $\begin{array}{l}-- \\
-- \\
27 \\
--\end{array}$ & $\begin{array}{r}1.8 \\
1.8 \\
2.6 \\
-- \\
--\end{array}$ & $\begin{array}{l}-- \\
-- \\
-- \\
--\end{array}$ & $\begin{array}{l}<.10 \\
<.10 \\
<.10 \\
<.10\end{array}$ & $\begin{array}{r}2.60 \\
2.70 \\
2.30 \\
2.10\end{array}$ & $\begin{array}{c}3.7 \\
2.9 \\
2.1 \\
-- \\
2.7\end{array}$ & $\begin{array}{r}1.1 \\
.20 \\
-.20 \\
-60\end{array}$ & $\begin{array}{c}3.3 \\
3.5 \\
3.0 \\
2.7\end{array}$ & $\begin{array}{r}.120 \\
.170 \\
.200 \\
-- \\
--\end{array}$ & $\begin{array}{r}3100 \\
<2300 \\
2700 \\
6300 \\
4300\end{array}$ \\
\hline $\begin{array}{l}85-05-03 \\
85-06-14 \\
85-07-24 \\
85-08-09 \\
85-08-07\end{array}$ & $\begin{array}{c}14^{--} \\
16^{--} \\
8.0\end{array}$ & $\begin{array}{l}-- \\
12 \\
--- \\
31\end{array}$ & $\begin{array}{l}-- \\
28 \\
-- \\
27 \\
15\end{array}$ & $\begin{array}{l}2 .-- \\
2.9 \\
2.5 \\
2.8\end{array}$ & $\begin{array}{l}-- \\
-- \\
-- \\
--\end{array}$ & $\begin{array}{r}-- \\
<.10 \\
<.10 \\
<.10 \\
.38\end{array}$ & $\begin{array}{l}--- \\
2.20 \\
1.70 \\
2.70 \\
.080\end{array}$ & $\begin{array}{l}-- \\
2.8 \\
2.7 \\
.30\end{array}$ & $\begin{array}{l}-- \\
1.1 \\
.00 \\
.22\end{array}$ & $\begin{array}{l}-- \\
2.8 \\
2.2 \\
3.5 \\
.10\end{array}$ & $\begin{array}{l}-- \\
-- \\
-- \\
--\end{array}$ & $\begin{array}{r}-- \\
4200 \\
4900 \\
5300 \\
390\end{array}$ \\
\hline
\end{tabular}


Table 3.--Ground-water quality data, 1984 and 1985--Continued

\begin{tabular}{|c|c|c|c|c|}
\hline $\begin{array}{c}\text { DATE } \\
\text { OF } \\
\text { SAMPLE }\end{array}$ & $\begin{array}{l}\text { IRON, } \\
\text { SUS- } \\
\text { PENDED } \\
\text { RECOV- } \\
\text { ERABLE } \\
\text { (UG/L } \\
\text { AS FE) }\end{array}$ & $\begin{array}{l}\text { I RON, } \\
\text { TOTAL } \\
\text { RECOV- } \\
\text { ERABLE } \\
\text { (UG/L } \\
\text { AS FE) }\end{array}$ & $\begin{array}{l}\text { MANGA- } \\
\text { NESE, } \\
\text { DIS- } \\
\text { SOLVED } \\
\text { (UG/L } \\
\text { AS MN) }\end{array}$ & $\begin{array}{l}\text { ZINC, } \\
\text { DIS- } \\
\text { SOLVED } \\
\text { (UG/L } \\
\text { AS ZN) }\end{array}$ \\
\hline
\end{tabular}

\begin{tabular}{|c|c|c|c|c|}
\hline $\begin{array}{l}84-03-09 \\
84-04-11 \\
84-05-25 \\
84-10-10 \\
85-06-13\end{array}$ & $\begin{array}{l}-- \\
90 \\
-- \\
-- \\
--\end{array}$ & $\begin{array}{r}-- \\
100 \\
10 \\
-- \\
--\end{array}$ & $\begin{array}{l}50 \\
19 \\
40 \\
78 \\
21\end{array}$ & $\begin{array}{l}-- \\
83 \\
50 \\
51 \\
35\end{array}$ \\
\hline $\begin{array}{l}84-05-22 \\
84-07-18 \\
84-10-10 \\
84-01-31 \\
84-03-06\end{array}$ & $\begin{array}{l}-- \\
-- \\
-- \\
-- \\
--\end{array}$ & $\begin{array}{r}40 \\
580 \\
-- \\
-- \\
--\end{array}$ & $\begin{array}{l}10 \\
12 \\
18 \\
28 \\
70\end{array}$ & $\begin{array}{r}130 \\
140 \\
290 \\
-- \\
--\end{array}$ \\
\hline $\begin{array}{l}84-04-13 \\
84-05-24 \\
84-07-26 \\
84-10-04 \\
84-05-23\end{array}$ & $\begin{array}{l}30 \\
-- \\
-- \\
-- \\
--\end{array}$ & $\begin{array}{l}40 \\
20 \\
-- \\
-- \\
20\end{array}$ & $\begin{array}{r}7 \\
20 \\
-- \\
29 \\
<10\end{array}$ & $\begin{array}{r}48 \\
100 \\
-- \\
45 \\
70\end{array}$ \\
\hline $\begin{array}{l}84-07-25 \\
84-10-10 \\
84-04-12 \\
84-05-23 \\
84-07-25\end{array}$ & $\begin{array}{l}-- \\
-- \\
20 \\
-- \\
--\end{array}$ & $\begin{array}{l}-- \\
-- \\
30 \\
30 \\
--\end{array}$ & $\begin{array}{r}- \\
6 \\
1 \\
<10 \\
--\end{array}$ & $\begin{array}{r}70 \\
51 \\
140 \\
40 \\
60\end{array}$ \\
\hline $\begin{array}{l}84-10-11 \\
84-04-12 \\
84-05-24 \\
84-07-26 \\
84-10-11\end{array}$ & $\begin{array}{r}-- \\
120 \\
-- \\
-- \\
--\end{array}$ & $\begin{array}{l}-- \\
130 \\
130 \\
-- \\
--\end{array}$ & $\begin{array}{r}<1 \\
2 \\
<10 \\
-- \\
--\end{array}$ & $\begin{array}{r}56 \\
160 \\
100 \\
90 \\
60\end{array}$ \\
\hline $\begin{array}{l}85-08-08 \\
85-08-08 \\
84-02-02 \\
84-02-28 \\
84-04-13\end{array}$ & $\begin{array}{l}-- \\
-- \\
-- \\
-- \\
100\end{array}$ & $\begin{array}{c}-- \\
-- \\
-- \\
\overline{--}\end{array}$ & $\begin{array}{r}140 \\
20 \\
2500 \\
2800 \\
3700\end{array}$ & $\begin{array}{l}26 \\
18 \\
-- \\
-- \\
27\end{array}$ \\
\hline $\begin{array}{l}84-05-25 \\
84-07-27 \\
84-10-04 \\
85-04-05 \\
85-04-18\end{array}$ & $\begin{array}{l}-- \\
-- \\
-- \\
-- \\
--\end{array}$ & $\begin{array}{c}4300 \\
-- \\
-- \\
-- \\
--\end{array}$ & $\begin{array}{l}2900 \\
2300 \\
2300 \\
5100 \\
4900\end{array}$ & $\begin{array}{r}30 \\
14 \\
19 \\
120 \\
10\end{array}$ \\
\hline $\begin{array}{l}85-05-03 \\
85-06-14 \\
85-07-24 \\
85-08-09 \\
85-08-07\end{array}$ & $\begin{array}{l}-- \\
-- \\
-- \\
--\end{array}$ & $\begin{array}{l}-- \\
-- \\
-- \\
-- \\
--\end{array}$ & $\begin{array}{r}4100 \\
3400 \\
3500 \\
280\end{array}$ & $\begin{array}{r}-- \\
20 \\
92 \\
33 \\
120\end{array}$ \\
\hline
\end{tabular}


Table 3.--Ground-water quality data, 1984 and 1985--Continued

\begin{tabular}{|c|c|c|c|c|c|c|c|c|c|c|c|}
\hline $\begin{array}{l}\text { WELL } \\
\text { NUM- } \\
\text { BER }\end{array}$ & $\begin{array}{l}\text { WELL } \\
\text { LOCA- } \\
\text { TION }\end{array}$ & $\begin{array}{c}\text { DATE } \\
\text { OF } \\
\text { SAMPLE }\end{array}$ & TIME & $\begin{array}{l}\text { SPE- } \\
\text { CIFIC } \\
\text { CONDUC- } \\
\text { TANCE* } \\
\text { (UMHOS) }\end{array}$ & $\begin{array}{l}\text { SPE- } \\
\text { CIFIC } \\
\text { CONDUC- } \\
\text { TANCE, } \\
\text { LAB } \\
\text { (UMHOS) }\end{array}$ & $\begin{array}{l}\text { SOLIDS, } \\
\text { SUM OF } \\
\text { CONSTI- } \\
\text { TUENTS, } \\
\text { DIS- } \\
\text { SOLVED } \\
\text { (MG/L) }\end{array}$ & $\begin{array}{l}\text { SOLIDS, } \\
\text { RESIDUE } \\
\text { AT } 180 \\
\text { DEG. C, } \\
\text { DIS- } \\
\text { SOLVED } \\
(M G / L)\end{array}$ & $\begin{array}{l}\text { SOLIDS/ } \\
\text { SPE- } \\
\text { CIFIC } \\
\text { CONDUC- } \\
\text { TANCE, } \\
\text { RATIO }\end{array}$ & $\begin{array}{l}\text { CATION/ } \\
\text { ANION, } \\
\text { PERCENT } \\
\text { DIFFER- } \\
\text { ENCE }\end{array}$ & $\begin{array}{l}\text { TEMPER- } \\
\text { ATURE* } \\
\text { (DEG. C) }\end{array}$ & $\begin{array}{c}\text { PH* } \\
\text { (STAN- } \\
\text { DARD } \\
\text { UNITS) }\end{array}$ \\
\hline $\begin{array}{l}\text { M1. } \\
137 \text {. }\end{array}$ & $\begin{array}{l}2 S-35 E-34 C B C B 1 \\
3 S-35 E-5 B A D C 1\end{array}$ & $\begin{array}{l}85-08-08 \\
84-04-11 \\
84-05-31 \\
84-07-26 \\
84-10-12\end{array}$ & $\begin{array}{l}1530 \\
1745 \\
1500 \\
1515 \\
1440\end{array}$ & $\begin{array}{l}381 \\
372 \\
267 \\
294 \\
315\end{array}$ & $\begin{array}{r}385 \\
-- \\
270 \\
295 \\
320\end{array}$ & $\begin{array}{r}240 \\
-- \\
\overline{-} \\
190\end{array}$ & $\begin{array}{r}220 \\
-- \\
\overline{172}\end{array}$ & $\begin{array}{r}0.58 \\
=- \\
\overline{-5} \\
.55\end{array}$ & $\begin{array}{r}-1.27 \\
=- \\
-\overline{-} \\
-1.51\end{array}$ & $\begin{array}{r}13.5 \\
5.5 \\
10.0 \\
17.0 \\
12.5\end{array}$ & $\begin{array}{l}7.3 \\
7.9 \\
7.9 \\
7.6 \\
7.7\end{array}$ \\
\hline $\begin{array}{l}138 . \\
139 A .\end{array}$ & $\begin{array}{l}3 \mathrm{~S}-35 \mathrm{E}-5 \mathrm{BCBCl} \\
3 \mathrm{~S}-35 \mathrm{E}-5 \mathrm{BCDD} 1\end{array}$ & $\begin{array}{l}84-05-31 \\
84-10-11 \\
84-03-09 \\
84-04-12 \\
84-05-31\end{array}$ & $\begin{array}{l}1645 \\
1130 \\
1500 \\
1645 \\
1545\end{array}$ & $\begin{array}{l}362 \\
387 \\
425 \\
527 \\
602\end{array}$ & $\begin{array}{l}361 \\
398 \\
437 \\
521 \\
589\end{array}$ & $\begin{array}{r}-- \\
270 \\
320 \\
--\end{array}$ & $\begin{array}{r}-- \\
266 \\
316 \\
--\end{array}$ & $\begin{array}{l}-- \\
.- \\
.63 \\
.60 \\
--\end{array}$ & $\begin{array}{r}-- \\
-1.50 \\
-2.37 \\
--\end{array}$ & $\begin{array}{r}9.5 \\
9.5 \\
11.0 \\
8.5 \\
9.0\end{array}$ & $\begin{array}{l}7.6 \\
7.4 \\
7.3 \\
6.9 \\
7.1\end{array}$ \\
\hline 140 . & $3 S-35 E-5 C B C B 1$ & $\begin{array}{l}84-07-26 \\
84-10-12 \\
84-02-29 \\
84-04-10 \\
84-05-22\end{array}$ & $\begin{array}{l}1645 \\
1600 \\
1445 \\
1520 \\
0945\end{array}$ & $\begin{array}{l}518 \\
458 \\
395 \\
395 \\
447\end{array}$ & $\begin{array}{l}510 \\
464 \\
403 \\
403 \\
440\end{array}$ & $\begin{array}{r}-\overline{-} \\
240 \\
240 \\
--\end{array}$ & $\begin{array}{r}-- \\
236 \\
241 \\
--\end{array}$ & $\begin{array}{l}. \overline{61} \\
.60 \\
.61 \\
--\end{array}$ & $\begin{array}{r}-1.51 \\
-3.29 \\
-1.31 \\
--\end{array}$ & $\begin{array}{r}12.5 \\
13.0 \\
9.0 \\
9.0 \\
9.0\end{array}$ & $\begin{array}{l}7.0 \\
7.1 \\
7.6 \\
7.6 \\
7.5\end{array}$ \\
\hline 147. & $3 S-35 E-11 B C C B 1$ & $\begin{array}{l}84-07-19 \\
84-10-11 \\
84-04-12 \\
84-05-31\end{array}$ & $\begin{array}{l}1310 \\
0920 \\
1430 \\
1915\end{array}$ & $\begin{array}{l}454 \\
431 \\
599 \\
613\end{array}$ & $\begin{array}{r}470 \\
434 \\
562 \\
--\end{array}$ & $\begin{array}{c}-- \\
270 \\
--\end{array}$ & $\begin{array}{r}\overline{-} \\
331 \\
--\end{array}$ & $\begin{array}{l}.-- \\
.62 \\
.55 \\
--\end{array}$ & $\begin{array}{r}-- \\
-6.58 \\
--\end{array}$ & $\begin{array}{r}10.5 \\
9.0 \\
15.0 \\
15.5\end{array}$ & $\begin{array}{l}7.4 \\
7.5 \\
7.2 \\
7.3\end{array}$ \\
\hline
\end{tabular}


Table 3.--Ground-water quality data, 1984 and 1985--Continued

\begin{tabular}{|c|c|c|c|c|c|c|c|c|c|c|c|c|}
\hline $\begin{array}{c}\text { DATE } \\
\text { OF } \\
\text { SAMPLE }\end{array}$ & $\begin{array}{c}\text { OXYGEN*, } \\
\text { DIS- } \\
\text { SOLVED } \\
(M G / L)\end{array}$ & $\begin{array}{l}\text { BARO- } \\
\text { METRIC } \\
\text { PRES- } \\
\text { SURE* } \\
\text { (MM } \\
\text { OF HG) }\end{array}$ & $\begin{array}{l}\text { ALKA- } \\
\text { LINITY* } \\
\text { (MG/L } \\
\text { AS } \\
\text { CACO3) }\end{array}$ & $\begin{array}{l}\text { BICAR- } \\
\text { BONATE* } \\
\text { FET-FLD } \\
\text { (MG/L AS } \\
\text { HCO3) }\end{array}$ & $\begin{array}{c}\text { CAR- } \\
\text { BONATE* } \\
\text { FET-FLD } \\
\text { (MG/L } \\
\text { AS CO } 3 \text { ) }\end{array}$ & $\begin{array}{c}\text { CARBON } \\
\text { DIOXIDE, } \\
\text { DIS- } \\
\text { SOLVED } \\
\text { (MG/L } \\
\text { AS CO2) }\end{array}$ & $\begin{array}{l}\text { HARD- } \\
\text { NESS } \\
\text { (MG/L } \\
\text { AS } \\
\text { CACO3) }\end{array}$ & $\begin{array}{c}\text { CALCIUM, } \\
\text { DIS- } \\
\text { SOLVED } \\
\text { (MG/L } \\
\text { AS CA) }\end{array}$ & $\begin{array}{l}\text { MAGNE- } \\
\text { SIUM, } \\
\text { DIS- } \\
\text { SOLVED } \\
\text { (MG/L } \\
\text { AS MG) }\end{array}$ & $\begin{array}{l}\text { SODIUM, } \\
\text { DIS- } \\
\text { SOLVED } \\
\text { (MG/L } \\
\text { AS NA) }\end{array}$ & $\begin{array}{l}\text { POTAS- } \\
\text { SIUM, } \\
\text { DIS- } \\
\text { SOLVED } \\
\text { (MG/L } \\
\text { AS K) }\end{array}$ & $\begin{array}{l}\text { FLUO- } \\
\text { RIDE, } \\
\text { DIS- } \\
\text { SOLVED } \\
\text { (MG/L } \\
\text { AS F) }\end{array}$ \\
\hline $\begin{array}{l}85-08-08 \\
84-04-11 \\
84-05-31 \\
84-07-26 \\
84-10-12\end{array}$ & $\begin{array}{l}0.5 \\
5.0 \\
7.7 \\
2.8 \\
5.0\end{array}$ & $\begin{array}{r}641 \\
-\overline{641} \\
645 \\
644\end{array}$ & $\begin{array}{l}180 \\
140 \\
110 \\
130 \\
120\end{array}$ & $\begin{array}{l}220 \\
180 \\
140 \\
160 \\
150\end{array}$ & $\begin{array}{l}0 \\
0 \\
0 \\
0 \\
0\end{array}$ & $\begin{array}{r}18 \\
3.6 \\
2.8 \\
6.4 \\
4.8\end{array}$ & $\begin{array}{r}180 \\
-- \\
-- \\
140\end{array}$ & $\begin{array}{l}55 \\
-- \\
-- \\
--\end{array}$ & $\begin{array}{l}11 \\
=- \\
9.1\end{array}$ & 11 & $\begin{array}{r}2.6 \\
-\frac{5}{1.9} \\
1.7\end{array}$ & $\begin{array}{r}0.60 \\
-- \\
-- \\
.70\end{array}$ \\
\hline $\begin{array}{l}84-05-31 \\
84-10-11 \\
84-03-09 \\
84-04-12 \\
84-05-31\end{array}$ & $\begin{array}{r}4.2 \\
.8 \\
.6 \\
1.1 \\
2.0\end{array}$ & $\begin{array}{l}640 \\
635 \\
648 \\
642 \\
640\end{array}$ & $\begin{array}{l}160 \\
180 \\
160 \\
210 \\
240\end{array}$ & $\begin{array}{l}190 \\
220 \\
200 \\
260 \\
290\end{array}$ & $\begin{array}{l}0 \\
0 \\
0 \\
0 \\
0\end{array}$ & $\begin{array}{l}7.6 \\
14 \\
16 \\
52 \\
37\end{array}$ & $\begin{array}{r}-- \\
190 \\
240 \\
--\end{array}$ & $\begin{array}{l}-- \\
-\overline{56} \\
73 \\
--\end{array}$ & $\begin{array}{l}11^{--} \\
14\end{array}$ & $\begin{array}{l}11^{--} \\
12\end{array}$ & $\begin{array}{l}2.1 \\
2.3 \\
5.5 \\
4.8 \\
4.5\end{array}$ & $\begin{array}{l}-- \\
-\overline{70} \\
.70 \\
.-\end{array}$ \\
\hline $\begin{array}{l}84-07-26 \\
84-10-12 \\
84-02-29 \\
84-04-10 \\
84-05-22\end{array}$ & $\begin{array}{r}1.3 \\
.1 \\
-- \\
.4 \\
.3\end{array}$ & $\begin{array}{r}643 \\
644 \\
-- \\
636 \\
642\end{array}$ & $\begin{array}{l}240 \\
210 \\
170 \\
160 \\
190\end{array}$ & $\begin{array}{l}290 \\
260 \\
210 \\
200 \\
230\end{array}$ & $\begin{array}{l}0 \\
0 \\
0 \\
0 \\
0\end{array}$ & $\begin{array}{l}46 \\
33 \\
8.4 \\
8.0 \\
12\end{array}$ & $\begin{array}{l}-- \\
210 \\
180 \\
180 \\
--\end{array}$ & $\begin{array}{l}-- \\
67 \\
50 \\
50 \\
--\end{array}$ & $\begin{array}{l}11^{--} \\
13 \\
13 \\
--\end{array}$ & $\begin{array}{l}11^{--} \\
11 \\
11\end{array}$ & $\begin{array}{l}-- \\
5.4 \\
2.4 \\
2.2 \\
2.4\end{array}$ & $\begin{array}{l}-- \\
.70 \\
.80 \\
.80 \\
--\end{array}$ \\
\hline $\begin{array}{l}84-07-19 \\
84-10-11 \\
84-04-12 \\
84-05-31\end{array}$ & $\begin{array}{l}.4 \\
.1 \\
.7 \\
.4\end{array}$ & $\begin{array}{l}646 \\
636 \\
641 \\
639\end{array}$ & $\begin{array}{l}220 \\
200 \\
290 \\
300\end{array}$ & $\begin{array}{l}260 \\
240 \\
350 \\
360\end{array}$ & $\begin{array}{l}0 \\
0 \\
0 \\
0\end{array}$ & $\begin{array}{l}16 \\
12 \\
35 \\
29\end{array}$ & $\begin{array}{c}-- \\
190 \\
--\end{array}$ & $\begin{array}{l}-- \\
56 \\
--\end{array}$ & $13^{--}$ & $11^{--}$ & $\begin{array}{l}2.5 \\
2.4 \\
3.4 \\
3.6\end{array}$ & $\begin{array}{l}-\overline{70} \\
-- \\
--\end{array}$ \\
\hline
\end{tabular}


Table 3.--Ground-water quality data, 1984 and 1985--Continued

\begin{tabular}{|c|c|c|c|c|c|c|c|c|c|c|c|c|}
\hline $\begin{array}{c}\text { DATE } \\
\text { OF } \\
\text { SAMPLE }\end{array}$ & $\begin{array}{l}\text { CHLO- } \\
\text { RIDE, } \\
\text { DIS- } \\
\text { SOLVED } \\
\text { (MG/L } \\
\text { AS CL) }\end{array}$ & $\begin{array}{l}\text { SULFATE, } \\
\text { DIS- } \\
\text { SOLVED } \\
\text { (MG/L } \\
\text { AS SO4) }\end{array}$ & $\begin{array}{l}\text { SILICA, } \\
\text { DIS- } \\
\text { SOLVED } \\
\text { (MG/L } \\
\text { AS } \\
\text { SIO2) }\end{array}$ & $\begin{array}{l}\text { CARBON, } \\
\text { ORGANIC, } \\
\text { DISSOLVED } \\
\text { (MG / L } \\
\text { AS C) }\end{array}$ & $\begin{array}{l}\text { METHY- } \\
\text { LENE } \\
\text { BLUE } \\
\text { ACT IVE } \\
\text { SUB- } \\
\text { STANCE } \\
\text { (MG/L) }\end{array}$ & $\begin{array}{c}\text { NITRO- } \\
\text { GEN } \\
\text { NO2+NO3 } \\
\text { DIS- } \\
\text { SOLVED } \\
\text { (MG/L } \\
\text { AS N) }\end{array}$ & $\begin{array}{l}\text { NITRO- } \\
\text { GEN, } \\
\text { AMMONIA } \\
\text { DIS- } \\
\text { SOLVED } \\
\text { (MG/L } \\
\text { AS N) }\end{array}$ & $\begin{array}{l}\text { NITRO- } \\
\text { GEN,AM- } \\
\text { MONIA + } \\
\text { ORGANIC } \\
\text { DISSOLVED } \\
\text { (MG/L } \\
\text { AS N) }\end{array}$ & $\begin{array}{c}\text { NITRO- } \\
\text { GEN, } \\
\text { ORGANIC } \\
\text { DIS- } \\
\text { SOLVED } \\
\text { (MG/L } \\
\text { AS N) }\end{array}$ & $\begin{array}{c}\text { NITRO- } \\
\text { GEN, } \\
\text { AMMONIA, } \\
\text { DIS- } \\
\text { SOLVED } \\
\text { (MG/L } \\
\text { AS NH4) }\end{array}$ & $\begin{array}{l}\text { PHOS- } \\
\text { PHORUS, } \\
\text { DIS- } \\
\text { SOLVED } \\
\text { (MG/L } \\
\text { AS P) }\end{array}$ & $\begin{array}{l}\text { I RON, } \\
\text { DIS- } \\
\text { SOLVED } \\
\text { (UG/L } \\
\text { AS FE) }\end{array}$ \\
\hline $\begin{array}{l}85-08-08 \\
84-04-11 \\
84-05-31 \\
84-07-26 \\
84-10-12\end{array}$ & $\begin{array}{c}7.1 \\
-- \\
-- \\
-- \\
8.7\end{array}$ & $\begin{array}{l}23 \\
-- \\
-- \\
35\end{array}$ & $\begin{array}{l}18 \\
-- \\
-- \\
-- \\
13\end{array}$ & $\begin{array}{l}1.7 \\
-.- \\
1.2 \\
.90 \\
2.3\end{array}$ & $\begin{array}{l}-- \\
-- \\
-- \\
--\end{array}$ & $\begin{array}{r}<0.10 \\
-. \\
.23 \\
.25 \\
.16\end{array}$ & $\begin{array}{r}0.060 \\
<-0 \\
.010 \\
.050 \\
.020\end{array}$ & $\begin{array}{r}0.20 \\
-\overline{30} \\
<.20 \\
.30\end{array}$ & $\begin{array}{c}0.14 \\
-- \\
-- \\
.28\end{array}$ & $\begin{array}{c}0.08 \\
-- \\
-- \\
.06 \\
.03\end{array}$ & $\begin{array}{r}-\overline{-} \\
0.010 \\
.020\end{array}$ & $\begin{array}{c}280 \\
-- \\
36 \\
--\end{array}$ \\
\hline $\begin{array}{l}84-05-31 \\
84-10-11 \\
84-03-09 \\
84-04-12 \\
84-05-31\end{array}$ & $\begin{array}{l}-- \\
11^{--} \\
--\end{array}$ & $\begin{array}{l}-- \\
-- \\
43 \\
49 \\
--\end{array}$ & $\begin{array}{l}-- \\
-- \\
22 \\
19 \\
--\end{array}$ & $\begin{array}{l}1.0 \\
-- \\
1.1 \\
.70 \\
1.6\end{array}$ & $\frac{--}{--}$ & $\begin{array}{r}.87 \\
. .42 \\
. .10 \\
11.92\end{array}$ & $\begin{array}{r}<.010 \\
<.010 \\
.190 \\
.070 \\
<.010\end{array}$ & $\begin{array}{r}.50 \\
<.20 \\
-.5 \\
.30 \\
1.2\end{array}$ & $\begin{array}{l}-- \\
-- \\
-- \\
.23 \\
--\end{array}$ & $\begin{array}{l}-- \\
-- \\
.24 \\
.09 \\
--\end{array}$ & $\begin{array}{r}.010 \\
- \\
0 \\
.010\end{array}$ & $\begin{array}{r}7 \\
-\frac{7}{2000} \\
410 \\
180\end{array}$ \\
\hline $\begin{array}{l}84-07-26 \\
84-10-12 \\
84-02-29 \\
84-04-10\end{array}$ & $\begin{array}{r}9.0 \\
9.8 \\
10\end{array}$ & $\begin{array}{l}-- \\
26 \\
32 \\
33\end{array}$ & $\begin{array}{l}-- \\
19 \\
19 \\
20\end{array}$ & $\begin{array}{r}1.5 \\
2.1 \\
.90 \\
.70\end{array}$ & <.- & $\begin{array}{l}2.4 \\
<.10 \\
<.10 \\
<.10\end{array}$ & $\begin{array}{l}.030 \\
.050 \\
.070 \\
.120\end{array}$ & $\begin{array}{r}.50 \\
.20 \\
-- \\
<.20\end{array}$ & $\begin{array}{r}.47 \\
.15 \\
-- \\
--\end{array}$ & $\begin{array}{l}.04 \\
.06 \\
.09 \\
.15\end{array}$ & $<.010$ & $\begin{array}{l}110 \\
250 \\
370 \\
550\end{array}$ \\
\hline $84-05-22$ & -- & -- & -- & -- & -- & -- & -- & -- & -- & -- & -- & $<1200$ \\
\hline $\begin{array}{l}84-07-19 \\
84-10-11 \\
84-04-12 \\
84-05-31\end{array}$ & $\begin{array}{c}-- \\
-- \\
--\end{array}$ & $\begin{array}{l}-- \\
38 \\
22 \\
--\end{array}$ & $\begin{array}{l}21 \\
--\end{array}$ & $\begin{array}{l}1.1 \\
2.6 \\
.80 \\
.70\end{array}$ & $\begin{array}{c}-- \\
-01 \\
--\end{array}$ & $\begin{array}{l}<.10 \\
<.10 \\
.72 \\
1.5\end{array}$ & $\begin{array}{r}.070 \\
.030 \\
.040 \\
<.010\end{array}$ & $\begin{array}{r}<.20 \\
.20 \\
.40 \\
.50\end{array}$ & $\begin{array}{l}--17 \\
.36 \\
--\end{array}$ & $\begin{array}{l}.09 \\
.04 \\
.05 \\
--\end{array}$ & $\begin{array}{r}.010 \\
.010 \\
.010\end{array}$ & $\begin{array}{r}1300 \\
1300 \\
43 \\
13\end{array}$ \\
\hline
\end{tabular}


Table 3.--Ground-water quality data, 1984 and $1985--$ Continued

\begin{tabular}{|c|c|c|c|c|}
\hline $\begin{array}{c}\text { DATE } \\
\text { OF } \\
\text { SAMPLE }\end{array}$ & $\begin{array}{l}\text { IRON, } \\
\text { SUS- } \\
\text { PENDED } \\
\text { RECOV- } \\
\text { ERABLE } \\
\text { (UG/L } \\
\text { AS FE) }\end{array}$ & $\begin{array}{l}\text { I RON, } \\
\text { TOTAL } \\
\text { RECOV- } \\
\text { ERABLE } \\
\text { (UG/L } \\
\text { AS FE) }\end{array}$ & $\begin{array}{l}\text { MANGA- } \\
\text { NESE, } \\
\text { DIS- } \\
\text { SOLVED } \\
\text { (UG/L } \\
\text { AS MN) }\end{array}$ & $\begin{array}{l}\text { Z INC, } \\
\text { DIS- } \\
\text { SOLVED } \\
\text { (UG/L } \\
\text { AS ZN) }\end{array}$ \\
\hline $\begin{array}{l}85-08-08 \\
84-04-11 \\
84-05-31 \\
84-07-26 \\
84-10-12\end{array}$ & $\begin{array}{l}-- \\
-- \\
-- \\
--\end{array}$ & $\begin{array}{l}-- \\
-- \\
20 \\
-- \\
--\end{array}$ & $\begin{array}{c}210 \\
-\overline{1} \\
-- \\
14\end{array}$ & $\begin{array}{l}49 \\
-2 \\
45 \\
-- \\
51\end{array}$ \\
\hline $\begin{array}{l}84-05-31 \\
84-10-11 \\
84-03-09 \\
84-04-12 \\
84-05-31\end{array}$ & $\begin{array}{l}-- \\
-- \\
-- \\
--\end{array}$ & $\begin{array}{r}100 \\
-- \\
450 \\
290\end{array}$ & $\begin{array}{r}4 \\
-- \\
5100 \\
2500 \\
44\end{array}$ & $\begin{array}{r}30 \\
10 \\
-0 \\
61 \\
100\end{array}$ \\
\hline $\begin{array}{l}84-07-26 \\
84-10-12 \\
84-02-29 \\
84-04-10 \\
84-05-22\end{array}$ & $\begin{array}{l}-- \\
-- \\
--\end{array}$ & $\begin{array}{r}-- \\
-- \\
590 \\
1300\end{array}$ & $\begin{array}{l}540 \\
2100 \\
1200 \\
1400 \\
3100\end{array}$ & $\begin{array}{l}-- \\
32 \\
-\overline{71} \\
60\end{array}$ \\
\hline $\begin{array}{l}84-07-19 \\
84-10-11 \\
84-04-12 \\
84-05-31\end{array}$ & $\begin{array}{c}-- \\
170 \\
--\end{array}$ & $\begin{array}{r}1500 \\
-- \\
210 \\
20\end{array}$ & $\begin{array}{r}3800 \\
3300 \\
3 \\
9\end{array}$ & $\begin{array}{l}40 \\
69 \\
60 \\
68\end{array}$ \\
\hline
\end{tabular}




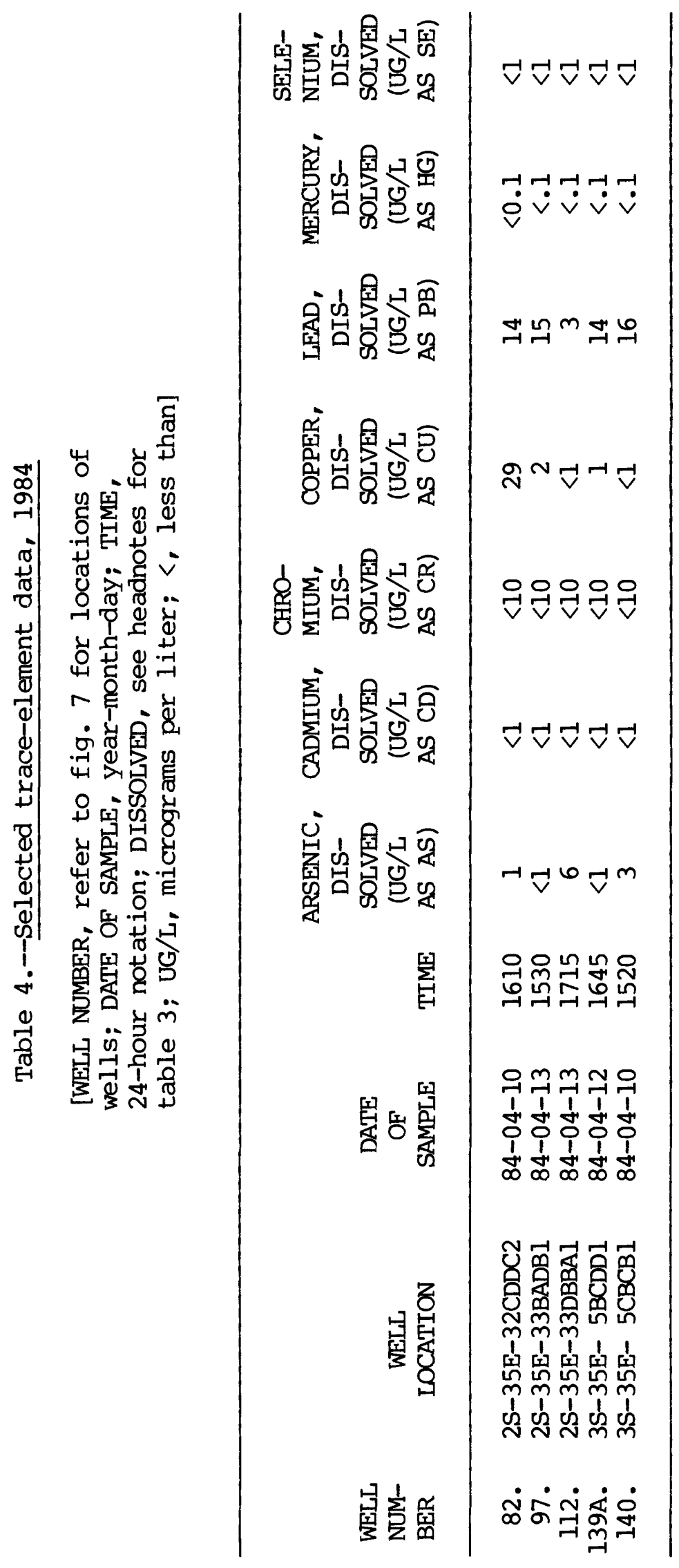


Table 5.--Statistical summary of selected water-quality data, 1984 and 1985

1*, onsite analysis; *, calculated value (see headnotes for table 3 ): mg/L, milligrams per 1 iter; $\mu \mathrm{g} / \mathrm{L}, \mathrm{microg} r \mathrm{~ms}$ per IIter; <, less thanj

\begin{tabular}{|c|c|c|c|c|c|c|c|}
\hline Water-quality constituent & $\begin{array}{c}\text { Data } \\
\text { population }\end{array}$ & $\begin{array}{l}90 \\
\text { percent }\end{array}$ & $\begin{array}{l}75 \\
\text { percent }\end{array}$ & $\begin{array}{l}\text { Median } \\
(50) \\
\text { percent }\end{array}$ & Mean & $\frac{\text { Range of }}{\text { Minimum }}$ & $\frac{\text { values }}{\text { Maximum }}$ \\
\hline *Specific conductance $(\mu \mathrm{mho} / \mathrm{cm})$ & 218 & 652 & 543 & 485 & 506 & 267 & 1,450 \\
\hline Specific conductance, lab $(\mu \mathrm{mho} / \mathrm{cm})$ & 185 & 646 & 520 & 478 & 482 & 270 & 1,060 \\
\hline * Solids, sum of constituents, dissolved (mg/L) & 73 & 362 & 320 & 290 & 290 & 190 & 520 \\
\hline Solids, residue at $180^{\circ} \mathrm{C}$, dissolved $(\mathrm{mg} / \mathrm{L})$ & 92 & 320 & 306 & 277 & 278 & 172 & 482 \\
\hline - Temperature $\left({ }^{\circ} \mathrm{C}\right)$ & 212 & 13.0 & 12.5 & 11.0 & 11.0 & 5.5 & 19.5 \\
\hline${ }^{*} \mathrm{pH}$ (standard units) & 212 & 7.6 & 7.5 & 7.4 & 7.4 & 6.8 & 8.0 \\
\hline "oxygen, dissolved (mg/L) & 197 & 6.0 & 4.5 & 1.0 & 2.3 & 0 & 8.9 \\
\hline *Alkalinity (mg/L as $\left.\mathrm{CaCO}_{3}\right)$ & 210 & 290 & 230 & 210 & 220 & 110 & 520 \\
\hline * Bicarbonate, endpoint titration ( $\mathrm{mg} / \mathrm{L}$ as $\left.\mathrm{HCO}_{3}\right)$ & 211 & 350 & 280 & 260 & 260 & 140 & 630 \\
\hline *Carbonate, endpoint titration (mg/L as $\mathrm{CO}_{3}$ ) & 211 & 0 & 0 & 0 & 0 & 0 & 0 \\
\hline * Carbon dioxide, dissolved (mg/L as $\left.\mathrm{CO}_{2}\right)$ & 211 & 34 & 22 & 18 & 21 & 3 & 160 \\
\hline *Hardness (mg/L as $\mathrm{CaCO}_{3}$ ) & 74 & 270 & 240 & 210 & 213 & 140 & 370 \\
\hline Calcium, dissolved (mg/L as Ca) & 74 & 73 & 68 & 58 & 60 & 39 & 100 \\
\hline Magnesium, dissolved ( $\mathrm{mg} / \mathrm{L}$ as $\mathrm{Mg}$ ) & 74 & 21 & 18 & 15 & 15 & 9.1 & 29 \\
\hline Sodium, dissolved (mg/L as $\mathrm{Na}$ ) & 74 & 24 & 17 & 14 & 15 & 9.7 & 39 \\
\hline Potassium, dissolved (mg/L as $\mathrm{K}$ ) & 170 & 13 & 5 & 3.2 & 5.4 & 1.5 & 50 \\
\hline Fluoride, dissolved ( $\mathrm{mg} / \mathrm{L}$ as $\mathrm{F}$ ) & 74 & .90 & .80 & .75 & .74 & .50 & .90 \\
\hline Chloride, dissolved ( $\mathrm{mg} / \mathrm{L}$ as $\mathrm{Cl}$ ) & 74 & 20 & 15 & 12 & 13 & 7.1 & 28 \\
\hline sulfate, dissolved (mg/L as $\mathrm{SO}_{4}$ ) & 93 & 38 & 35 & 30 & 30 & 12 & 49 \\
\hline silica, dissolved (mg/L as $\left.\mathrm{SiO}_{2}\right)$ & 74 & 25 & 23 & 21 & 21 & 13 & 28 \\
\hline DOC (carbon, organic dissolved, mg/L as $C$ ) & 147 & 2.60 & 2.0 & 1.1 & 1.4 & .3 & 4.4 \\
\hline Nitrogen, nitrite + nitrate, dissolved (mg/L as $N)$ & 200 & 2.49 & 1.50 & .55 & 1.18 & $<.10$ & 11.00 \\
\hline Nitrogen, ammonia, dissolved (mg/L as $N$ ) & 185 & 1.30 & .57 & .04 & .47 & $<.01$ & 7.30 \\
\hline Nitrogen, ammonia + organic, dissolved (mg/L as $\mathrm{N}$ ) & 150 & 2.67 & 1.10 & .50 & .99 & $<.10$ & 8.60 \\
\hline *Nitrogen, organic, dissolved (mg/L as $N$ ) & 99 & 1.20 & .53 & .37 & .54 & -.20 & 5.90 \\
\hline *Nitrogen, ammonia, dissolved (mg/L as $\mathrm{NH}_{4}$ ) & 143 & 2.20 & 1.20 & .10 & .78 & .01 & 9.40 \\
\hline Phosphorus, dissolved (mg/L as $P$ ) & 70 & .20 & .06 & .02 & .07 & $<.01$ & .62 \\
\hline Iron, dissolved $(\mu \mathrm{g} / \mathrm{L}$ as $\mathrm{Fe})$ & 179 & 2,200 & 620 & 30 & 650 & $<3$ & 7,000 \\
\hline Iron, total recoverable $(\mu \mathrm{g} / \mathrm{L}$ as $\mathrm{Fe})$ & 68 & 1,610 & 250 & 75 & 470 & $<10$ & 5,000 \\
\hline Manganese, dissolved $(\mu \mathrm{g} / \mathrm{L}$ as $\mathrm{Mn})$ & 179 & 3,100 & 2,200 & 180 & 1,090 & $<1$ & 5,100 \\
\hline 2 inc, dissolved $(\mu g / L$ as $2 n)$ & 151 & 130 & 90 & 51 & 66 & $<3$ & 320 \\
\hline
\end{tabular}

